\title{
Cost Efficient Provisioning of Mass Mobile Multimedia Services in Hybrid Cellular and Broadcasting Systems
}

DAVID GÓMEZ BARQUERO

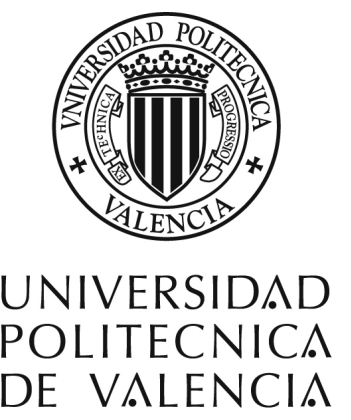

Doctoral Dissertation in Telecommunications Valencia, Spain 2009 



\section{Cost Efficient Provisioning of Mass Mobile Multimedia Services in Hybrid Cellular and Broadcasting Systems}

DAVID GÓMEZ BARQUERO

Doctoral Dissertation in Telecommunications

Valencia, May 2009 


\section{Supervisor}

Prof. Narcís Cardona Marcet

Communications Department

Universidad Politécnica de Valencia, Spain

\section{Reviewers}

Prof. Jens Zander

Department of Communication Systems

Kungliga Tekniska Högskolan, Sweden

Dr. Jarkko Paavola

Department of Information Technology

Turun Yliopisto, Finland

Prof. Pablo Angueira Buceta

Electronics and Telecommunication Department

Universidad del País Vasco - Euskal Herriko Unibertsitatea, Spain

\section{Opponents}

Prof. Thomas Kürner

Department of Mobile Radio Systems

Technische Universität Braunschweig, Germany

Prof. Gabriel Fernández i Ubiergo

Audiovisual Technologies Department

Universitat Ramon Llull, Spain

Prof. Vicente Baena Lecuyer

Department of Electronic Engineering

Universidad de Sevilla, Spain

Prof. Juan Carlos Guerri Cebollada

Communications Department

Universidad Politécnica de Valencia, Spain

Prof. Leandro Juan Llácer

Department of Information and Communications Technologies

Universidad Politécnica de Cartagena, Spain 


\section{Abstract}

One of the challenges that the wireless industry faces today is to provide mass multimedia services to mobile and portable devices at low cost, making them affordable to users and profitable to operators. The most representative service is mobile TV, which is expected to become a key application in future wireless networks. Nowadays cellular networks cannot support large scale consumption of such services, and newly deployed mobile broadcasting networks are very expensive to deploy due to the large investment in infrastructure required to provide acceptable coverage levels.

This dissertation addresses the problem of providing a cost efficient provisioning of mass mobile multimedia services with existing wireless infrastructure for digital broadcasting and cellular systems. State-of-the-art mobile broadcasting (DVB-H) and cellular (evolved 3G+ networks with HSDPA and MBMS) commercial technologies have been considered. Our approach is DVB-H centric. The main paradigm proposed to provide lower cost services is to avoid deployment a DVB-H network with high capacity and full area coverage from the beginning. Instead, it is proposed to perform an incremental DVB-H network deployment that follows the user demand. Within this context, the cellular network is essential to avoid over-dimensioning the broadcast capacity, and also in low populated areas until the deployment of a DVB-H transmitter or gap-filler becomes convenient.

The main technical solution proposed in this dissertation is to perform a multi-burst protection of the transmission in DVB-H using Raptor coding. The idea behind is to exploit the time diversity of the mobile channel to improve the robustness of the transmission, and thus the coverage level, at the expense of an increased network latency. Contrarily to most technical enhancements proposed in the literature, the introduction of this technique would be backwards-compatible with existing networks and terminals, since it could be introduced as a software update either at the link or the application layer.

On the other hand, this dissertation also investigates the potential efficiency improvement that can be achieved in a hybrid DVB-H/3G+ system when the cellular network is used to repair errors of the broadcast DVB-H transmissions. Raptor coding is also considered in this case, but at the application layer; since it enables an easy and efficient implementation of the repair mechanisms in such hybrid systems. 
In the dissertation we evaluate the potential gain that can be obtained with multi-burst forward error correction (FEC) in DVB-H compared to the conventional intra-burst FEC mechanism by means of simulations, lab and field measurements, and theoretical studies. Our results show that it is feasible to significantly increase the area coverage and the service quality in already deployed networks, as well as to reduce the investment in infrastructure required to deploy new networks. The gain is especially important for services where a large latency is not an issue, such as file download services. In these cases the area coverage can easily double, and even treble under certain conditions. The gain increases for large files, robust coding rates and for vehicular users due to their high mobility. Furthermore, for this kind of services without stringent time constraints it is possible to reduce the delivery cost by half combining a DVB-H network with a $3 \mathrm{G}+$ cellular network.

For DVB-H streaming services the gain due to multi-burst FEC is limited if low zapping times are desired. In this case it is necessary to combine the multi-burst coding with techniques aimed to reduce the zapping time from the perception of the users. However, it is possible to significantly increase the robustness of the transmission transmitting few services heavily protected. In particular it is possible to double the area coverage for vehicular users and up to $50 \%$ for pedestrian users. The proposed technique would also increase the service quality in already covered areas, removing practically all residual errors of the transmission.

This thesis received the second Rosina Ribalta award from Epson Foundation to the best projects of doctoral theses in the field of information and communications technologies presented in Spanish and Portuguese universities in 2009. 


\section{Resumen}

Uno de los retos a los que se enfrenta la industria de las comunicaciones móviles e inalámbricas es proporcionar servicios multimedia masivos a bajo coste, haciéndolos asequibles para los usuarios y rentables a los operadores. El servicio más representativo es el de TV móvil, el cual se espera que sea una aplicación clave en las futuras redes móviles. Actualmente las redes celulares no pueden soportar un consumo a gran escala de este tipo de servicios, y las nuevas redes de radiodifusión móvil son muy costosas de desplegar debido a la gran inversión en infraestructura de red necesaria para proporcionar niveles aceptables de cobertura.

Esta tesis doctoral aborda el problema de la provisión eficiente de servicios multimedia masivos a dispositivos móviles y portables utilizando la infraestructura de radiodifusión y celular existente. La tesis contempla las tecnologías comerciales de última generación para la radiodifusión móvil (DVB-H) y para las redes celulares (redes 3G+ con HSDPA y MBMS), aunque se centra principalmente en DVB-H. El principal paradigma propuesto para proporcionar servicios multimedia masivos a bajo coste es evitar el despliegue de una red DVB-H con alta capacidad y cobertura desde el inicio. En su lugar se propone realizar un despliegue progresivo de la infraestructura DVB-H siguiendo la demanda de los usuarios. Bajo este contexto, la red celular es fundamental para evitar sobre-dimensionar la red DVB-H en capacidad y también en áreas con una baja densidad de usuarios hasta que el despliegue de un transmisor o un repetidor DVB-H sea necesario.

Como principal solución tecnológica la tesis propone realizar una codificación multiburst en DVB-H utilizando códigos Raptor. El objetivo es explotar la diversidad temporal del canal móvil para aumentar la robustez de la señal y, por tanto, el nivel de cobertura, a costa de incrementar la latencia de la red. Contrariamente a la mayoría de mejoras tecnológicas propuestas en la literatura, la introducción de esta técnica sería compatible con las redes y terminales existentes en la actualidad, ya que se podría introducir como una actualización software en la capa de enlace o en la de aplicación.

Por otro lado, la tesis investiga la reducción de coste que se puede conseguir en un sistema híbrido DVB-H/3G+ cuando la red celular se utiliza para reparar errores de las transmisiones DVB-H. En este caso también se ha considerado una codificación Raptor, pero en la capa de aplicación, ya que posibilita una implementación sencilla y eficiente de los mecanismos de reparación en estos sistemas híbridos. 
En la tesis se evalúa la ganancia potencial que se puede obtener con una codificación multi-burst en DVB-H en comparación con la técnica de codificación convencional mediante simulaciones, medidas de laboratorio y campo, y estudios teóricos. Los resultados demuestran que es posible aumentar el nivel de cobertura y la calidad de servicio muy notablemente en redes ya desplegadas, así como desplegar nuevas redes con mucha menos infraestructura. La ganancia es especialmente importante para servicios en los que una latencia alta no es un problema, como, por ejemplo, en la transmisión de ficheros. En estos casos se podría fácilmente duplicar, y hasta triplicar en algunos casos, el área de cobertura. La ganancia aumenta para ficheros grandes, tasas de codificación robustas, y para usuarios en vehículos debido a su alta movilidad. Además, la tesis demuestra que para este tipo de servicios sin estrictas restricciones temporales la combinación de una red DVB-H con una red celular 3G+ puede reducir el coste de la transmisiones DVB-H hasta en un 50\%.

Para servicios de video-streaming la ganancia debida a una codificación multi-burst está limitada si se quieren proporcionar tiempos de zapping pequeños. En este caso es necesario combinar la codificación multi-burst con técnicas para reducir el tiempo de zapping de la percepción de los usuarios. Sin embargo, también es posible aumentar la robustez significativamente transmitiendo pocos servicios altamente protegidos. En concreto se podría duplicar el área de cobertura para recepción en vehículos, y hasta en un 50\% para recepción tipo peatón. La técnica propuesta también aumentaría la calidad de servicio en zonas ya cubiertas, eliminando en su práctica totalidad los errores residuales de la transmisión. 


\section{Resum}

Un dels reptes als que s'enfronta l'industria de les comunicacions mòbils i inalàmbriques és proporcionar serveis multimedia massius a baix cost, fent-los assequibles als usuaris i alhora rentables per als operadors. El servei mes representatiu és el de la TV mòbil, que s'espera sigui una aplicació clau en el futur de les comunicacions mòbils. Actualment les xarxes cel-lulars no poden suportar un consum a gran escala d'aquest tipus de serveis, i les noves xarxes de radiodifusió mòbil són cares de desplegar degut a la gran inversió en infraestructures de xarxa necessàries per a proporcionar nivells acceptables de cobertura.

Aquesta tesis doctoral aborda el problema del proveïment eficient de serveis multimedia massius a dispositius mòbils i portables, utilitzant la infraestructura de radiodifusió i cel-lular ja existent. La tesis contempla les tecnologies comercials d'última generació per a la radiodifusió mobil (DVB-H) i per a les xarxes cel-lulars (xarxes 3G+ amb HSDPA i MBMS), tot i que es centra principalment en DVB-H. El principal paradigma que s'ha proposat per a proporcionar serveis multimèdia massius a baix cost ès evitar el desplegament de una xarxa DVB-H d'alta capacitat i cobertura des del principi. En lloc d'això, es proposa realitzar un desplegament progressiu de la infraestructura DVB-H seguint el creixement de la demanda per part dels usuaris. En aquest context, la xarxa cel-lular és fonamental per a evitar sobre-dimensionar la capacitat de la xarxa DVB-H i també evitar costos en àrees en les que la densitat d'usuaris és baixa atraçant la instal-lació de transmissors i repetidors fins que siguin necessaris.

Com a principal solucio tecnològica aquesta tesis proposa realitzar una codificació multi-burst en DVB-H mitjançant codis Raptor. L'objectiu és explotar la diversitat temporal del canal mòbil per tal d'augmentar la robustesa del senyal i, per tant, el nivell de cobertura, a canvi d'incrementar la latència de la xarxa. Contràriament al que la majoria de millores tecnològiques propostes a la literatura, la introducció d'aquesta tècnica seria compatible amb les xarxes i terminals actuals, donat que es podria introduir com una actualització de software en la capa d'enllaç o en la de aplicació.

Per altra banda, la tesis investiga la reduccio de costos que es podem aconseguir en un sistema híbrid DVB-H/3G+ quan la xarxa cel-lular s'utilitza per a reparar errors en les transmissions DVB-H. En aquest cas també s'ha considerat una codificació Raptor, però en la capa d'aplicació, ja que possibilita una implementació senzilla i eficient dels mecanismes de reparació en aquests sistemes híbrids. 
En la tesis s'avalua el guany potencial que es pot obtenir amb una codificació multiburst a DVB-H en comparació amb la tècnica de codificació convencional mitjançant simulacions, mesures de laboratori i de camp, així com estudis teòrics. Els resultats demostren que és possible augmentar els nivells de cobertura i qualitat de servei molt notablement en xarxes ja desplegades, així com desplegar-ne d'altres amb força menys infraestructura. El guany és especialment rellevant per serveis en els que una latència alta no és un problema, com, per exemple, en la transmissió de fitxers. En aquests casos es podria fàcilment duplicar i fins i tot triplicar l'àrea de cobertura. El guany augmenta per fitxers grans, taxes de codificació robustes i per a usuaris en vehicles, degut a la seva alta mobilitat. A més la tesis demostra que, per aquest tipus de serveis sense restriccions temporals estrictes, la combinació de una xarxa DVB-H amb una xarxa cel-lular $3 \mathrm{G}+$ pot reduir el cost de les transmissions DVB-H fins a un $50 \%$.

Per els serveis de video-streaming el guany que s'obté d'una codificació multi-burst està limitat si aquests es volen proveir amb temps de zapping reduïts. En aquest cas és necessari combinar la codificació multi-burst amb tècniques per reduir el temps de zapping des del punt de vista dels usuaris. Tot $\mathrm{i}$ això, també és possible augmentar la robustesa molt significativament si es transmeten pocs serveis altament protegits. En particular, es podria duplicar l'àrea de cobertura per receptors en vehicles i fins a un 50\% en el cas de vianants. La tècnica proposta també augmentaria la qualitat de servei en zones ja cobertes, eliminant la pràctica totalitat dels errors residuals de la transmissió. 


\section{Acknowledgments}

To complete this dissertation has been a very intense and very rewarding experience. For this, I first would like to thank Prof. Narcís Cardona to offer me the possibility of doing my Ph.D. at his group. I also thank Prof. Cardona for the freedom I have been given in my research. I am also very grateful to Prof. Jens Zander (Royal Institute of Technology, Sweden), Dr. Jarkko Paavola (University of Turku, Finland), and Prof. Thomas Kürner (Technical University of Braunschweig, Germany) for inviting me to visit their research groups; which I found a very valuable experience both professionally and personally. I take the opportunity to thank all the people at Wireless@ KTH, the DTV group, and the IfN institute, which made me feel as one more of their teams. I am also grateful to Prof. Ulrich Reimers (Technical University of Braunschweig, Germany) and Dr. Thomas Stockhammer (Nomor Research, Germany) for inviting me to participate in the DVB standardization forum to present the results of my research.

The financial support during my studies should also be acknowledged. In particular I started my doctoral studies with an official Ph.D. scholarship from the Generalitat de Valencia, and I finish being supported by the Spanish Ministry of Industry, Tourism and Commerce through the project FURIA.

A special word goes to my three colleagues and very good friends: Aurelian Bria, Jussi Poikonen and Peter Unger. I could not have hoped for better people to work with! They have provided a great support to specific parts of my work, not only regarding technical issues, but also with respect to the research approach.

In addition to my colleagues, among the many people I have met I want mention my COST2100 friends; especially Virginia Corvino, Dejan Vukobratovic, Kristian Nyborn, Cedomir Stefanovic, and Silvia Zampese for sharing a very nice time during the meetings.

Back at Valencia, I want to thank my colleagues Josetxo, Paolo, Dani and Francisco for making office hours more bearable, and especially my thirteen Msc. students! Ariana, Paco, David, Ana, Jorge, Pablo, Gema, Jaime, Fernando, Pedro, Carlos, Valeria and Jordi. Without them life these years would have been much more boring.

Last but not least, I want to thank my family, my parents and my beloved brother and sister, for their infinite support throughout the years. They are simply the best family I could have. I dedicate this work to my grandparents in loving memory. 



\section{Contents}

$\begin{array}{ll}\text { Abstract } & \text { iii }\end{array}$

Acknowledgments $\quad$ ix

List of Abbreviations $\quad$ xiii

1 Introduction 1

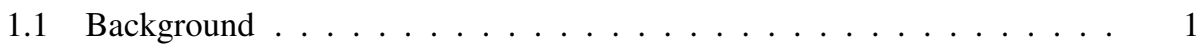

1.2 Hybrid Cellular and Broadcasting Systems . . . . . . . . . . . . . . . . 3

1.3 The Challenges of Mobile Multimedia Broadcasting . . . . . . . . . . . . 7

1.4 General Problem Description . . . . . . . . . . . . . . . . . . . 9

1.5 Dissertation Scope . . . . . . . . . . . . . . . . . . . . . . . . . . . . . . . . . .

1.6 Related Work . . . . . . . . . . . . . . . . . . . . . 13

1.7 Dissertation Outline and Contributions . . . . . . . . . . . . . . . 17

1.8 List of Publications . . . . . . . . . . . . . . . . . 22

2 Upper Layer Forward Error Correction in DVB-H 27

2.1 Mobile Multimedia Broadcasting Services and QoS in DVB-H . . . . . 28

2.2 Upper Layer Forward Error Correction . . . . . . . . . . . . . . . . . . 30

2.3 Forward Error Correction and Time Interleaving in DVB-H . . . . . . . . 42

2.4 AL-FEC in Hybrid Cellular and DVB-H Systems . . . . . . . . . . . 53

2.5 Conclusions . . . . . . . . . . . . . . . . . . 54

3 Multi-Burst Forward Error Correction in DVB-H 55

3.1 Field Measurements Results . . . . . . . . . . . . . . . . . . 58

3.2 Fading Margin Gain Simulation Results . . . . . . . . . . . . . . . 65

3.3 Conclusions . . . . . . . . . . . . . . . 73

4 DVB-H Deployment on Existing Wireless Infrastructure 75

4.1 Feasibility of DVB-H Infrastructure Deployment . . . . . . . . . . . . . 76

4.2 Scalability of DVB-H Infrastructure Deployment . . . . . . . . . . . 78

4.3 Use of Gap-Fillers in DVB-H Networks . . . . . . . . . . . . . . . . . . . . . 81

4.4 Network Planning Algorithm . . . . . . . . . . . . . . . . . . 83 
4.5 Coverage Estimation for Multi-Burst FEC Services . . . . . . . . . . . 86

4.6 Conclusions .......................... 90

5 Radio Resource Management in Hybrid Cellular and DVB-H Systems 93

5.1 Simulation Scenario . . . . . . . . . . . . . . . . . . 94

5.2 Filecasting in 3G Cellular Networks with MBMS and HSPDA . . . . . . 95

5.3 Filecasting in DVB-H Networks . . . . . . . . . . . . . . . . 103

5.4 Filecasting in Hybrid DVB-H/3G+ Systems . . . . . . . . . . . . . . 106

5.5 Conclusions . . . . . . . . . . . . . . . . . . 109

6 Conclusions and Future Work 111

6.1 Concluding Remarks . . . . . . . . . . . . . . . . . . . 111

6.2 Progressive DVB-H Network Deployment Discussion . . . . . . . . . . . 113

6.3 Future Research Issues . . . . . . . . . . . . . . . . . . . . . . . 114

A DVB-H Performance Evaluation Methods 117

A.1 Field Measurements . . . . . . . . . . . . . . . . . . . . . . . . 117

A.2 Dynamic Simulations . . . . . . . . . . . . . . . . . . 120

A.3 DVB-H Physical Layer Performance Model . . . . . . . . . . . . . . . . 130

A.4 Cellular System Models . . . . . . . . . . . . . . . . . . . . . . . . . . . . . . . . . . . . . . . . . .

A.5 Conclusions . . . . . . . . . . . . . . . . . 138

B Fading Margin Gain due to Multi-Slot FEC in TDM Broadcast Systems 139

B.1 Introduction . . . . . . . . . . . . . . . . . . . . . . . . 139

B.2 Problem Description . . . . . . . . . . . . . . . . . . 140

B.3 Theoretical Analysis with Uncorrelated Shadowing . . . . . . . . . . . . 143

B.4 Simulation Analysis with Correlated Shadowing . . . . . . . . . . . . . . 144

B.5 Validation with DVB-H Results . . . . . . . . . . . . . . . . . 146

B.6 Discussion File Download Services . . . . . . . . . . . . . . . . . . 147

B.7 Conclusions . . . . . . . . . . . . . . . . . . . . . 149

$\begin{array}{ll}\text { Bibliography } & 151\end{array}$ 


\section{List of Abbreviations}

1G, 2G, 3G Generations of Cellular Networks

$3 \mathrm{G}+$

Evolved 3G Cellular Networks with HSDPA and MBMS

3GPP Third Generation Partnership Program

AEBL Average Error Burst Length

ALC

Asynchronous Layered Coding

AL-FEC

ARPU

Application Layer - Forward Error Correction

$\mathrm{BCH}$

Average Revenue Per User

BER

Bose-Chaudhuri-Hochquenghem Codes

CAPEX

Bit Error Rate

$\mathrm{CDF}$

Capital Expenditures

CDP

Cumulative Distribution Function

CINR

Content Delivery Protocols

CNR

Carrier-To-Interference Plus Noise Ratio

COFDM

CR

Carrier-To-Noise Ratio

CRC

Coded Orthogonal Frequency Division Multiplexing

DSL

Code Rate

DVB

Cyclic Redundancy Check

DVB-H

Digital Subscriber Line

DVB-SH

Digital Video Broadcasting Project

DVB-T

Digital Video Broadcasting to Handhelds

DVB-T2

Digital Video Broadcasting Satellite Services To Handhelds

END

Digital Video Broadcasting Terrestrial

EP

Digital Video Broadcasting 2nd Generation Terrestrial

Equivalent Noise Degradation

ERP

Encoding Period

ESR

Effective Radiated Power

FEC

Erroneous Second Ratio

FER

Forward Error Correction

FFT

Frame Error Rate

FLUTE

Fast Fourier Transform

File Delivery Over Unidirectional Transport Protocol 


\begin{tabular}{|c|c|}
\hline GE & Gilbert-Elliott channel model \\
\hline GF & Galois Field \\
\hline GGSN & Gateway GPRS Support Node \\
\hline GI & Guard Interval \\
\hline GOP & Group of Pictures \\
\hline GPRS & General Packet Radio Service \\
\hline GPS & Global Positioning System \\
\hline GSE & Generic Stream Encapsulation \\
\hline GSM & Global System For Mobile Communications \\
\hline HNN & Hopfield Neural Networks \\
\hline HSDPA & High-Speed Downlink Packet Access \\
\hline HSPA & High-Speed Packet Access \\
\hline HSUPA & High-Speed Uplink Packet Access \\
\hline IETF & Internet Engineering Task Force \\
\hline iMB & Integrated Mobile Broadcast \\
\hline IP & Internet Protocol \\
\hline IP PER & IP Packet Error Ratio \\
\hline IPDC & IP Datacast \\
\hline IPTV & IP Television \\
\hline ISDB-T & Integrated Services Digital Broadcasting - Terrestrial \\
\hline LCT & Layered Coding Transport \\
\hline LDPC & Low-Density Parity Check codes \\
\hline LL-FEC & Link Layer Forward Error Correction \\
\hline LMS & Land Mobile Satellite \\
\hline LoS & Line of Sight \\
\hline LSE & Least-Squared Error \\
\hline LT & Luby Transform \\
\hline LTE & Long-Term Evolution \\
\hline MBMS & Multimedia Broadcast Multicast Services \\
\hline Media FLO & Multimedia Forward Link Only \\
\hline MER & Modulation Error Ratio \\
\hline MFER & MPE-FEC Frame Error Rate \\
\hline MPE & Multi Protocol Encapsulation \\
\hline MPE-FEC & Multi Protocol Encapsulation Fec \\
\hline MPEG-2 & Moving Picture Experts Group 2 \\
\hline MPE-iFEC & Multi Protocol Encapsulation Inter-Burst Forward Error Correction \\
\hline NLoS & Non-Line of Sight \\
\hline NMT & Nordic Mobile Telephone System \\
\hline NSGA-II & Non-Dominated Sorting Genetic Algorithm - Version II \\
\hline OFDM & Orthogonal Frequency Division Multiplex \\
\hline OPEX & Operational Expenditures \\
\hline
\end{tabular}




$\begin{array}{ll}\text { PDF } & \text { Probability Density Function } \\ \text { PER } & \text { Packet Error Rate } \\ \text { PI/PO } & \text { Pedestrian Indoor/Pedestrian Outdoor } \\ \text { p-t-m } & \text { Point-to-Multipoint } \\ \text { p-t-p } & \text { Point-to-Point } \\ \text { QAM } & \text { Quadrature Amplitude Modulation } \\ \text { QEF } & \text { Quasi-Error-Free } \\ \text { QoR } & \text { Quality of Restitution } \\ \text { QoS } & \text { Quality of Service } \\ \text { QPSK } & \text { Quadrature Phase-Shift Keying } \\ \text { RF } & \text { Radio Frequency } \\ \text { RRM } & \text { Radio Resource Management } \\ \text { RS } & \text { Reed-Solomon } \\ \text { RSSI } & \text { Received Signal Strength Indicator } \\ \text { RTP } & \text { Real-Time Transport Protocol } \\ \text { SER } & \text { Section Error Rate } \\ \text { SFN } & \text { Single Frequency Network } \\ \text { SINR } & \text { Signal-to-Interference Plus Noise Ratio } \\ \text { SNR } & \text { Signal-to-Noise Ratio } \\ \text { SRSE } & \text { Sliding Reed-Solomon Encoding } \\ \text { SUMO } & \text { Simulation For Urban Mobility } \\ \text { SVC } & \text { Scalable Video Coding } \\ \text { TCP } & \text { Transmission Control Protocol } \\ \text { TDM } & \text { Time Division Multiplexing } \\ \text { T-DMB } & \text { Terrestrial - Digital Multimedia Broadcasting } \\ \text { TS } & \text { Transport Stream } \\ \text { TTI } & \text { Transmission Time Interval } \\ \text { TU6 } & \text { Typical Urban 6-Tap Channel Model } \\ \text { TV } & \text { Television } \\ \text { UDP } & \text { User Datagram Protocol } \\ \text { UHF } & \text { Ultra High Frequency } \\ \text { UL-FEC } & \text { Upper Layer Forward Error Correction } \\ \text { VEBL } & \text { Variance Error Burst Length } \\ \text { VoD } & \text { Video-on-Demand } \\ \text { WCDMA } & \text { Wideband Code Division Multiplex Access } \\ \text { WLAN } & \text { Wireless Local Area Network } \\ & \end{array}$





\section{Chapter 1}

\section{Introduction}

\subsection{Background}

Mobile wireless communications are in constant evolution due to the continuously increasing requirements and expectations of both users and operators. Technology has gone through an amazing development during the last two decades, especially since the unexpected rapid adoption of voice and messaging services. The penetration rate of these services in the developed countries has reached a saturation level, and there are more mobile phones than fixed lines in most countries. Today, mobile data traffic is about to exceed voice traffic. Third-generation $(3 \mathrm{G})$ cellular networks are being deployed on a broad scale all over the world, and mobile operators have started to provide multimedia services ${ }^{1}$, such as video clips from sports events or live TV programs. According to several forecasts, it is expected that demand will explode in the next years, with more than half a billion customers will be subscribing to on their mobile devices by 2011 [1].

\section{From Voice Telephony Networks to Multimedia Delivery Networks}

The technological developments of mobile telephony have been classified using the concept of "generation". Generally speaking, a generation is associated to a specific transmission technology and a certain set of services. The first-generation (1G) is associated to the analogue technology (e.g., NMT). It was launched in the beginning of the 1980s. Its service offerings were exclusively targeted towards business users and the service penetration rate was low. A decade later, the second-generation (2G) introduced the digital technology (e.g., GSM); being able to provide not only voice services but also digital data services, such as messaging services. During the second half of the 1990s prices declined and mobile telephony was surprisingly soon adopted by most people in the developed countries.

\footnotetext{
${ }^{1}$ The global digitalization process of the communication and information technologies makes possible to combine multiple forms of information content (e.g., text, audio, and video) into a so-called multimedia service. Moreover, due to the intrinsic characteristics of digital data, the information can be easily produced, reproduced without any quality degradation, stored and manipulated.
} 
Both $1 \mathrm{G}$ and $2 \mathrm{G}$ networks were especially designed to support voice services with circuit-switched connections. This approach is efficient for applications with a relatively constant data rate such as voice, as a dedicated channel is reserved during all the time the connection is active. However, it is inadequate if the traffic generation rate varies significantly or when the traffic source is discontinuous. An intermediate generation, $2.5 \mathrm{G}$, is associated to the introduction of packet-based data services with packet-switched connections (at low data rates) in $2 \mathrm{G}$ networks (e.g., GPRS). Packet-switched connections provide higher resource efficiency compared to circuit-switched connections because different communications can share the same channel. Moreover, it allows users to virtually be connected without allocating dedicated network resources.

The $3 \mathrm{G}$ is associated to the provision of multimedia services. The European standard is known as UMTS, which is based on WCDMA (Wideband Code Division Multiplex Access) technology. Although the first release supports both circuit-switched and packetswitched connections, the trend is to evolve towards packet-switched all-IP (Internet Protocol) networks; meaning that all services will be carried on top of IP, even traditional voice services. $3 \mathrm{G}$ introduces a significant improved support for packet data services compared to previous generations of cellular systems, as well as higher data rates (in the order of few hundred $\mathrm{kb} / \mathrm{s}$ ) and system capacities. Still, the capabilities of the first release are considerably limited, from both a cost and a technical performance viewpoint. In order to offer a viable business model and to not overload the network capacity to the point of preventing subscribers from placing voice calls (which is the main function and value of the cellular networks), only short video clips with a low resolution could be offered [2]. To meet the increasing demands for high-speed data access, the 3G WCDMA standard was enhanced with HSPA (High-Speed Packet Access), for both downlink (HSDPA) and uplink (HSUPA) [3]. HSPA supports peak data rates up to several Mb/s, increasing considerably the network capacity.

Research and development towards the next generation of cellular networks has already started, also known as 3G long-term evolution (LTE) [4]. It is essentially driven by the desire to increase data rates at lower incremental costs, in order to support a larger variety of mobile multimedia services [5]. LTE will provide improved coverage and system capacity as well as increased data rates and reduced latency.

\section{Mass Mobile Multimedia Services}

In this dissertation we specifically focus on mass multimedia services, which are expected to generate a large amount of data traffic in future wireless networks [6]. Mass multimedia services are, by definition, purposed for many people. In general, it can be distinguished between the distribution of any popular content over a wide area, and the distribution of location-dependent information in highly populated areas. Representative examples include the delivery of live video streaming content (like sports competitions, concerts or news), and file download (multimedia clips, digital newspapers or software updates).

The most representative service today is mobile $T V$, which is commonly believed to bring great opportunities for society, just as the fixed TV and mobile telephony already have. Commercial trials reveal a strong consumer interest [7], [8]. 
The provision of digital television to mobile devices will bring new services for the consumers, which will in turn generate new business opportunities for all players of the media and telecommunications industries. Moreover, these services have the consent of the public administrations, as they clearly contribute to the Information Society development, enabling universal access to multimedia content everywhere at any time.

Current commercial mobile TV services offer traditional TV content on the move, but mobile TV can offer much more than that [9]. However, it will require significant content development and formatting to adapt offerings to the specifics of the mobile environment and market demand: small screens, time of the day, location, viewing duration, etc. Taking advantage of the intrinsic bidirectional communication capabilities of mobile phones, mobile TV services can introduce new forms of interactivity, customization and personalization, generating additional revenue streams and enhancing the value of the services to the consumers. New opportunities for advertising may be also opened up combining traditional TV advertisements with personalization features [1].

New revenue promising business opportunities may be also opened, offering not only live-to-air TV, but also video-on-demand ( $\mathrm{VoD})$. Mobile users typically prefer to access content on-demand, having the freedom to choose what to watch and when to watch it, rather than following a fixed schedule (the only exceptions are live events of high interest). Thanks to the continuously increasing computer processing and memory capabilities ${ }^{2}$, future mobile devices with massive storage capacities will enable "push and store" type of services, where the content is delivered (pushed) and stored on the terminals beforehand, and then played by the user, providing the illusion of instant transmission.

\subsection{Hybrid Cellular and Broadcasting Systems}

Traditionally, cellular systems have focused on the transmission of data intended for a single user using a dedicated point-to-point ( $\mathrm{p}-\mathrm{t}-\mathrm{p}$ ) radio bearer, not addressing the distribution of popular content to a large number of users simultaneously. Hence, $3 \mathrm{G}$ cellular systems (including HSPA) have been designed for unicast services delivered through p-t-p dedicated connections for each individual user, even if the same content should be delivered to many users.

Unicast systems can easily support a wide range of services, as each user may consume a different service. As each connection is intended for a single user, it is possible to optimize the transmission parameters for each user individually, and resources are also only employed when a user is actively consuming a service. The main drawback of unicast when delivering the same content to many users at the same time is its unfavorable scaling with the number of users. This limits the maximum number of users cellular systems can handle, since both radio and network resources are physically limited.

\footnotetext{
${ }^{2}$ Moore's Law is the empirical observation made in 1965 that the number of transistors on an integrated circuit for minimum component cost doubles every 24 months. It is attributed to Gordon E. Moore, co-founder of Intel.
} 
Multicast and broadcast ${ }^{3}$ are more appropriate transport technologies to cope with high numbers of users simultaneously consuming the same content when compared to unicast. Multicast/broadcast wireless transmissions employ a common point-to-multipoint ( $\mathrm{p}-\mathrm{t}-\mathrm{m}$ ) radio bearer for all users, which allows delivering the same content to an unlimited number of users within the covered area [10]. On the other hand, they cannot support a very high number of personalized services. User specific adaptation of the transmission parameters is generally not possible, and the transmission has to be designed to serve the worst-case user. Furthermore, retransmissions of lost packets on-demand are generally not feasible to avoid congestion (the so-called feedback implosion problem in multicast delivery). Error correction is achieved with forward error correction (FEC) mechanisms transmitting additional repair data such that receivers can reconstruct the original information even if some transmission error occurs. The error correction is "forward" in the sense that there is no feedback (return channel) from the receiver to the transmitter.

In order to efficiently transmit the same content to several users simultaneously, the $3 \mathrm{G}$ standard has been enhanced with MBMS (Multimedia Broadcast Multicast Services) ${ }^{4}$ [11], introducing new p-t-m radio bearers and multicast support in the core network. MBMS will lead to a better utilization of the existing radio resources provided by $3 \mathrm{G}$, enabling the provision of new multimedia multicast services. Its potential to efficiently provide mass multimedia services is rather low, due to the facts that it offers a limited capacity and it shares the same cell resources with voice, messaging and data services ${ }^{5}$.

The highest potential is currently presented by new mobile broadcast technologies specifically designed for mobile services [12]. Only these technologies can support large scale consumption of mass multimedia services such as mobile TV. The European standard for terrestrial mobile broadcasting is known as $D V B-H$ (Digital Video Broadcast Handheld) [13], [14]. After a slow start, and with a clear endorsement of the European Commission [15], DVB-H networks are being deployed across Europe. Italy was the first country to launch commercial services in summer 2006. Nowadays DVB-H is also on air in the main urban areas of Finland, Switzerland, Austria, the Netherlands, Hungary and Albania; and it is expected that more European countries will follow.

\footnotetext{
${ }^{3}$ Multicasting and broadcasting describe different, although closely related, scenarios. Whereas broadcast transmissions are intended for all users in the service area, multicast transmissions are addressed to a specific group of users (usually called the multicast group). Thus, broadcast can be considered as a particular case of multicast.

${ }^{4}$ MBMS is split into the MBMS bearer service and the MBMS user service, in such a way that it is possible to integrate $\mathrm{p}-\mathrm{t}-\mathrm{p}$ and $\mathrm{p}-\mathrm{t}-\mathrm{m}$ radio bearers in a transparent way to the MBMS service layer. Thus, it is possible to delivery an MBMS service with p-t-p transmissions. For the sake of clarity, we associate MBMS only to p-t-m transmissions and HSDPA to p-t-p transmissions.

${ }^{5}$ MBMS can be deployed in the unpaired time-division duplex (TDD) 3G spectrum, which is currently unused, without impacting current $3 \mathrm{G}$ voice and data services (which employ the paired frequency-division duplex, FDD, $3 \mathrm{G}$ spectrum). Since its introduction in $3 \mathrm{G}$ release 6, MBMS is under continuous enhancement. Release 8, also known as iMB (integrated Mobile Broadcast), will provide improved coverage and capacity performance, including single frequency network operation mode [16]. The specifications are expected to be completed during 2009.
} 
Although alternative technologies exist, such as T-DMB (Terrestrial - Digital Multimedia Broadcasting) in South-Korea [17], ISDB-T (Integrated Services Digital Broadcasting - Terrestrial) in Japan [18], and Media FLO (Multimedia Forward Link Only) in USA [19]; DVB-H seems today the most relevant mobile broadcasting technology worldwide, as it is also being deployed in South-East Asia and Africa. A key to the success of DVB-H is that it is introduced almost exclusively as a link layer on top of the European standard for digital terrestrial TV (DTT) DVB-T (Digital Video Broadcast - Terrestrial), which is the most widely adopted system in the world [20]. This way it is possible to share the same network infrastructure (e.g., transmitters, antennas, multiplexers).

\section{IP Datacast over DVB-H Systems}

Traditionally, terrestrial broadcasting networks have focused on delivering TV programs to large audiences covering very large areas. Existing infrastructure characterized by very tall towers and large transmission power, and the significant chunk of allocated spectrum in the UHF band represent extremely valuable resources. Moreover, DVB-T/H make use of OFDM (Orthogonal Frequency Division Multiplex) technology, which is particularly well-suited to the needs of the terrestrial broadcasting radio channel because it enables single frequency networks (SFNs) [21]. In SFNs all transmitters operate at the very same frequency and receivers combine signals coming from several transmitters. Transmitters are synchronized in both time and frequency using a GPS reference signal. SFN achieve a huge spectrum efficiency compared to analogue TV systems, where the same frequency channel cannot be re-used over a long distance because of the co-channel interference.

DVB-T was primarily designed for fixed rooftop reception, and it presents an important degradation in mobile channels [14]. Moreover, it is not practical for handheld devices small, lightweight, portable, single built-in antenna reception and battery-powered. The main features introduced with DVB- $\mathrm{H}^{6}$ are:

- Discontinuous transmission technique known as time-slicing, which reduces the power consumption of terminals.

- Optional intra-burst FEC mechanism at the link layer called MPE-FEC, which ensures more robust transmissions under mobility and impulsive interference conditions.

- The use of IP. Multi protocol encapsulation (MPE) is the adaptation protocol used to transport IP streams over MPEG-2 DVB-T transport streams.

With time-slicing data is periodically transmitted in bursts of maximum size $2 \mathrm{Mb}$, see Figure 1.1. Each burst contains information of the time difference to the next burst of the same service. Terminals synchronize to the bursts of the desired service and switch their receivers front-end off when bursts of other services are being transmitted.

\footnotetext{
${ }^{6}$ DVB-H also provides higher flexibility in the design of SFN (i.e., more options of cell sizes and support for high velocity terminals).
} 

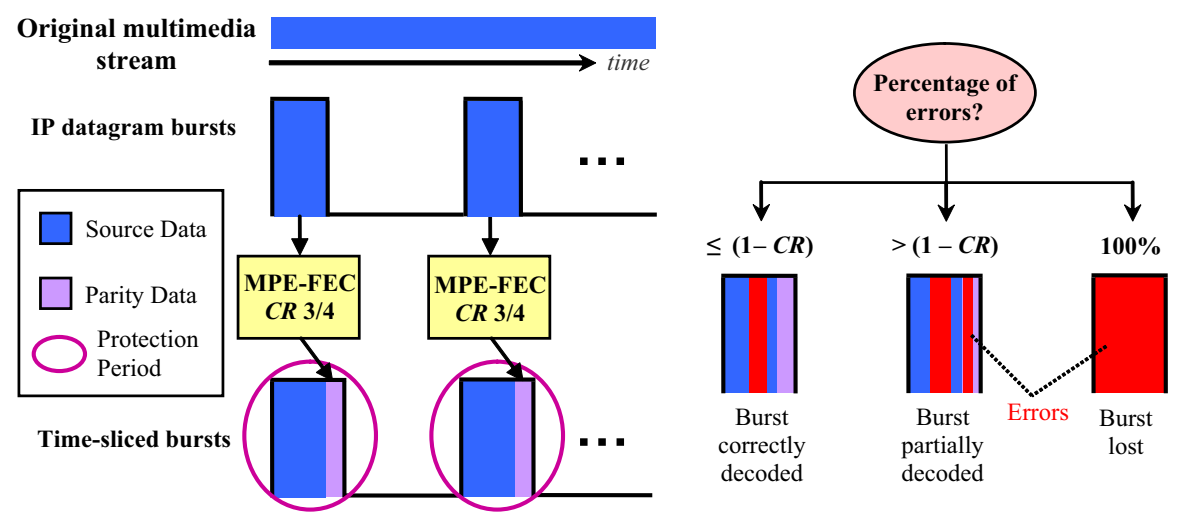

Figure 1.1: Time-slicing and MPE-FEC concept in DVB-H.

When MPE-FEC ${ }^{7}$ is employed, each IP datagram burst is encoded with a Reed-Solomon (RS) code at the link layer to generate parity data which is transmitted with the source IP data to compensate for potential transmission errors. The maximum percentage of errors per burst that can be corrected is equal to the rate of parity data transmitted. For example, a code rate $3 / 4$ can cope with up to $25 \%$ errors. Therefore, MPE-FEC can cope with errors that represent a fraction of the burst, but it cannot recover from complete lost bursts.

DVB-H is a transmission standard with an IP interface that specifies the physical and link layers, but the end-to-end system is known as IP datacast (IPDC) [22]. The set of IPDC specifications ${ }^{8}$ contribute with the higher layer protocols to build a complete endto-end system, and it specifies, among others, content delivery protocols for streaming and file download services, the electronic service guide for service discovery, and mechanisms for service purchase and protection. Although originally designed for use with DVB-H, IPDC has been designed to be bearer independent, being the IP layer the bearer abstraction layer. The IPDC specifications also describe all components required for complementing a mobile broadcasting network with a bi-directional interactivity path offered by the cellular systems.

IPDC is thus an interworking platform that enables the convergence of services from broadcast and cellular domains, combining the strengths of DVB-H to efficiently deliver very popular content with the personalization, interaction and billing features of cellular networks. In this sense, the DVB-H network is a complement of the cellular system. It should be pointed out that there is no convergence in networks, since both DVB-H and cellular networks are used separately and independently [23]. Convergence is realized in services, platforms and multi-mode terminals which support both radio access technologies [24].

\footnotetext{
${ }^{7}$ MPE-FEC operation mode is described in detail in Chapter 2.

${ }^{8}$ OMA BCAST is another set of specifications similar than IPDC but with a different emphasis. In particular, OMA BCAST integrates a DVB-H network into a mobile operator infrastructure. Work is on-going towards harmonization both sets of specifications.
} 


\subsection{The Challenges of Mobile Multimedia Broadcasting}

\section{The Cost of Providing Mobile Multimedia Broadcasting Services}

The success of mobile telephony was enabled by the low cost provision of both voice and messaging services. These services only require a low average and peak data rate per session. The problem with multimedia services is that the value of the bits is not perceived in the same way as the voice calls are, and user satisfaction does not scale with the amount of bits received. Therefore, since the aim is to provide several orders of magnitude higher data rates, the cost per transmitted bit has to radically drop [25]. Unfortunately, to support high data rates with wide area coverage at low cost still requires substantial technological advances. Traditional network planning for digital broadcast systems is based on a worstcase analysis, which results in over-dimensioning the network infrastructure. Such worstcase reception conditions must be supported in order to ensure signal reception in very adverse conditions and maximize the potential number of subscribers that can receive the content.

The most important bottleneck is the cost of the wireless infrastructure, which is proportional to the data rate provided to the user terminal in a system with wide coverage [26]. Wireless communications systems are confined to the rate/range trade-off provided by the "Shannon bound" of communication theory". This is due to the fact that a minimum amount of energy is required to reliably transfer every bit of information. The higher the data rate, the less energy is available for every bit at the transmitter, and thus the feasible communication distance is smaller ${ }^{10}$. As a consequence, providing broadband access over a wide area inevitably requires a large number of access points (base stations), which implies larger investments. It has been shown that the number of access points required grows about linearly with the data rate provided [26], meaning that the cost per transmitted bit is virtually constant. Hence, higher user data rates directly translate into higher cost.

\section{Mobile Broadcasting Business Case}

The expenses associated with building, maintaining, and servicing a mobile broadcasting network can be high, but that does not mean that a viable business model is not possible [27]. Broadcast is economically advantageous because the infrastructure cost is amortized over the user base. As the user base grows, the cost per subscriber falls. Note that this is unlike unicast cellular architectures, where the cost per supported user is fixed. Hence, everything comes down to consumer demand and population density ${ }^{11}$.

\footnotetext{
${ }^{9}$ According to the Shannon bound, data rates $R$ increase linearly with spectrum bandwidth $W$, but only logarithmically with the signal to interference plus noise ratio SINR. For an additive white Gaussian channel $R=W \cdot \log _{2}(1+S I N R)$.

${ }^{10}$ For a given transmit power and bandwidth, carrier frequency and radio access technology. The carrier frequency also strongly affects the area coverage that can be served with an access point for a given bandwidth and data rate, as the propagation path loss increases as a function of the carrier frequency.

${ }^{11}$ From this simple discussion it should be clear that if more than one network is built, the profit potential would shrink proportionally.
} 
Reaching the break-even point to recover the costs for infrastructure depends on the user population and the average revenue per user (ARPU). The business will be thus more likely to succeed in populated areas and where there is a high consumer demand ${ }^{12}$. This fact may prevent achieving nation-wide coverage, limiting the deployment to urban areas ${ }^{13}$.

The main business aspects when introducing a new mobile broadcast technology such as DVB-H are the necessary capital expenditures (CAPEX) and operating expenditures (OPEX) together with the consumer demand. Basically, the high-level questions are:

- How much investment is needed to deploy a mobile broadcast network, and what is the annual operational cost?

- To what extend are consumers willing to pay for mobile broadcasting services?

A complete answer to these questions requires considering many aspects related to technology, business and regulations, which exceed the size and scope of this dissertation. But it is clear that nowadays paying for mobile TV content is not a mass impulse. Although early trials were encouraging to operators and willingness to pay was high [7], [8], recent studies and results of the first commercial DVB-H networks are not so encouraging. Today, it is commonly accepted that mass-market demand for mobile multimedia entertainment is conditioned to the provision of these services at low cost.

The demand for mobile broadcasting basically depends on the availability of content, the subscription fee and the price of handsets. By offering the right mix of incentives to consumers it may be possible to drive the business forward, but TV broadcasters, broadcast and cellular network operators, and handset manufacturers must cooperate and lower their prices to ensure that the demand can be met and maximize the technology adoption rate [29]. This can be done by offering more free-to-air content (which does not preclude from offering premium content), subsidizing handsets and reducing the subscription price. Encouraging a quick formation of a large customer base increases the long-term profit potential for everyone, and it even creates a potentially lucrative platform for advertisers. Moreover, powerful imitation effects may also increase the demand, while consumers see others using the technology and desire it for themselves.

\section{Business Models and Regulatory Barriers}

Even being very promising from the technical side, the successful deployment of new hybrid cellular and broadcasting systems is also conditioned by several business and regulatory related aspects.

\footnotetext{
12 This explains why mobile TV services are a success in South-East Asian countries, as they are both highly populated and technology driven. In Europe, Italy was the most successful 3G mobile TV market prior the introduction of DVB-H, even though there were only a few channels and the quality was best effort with a price that was quite high.

13 DVB-H can be complemented with the new standard DVB-SH (Digital Video Broadcasting - Satellite Services to Handheld Devices) [28], designed for the provision of mobile broadcasting services through hybrid satellite-terrestrial networks. This hybrid architecture allows nation-wide coverage in Europe in a fast and efficient way thanks to the satellite transmission, whereas the terrestrial component ensures coverage in urban areas where direct reception of the satellite signal is not possible.
} 
The problem is that broadcast and cellular networks belong to different industries, each of them developed in a different business environment under very different value chains and revenue models.

Mobile broadcasting will give rise to several business models with various possible roles [30]. The balance between TV broadcasters, cellular network operators and broadcast network operators will depend on the local regulatory regime. A good description of the country-specific implementations can be found in [31]. Assuming that no player will be able to control the complete value chain in most countries, broadcasters and cellular network operators may need to work together, but need a suitable business model in an extremely complex business environment, each playing to their strengths.

A collaborative model can be achieved with the presence of a wholesaler of the mobile broadcasting service acting as datacast operator. This model is considered to provide the best chances to establish a successful business model for mobile broadcasting, and leaves potential retailers negotiate a sustainable business case enabled by cooperation [32]. The key objective is to have one neutral service provider (wholesaler), controlling a single mobile broadcasting network and sharing the scarce spectrum resource with several cellular network operators and content providers. The wholesaler can be a consortium of cellular operators, a broadcast network operator (due to its neutral position in the market and technical competence), or any third party. The service provider is responsible for the technical service provision, and acts as a facilitator for the cellular operators in the aggregation of content and the usage of broadcast transmission capacity. This model enables fully interactive services and a common bill for users, since the cellular operators bill their customers for the mobile broadcasting service. Moreover, it also overcomes the inefficient usage of spectrum resources because cellular operators share a common service package, being also possible to differentiate their service offerings to some extent.

\subsection{General Problem Description}

\section{DVB-H Deployment on Existing Wireless Infrastructure}

One of the major concerns about the roll-out of DVB-H is the network infrastructure cost, which could lead to a price for service that is unaffordable for most users. This concern is justified if the dimensioning of the network infrastructure is performed to support realtime streaming services, high capacity ${ }^{14}$ and nearly full area coverage, as in traditional broadcasting. DVB-H terminals suffer from much more severe propagation conditions than DVB-T. Not only in terms of increased path loss ${ }^{15}$, but also because of signal fading effects as the result of multipath propagation and shadowing due to the mobility of the users $^{16}$.

\footnotetext{
14 The envisaged commercial requirement for DVB-H when it was developed was $10 \mathrm{Mb} / \mathrm{s}$ [13].

15 The link budget difference between fixed rooftop reception and pedestrian outdoor reception due to antenna gain and height loss is over $30 \mathrm{~dB}$ [33]. Pedestrian indoor and vehicular (in-car) reception present additional losses due to building and vehicle penetration. This issue is further elaborated in Chapter 4.

16 The impairments of the mobile wireless channel are described in more detail in Appendix A. We will discuss DVB-H transmission errors more thoroughly in Chapter 3.
} 
The additional protection mechanism MPE-FEC basically copes with multipath (fast) fading in covered areas where static reception is possible. With MPE-FEC it is possible to recover from bursts partially received, and it is particularly useful for vehicular users when they experience strong fast fading impairments [14]. But MPE-FEC cannot recover from completely erroneous bursts, which may occur when passing through outage areas but also in covered areas due to temporary shadowing. Lost bursts have a major impact in the service coverage because they are perceived by the users as a coverage discontinuity. Hence, very high location probabilities are required ${ }^{17}$.

As most digital TV sites cannot increase their transmission powers ${ }^{18}$, a considerably large number of new sites is required for the installation of additional DVB-H transmitters or repeaters (gap-fillers), complementing the existing broadcasting towers and forming very dense SFNs [36]. This penalty is particularly evident for high area coverage targets (over $90 \%$ of service area locations), and implies very large investments in infrastructure [37]. The reason is that it is especially costly to guarantee coverage to the last few per cent of the worst-served locations.

\section{Problem Definition}

The delivery of mass multimedia content to mobile and portable devices is a very challenging task, as both massive scalability and reliable transfer are required. Moreover, nowadays it is acknowledged that a thriving mass mobile multimedia market will not develop unless services are provided at low cost, making them affordable to users and profitable to operators.

The high-level problem of this dissertation is: how hybrid cellular and broadcasting systems should be deployed to provide mass mobile multimedia services in a cost effective manner.

The focus is on hybrid IP datacast DVB-H systems with an evolved 3G+ cellular network with HSDPA and MBMS capabilities.

\subsection{Dissertation Scope}

\section{Problem Approach}

Based on the discussion presented above, in this dissertation a framework for a cost efficient provisioning of mass mobile multimedia services reusing the existing infrastructure for digital broadcasting and cellular systems is proposed based on a progressive deployment of the DVB-H infrastructure [38]. A progressive deployment is of special importance in the initial phases of the network rollout of new wireless communication systems, when the user population is small and services are still under development [39].

\footnotetext{
${ }^{17}$ In DVB-H the service availability at a certain location can be defined as the probability of correctly receiving a burst. One location is considered as good covered if the average burst error rate is below 5\% [34]. High location probabilities are necessary due to the very rapid transition from near perfect reception to no reception at all characteristic of digital systems, in opposition to the gradual failure typical of analogue systems.

18 To avoid interference and due to electromagnetic exposure limits of the current international regulation [35].
} 
Since investment in new infrastructure is very costly, it is expected that existing broadcasting towers will be initially used to provide a basic DVB-H coverage, and additional transmitters or gap-fillers will be progressively deployed in critical areas (e.g., where indoor or vehicular reception is required), co-sited with cellular base stations if possible [40]. However, we argue that avoiding full DVB-H area coverage and very high broadcast capacity from the beginning is probably the key to providing affordable mobile broadcasting services.

Multi-mode cellular/DVB-H terminals enable new ways of delivering multimedia services by jointly using both radio access networks, as users do not care about which radio accesses their phone can handle and which one they are actually using. User perceived reliability and coverage can be significantly enhanced by the redundancy offered by both networks, achieving an efficient usage of the radio resources ${ }^{19}$. Especially, evolved $3 \mathrm{G}+$ cellular networks present a very good potential to complement DVB-H networks due to the recent enhancements of the $3 \mathrm{G}$ WCDMA standard that allow high-speed p-t-p connections with HSDPA and p-t-m transmissions with MBMS [42].

The combination of a DVB-H network with a $3 \mathrm{G}+$ network into a hybrid cellular and broadcasting system allows for an incremental deployment of the DVB-H infrastructure, following the user demand and adding new sites only where required and when needed [43]. In the initial phases of the DVB-H network roll-out the cellular network should be used not only to avoid broadcasting services that few people watch, but also to avoid deploying new DVB-H sites in low populated areas until it is economically justified. This approach leverages existing wireless infrastructure, and reduces the investment risks in the technology. Moreover, it also provides a faster time-to-market. Typically, large scale infrastructure deployment might take years, whereas new user equipments and servers appear on the market in months.

\section{Proposed Technical Solutions}

In this dissertation, two particular technical solutions are treated and elaborated for a cost efficient provision of mass mobile multimedia services in hybrid evolved $3 \mathrm{G}+$ cellular and broadcasting DVB-H systems:

- Multi-burst forward error correction in DVB-H.

- Hybrid broadcast-cellular delivery in hybrid DVB-H/3G+ systems.

Both solutions aim to avoid over-dimensioning the DVB-H network in coverage, alleviating the infrastructure requirement to start providing DVB-H services over a target service $\operatorname{area}^{20}$. In our framework the DVB-H network will thus provide, to some extent, only partial coverage over the service area, and mobile terminals will experience temporary lack of coverage.

\footnotetext{
19 Higher spectrum efficiencies can be achieved by sharing the spectrum resources of both networks in a dynamic manner [41].

${ }^{20}$ We use the term service area to refer to the target area where mass mobile multimedia services are to be provided. This should be distinguished from the DVB-H covered area, the area where the DVB-H radio signal with sufficient quality is available.
} 
The first solution exploits the discontinuous transmission pattern of DVB-H together with the mobility of the users to recover from temporary errors at the expense of a decreased broadcast capacity. The second solution exploits the existence of multi-mode terminals, with both cellular and broadcasting radio access technologies, to deliver the content more efficiently at the expense of a decreased cellular capacity.

FEC is a key element in wireless broadcast communication systems, since any coding efficiency improvement has a positive business impact. FEC enhancements bring link margin gains that can be used to reduce the required infrastructure cost to provide the service over a given area, or to increase the coverage area with the same infrastructure bringing the broadcast signal to more users.

DVB-H inherits the physical layer FEC of the DVB-T standard, which corrects bit errors within MPEG-2 transport stream (TS) packets. To increase the robustness of the DVB-H transmissions while keeping compatibility with DVB-T, MPE-FEC was introduced at the link layer to recover from bursts partially received. However, MPE-FEC cannot recover from completely erroneous bursts. In order to recover from these errors, a FEC scheme spanning multiple bursts is required [44]. The basic idea of performing a multi-burst protection of the transmission is to exploit the time diversity of the mobile channel, increasing the time interleaving of the information. With multi-burst FEC the transmission robustness can be enhanced not only as a function of the capacity devoted for error repair but also as a function of the number of bursts jointly encoded. The drawbacks are that the network latency increases and the terminal memory requirement are larger, because terminals must wait and store all bursts jointly encoded before decoding.

On the other hand, in a hybrid cellular and DVB-H system the cellular network can be also used for error repair of the broadcast transmissions. This hybrid broadcast-cellular delivery is potentially more efficient than using a single DVB-H transmission [45]. Intuitively, in a realistic scenario there will always be some users that experience significantly worse DVB-H reception conditions than the majority (due to fading, noise, interference), such that it may be more efficient to serve them through the cellular network. Although the capability of the cellular network to deliver repair data depends on the distance between users and base stations, and other external factors as the network load, the combination of DVB-H with MBMS and HSDPA may reduce the delivery cost significantly.

\section{Research Problems}

The first research problem addressed in this dissertation is to devise multi-burst FEC schemes for $\mathrm{DVB}-\mathrm{H}$. Although DVB-H is nowadays considered a mature technology, recent advances in FEC mechanisms could lead to more affordable mobile broadcasting services. The main issue, however, is that new FEC solutions are usually not backwardscompatible with existing network infrastructure and terminals because dedicated hardware is required ${ }^{21}$.

\footnotetext{
${ }^{21}$ This is also the main problem for alternative solutions to improve the coverage such as advanced antenna systems [47].
} 
To increase the robustness of the DVB-H transmission maintaining compatibility with legacy equipment, additional FEC schemes have to be implemented at the link and application layers using the MPE-FEC memory available at the terminals (the memory requirement is $2 \mathrm{Mb}$ per stream decoded) [46].

The second research problem addressed is to evaluate the potential link margin gain that can be achieved with multi-burst FEC compared to the conventional MPE-FEC intraburst mechanism. Conventional DVB-H network planning is based on a static approach that targets to guarantee a certain coverage level (i.e., percentage of locations where average signal strength exceeds a given value with a high probability target). However, with multi-burst FEC the service availability cannot be considered a static measure, since the performance depends on the mobility of the users. Therefore, a revision of the traditional coverage estimation and network planning methods is needed in order to assess the coverage of multi-burst FEC services in real scenarios. In this dissertation we develop such methodology.

The third research problem consists in evaluating the operational cost savings that can be achieved using HSDPA and MBMS to repair DVB-H transmission errors. From a radio resource management perspective, a hybrid DVB-H/3G+ system combines the best of cellular and broadcast existing technologies, and enables three ways of transmitting data: unicast with HSDPA, multicast with MBMS, and broadcast with DVB-H. In such system it becomes crucial a proper joint management of the available radio resources in order to exploit their potential benefits. In the dissertation we will first analyze multicast delivery in $3 \mathrm{G}+$ networks with HSDPA and MBMS, and radio resource management issues in DVB-H networks before analyzing a hybrid DVB-H/3G+ system. We will not address the decision on whether to transmit the service via DVB-H or the cellular network by applying a load balancing algorithm. We will consider that the service is popular enough such that it should be transmitted through DVB-H.

\subsection{Related Work}

Before we introduce the contributions of the dissertation, an overview of related prior work is given next.

\section{Forward Error Correction in DVB-H}

One effective way to improve the DVB-H performance is to replace the concatenated ReedSolomon (RS) and convolutional DVB-T code at the physical layer with a more powerful code, such as a turbo-code [48] or an LDPC code [49]. Although link margin gains of few decibels are feasible, these solutions are not backwards-compatible with legacy equipment because they imply modifications at the physical layer.

Another topic that has brought attention in the literature is the optimization of the MPE-FEC decoding process [50], [51]. Basically, the goal is to minimize the amount of data needed such that the MPE-FEC decoding is successful. 
In DVB-H each burst consists of an integer number of MPE sections. Each IP packet is encapsulated into a section. At the receivers typically each section is considered either completely received or completely lost based on a cyclic redundancy check (CRC) field. Hence, one single erroneous TS packet can yield up to two erroneous MPE sections (TS packets have a fixed size of 188 bytes, whereas typical sections sizes range between 0.5 and $1.5 \mathrm{kB}$ ). Therefore, there is a potential gain working with MPEG-2 TS packets instead of MPE sections, and using the CRC field of MPE sections for additional protection (this would require changes to the standard). The potential gain is, however, not significant, and it does not justify the increased complexity at the receivers. Results from field trials show that TS packet error patterns are heavily correlated, such that several consecutive packets are lost, and the loss rates at the physical and link layers are practically the same [52]. These techniques also not solve the problem of recovering completely erroneous bursts.

One alternative to recover from these errors is to split the burst in smaller parts [53]. This approach is somehow equivalent to perform a multi-burst protection of the transmission, but with very small bursts and with a limited number of bursts jointly encoded. The time diversity is limited to one cycle time value, which is usually in the order of one to few seconds. Hence, the gain is very small.

The most relevant FEC advance in DVB-H for this dissertation was the adoption of Raptor coding at the application layer for file download services, commonly referred to as application layer FEC (AL-FEC) [54]. Raptor codes are a computationally efficient implementation of fountain codes that achieve close to ideal performance [55]. They were previously adopted in 3G MBMS for both streaming and file download services [56]. The main benefits of Raptor coding are that they can encode large amounts of data and that they can be implemented in software without the need of dedicated hardware. Therefore, they can be used in DVB-H to provide protection across several bursts, while being backwardscompatible with existing networks and terminals as they can be introduced as a software update. In this dissertation we will investigate the use of Raptor codes for DVB-H streaming services.

Regarding performance evaluation results, the main literature is the standardization work for the IPDC content delivery protocols [57], [58]. Simulation results are provided to show that it is possible to increase the area coverage compared to MPE-FEC because it is possible to cope with temporary shadowing. Results also show that although MPE-FEC and AL-FEC can be applied at the same time, it does not bring any benefit compared to AL-FEC alone. However, although the significant gain that can be achieved is highlighted, guidelines for configuration of Raptor coding are merely illustrative. In [59] it is shown with rather simple models that the gain due to shadowing reduces when the receiver is moving slowly.

\section{DVB-H Network Planning and Deployment}

Generally speaking, the objective of network planning for digital terrestrial broadcast networks is to provide sufficient signal quality over the service area with minimal network cost while keeping the potential interference under a specified level [60]. 
Optimizing the network configuration is crucial because it can significantly influence the base cost of providing a service. Moreover, in dense SFNs proper network planning is required to take full advantage of the diversity gain, also called network gain. In a SFN many receiving locations are served by more than one site, introducing redundancy in the transmitted signal and improving the coverage. DVB-H network planning is, to some extent, similar to DVB-T network planning; but it requires a considerable larger number of sites and the use of low power transmitters or gap-fillers. Efficient planning of wireless networks is a very well studied topic in the literature, since the optimization of the sites' location, as well as the different system parameters results in considerable network cost savings. Nowadays, it is usually performed with multi-objective optimization techniques, since they can solve problems with opposite optimization criteria, like minimizing the network cost and maximizing the area coverage.

There are two main strategies to solve this problem. The first one is based on the optimization of a single aggregated function by means of mono-objective optimization techniques (e.g., simulated annealing) as in [61]. The use of this strategy implies the combination of several objectives in a single function, which can lead to benefit or to penalize some of them. For example, the optimization problem can be formulated as to minimize the network cost subject to guaranteeing a certain area coverage target. On the other hand, the second strategy consists in obtaining a set of optimum solutions, which can be considered equivalents in the absence of information about the relative importance of the two objectives, as in [62], [63]. This method seeks solutions that exhibit a high performance in all dimensions. This set of solutions is known as Pareto-optimal front, and, in our case, represents the best known trade-off between network cost and coverage level. This strategy brings the benefit of quantifying the non-linear relationship between cost and coverage, allowing defining the network cost as a function of the coverage level, being thus extremely useful to plan an efficient and progressive deployment of a DVB-H network. In the last years, genetic algorithms have been successfully applied to solve multi-objective optimization problems due to their intrinsic advantages compared to other techniques [64]. Genetic algorithms are based on the selection mechanisms of the Nature by which the most fit individuals of a population survive. Multi-objective genetic algorithms try to find, ideally, a set of solutions uniformly distributed along the Pareto-optimal front. This is the approach adopted in this dissertation.

Regarding planning hybrid cellular and DVB-H networks, a first approach to automatic planning of hybrid DVB-H and 3G networks is presented in [43], where DVB-H cells are placed over an existing 3G network by using the realistic network traffic information [65]. Furthermore, the necessary transmit power is estimated. The gain of the hybrid network is measured in terms of the overall data rate required. In [66], the same approach is employed to reduce the electromagnetic exposure.

\section{RRM in Hybrid Cellular and Broadcasting Systems}

Radio resource management (RRM) plays a major role in quality of service (QoS) provisioning for mobile wireless communication systems. 
RRM techniques control the amount of resources (frequency and/or time channels, codes, transmitted power, etc.) assigned to each user subject to some QoS constraints (bit rates, error probabilities, delays, etc.) for a given objective. RRM objectives can be the maximization of network throughput or number of satisfied users, among many others.

The issue of radio resource management in a hybrid cellular and DVB-H system from a network access selection point of view has been recently treated in the literature. Results show significant gain in spectrum efficiency compared to traditional systems. The basic idea is to aggregate the requests for a given content arriving over a certain period of time, and then serve them with a multicast/broadcast transmission. In [67] a load balancing algorithm for access management in a hybrid GPRS and DVB-H network is introduced and evaluated. In [68] a queuing model is derived, and it is used to analytically study the performance of the system in terms of stability and service response time. In [69] it is proposed a framework for access selection in a system where several multicast/broadcast networks exist. The goal is to maximize the system profit while satisfying users' QoS requirements. Finally, in [70] an analytical model is derived to analyze the optimum time period to group user requests, taking into account the trade-off between user blocking probability and user reneging probability. The goal is to maximize the percentage of served users.

The main contributions of the above mentioned papers are the proposals of RRM frameworks based on dynamic network access selection from a queuing theory point of view. However, radio channel characteristics and user mobility are not taken into account, and they assume perfect coverage. Hence, they do not consider the possibility of jointly using the cellular and the DVB-H networks to transmit the same service.

An interesting approach is the one proposed in [71] and [72], where a cost-based framework for the resource management in hybrid cellular-broadcasting systems with adaptive data rate capabilities is introduced. The service considered is delivery of multimedia items (files). The RRM goal is the minimization of the service delivery cost, which is proportional to the time of bearer usage. For that purpose the measures of cost per time unit when using the broadcasting and cellular bearers are introduced. The proposed solution is to search for the optimum set of users that should be allocated to the broadcasting radio access (and the respective data rate for serving them), in order to minimize the overall transmission cost, assuming that the remaining users are served through the cellular network. Results show that it is possible to achieve significant cost reductions compared to the cases when only one network is employed in some cases. This depends on the number of users and the ratio between cost per time unit of using the broadcasting and the cellular radio access [71]. Moreover, it is shown that the cost savings are not significantly affected by the accuracy of the estimated data rates when the number of users is large, which may allow using statistical information to estimate the spatial distribution of the users and their average reception conditions [72]. Again, an error free transmission of the files is assumed, without taking into account that mobile users experiment temporary errors due to shadowing and fast fading. In this dissertation we will use this framework but taking into account the particularities of DVB-H, MBMS and HSDPA (e.g., DVB-H does not provide adaptive modulation). 
Regarding radio resource management issues in $3 \mathrm{G}+$ cellular networks, a lot of work has been done on HSDPA, see e.g., [3] and references therein, but there is not that much information on MBMS. Especially there is no public literature on the gain that can be achieved using HSDPA repairing errors of the MBMS transmissions. A good overview about the delivery of broadcast services in $3 \mathrm{G}+$ networks can be found in [11], where the capabilities of both HSDPA and MBMS to provide mobile TV services are discussed. It is stated that only two users per cell in average are needed to achieve a more efficient utilization in terms of power usage with MBMS than with HSDPA in case signals from different cells can be combined; and between five and ten users otherwise. In [73] and [74] it is shown that only TV channels with high viewing probability should be broadcasted with MBMS, while the rest should be offered on-demand using HSDPA. An analytical framework to derive the optimal number of MBMS broadcast bearers in a hybrid MBMSHSDPA mobile TV cellular system can be found in [75].

The most relevant literature regarding RRM in MBMS are [77] and [76], where it is investigated the trade-off between application layer FEC and physical layer FEC for streaming and file download services. For download services it is shown that it is more efficient to employ low transmission power and moderate coding at the physical layer, which results in relatively large radio packet loss rates that are compensated by using a substantial amount of application layer FEC. Similar findings apply for streaming delivery. However, in this case the gain is smaller to support real-time delivery with small zapping times.

Another interesting reference is [78]. It discusses the trade-off between application layer FEC protection and successive file repair for download delivery services. The benefits of using Raptor coding in the file repair phase are outlined when the repair user group is significantly large, as it also enables p-t-m repair transmissions. It is shown that a $100 \%$ error free initial transmission of the files is generally not efficient, and it proposes a repair scheme combining both $\mathrm{p}-\mathrm{t}-\mathrm{p}$ and $\mathrm{p}-\mathrm{t}-\mathrm{m}$ transmissions to minimize the overall transmission time and the resource usage. However, the transmission errors experienced by the users are modeled with a rather basic model based on an exponential distribution.

\subsection{Dissertation Outline and Contributions}

The dissertation is structured into six main chapters and two appendices as follows:

Chapter 2 describes the different link layer FEC (LL-FEC) and application layer FEC (AL-FEC) mechanisms possible in DVB-H in detail, and reviews fundamentals for forward error correction at the upper layers. Chapter 3 presents illustrative performance evaluation results with field measurements of intra-burst MPE-FEC and multi-burst FEC for different reception conditions and transmission configurations. It also presents simulation results quantifying the reduction in the fading margin required to cope with fast fading and shadowing that can be achieved with multi-burst FEC compared to MPE-FEC. Chapter 4 is devoted to DVB-H network planning and deployment issues. It describes a methodology to estimate the coverage for multi-burst FEC DVB-H services in realistic scenarios based on dynamic system-level simulations. In addition, it presents studies on the feasibility 
and scalability of deploying DVB-H on the existing wireless infrastructure, it describes a network planning algorithm based on a multi-objective genetic algorithm to find cost minimized and coverage maximized network configurations, and it discusses the implications of using gap-fillers instead of synchronized transmitters. Chapter 5 deals with radio resource management issues for filecasting services in hybrid DVB-H/3G+ systems. It analyzes the cases when both networks are used separately, and the case when they are used jointly, using the cellular network to repair errors of the DVB-H broadcast transmissions. In Chapter 6 conclusions to the entire dissertation are drawn, and the possibilities of performing a progressive deployment of the DVB-H infrastructure are discussed. Suggestions on future research topics are also provided.

Appendix A provides a description of the DVB-H performance evaluation methodology using field measurements and dynamic simulations. Measurements were performed for vehicular and pedestrian reception conditions in the DVB-H pilot networks of the universities of Turku (Finland) and Braunschweig (Germany). The measurement set-up and the measurements performed are described in this appendix. The appendix also describes the dynamic DVB-H system-level simulator developed during this dissertation, which have been used to obtain all DVB-H simulation results, and the system models employed in the cellular simulations. Finally, Appendix B contains a theoretical and simulation analysis on the gain that can be achieved with multi-slot FEC in a generic time division multiplexing broadcast system.

The key contributions of the dissertation in each of the chapters and appendixes are summarized next, including references to the most relevant dissertation publications of the author. The complete list of publications can be found in the next section.

\section{Chapter 2 - Upper Layer FEC in DVB-H}

The main contribution of this chapter is the proposal of using AL-FEC with Raptor coding to perform a multi-burst protection of the transmission for DVB-H streaming services [79]. The idea was first introduced and evaluated in [80], where it was proposed to transmit additional bursts with repair data after the original data bursts in order to increase the chances of receiving the information correctly ${ }^{22}$. However, when comparing this approach with file download services in the same scenario in [44], it was found that the FEC efficiency can be considerably improved by delivering the streaming content as a succession of larger data blocks, exploiting the time diversity of the mobile channel. In this way, the transmission robustness can be improved delivering the streaming content as a succession of larger source blocks transmitted over more bursts keeping the proportion of data employed for error correction. The introduction of this technique would require minor changes in the content delivery protocols of the current IPDC specifications. The framework would be aligned with the framework defined by the IETF and already adopted in 3G MBMS [56]. Furthermore, it could be introduced as a software update in existing networks and terminals.

\footnotetext{
${ }^{22}$ It should be noted that the simple retransmission of a burst (with or without MPE-FEC) is not an efficient solution, since it implies that users may receive duplicate packets which are useless.
} 
In parallel to this dissertation work, a multi-burst FEC framework for streaming services at the link layer was defined in the new DVB-SH standard known as MPE-iFEC (MPE inter-burst FEC) [81]. This framework is also defined backwards-compatible with DVB-H, and it allows employing Raptor coding in a similar way than the proposed by the author in [79], but at the link layer. It should be pointed out that the performance in both cases (link and application layers) is practically the same, and only some implementation and signaling specific aspects differ. The work performed by the author of this dissertation on multi-burst FEC in DVB-H lead to the invitation to participate in the DVB standardization forum in the completion on the DVB bluebook ${ }^{23}$ on upper layer FEC in DVB systems as an invited expert [46]. Chapter 2 contains most of the contributions of the author to the bluebook.

Finally, in Chapter 2 we describe the benefits of using AL-FEC in a hybrid cellular and DVB-H system. The use of AL-FEC is key to enable an easy and efficient interworking between the DVB-H and the cellular network. This cannot be achieved if multi-burst FEC is implemented in lower layers. Within this context, a framework for affordable IP datacast over hybrid cellular and DVB-H systems using AL-FEC based on a progressive and cost-efficient deployment of the DVB-H infrastructure is proposed in [38], which can be considered the overall framework of this dissertation.

\section{Chapter 3 - Multi-Burst FEC in DVB-H}

Chapter 3 contains almost exclusively novel material on the performance evaluation of multi-burst FEC in DVB-H with background information kept to a minimum. At the day of writing this dissertation there are no other published results on the topic using field measurements in real networks or focusing on streaming services. Publications on the performance of multi-burst FEC for streaming delivery are thus especially novel, see e.g., [79] and [82].

In this chapter we also show that multi-burst FEC yields a minimum fading margin gain due to fast fading, which can be increased due to shadowing depending on the shadowing correlation between reception conditions of the bursts jointly encoded [83]. Previous literature on the topic, including the DVB implementation guidelines for file delivery with AL-FEC [57], and complementary studies [59], state that the gain stems only due to shadowing.

This chapter can be considered as a set of performance evaluation guidelines for multiburst FEC DVB-H services as a function of the different transmission parameters and reception conditions.

\footnotetext{
${ }^{23}$ DVB bluebooks and sent to ETSI (European Telecommunications Standards Institute) for publication as formal standards or implementation guides.
} 


\section{Chapter 4 - DVB-H Infrastructure Deployment}

The main contribution of this chapter is a methodology to estimate the coverage of multiburst FEC services in realistic scenarios based on dynamic system-level simulations [84], [85]. This problem has not been previously addressed in the literature. As the performance of multi-burst FEC depends on the mobility and trajectory of the users, accurate received signal strength predictions and realistic mobility patterns are required, together with an accurate and computationally efficient DVB-H performance model such as the one described in Appendix A.

Other contributions of this dissertation on DVB-H network planning are the feasibility [36], scalability [37], and minimal cost [40] studies on the deployment of DVB-H on existing wireless infrastructure for digital broadcasting and cellular networks. Nowadays results presented in these publications are acknowledged as intrinsic issues of DVB-H, but they were the first public literature pointing out that reuse of the existing wireless infrastructure is a must to reduce the DVB-H network deployment cost. These publications have been referenced by several authors, e.g., [86] and [87]. Moreover, they helped to lay the foundations of this dissertation.

\section{Chapter 5 - RRM in Hybrid Cellular and DVB-H Systems}

This dissertation addresses novel regarding radio resource management issues for file delivery in hybrid DVB-H/3G+ systems and in 3G+ networks where users that do not receive the file after the initial multicast/broadcast transmission are served with unicast HSDPA connections. This fact has not been previously considered in the literature, where it is more often considered that users are served using exclusively a single radio access. Results have been obtained with system-level dynamic simulations in realistic scenarios.

The main contributions of this chapter are the evaluation of the gain (in terms of increased cell capacity) that can be achieved using HSDPA for error repair of the MBMS file download transmissions [88]; and the evaluation of the cost delivery savings that can be achieved using HSDPA and MBMS for error repair of the DVB-H file download transmissions. These results have not been published yet. The chapter also presents novel contributions for radio resource management for file delivery services in DVB-H networks, [44], [89].

In addition to this work, the author also participated in the development of RRM algorithms based on Hopfield neural networks (HNN) for 3G networks [90], [91], [92], and for heterogeneous $3 \mathrm{G}$ and WLAN networks [93]. These publications are not included in the dissertation. 


\section{Appendix A - DVB-H Performance Evaluation Methods}

The main contribution of this appendix is the proposed DVB-H system-level simulator [94]. These simulators are widely used in cellular networks to investigate QoS and RRM issues, but they have not been used up to date for DVB-H. With the system-level simulator it is possible to simulate a DVB-H network over time, being able to study the overall system performance in a very detailed manner. They can be used as a complement of traditional coverage planning tools for broadcasting networks to estimate the overall QoS perceived by the users dynamically over time, giving a better understanding of the network behavior.

In the appendix a general simulation structure is described along with the main models required. The key element is a DVB-H performance model. In this dissertation we consider a DVB-H physical layer performance model based on parameterized 4-state Markov processes originally proposed in [95]. More details on the model can be found in [96]. This dissertation work contributed to the validation of the model with laboratory and field measurements [97].

\section{Appendix B - Multi-Slot FEC in TDM Broadcast Systems}

This appendix quantifies the reduction in the fading margin required to cope with shadowing that can be achieved in a time division multiplexing system with multi-slot FEC as a function of the code rate and the number of jointly encoded slots [98]. For the case when there is no shadowing correlation between consecutive slots results have been derived analytically, whereas under correlation conditions results have been obtained by simulations. Moreover, results have been validated with DVB-H results obtained in Chapter 3. The motivation for this work was to develop a generic framework considering both streaming and file download services, and correlated and uncorrelated shadowing. Although specific use cases have been analyzed in the literature, e.g., [58], [59], a complete framework was missing. Finally, it should be pointed out that although the problem description has been tailored to broadcast systems such as DVB-H/SH, results also apply to any TDM wireless communication system (p-t-p or p-t-m). 


\subsection{List of Publications}

\section{Standardization Reports}

1. DVB bluebook, "Upper Layer Forward Error Correction in DVB," work in progress.

\section{International Journal Articles}

1. D. Gómez-Barquero, A. Bria, J. Zander, and N. Cardona, "Affordable Mobile TV Services in Hybrid Cellular and DVB-H Systems," IEEE Network - special issue on Convergence of Internet and Broadcasting Systems, vol. 21, no. 2, pp. 34-40, March-April 2007.

2. D. Gómez-Barquero and A. Bria, "Error Repair for Broadcast Transmissions in DVB-H Systems," Wiley Wireless Communications and Mobile Computing, vol. 9, no. 6, pp. 733-744, June 2009.

3. D. Gómez-Barquero, D. Gozálvez, and N. Cardona, "Application Layer FEC for Mobile TV Delivery in IP Datacast over DVB-H Systems," IEEE Transactions on Broadcasting - special issue on IPTV Multimedia Broadcasting, vol. 55, no. 2, pp. 396-406, June 2009.

4. D. Gómez-Barquero, A. Fernández-Aguilella, and N. Cardona, "Multicast Delivery of File Download Services in Evolved 3G Mobile Networks with HSDPA and MBMS," to appear in IEEE Transactions on Broadcasting.

5. D. Gómez-Barquero and J. Poikonen, "Development and Applications of a DVB-H SystemLevel Simulator," submitted to IEEE Transactions on Broadcasting.

6. D. Gómez-Barquero, D. Gozálvez, P. Olivas and N. Cardona, "Multi Burst Forward Error Correction for Mobile TV Services in DVB-H," submitted to IEEE Communications Magazine.

7. D. Gómez-Barquero and P. Unger, "Coverage Estimation for Multi-Burst Forward Error Correction Services in DVB-H Networks," submitted to IEEE Transactions on Broadcasting.

8. D. Gómez-Barquero and N. Cardona, "Fading Margin Gain due to Multi-Slot FEC in TDM Broadcast Systems," submitted to IEEE Transactions on Broadcasting.

9. D. Gozálvez, D. Gómez-Barquero, and T. Stockhammer, "AL-FEC for Improved Mobile Reception of MPEG-2 DVB-T Transport Streams," submitted to International Journal of Digital Multimedia Broadcasting - special issue on Convergence of Digital TV Systems and Services.

10. D. Calabuig, J. F. Monserrat, D. Gómez-Barquero, and O. Lázaro, "User Bandwidth Usage - Driven HNN Neuron Excitation Method for Maximum Resource Utilization within Packet - Switched Communication Networks," IEEE Communications Letters, vol. 10, no. 11, pp. 766-768, Nov. 2006.

11. D. Calabuig, J. F. Monserrat, D. Gómez-Barquero, and O. Lázaro, "An Efficient Dynamic Resource Allocation Algorithm for Packet-Switched Communication Networks Based on Hopfield Neural Excitation Method," Elsevier Neurocomputing, vol. 71, no. 16, pp. 3439-3446, Oct. 2008.

12. D. Calabuig, J. F. Monserrat, D. Gómez-Barquero, and N. Cardona, "A Delay-Centric Dynamic Resource Allocation Algorithm for Wireless Communication Systems based on HNN," IEEE Transactions on Vehicular Technology, vol. 57, no. 6, pp. 3653-3665, Nov. 2008. 


\section{International Conference Papers}

1. D. Gómez-Barquero and A. Bria, "Feasibility of DVB-H Deployment on Existing Wireless Infrastructure," Proc. International Workshop on Convergent Technologies (IWCT), Oulu, Finland, 2005.

2. A. Bria and D. Gómez-Barquero, "Scalability of DVB-H Deployment on Existing Wireless Infrastructure," Proc. IEEE Personal, Indoor and Mobile Radio Communications (PIMRC), Berlin, Germany, 2005.

3. D. Calabuig, D. Gómez-Barquero, J. F. Monserrat, and N. García, "A Dynamic Resource Allocation Algorithm for WCDMA Systems with Delay Constraints based on Hopfield Neural Networks," Proc. Trends in Radio Resource Management, Barcelona, Spain, 2005.

4. J. F. Monserrat, N. Cardona, D. Gómez-Barquero, and L. Rubio, "Radio Performance Evaluation of an Enhanced UTRAN Architecture," Proc. Wireless Personal Multimedia Communication (WPMC), Aalborg, Denmark, 2005.

5. D. Gómez-Barquero, A. Bria, J. F. Monserrat, and N. Cardona, "Minimal Cost Planning of DVB-H Networks on Existing Wireless Infrastructure," Proc. IEEE Personal, Indoor and Mobile Radio Communications (PIMRC), Helsinki, Finland, 2006.

6. D. Gómez-Barquero and A. Bria, "Application Layer FEC for Improved Mobile Reception of DVB-H Streaming Services," Proc. IEEE Vehicular Technology Conference (VTC) Fall, Montreal, Canada, 2006.

7. D. Gómez-Barquero and A. Bria, "Repair Mechanisms for Broadcast Transmissions in Hybrid Cellular and DVB-H Systems," Proc. IEEE International Symposium on Wireless Communications Systems (ISWCS), Valencia, Spain, 2006.

8. D. Calabuig, J. F. Monserrat, D. Gómez-Barquero, and N. Cardona, "Hopfield Neural Network Algorithm for Dynamic Resource Allocation in WCDMA Systems," Proc. IEEE International Symposium on Wireless Communications Systems (ISWCS), Valencia, Spain, 2006.

9. J. F. Monserrat, D. Gómez-Barquero, D. Calabuig, L. Rubio, and N. Cardona, "Evaluation of Soft Handover Micro Diversity Gain on the UMTS System Capacity and QoS," Proc. IEEE Personal, Indoor and Mobile Radio Communication (PIMRC), Helsinki, Finland, 2006.

10. D. Gómez-Barquero, D. Calabuig, J. F. Monserrat, N. Cardona, N. García, and J. PérezRomero, "Hopfield Neural Network Algorithm for Joint Dynamic Resource Allocation in Heterogeneous Wireless Networks," Proc. NEWCOM and ACORN Joint Workshop, Vienna, Austria, 2006.

11. D. Gómez-Barquero, D. Calabuig, J. F. Monserrat, N. García, and J. Pérez-Romero, "Hopfield Neural Network - based Approach for Joint Dynamic Resource Allocation in Heterogeneous Wireless Networks," Proc. IEEE Vehicular Technology Conference (VTC) Fall, Montreal, Canada, 2006.

12. D. Gómez-Barquero and A. Bria, "Forward Error Correction for File Delivery in DVB-H," Proc. IEEE Vehicular Technology Conference (VTC) Spring, Dublin, Ireland, 2007.

13. J. Poikonen and D. Gómez-Barquero, "Validation of a DVB-H Dynamic System Simulator using Field Measurements," Proc. IEEE Broadband Multimedia Systems and Broadcasting, Las Vegas, USA, 2008.

14. D. Gómez-Barquero and J. Poikonen, "Filecasting for Streaming Content in IP Datacast over DVB-H Systems," Proc. IEEE Broadband Multimedia Systems and Broadcasting, Las Vegas, USA, 2008. 
15. D. Gómez-Barquero, A. Fernández-Aguilella, and N. Cardona, "Multicast Delivery of File Download Services in 3G Mobile Networks with MBMS," Proc. IEEE Broadband Multimedia Systems and Broadcasting, Las Vegas, USA, 2008.

16. D. Gozálvez, D. Gómez-Barquero, and N. Cardona, "Performance Evaluation of the MPE-iFEC Sliding RS Encoding for DVB-H Streaming Services," Proc. IEEE Personal, Indoor and Mobile Radio Communications (PIMRC), Cannes, France, 2008.

17. P. Unger and D. Gómez-Barquero, "Coverage Gain Estimation for Multi-Burst Forward Error Correction in DVB-H Networks," Proc. IEEE Broadband Multimedia Systems and Broadcasting, Bilbao, Spain, 2009.

18. D. Gozálvez, D. Gómez-Barquero, and T. Stockhammer, "Mobile Reception of DVB-T Services by means of Application Layer FEC Protection," Proc. IEEE Broadband Multimedia Systems and Broadcasting, Bilbao, Spain, 2009.

\section{COST2100 Technical Documents}

1. D. Gómez-Barquero, A. Bria, F. Álvarez, and N. Cardona, "File Delivery over IP Datacast over DVB-H Systems - an Overview," Proc. COST2100, TD 07-033, Lisbon, Portugal, 2007.

2. D. Gómez-Barquero, A. Fernández-Aguilella, and N. Cardona, "Multicast Delivery of File Download Services in E3G Mobile Networks - an Overview," Proc. COST2100, TD 07-372, Duisburg, Germany, 2007.

3. J. Poikonen and D. Gómez-Barquero, "Parameterized Markov Processes for Modelling the Performance of the DVB-H Physical Layer for DVB-H System Level Simulations," Proc. COST2100, TD 07-374, Duisburg, Germany, 2007.

4. J. Poikonen and D. Gómez-Barquero, "On Dynamic DVB-H System-Level Simulations," Proc. COST2100, TD 08-447, Wroclaw, Poland, 2008.

5. D. Gómez-Barquero and J. Poikonen, "On the File Delivery for Streaming Content in IP Datacast over DVB-H Systems," Proc. COST2100, TD 08-454, Wroclaw, Poland, 2008.

6. D. Gozálvez, D. Gómez-Barquero, and N. Cardona, "MPE-iFEC Sliding RS Encoding for DVB-H Streaming Services," Proc. COST2100, TD 08-538, Trondheim, Norway, 2008.

7. D. Gómez-Barquero and P. Unger, "On the Fading Margin Gain due to Multi-Slot FEC in TDMA Broadcast Systems," Proc. COST2100, TD 08-641, Lille, France, 2008.

8. P. Unger and D. Gómez-Barquero, "Coverage Estimation for Multi-Burst Forward Error Correction in DVB-H Networks," Proc. COST2100, TD 08-650, Lille, France, 2008.

9. D. Gozálvez, D. Gómez-Barquero, and T. Stockhammer, "Mobile Reception of DVB-T Services by means of Application Layer FEC Protection," Proc. COST2100, TD 09-718, Braunschweig, Germany, 2009. 


\section{National Journal Articles}

1. J. F. Monserrat, D. Calabuig, D. Gómez-Barquero, and N. Cardona, "Cronoejecución y Gestión Adaptativa de Paquetes en el Sistema de Comunicaciones Móviles UMTS," Revista Gerencia Tecnológica Informática, vol. 5, no. 11, pp. 11-20, April 2006.

2. D. Gómez-Barquero, A. Salieto, A. P. García, J. F. Monserrat, and N. Cardona, "Planificación de una Red DVB-H en Entorno Urbano," Revista Sistemas y Telemática, no. 9, pp. 13-34, April 2007.

3. A. Fernández-Aguilella, D. Gómez-Barquero, and N. Cardona, "Transmisión Híbrida UnicastMulticast de Servicios de Descarga de Ficheros en Redes Móviles 3G con MBMS," Revista Gerencia Tecnológica Informática, vol. 7, no. 17, pp. 13-22, April 2008.

4. J. Martín, D. Gómez-Barquero, A. Salieto, and N. Cardona, "Planificación de Redes de TDT Móvil en Entornos Urbanos mediante Simulated Annealing," Revista Gerencia Tecnológica Informática, vol. 7, no. 17, pp. 73-83, April 2008.

\section{National Conference Papers}

1. D. Gómez-Barquero, J. F. Monserrat, and N. Cardona, "Planificación y Despliegue de Red Eficiente de DVB-H," Jornadas Telecom I+D, Madrid, Spain, 2005.

2. D. Gómez-Barquero and N. Cardona, "Transmisión de Servicios Podcast en DVB-H,” Jornadas Telecom $I+D$, Madrid, Spain, 2006.

3. D. Gómez-Barquero, A. Salieto, A. P. García, J. F. Monserrat, and N. Cardona, "Planificación de una Red DVB-H en la Ciudad de Valencia," Jornadas Telecom I+D, Madrid, Spain, 2006.

4. D. Gómez-Barquero, A. Salieto, J. Martín, and N. Cardona, "Planificación y Despliegue de Redes de TDT Móvil DVB-H utilizando Gap-fillers," Jornadas Telecom I+D, Valencia, Spain, 2007.

5. D. Gómez-Barquero, A. Fernández-Aguilella, and N. Cardona, "Transmisión Multicast de Servicios de Descarga de Archivos en Redes Móviles 3G," Jornadas Telecom I+D, Valencia, Spain, 2007.

6. D. Gozálvez, D. Gómez-Barquero, and N. Cardona, "Transmisión de Servicios de Televisión Digital Móvil en Redes DVB-SH,” Jornadas Telecom I+D, Valencia, Spain, 2007.

7. D. Gómez-Barquero, D. Gozálvez, P. F. Gómez, and N. Cardona, "Desarrollo y Aplicaciones de un Simulador Dinámico de TDT Móvil DVB-H," Jornadas Telecom I+D, Bilbao, Spain, 2008 .

8. D. Gozálvez, D. Gómez-Barquero, F. Camaro, and N. Cardona, "MPE-iFEC para la Transmisión de Servicios de Streaming en Redes DVB-H," Jornadas Telecom I+D, Bilbao, Spain, 2008.

9. J. Martín, A. Salieto, D. Gómez-Barquero, G. Roig, A. P. García, and N. Cardona, "Herramienta de Planificación de Redes de TV Móvil DVB-H/SH," Jornadas Telecom I+D, Bilbao, Spain, 2008. 



\section{Chapter 2}

\section{Upper Layer Forward Error Correction in DVB-H}

As DVB-H only provides a unidirectional downlink channel, error repair can only be achieved transmitting additional repair information (parity data), such that the receivers can detect and possibly correct errors occurred during the transmission. DVB-H inherits the physical layer FEC of the DVB-T standard, which corrects errors within MPEG-2 TS packets [99]. Unfortunately, DVB-T was primarily designed for fixed rooftop reception with a very short time interleaving (couple of milliseconds), being very vulnerable to fast fading in mobile channels. To increase the robustness of the DVB-H transmission while maintaining compatibility with DVB-T, additional FEC schemes can be employed in the link and application layers to generate repair data which is transmitted along with the original source IP information [46]. This way the original multimedia service is not modified and terminals without upper layer FEC decoding capabilities can still receive the service without the additional FEC protection. In short, upper layer FEC (UL-FEC) schemes in DVB-H aim to enlarge the time interleaving duration of the information to take advantage of the time diversity of the mobile channel.

The drawback of sending additional repair data is not only a reduction in the system capacity, but also an increase of the system latency. The presence of latency in a unidirectional broadcast system affects the user experience by delaying the initial reproduction of the services, increasing the end-to-end delay, but also the channel switching time (zapping time in case of mobile TV). In IP datacast systems multimedia content can be delivered as a streaming service or as a file download service to the end user [54]. These two service categories are of very different nature and have different quality of service (QoS) requirements in terms of residual error rate and latency constraints. In this chapter we first highlight the differences between streaming and file download services in DVB-H in Section 2.1. We review upper layer FEC fundamentals in Section 2.2, and describe in detail the different link layer FEC (LL-FEC) and application layer FEC (AL-FEC) mechanisms available in DVB-H in Section 2.3. The benefits of using AL-FEC in a hybrid cellular and DVB-H system are explained in Section 2.4. Section 2.5 concludes the chapter. 


\subsection{Mobile Multimedia Broadcasting Services and QoS in DVB-H}

\section{Streaming vs. File Download}

Generally speaking, mobile multimedia broadcasting services can be delivered either as a streaming service or as a file download service, or a combination of both. The main difference is that streaming delivery must be accomplished in such a way that the multimedia application at the receiver can make use of earlier received information while the reception is still in progress. The most representative service is mobile TV. In contrast, download applications start processing the information after the entire file has been received without early play out features. File download services (also called filecasting) can be used for most content types, including multimedia clips, high quality music files, digital newspapers, software download, etc.

Another important difference between streaming and file download services is that streaming services may tolerate occasional data errors, whereas file download services typically require an error-free reception of the files, as even a single bit error can corrupt the entire file and make it useless to the receiver. Nevertheless, TV services generally have very high demands in terms of tolerable packet error rates, as consumers are used to and expect very high video quality. Although video and audio decoders may sustain packet losses to some extent before they break down entirely, unpredictable decoder behavior and residual visible errors remain, degrading the quality of the service severely.

\section{Streaming Services in DVB-H}

For streaming services generally a continuous data flow of audio, video and subtitling is transmitted to the terminals and is directly consumed by the users. In IPDC the streaming delivery protocol is RTP (Real-time Transport Protocol) [54]. RTP is designed to carry real-time content, providing time stamps, identification of the type of payload, sequence numbering, and control mechanisms to synchronize different streams while respecting their relative timing properties [100]. The sequence numbers included in RTP allow the receiver to reconstruct the original packet sequence, but RTP does not guarantee delivery or prevent out-of-order delivery.

Streaming services are real-time services with hard latency constraints. According to the nature of the transmitted content, it can be distinguished between live transmissions, where it is desirable to minimize the end-to-end delay in the system, and services where a delay between data delivery in the terminal and visualization of the content is acceptable (e.g., video clips, soap opera episodes). In any case for mobile TV it is crucial to provide a short zapping time, as it has a significant impact on the QoS perceived by the users. The zapping time is defined as the time that a user has to wait to start watching the chosen TV channel since the instant of switching channels. Subjective studies have shown that zapping times of less than 500 milliseconds are perceived as instantaneous, whereas more than two seconds are felt as annoying [101]. In DVB-H there is some inevitable delay due to its discontinuous transmission pattern, as users need to wait for the first burst of the new channel, but it also depends on the UL-FEC mechanism employed. We discuss zapping 
time issues in DVB-H and solutions to reduce and hide it from the perception of the users in Section 2.3.4.

In DVB-H bursts are transmitted with time constraints and terminals play the information received in the last burst until the next one is received, in such a way that users do not notice the discontinuous transmission. If one burst is lost, the multimedia stream is interrupted until a burst is again received. An unambiguous measure of the quality of an audiovisual stream transmitted over a wireless network is very difficult to achieve, as it embeds subjective aspects such as quality expectation or relative importance of the audio and the video which are content-dependent. Hence, large-scale subjective testing is ultimately the only accurate mean for audiovisual quality measurement. Due to these reasons, different objective failure points or quality of restitution (QoR) criteria are defined in DVB-H in order to characterize the operative limit of a streaming service under mobility conditions [102].

The DVB-T standard specifies the quasi-error-free (QEF) reception criterion, which means one uncorrected error event per hour, corresponding to a bit error rate (BER) of $10^{-11}$ at the input of the MPEG-2 demultiplexer. Due to high variations occurring in a mobile channel, the QEF criterion is not suitable for mobile multimedia broadcasting. Therefore, less restrictive error criteria have been accepted than for fixed reception. Typically, a 5\% burst error rate is considered as the degradation point for streaming services in DVB-H [14]. However this metric does not take into account that it is possible to receive bursts partially (i.e., some IP packets may be correctly received). The metric that reflects most accurately the amount of correctly received information is the IP packet error ratio (IP PER), which represents the percentage of erroneously received IP packets. Another metric is the erroneous second ratio (ESR), which represents the percentage of seconds that contain errors. Burst error rate, IP PER and ESR only account for the overall transmission errors experienced by the users. However the time distribution of the errors also affects the QoS of a streaming service perceived by the users. This can be taken into account with the ESR5(20) ratio, which represents the percentage of time intervals of twenty seconds with at most one second with errors (i.e., $5 \%$ errors).

\section{File Download Services in DVB-H}

For file download services a finite amount of data is delivered and stored into the terminals as a file. IPDC uses FLUTE (File Delivery over Unidirectional Transport Protocol) as the file delivery protocol [54]. FLUTE is designed to deliver files in IP-based unidirectional environments, being particularly suited to multicast/broadcast networks [103]. Both single file transfers and data carousel sessions where the file is repeatedly transmitted are supported. A brief overview of FLUTE can be found in Section 2.2.3.

In DVB-H terminals first store the correctly received information in each burst associated to the file until the complete file is available at the receiver before being accessed by applications. The main indicator in terms of QoS for these services is whether the user receives the file correctly or not. Additionally, the time required to receive the file is also of interest. 
For file download services delays are not as critical as for streaming services. It can be distinguished between files that need to be delivered on demand within a certain amount of time, and files that support background transfer in which users are not aware of the transmission. The latter enables "push and store" services, where the content can be delivered to the terminals beforehand and then played by the user providing the illusion of instant transmission. Filecasting applications itself may be set-up in different ways. In a scheduled distribution the start of the delivery is announced to the receiver population beforehand such that all interested receivers can join the download session at the appointed time. The session is generally terminated when the transmitter anticipates that a sufficient amount of receivers have successfully received the file. In contrast, in a carousel distribution receivers can join the download session at any point of time independently of other receivers in an asynchronous fashion. The transmission of the files is virtually unbounded and the receivers only leave the carousel when they have received the file. Two types of file carousel services can be distinguished, static and dynamic. Whereas the former delivers a file with the same content, in dynamic carousels individual files may change over time.

\subsection{Upper Layer Forward Error Correction}

\subsubsection{Overview}

Virtually any communication system employs some kind of FEC coding. The error correction capability of an FEC code depends on the distribution of the errors over time. Ideally, if the channel is memoryless and the errors are uniformly distributed it is possible to cope with error rates equal to the rate of parity data transmitted. However in practice long error bursts are common. This is particularly evident for wireless communication systems, where the transmission errors due to the impairments of the radio channel are heavily correlated due to the signal fading effects that mobile users experience in the field. But also the channel noise and impulse interference may also produce bursty errors.

As a consequence, if the data is sequentially transmitted, the FEC coding efficiency is reduced despite of a large error correcting capability. This performance degradation can be potentially solved using an interleaver to provide time diversity such that the coded data is distributed over time, at the expense of increased latency and more memory requirements at the receivers. The effectiveness of an interleaver mainly depends on the statistical correlation between the reception conditions of the interleaved data. The larger the interleaving depth, the better the interleaver can be expected to work. Indeed if a sufficiently large time interleaving is employed it is possible to cope with as many errors as in the best case (i.e., for an infinite interleaving the performance would be the same as for a memoryless channel). Nevertheless, it should be pointed out that if the code rate is not robust enough to correct the total number of errors there is no gain by increasing the interleaving, and the performance may even degrade. 


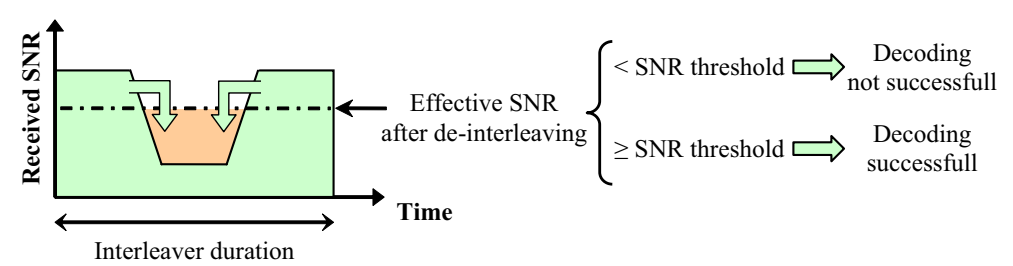

Figure 2.1: Time interleaving as time-averaging effect of the received SNR within the interleaving length.

Figure 2.1 shows a simplified illustration of the time-averaging effect of the received signal-to-noise ratio (SNR) as a result of time interleaving. Averaging out good and bad reception conditions it is possible to recover from reception instants with bad signal quality as soon as the mean SNR exceeds the required threshold. On the other hand, if the mean SNR is below the reception threshold, the reception can actually even worsen as errors are spread over a longer time span.

Generally speaking it can be distinguished between FEC mechanisms working at the physical layer and FEC mechanisms working at any upper layer above it, such as the link or application layer. Physical layer FEC codes work at the bit level and are traditionally implemented as part of the radio interface for wireless communication systems. They theoretically provide the most effective protection against channel noise, as they can exploit channel state information using soft decision decoding. In soft decision decoding each bit is assigned a confidence value ranging from a minimum confidence zero to a maximum confidence one, which can be used for more reliable probabilistic decoding. However, in practice the maximum time interleaving is rather small, in the order of few milliseconds or less, due to available on-chip memory and decoding complexity constraints (especially for handheld devices). The memory requirement for physical layer FEC decoding is directly proportional to the service data rate, the interleaving duration, and the fraction of parity data transmitted, which is usually large. As a consequence physical layer FEC is often combined with an upper layer FEC code, accepting a weaker performance in terms of level of protection, but achieving a better trade-off between overall system protection and system implementation. Also, in many applications there is a desire to extend a legacy bearer for other purposes, such as in the case with DVB-H and the DVB-T physical layer. In these cases, the extension of the physical layer FEC may not be possible, and additional FEC protection can be only provided in upper layers.

Upper layer FEC works in conjunction with physical layer FEC to produce a more efficient overall configuration. On the other hand, the optimization of the overall system FEC configuration becomes a cross-layer FEC configuration problem which is more difficult to solve. By operating above the physical layer it is possible to provide protection against longer losses with larger interleaving depths that physical layer cannot. The reason is that the high memory requirements allow support of host-based storage instead of memory-onchip. It should be also noted that physical layer FEC only overcomes the errors occurring in the radio interface, but it does not provide end-to-end reliability. 
If error protection is to be provided over several links at the same time, it is desirable to implement a FEC mechanism at the respective layer. This approach may also minimize the end-to-end delay. Additionally, it is also usually easier to implement service-specific solutions on higher layers.

Compared to physical layer FEC that corrects bit errors, upper layer FEC recovers from packet losses. Upper layer FEC codes are block codes that work with fixed-size symbols using erasure decoding. In erasure decoding each symbol is either considered correctly received or lost. Therefore, it is necessary to indicate whether each packet is correctly received or not (e.g., with checksums or cyclic redundancy check, CRC), such that the upper layer FEC decoder observes a virtual erasure channel. It should be noted that the mapping of packets to symbols and vice versa may be a one-to-one mapping, or it may be based on some specific mapping algorithms. If symbols and packets are not aligned, then packet losses at the physical layer typically result in higher symbol loss rates on the upper layers, as one single erroneous physical layer packet can cause the loss of several packets at the link and application layers. However, in practice as errors at the physical layer are usually correlated and several consecutive packets are lost [52], the symbol loss rate is only marginally higher than the packet loss rate.

Finally, it should be pointed out that FEC block codes can be also employed at the physical layer to correct physical layer packets. These codes are not considered as upper layer FEC, as they are an integral part of the physical layer.

\subsubsection{Definition of Upper Layer FEC}

An upper layer FEC code is any FEC code operating above the physical layer which performs erasure decoding. It can be distinguished between FEC codes operating in the application layer above the IP level, commonly known as application layer FEC (AL-FEC), and FEC codes operating within the link layer transport protocols below the IP level, commonly known as link layer FEC (LL-FEC).

An upper layer FEC encoder generates encoded symbols from a sequence of source packets that are combined in a source data block. Let $\mathrm{k}$ denote the number of source symbols with fixed size. The encoder generates $n \geq k$ encoding symbols of equal length that the source symbols, $k / n$ being the code rate. The rate of additional symbols compared to the original symbols, $(n-k) / k$, is also generally referred to as FEC overhead. If a so-called systematic code is applied, the first encoded symbols ${ }^{1}$ are simply the source symbols, and the rest consist of additional repair (parity) symbols . Obviously, if all source symbols are correctly received, no parity data is needed at all. Otherwise, with any subset of $r$ encoding symbols, such that $k \leq r \leq n$, the decoder can reconstruct the source data block. That is, it does not matter which symbols are received but that enough symbols are received correctly. The exact value of $r$ depends on the coding scheme used. It can be expressed as $r=(1+\varepsilon) \cdot k, \varepsilon$ being the reception overhead.

\footnotetext{
${ }^{1}$ In contrast, non-systematic codes produce a set of encoding symbols that do not contain a direct representation of the source symbols.
} 


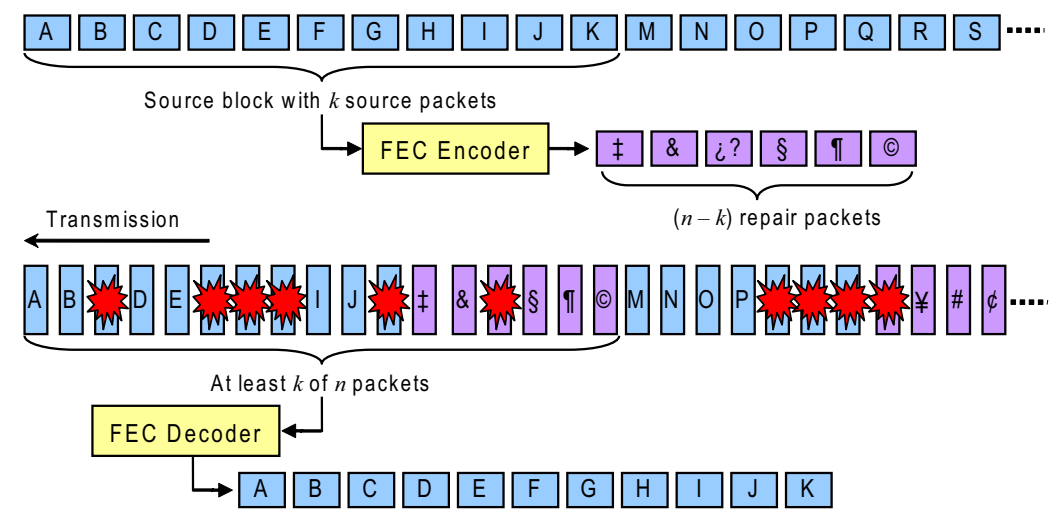

Figure 2.2: Upper layer FEC for streaming applications.

\subsubsection{Streaming and File Download Use Cases}

\section{Streaming Services}

Upper layer FEC can be used to provide streaming services over networks where packet data losses are common. Representative examples are high quality IPTV services over DSL networks [104], or in our case to improve the coverage for mobile TV DVB-H services.

With UL-FEC the source packets of the original multimedia stream are partitioned onthe-fly into consecutive source blocks of data which are treated independently. The time span over which the FEC is applied is generally referred to as protection period, and is typically in the range of several $10 \mathrm{~ms}$ to several seconds. From each of these blocks, a systematic FEC erasure encoder is utilized to produce repair packets to compensate for potential packet loss, which are sent along with the original source packets of the block as shown in Figure 2.2. To maintain the timing of the original multimedia stream, the source and repair packets associated with a source block must be transmitted over the same duration as the original data, requiring thus a faster data rate than the original stream in order to accommodate the repair packets. At the receiver, the received stream is also processed as blocks. The upper layer FEC decoder uses all the source and repair packets received from a particular source block to recover it. Ideally, the $k$ source packets of a source block can be reconstructed from any combination of $\mathrm{k}$ received source and repair packets. In this case the transmitted stream is resilient up to $n-k$ lost packets out of $n$ transmitted packets per source block. If the UL-FEC decoder fails, only correctly received source packets will be available for playback.

The major design parameters of the code are the FEC overhead and the protection period. They determine the trade-off between protection level against packet loss and the capacity reduction and the latency introduced. The system latency is increased due to the fact that the information is processed as blocks which are treated independently (at both the server and the terminals). Terminals must wait to receive all packets corresponding to the first source block to decode it. The latency corresponds to the amount of seconds of the original multimedia stream that are encoded jointly into a source block (protection period). 


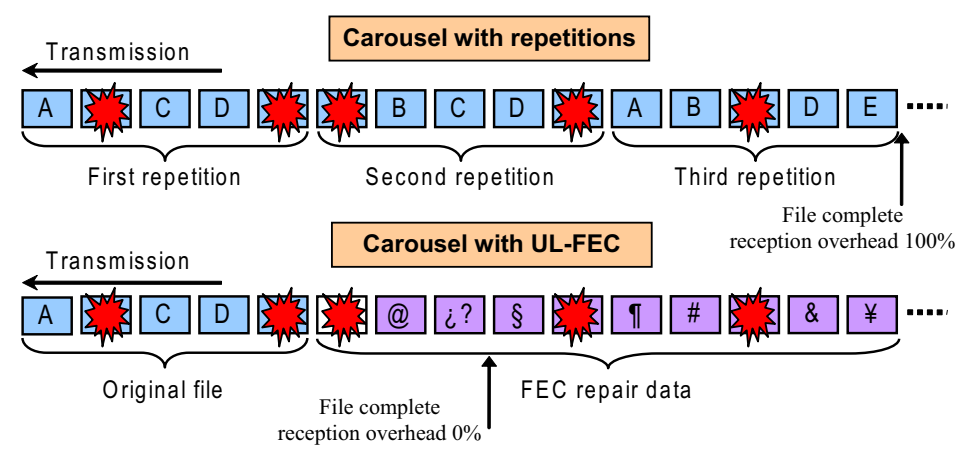

Figure 2.3: Carousel with repetitions without FEC vs. carousel with UL-FEC.

\section{Filecasting Services}

Upper layer FEC can be very beneficial for file download services in unidirectional multicast/broadcast environments where packet data losses are common. Its use can minimize the transmission duration and bandwidth while ensuring a reliably delivery.

In lossy environments the complete file may not be fully received by most receivers during the initial file transmission, since some packets may be lost. Without UL-FEC one common approach is to repeatedly transmit the file in a carousel until all receivers have completely received the file, see Figure 2.3. This is, however, not usually effective because each individual receiver must receive all packets of the file without error. If a receiver misses a single packet, it must wait until that specific packet is retransmitted and correctly received. The success of the file transmission for each receiver becomes probabilistic, resulting in the so-called "coupon collector problem" [105]. The key to this problem is to understand that it takes very little time to collect the majority of coupons, but it takes a long time to collect the last coupons. That is, receivers will likely receive more and more duplicate data from the carousel as they attempt to obtain their last fragment of the file. As a consequence, the total transmission time required so that all the receivers successfully obtain the file with a certain high probability increases drastically with the packet loss rate and the file size.

In contrast, if a single FEC code word is applied over the entire file with UL-FEC, then all source and repair packets are useful to the receivers as shown in the figure. It makes no difference which packets are received and thus receivers never receive duplicate data. In the "coupon collector problem" [105], it is irrelevant which specific coupons are received as long as a sufficient number of coupons is received. As a consequence, the time required to deliver files is reduced, and more content can be delivered. The file reception progresses at the arrival rate of data packets and no time is wasted waiting to receive specific packets.

The most prominent protocol to deliver files in unidirectional environments is the File Delivery over Unidirectional Transport protocol, commonly known as FLUTE [100]. It is carried over UDP/IP, and is independent of the IP version and the underlying link layers used. 


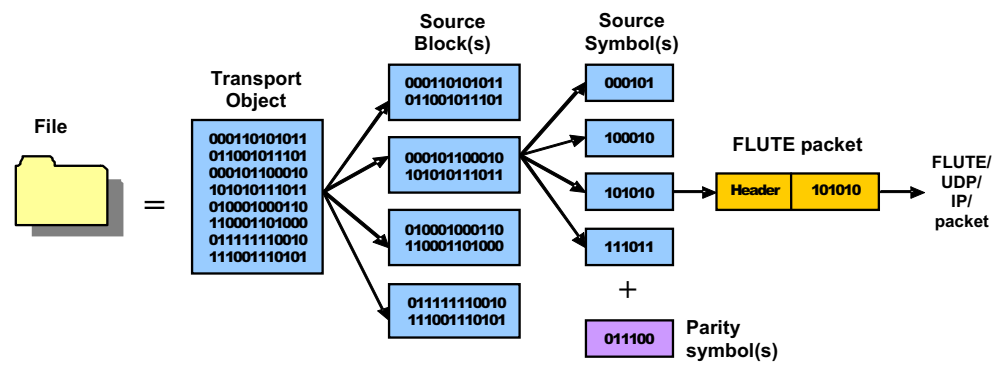

Figure 2.4: FLUTE blocking algorithm.

FLUTE is built on top of the ALC (Asynchronous Layered Coding) protocol instantiation, the base protocol designed for massively scalable multicast distribution [106]. ALC combines the LCT (Layered Coding Transport) building block to provide in-band session management functionality [107], a congestion control building block, and the FEC (Forward Error Correction) building block to provide reliability [108]. The FEC building block allows the choice of an appropriate FEC code to be used within ALC, including the possibility of sending the original data without FEC.

For FLUTE, the file is partitioned in one or several source blocks, as depicted in Figure 2.4. Each source block is split into source symbols of a fixed size. Symbol parameters are signaled in the session setup and are fixed for one session. Then for each source block, FEC encoding can be applied to generate additional repair symbols. The collection of source and repair symbols is generally referred to as encoding symbols.

Each encoding symbol is assigned a unique encoded symbol ID (ESI). If the ESI is smaller than the number of source symbols, then it is a source symbol; otherwise it is a repair symbol. Symbols are either transmitted individually or concatenated and mapped to a FLUTE packet payload. The source block number, the ESI of the first encoded symbol in the packet and other file parameters are signaled in the FLUTE header. FLUTE packets themselves are then encapsulated in UDP and then distributed over IP multicast bearers. Receivers collect correctly received FLUTE packets, and with the information available in the packet header and the file delivery session setup, the structure of the source block can be recovered. Application layer FEC is very beneficial here, as it can be used to provide FEC protection to the file as an entity. An appropriate combination with physical layer FEC can result in very efficient file delivery services.

\subsubsection{Basic Design Considerations}

When designing an upper layer FEC code there are several aspects that should be carefully considered. First of all, if the FEC is not intended to be mandatory for all receivers it should be introduced in a backwards-compatible way for terminals without upper layer FEC decoding capabilities. Hence, the original (legacy) multimedia stream cannot be modified, and a systematic FEC erasure code that keeps the original source packets should be adopted. Its signaling should also be performed in a transparent way for legacy terminals (e.g., using reserved-for-future-use fields in existing standards). 
Regarding the different layers where the upper layer FEC code can be introduced (link or application layer, if the FEC scheme is implemented below or above the IP layer respectively), it should be taken into account the fact that errors propagate in the upper layers of the protocol stack, as the mapping of packet data units at the different layers is in general not aligned. Nevertheless, under a careful design the error correction capability performance should be practically identical regardless of the layer on which the FEC is implemented, and only signaling aspects may differ. In any case, reliable erasure information should be provided at the layer where the upper layer FEC code is to be introduced.

Higher layers also generally imply larger delays. In this sense, LL-FEC may be preferable than AL-FEC for streaming services. However, it should be pointed out that it is necessary to provide the link layer with information regarding the random access points of the multimedia streams.

In principle it is possible to provide service-specific FEC at both link and application layers, although AL-FEC is in general more suitable for providing different protection levels to different streams within a single service. The main benefits of AL-FEC are that it can recover from packet losses of all underlying layers and protocols, providing endto-end error recovery, and that no standardization or modification is required below the application layer. AL-FEC is very beneficial for example for file delivery with FLUTE in IP-based networks, as a single FEC code word can be applied over the entire file, such that a very long time interleaving up to minutes, hours or even days can be applied. In this case AL-FEC is flexible and efficient, as it can be optimized to the service-specific FEC needs.

A careful selection of the maximum time interleaving and code rate of the upper layer FEC code depending on the service requirements and the particular technology under study is essential. In general they will be mainly determined by:

- FEC and interleaving at the physical layer.

- Error characteristic in the system under study.

- Quality of service requirements, in particular delay constraints and target residual error rate.

Finally, when deciding which FEC scheme to adopt, the main aspect to be considered is how close the code performs from an ideal erasure code and the decoding complexity. From a system implementation point of view, the possibility of a full software implementation requiring a small amount of fast memory may be especially beneficial for terminals with limited processing capabilities such as handhelds. Otherwise dedicated hardware will be required at the terminals. A software implementation also eases the introduction of upper layer FEC in already operative systems. Next, we provide performance metrics for UL-FEC codes and review Reed-Solomon and Raptor codes because they are used in UL-FEC mechanisms in DVB-H. 


\subsubsection{Performance Metrics for UL-FEC Codes}

\section{Reception overhead and failure probability}

The reception overhead measures how many encoding packets beyond the minimum possible (i.e., the length of the original source block) are needed to recover the source block by a receiver. In a practical scenario, this would correspond to the number of encoding packets a receiver would request until the decoding is successful. The failure probability is the probability that the source block cannot be decoded by a receiver for a given reception overhead. An ideal code has zero reception overhead and no failure probability. This property is also denoted maximum distance separability (MDS).

\section{Encoding and decoding memory requirements}

The memory requirement for both FEC encoding and decoding processes are metrics of interest, but the decoder memory requirements is the crucial concern, since the amount of memory available that can be used for quick random read/write access can be quite limited in some receiver devices (especially handhelds). The amount of memory needed to encode/decode should be at most approximately the size of the encoded source block, although it is quite beneficial to be able to use less memory for decoding than the size of the source block.

\section{Encoding and decoding speeds}

FEC encoding and decoding speeds are of interest because they increase the end-to-end system delay. The decoding speed is also especially important for handheld devices, because typically the receivers are equipped with low-end CPUs running on batteries, and CPU cycles consume battery power. A representative metric of the encoding/decoding speeds for all FEC codes is the workload, defined as the number of packet XORs used to generate each encoding packet. The workload is often a function of the source block length and the total number of encoding packets generated. Ideally, FEC encoding and decoding speeds should be high enough to ensure that the amount of CPU resources needed is a small fraction of the total available resources.

\section{Sensitivity to packet loss}

Sensitivity to packet loss is when the FEC code performance (reception overhead and failure probability) depends on the loss pattern and the source block size. FEC codes with high sensitivity to losses are not desirable because their performance is rather unpredictable. An ideal code has no sensitivity to packet loss, and it does not matter which specific packets are received but that enough packets are received.

\section{Range of applicable source blocks}

This criterion measures the range of source block sizes for which the FEC code is effective. Ideally, an UL-FEC code should perform well for all applicable source block sizes. 


\subsubsection{Reed-Solomon Codes}

\section{Overview}

Reed-Solomon (RS) codes are a prominent representative of FEC block codes that allow recovering as many lost packets (erasures) as the number of parity packets transmitted. They were discovered in 1960 by Reed and Solomon [109], and can be considered as a special case of a larger class of FEC block codes called BCH (Bose-Chaudhuri-Hochquenghem) codes [110]. However, due to their high encoding and decoding complexity as a function of increasing block length, these codes are commonly only employed in practice for short to moderate block lengths, and RS decoders are typically implemented on hardware. Most Reed-Solomon codes are systematic, meaning that the output codeword contains the input data in its original form.

Reed-Solomon codes operate in general Galois Field (GF) on non-binary symbols and are defined by a generator polynomial. The elements of the Galois field are often referred to as the RS symbols. The block length determines which field the code is defined over. In particular, if $m$ is the number of bits employed to represent each symbol, the block length $n$ is equal to $2^{m}-1$. The most commonly used RS code operates on GF(256) with symbols of eight bits (one byte), such that there is a direct translation between bytes and RS symbols.

Systematic Reed-Solomon codes are usually referred to as $R S(n, k, 2 t)$ code, where the first $k(k \leq n)$ symbols are the source symbols which are to be protected, and the remaining $(n-k)$ symbols are the repair symbols which are calculated based on the source data $(k / n$ being the code rate). The number of parity symbols is usually an even number represented as $2 t$, since a Reed-Solomon code with $2 t$ parity symbols has the capability of correcting up to $t$ errors if the locations of the erroneous symbols are not known, or up to $2 t$ errors if the decoder knows which symbols are erroneous. It does not matter which symbols are received but only that enough symbols are received. On the other hand, when there are too many errors, the RS decoder will not be able to correct anything, and will typically just output the source symbols without error correction.

A RS code operating on symbols of eight bits allows code parameters of any $k<255$, and any $n$, with $k<n \leq 255$. An effectively weaker code than the mother code $k / n$ may be achieved by puncturing, discarding and not transmitting one or more of the last parity symbols. In contrast, a more robust code can be achieved by zero padding the last source symbols, yielding a so-called shortened Reed-Solomon code. These padding symbols are used only for generating the parity symbols but not transmitted. In this case decoders add the removed padding symbols first before decoding. Shortened RS codes provide more robust code rates, but note that the effective block length is reduced.

\section{Code Performance}

RS codes can correct as many lost packets (erasures) as the number of parity packets transmitted if reliable erasure information is provided, having thus zero reception overhead and no failure probability. In this sense they are thus ideal, but only within the tight parameter restrictions for $k$ and $n$. 


\section{Computational Complexity}

RS codes present a very high encoding and decoding complexity, which increases proportionally to the source block size and the proportion of parity data generated. The workload grows as the product of these two quantities. As a consequence only short to moderate block lengths are feasible. RS decoding is prohibitively complex for a software implementation, and hence they are typically implemented on dedicated hardware.

\section{Memory Requirements}

The amount of decoding memory needed for Reed-Solomon codes is proportional to the total size of the encoding packets generated at the transmitter. In general RS decoders at the receiver will reuse the memory required to store the encoded data, such that the additional memory required is low.

\section{Specification}

The code adopted in DVB-H is the $R S(255,191, t=32)$ code with block length 255 bytes, that allows correcting up to 32 random erroneous bytes in a code word of 255 bytes. When reliable erasure information is used, such as provided by the CRC field of the MPE sections, the code allows correcting up to 64 random erroneous bytes.

\subsubsection{Raptor Codes}

\section{Overview}

Raptor codes are a computationally efficient implementation of fountain codes that achieve close to ideal performance [55]. They were invented by Shokrollahi in late 2000 as an extension of LT (Luby Transform) codes [111] with constant encoding and linear decoding $\operatorname{cost}^{2}$. They can be implemented on software without the need of dedicated hardware even in handheld devices, which, in turn, allows supporting large source block sizes. At the receivers, only slightly more data than the original source block is needed for reliable reconstruction compared to an ideal code.

Fountain codes are a special class of FEC codes that can potentially generate an unlimited amount of parity data on the fly (this property is usually coined as rateless). They were originally designed to allow very efficient asynchronous file downloading over broadcast channels without the need of a feedback channel [112]. Figure 2.5 illustrates the concept of an ideal and systematic fountain code, where a transmitter broadcasts the original source packets and a potentially limitless number of repair packets. Receivers tune into the ongoing broadcast session at arbitrary times and leave it once the file is correctly received. The waiting time depends on the experienced channel conditions. No feedback channel is required for retransmissions.

\footnotetext{
${ }^{2}$ Raptor codes are patented by Digital Fountain, recently acquired by Qualcomm, and subject to intellectual property rights.
} 


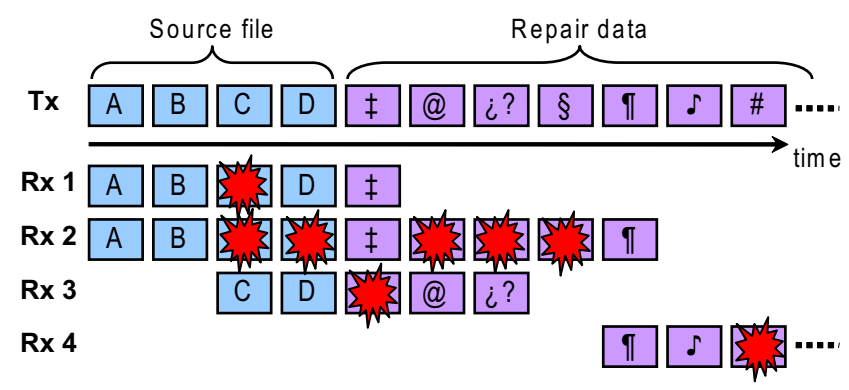

Figure 2.5: Fountain code for asynchronous file download over broadcast channels.

Reliability of this transmission method is provided by the fountain property: as soon as a receiver collects enough packets, it can recreate the source packets (i.e., the original file). This explains the naming "fountain": someone who wants to fill a glass of water under a regular fountain does not care about the particular drops filling the glass; instead, only the amount of water filling the glass matters. Similarly, with a fountain code the particular packets received are not important; only their number matters. Each additional packet is beneficial for reconstruction of the original content and no receiver receives useless information.

The fundamental operational property of a fountain code is that it should be possible to recover the original data with a relative reception overhead - ideally zero, or at least very small - with high probability. Different fountain codes differ in terms of their reception overhead for a given error probability [113], but also in terms of the computational efficiency of the encoding and decoding process. Fountain codes were first mentioned without an explicit construction in [114], and the first efficient construction was invented by Luby [111]. However, LT-codes do not provide constant encoding and linear decoding cost with without sacrificing the error probability.

Compared to LT-codes, Raptor codes achieve their computational superiority using a simple idea: a high rate binary block code is applied before the LT-code. Then the decoder for the LT-code does not need to recover all source symbols but almost all, which is a much easier problem to solve. The drawback is a higher reception overhead for small values of $k$. This can be explained by the fact that for small values the variance of the decoding process is too large, and hence decoding fails more often. Nevertheless, in most practical settings Raptor codes outperform LT-codes in terms of efficiency, range of source block sizes over which it is effective, smaller reception overhead and lower failure probability. A detailed explanation of the coding and decoding processes can be found in [55].

\section{Code Performance}

Raptor codes achieve very close to ideal performance for a wide range of parameters and only slightly more than $k$ symbols are needed to recover the source data independently of the packet loss characteristics (i.e., there is no sensitivity to loss). The reception overhead is a statistical value, and usually a fixed value is considered such that the reconstruction probability is very high. 
The small inefficiency of the Raptor code can be modeled as [77]:

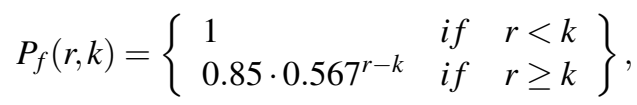

where $P_{f}$ denotes the failure probability of the code with $k$ source symbols if $r$ symbols have been received. While an ideal fountain code would decode with zero failure probability for $r=k$, the failure for Raptor code is still about $85 \%$. However, the failure probability decreases exponentially with an increasing number of received symbols. The increase is so fast, that for only 12 additional symbols the failure probability is $10^{-3}$ and for 24 additional symbols the failure probability is $10^{-6}$. Note that what matters is the absolute number of received symbols, not their fraction compared to the number of source symbols. Thus the reception overhead decreases with increasing $k$. Based on the function $P_{f}(r, k)$, the average reception overhead can be expressed as a function of $k$ as [77]:

$$
\bar{\varepsilon}(k)=\frac{0.85}{(1-0.567) \cdot k},
$$

which can be approximated by $2 / k$. Hence, the number of additional symbols is on average 2 , independent of the value of $k$. Therefore, for typical source block sizes of $k \geq 1000$, the average reception overhead is at most 0.2 percent.

\section{Computational Complexity}

Raptor codes are very attractive in terms of encoding and decoding complexity, as they present a complexity low enough to allow for a full software implementation. Compared to Reed-Solomon codes, the computational complexity of Raptor codes is orders of magnitude less. Furthermore the complexity is linear with the source block size and the rate of parity data generated. Each encoding symbol can be generated on the fly independent of all other encoding symbols, and the average cost of generating each encoding symbol is the same (approximately 7.5 packet XORs). The decoding workload is approximately 10 packet XORs independent of the particular encoding symbols used to reconstruct it [115].

\section{Memory Requirements}

Raptor codes are efficient enough to be applied to directly encode and decode large source blocks. The memory requirement for both encoding and decoding is essentially the source block size, independent of the number of encoding packets sent by the sender or the packet loss characteristics and thus the particular encoding packets received at the receiver. However, it is possible to apply an interleaving technique known as sub-blocking that only requires a small fraction of the source block size in terms of fast memory for decoding with the same performance as if the Raptor code is applied directly on the source block (in terms of reception overhead and failure probability). This approach is very useful when receiver devices contain only a small amount of fast memory that can be used for processing and a much larger store of slow memory that can be used for storing the received data. In these cases, Reed-Solomon codes and direct application of Raptor codes to the entire source block are both impractical, as decoding from the slow memory is not efficient. 




Figure 2.6: Simplified DVB protocol stack including FEC technology.

\section{Specification}

The Raptor code adopted in DVB-H is the same adopted in 3GPP for MBMS [56]. The code is systematic, and it was specifically designed for devices with limited processing and storage resources. This was done by utilizing the fact that for applications for which the code was designed a probability of error of the order of $10^{-4}$ to $10^{-5}$ was acceptable. This led to a design that performs very well for small source block lengths, even if the reception overhead is small. The adopted version can generate up to 65536 encoding symbols onthe-fly from the source data block. It permits coding parameters of $4 \leq k \leq 8192$ and $k \leq n \leq 65546$. A minimum of 1012 source symbols is recommended. For the maximum number of source symbols a code rate of $1 / 8$ can be achieved. Typical maximum source block sizes are 32 and $64 \mathrm{Mb}$, assuming symbol sizes of 512B and $1 \mathrm{kB}$ respectively.

\subsection{Forward Error Correction and Time Interleaving in DVB-H}

DVB-H inherits the physical layer FEC of the DVB-T standard, which is based on a convolutional code concatenated with an outer Reed-Solomon code to correct physical layer MPEG-2 TS packets with only few erroneous bytes. It only provides a time interleaving of up to two OFDM symbols (of a maximum duration $1.12 \mathrm{~ms}$ ) [99].

Regarding upper layer FEC, different solutions are available in DVB in several specifications for different applications [46]. The solutions are partially integrated within streaming and file delivery solutions on the application layer above the IP level; or below the IP level within the link layer MPE (Multi Protocol Encapsulation) and GSE (Generic Stream Encapsulation) protocols. Figure 2.6 provides a simplified protocol stack of different DVB systems and highlights where upper layer FEC is integrated in DVB. In the particular case of DVB-H, three UL-FEC schemes are possible based on Reed-Solomon and Raptor codes. 
Two integrated within the MPE adaptation protocol, that can be used for both streaming and filecasting services: MPE-FEC and MPE-iFEC; and one at the application layer: AL-FEC, but only for file delivery services. Although link and application layer FEC can be applied at the same time, it does not bring any benefit compared to AL-FEC alone, and thus AL-FEC should be employed alone [57].

All schemes are systematic, meaning that the original IP multimedia stream is not modified. In DVB-H each burst consists of an integer number of MPE sections, and each IP packet is encapsulated into a section. With UL-FEC additional parity MPE sections (MPE parity sections) are transmitted to recover from potential IP packet losses. At the receivers each section is considered either completely received or completely lost (erased) based on a cyclic redundancy check (CRC) field. The error correction capability can be then expressed in terms of the maximum number of erroneous MPE sections that can be corrected per source block (assuming the same size for source and parity sections).

\subsubsection{MPE-FEC for DVB-H Streaming Services}

MPE-FEC (Multi Protocol Encapsulation FEC) is an optional intra-burst FEC mechanism at the link layer that was developed during the standardization process of the DVB-H standard. It was designed to compensate for the performance degradations of DVB-T under mobility conditions and to improve the tolerance to impulse interference due to the very short time interleaver employed at the physical layer. The MPE-FEC is typically implemented on hardware, and it basically copes with fast fading in covered areas where static reception is possible, increasing the robustness of reception for mobile terminals such that the signal strength requirement becomes practically independent of the speed [14]. It also increases the maximum speed limit. It provides a similar mobile performance as a DVB-T receiver using two antennas diversity.

The MPE-FEC is specified in [116], and it is based on a Reed-Solomon $\operatorname{RS}(255,191)$ code in conjunction with a block interleaver. It is introduced in such a way that MPE-FEC ignorant (but MPE capable) DVB receivers are able to receive the MPE stream in a fully backwards-compatible way by simply neglecting the additional parity information. The use of MPE-FEC is not mandatory, and is defined separately for each service in the transport stream. For each service it is possible to choose whether to use MPE-FEC or not, and if it is used to choose the trade-off between FEC overhead and transmission robustness.

When MPE-FEC is employed in DVB-H, the IP information is encoded burst by burst with the RS code as shown in Figure 2.7(a). One time-sliced burst carries exactly one MPE-FEC frame. This allows recovering from bursts that are partially received but not from lost bursts. MPE-FEC provides an effective time interleaving depth at the link layer equal to the burst duration (typical values 0.2-0.4 s). The maximum percentage of errors per burst that can be corrected is exactly the proportion of parity data transmitted (e.g., code rate $3 / 4$ can cope with up to $25 \%$ errors). The maximum memory required is about $2 \mathrm{Mb}$ per burst. Since decoding is done on a per-burst basis, the memory requirement is exactly 2 $\mathrm{Mb}$ per stream decoded. Note that several streams may be decoded in parallel. For example when recording one stream while displaying another. Such memory requirements can be supported nowadays by direct on-chip storage. 


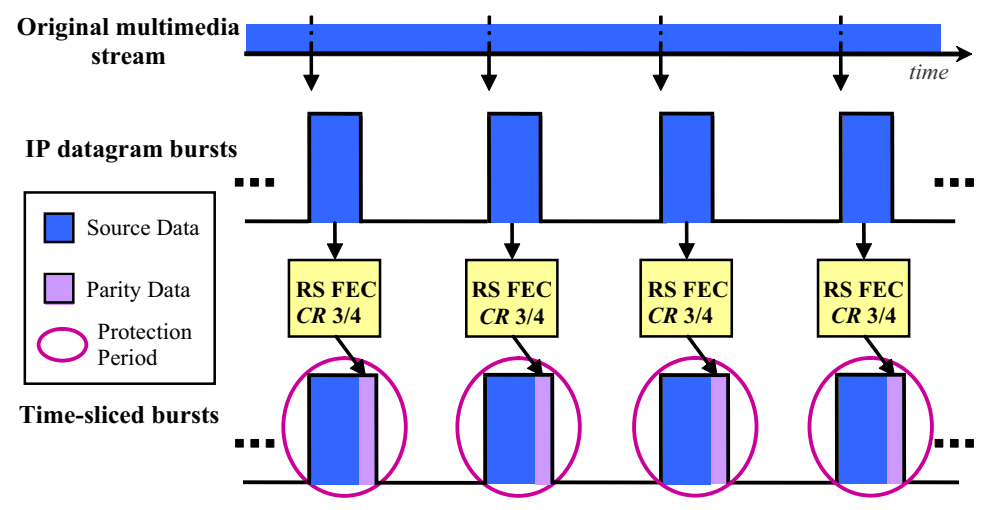

(a) MPE-FEC and time-slicing.

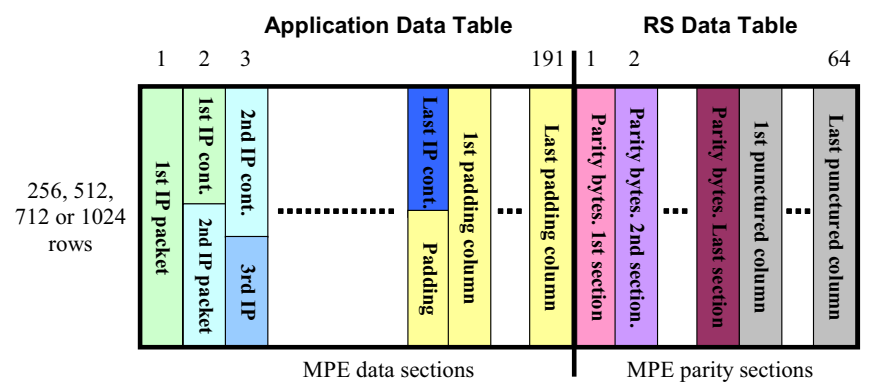

(b) Structure of the MPE-FEC frame.

Figure 2.7: MPE-FEC in DVB-H.

The MPE-FEC frame is structured as a matrix with 255 columns and a flexible number of rows (maximum 1024), see Figure 2.7(b). Each position in the matrix stores an information byte, resulting in a maximum frame size of approximately $2 \mathrm{Mb}$, of which $1.5 \mathrm{Mb}$ are IP data and $0.5 \mathrm{Mb}$ parity data. The frame is structured in two parts: the application data table, dedicated for IP datagrams (191 columns), and the RS data table, dedicated for the parity information (64 columns). Both tables are further structured into MPE sections. Each MPE data section carries one IP datagram, whereas each MPE parity section contains one column of the RS data table.

The process of creating a MPE-FEC frame is the following. First of all, the application data table is filled with IP packets column by column. Once it is filled, any unfilled positions are padded with zero bytes. Next, the RS data table is filled after applying the $R S(255,191)$ code to the application data table row-by-row. Each row of the RS data table thus contains one RS codeword. After coding, IP packets are read out from the application data table and are encapsulated into MPE sections. MPE data sections are followed by the parity data, which is read out from the RS data table column-by-column and is encapsulated into separated MPE sections. The interleaving effect results from the fact that the data is written and read by columns and encoded by rows. 
The parity data is computed with a mother RS code with a code rate $3 / 4$. To allow different code rates, the amount of IP data transmitted per burst can be reduced by padding (shortening) by reducing the number of transmitted columns of the application data table. This yields a more robust code rate. Alternatively, the code rate can be made weaker reducing the amount of parity data transmitted by reducing the number of transmitted columns of the RS data table [14]. It should be noted that different code rates than the mother code $3 / 4$ imply smaller burst sizes. For example, the maximum burst size with a code rate $1 / 2$ is reduced from $2 \mathrm{Mb}$ down to $1 \mathrm{Mb}(0.5 \mathrm{Mb}$ of IP data and $0.5 \mathrm{Mb}$ of parity data). The most common code rates are: $1 / 2,2 / 3,3 / 4,5 / 6$, and $7 / 8$.

Each MPE section has 16 bytes overhead due to the header and a CRC-32 code (to detect erroneous sections). The header contains real-time parameters (e.g., information about the next burst, burst and MPE-FEC configuration parameters), one bit to indicate the last data and parity sections of the frame, and also the start address of its payload in the application data table or the RS data table. For every correctly received section, the receiver will place the payload in its respective table, and mark it as reliable. All correctly received sections (and the padded application data columns if any), are then marked as reliable, whereas all byte positions corresponding to lost sections (and the punctured RS columns if any), are marked as unreliable. If all the data sections within the burst are received correctly, the receiver can neglect the parity sections and switch off until the next burst. Otherwise, if parts of a burst (sections) are lost, the RS decoder is able to correct as many unreliable bytes per row as the number of columns of the RS data table transmitted. If there are more unreliable bytes in a row, the RS decoder will not be able to correct anything and will typically just output the byte errors without error correction. The receiver will have perfect knowledge about the positions of any remaining byte errors within the MPE-FEC frame after RS decoding, and thus only correctly received source IP packets will be available for playback.

It should be pointed out that more sophisticated decoding methods are possible at the cost of higher complexity by working with MPEG-2 TS packets instead of MPE sections (TS packets have a fixed size of 188 bytes, whereas typical sections sizes range between 0.5 and $1.5 \mathrm{kB}$ ) [50]. However the complexity increase is not justified as the potential gain is not significant. In practice TS packet error patterns are heavily correlated such that several consecutive packets are lost, and the loss rate at the link layer is almost the same than at the physical layer.

\subsubsection{AL-FEC for DVB-H File Delivery Services}

DVB-H file delivery with MPE-FEC implies that each of the unique time-sliced bursts where the file is partitioned must be successfully decoded to recover the file. Large files spanning several time-sliced burst will be thus more difficult to deliver, as it will be more likely to lose at least one burst. As users may experience different error patterns, additional error repair can only be achieved by retransmitting the whole file in a carousel, with the problem that terminals will discard the information already received (duplicate received packets are useless). The problem is thus similar to the file download problem in a carousel with repetitions and without FEC described in Section 2.2.3, but at the burst level. 




Figure 2.8: File delivery example in DVB-H using MPE-FEC and AL-FEC. File size is $6 \mathrm{Mb}$ (burst size $2 \mathrm{Mb}$ ). MPE-FEC code rate is $3 / 4$.

In order to increase the robustness of the DVB-H file delivery, an optional FEC mechanism at the application layer (AL-FEC) has been adopted to be used instead of MPE-FEC. With AL-FEC the original source data file is sent uncoded such that it may be interpreted by terminals which do not support Raptor decoding.

AL-FEC is specified in the content delivery protocols (CDP) for file delivery of the IPDC specifications [54], integrated as part of the FLUTE protocol. AL-FEC is optional, but recommended to be used when the file spans more than one time-sliced burst. In practice, with FLUTE files are first partitioned into source blocks, which are encoded and decoded independently. The key with AL-FEC is that it can provide protection across several time-sliced bursts rather than across a single burst as with MPE-FEC. AL-FEC provides almost the same error correction capability as MPE-FEC for a given code rate (Raptor codes operates very closely to an ideal fountain code), but with AL-FEC the effective time interleaving equals to the temporal transmission of the source block. Hence, AL-FEC outperforms MPE-FEC when the source block is transmitted over several bursts. The larger the source block, the higher the gain obtained with AL-FEC compared to MPE-FEC. The gain is twofold: firstly, because filecasting with MPE-FEC performs worse for large files, as the number of bursts that must be correctly received to decode the file increases. This effect happens as well if more robust code rates than the mother code 3/4 are used, and hence reducing the MPE-FEC code rate does not improve performance. Secondly, the larger the source block, the larger the time interleaving with AL-FEC.

As an illustrative example, Figure 2.8 depicts the difference between MPE-FEC and AL-FEC in the delivery of a $6 \mathrm{Mb}$ file [44]. For the sake of simplicity an ideal AL-FEC code has been considered. The code rate assumed for MPE-FEC is $3 / 4$, meaning that the file is divided into 4 bursts (burst size is $2 \mathrm{Mb}$ ), and that can cope with a percentage of erroneous sections per burst up to $25 \%$. With AL-FEC error repair is performed by simply transmitting additional repair data bursts after the original file has been sent. 
With MPE-FEC, if a receiver misses one burst, it must wait until that specific burst is retransmitted, discarding in the mean time data that have already been received. Note also that if one burst is completely received (i.e., all source and parity data), it cannot be used to correct errors in other bursts. On the other hand, with AL-FEC all source and parity data correctly received is useful to the receiver, as it makes no difference which packets are received, accelerating the delivery of the file in such a way that the file reception progresses at approximately the arrival rate of data packets independent of the reception conditions. As a consequence, the time required to deliver files to subscribers is reduced, and more content can be delivered with the same infrastructure [89]. Alternatively, if the transmission time is kept constant, the area coverage for reliable reception is enlarged. The gain can be thus expressed as a link margin gain (coverage extension) or as a reduction of the delivery time (capacity extension).

\subsubsection{MPE-iFEC for DVB-H Streaming Services}

MPE-iFEC [81] was developed during the standardization process of the DVB-SH standard in order to support reception in situations of long signal outages spanning several consecutive time-sliced bursts characteristic of land mobile satellite (LMS) channels. MPE-iFEC defines a generic multi-burst FEC framework which is compatible with the DVB-H link layer. The MPE-iFEC is introduced in such a way that MPE-iFEC ignorant (but MPE and MPE-FEC capable) DVB receivers are able to extract the MPE stream in a fully backwardscompatible way. The source IP packets themselves are sent in MPE sections without any modification compared to MPE-FEC. The use of MPE-iFEC is defined separately for each service in the transport stream. For each service it is possible to choose whether or not MPE-iFEC is used, and if it is used, to choose its configuration. MPE-iFEC extends the MPE-FEC link layer protection of one time-sliced burst to a multi-burst protection using two different approaches:

- One alternative is to extend the interleaver duration with the same Reed-Solomon code employed in MPE-FEC parallelizing the encoding mechanism, such that the source IP data is distributed to a number of parallel encoding matrices and the resulting parity can also be spread over several time-sliced bursts instead of one single burst. This is the sliding Reed-Solomon encoding (SRSE) approach.

- The other alternative consists on encoding several consecutive IP datagram bursts jointly by increasing the encoding matrix size. This requires an encoding algorithm able to efficiently handle very large source blocks. This is the Raptor coding approach.

On the other hand, MPE-iFEC increases the network latency, which is translated into a larger service access time and zapping time between channels, and larger terminal memory requirement, as terminals must wait and store all bursts encoded jointly before decoding and passing the information to the upper layers in order to achieve full protection. However, MPE-iFEC was designed for the purpose of transmitting live video with the goal of minimizing the tune-in delay. 


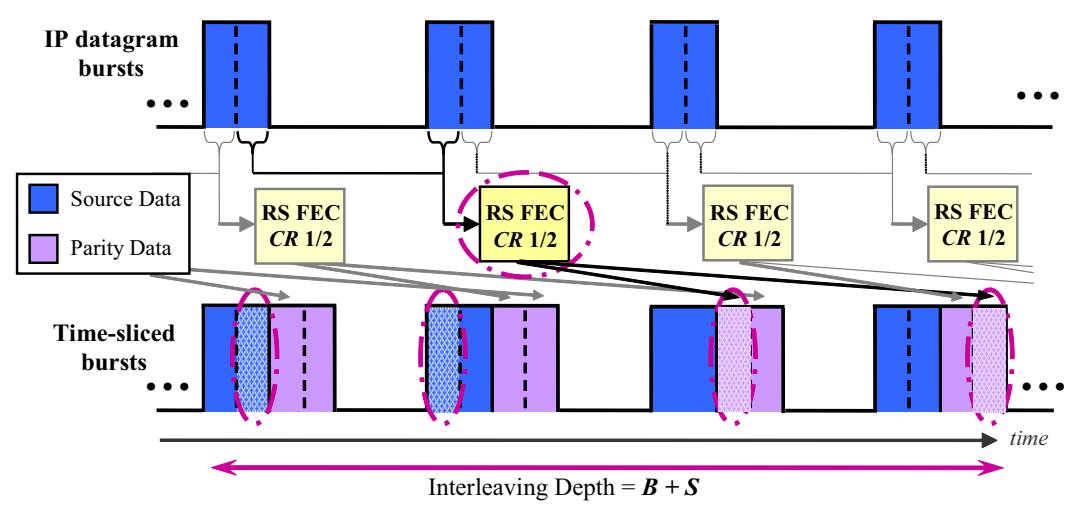

Figure 2.9: MPE-iFEC for DVB-H streaming services with SRSE. Code rate $C R=1 / 2$, encoding parallelization $B=2$, FEC spreading $S=2$, transmission delay $D=0$.

This is done with an information arrangement similar to MPE-FEC, such that each burst contains source IP and FEC parity data, which allows terminals in good reception conditions to directly pass the IP data to the upper layers without MPE-iFEC protection. The MPE-iFEC protection can be recovered later when transmission errors occur. The implications in the network latency and the zapping time are discussed in the next section.

\subsubsection{MPE-iFEC Reed-Solomon Approach}

MPE-iFEC employs the same $R S(255,191)$ code adopted in MPE-FEC, which cannot encode more than one IP datagram burst. In order to provide a multi-burst FEC protection of the transmission a sliding encoding approach is employed. The frequency of the MPE-iFEC coding and decoding process expressed in bursts units is usually referred to as encoding period $(E P)$. In SRSE, it occurs at every burst like in MPE-FEC $(E P=1)$, but a sliding window is used to interleave fractions of several IP datagram bursts into one encoding matrix. Furthermore, another sliding window is used to distribute the FEC parity data from one encoding matrix across several time-sliced bursts. The main parameters that regulate the SRSE operation are the encoding parallelization $(B)$, the FEC spreading $(S)$, and the transmission delay $(D)$ [81]. An example of the operation mode of SRSE is shown in Figure 2.9.

The encoding parallelization is the number of encoding matrices enclosed by the sliding window, and every IP datagram burst is split into $B$ parts distributed over $B$ encoding matrices. The FEC spreading is the number of time-sliced bursts that carry parity data from a single encoding matrix, in such a way that each time-sliced burst carries one IP datagram burst and parity data from $S$ encoding matrices. The transmission delay is the delay applied to the IP information in bursts units. If no delay is applied, the FEC parity data is transmitted in different time-sliced bursts after the IP information, and the interleaving depth in bursts units is equal to $B+S$, as depicted in the figure. Under this setting, terminals must wait and store a total of $B+S$ bursts to retrieve all the IP and parity data to achieve full protection. 
In the figure it can also be observed that up to two lost bursts out of every four can be repaired if the other two bursts are received without errors. However, it may be possible that the same amount of errors cannot be fully repaired if the errors are not uniformly distributed among the encoding matrices. Hence, the actual gain compared to MPE-FEC will depend on the actual distribution of the transmission errors within the interleaved bursts.

By delaying the transmission of the IP datagram bursts it is possible to transmit in the same time-sliced burst IP and parity data corresponding to the same encoding matrix, which effectively reduces the interleaving depth without affecting the values of $B$ and $S$. This happens if $D$ is set to a value between $B$ and $S$. However this configuration degrades the performance of the SRSE mechanism, as the performance improvement compared to MPE-FEC stems from the distribution of the IP and parity data from the encoding matrices over several time-sliced bursts. As a consequence, the best performance is achieved when IP and parity data are transmitted in different time-sliced bursts. This is the case with $D=$ 0 , but also if $D$ is set beyond $B+S$, case where the parity data is transmitted before the IP information. Compared to $D=0, D=B+S$ provides the same performance and introduces the same latency to achieve full protection, but this configuration is more suitable for fast zapping techniques at the expense of a higher end-to-end delay. This is further explained in Section 2.3.5.

Finally, it should be pointed out that the main drawback of adopting SRSE for streaming services in DVB-H is that existing terminals do not count with the necessary hardware to handle the increased memory requirements. Memory requirements are larger because the data interleaving is not limited to one burst but to several ones. The memory requirements are a function of the burst size and the interleaving depth expressed in bursts units. In DVB-SH typical memory requirements per stream are in the order of $12 \mathrm{Mb}$, and they usually require support of host-based storage.

\subsubsection{MPE-iFEC Raptor Approach}

For DVB-H streaming services, Raptor codes may be used to encode several successive IP datagram bursts jointly into one encoding matrix, as shown in Figure 2.10. In this case the encoding period EP takes values higher than one, and the generated parity data is also distributed over EP time-sliced bursts $(B=S=1)$. The normal sending order implies sending the parity data after the IP source information in different time-sliced bursts, since the parity data is computed after all IP datagram bursts are received.

In the figure we can note the similarity between SRSE and Raptor when $B=S=E P$. However although the interleaving depth in burst units is the same, the amount of encoded data interleaved is larger for Raptor coding, due to its capability of coding larger encoding matrices. Thus it potentially provides better performance as it is less vulnerable to the errors time distribution. Indeed, with Raptor coding it is possible to cover all error patterns (combination of lost and partially received bursts) within a protection period as soon as enough packets are correctly received if both IP and parity data are transmitted in the same time-sliced bursts. This configuration provides the best performance with minimal latency, and it is achieved delaying the IP data $E P$ bursts $(D=E P)$, as shown in Figure 2.10(b). 


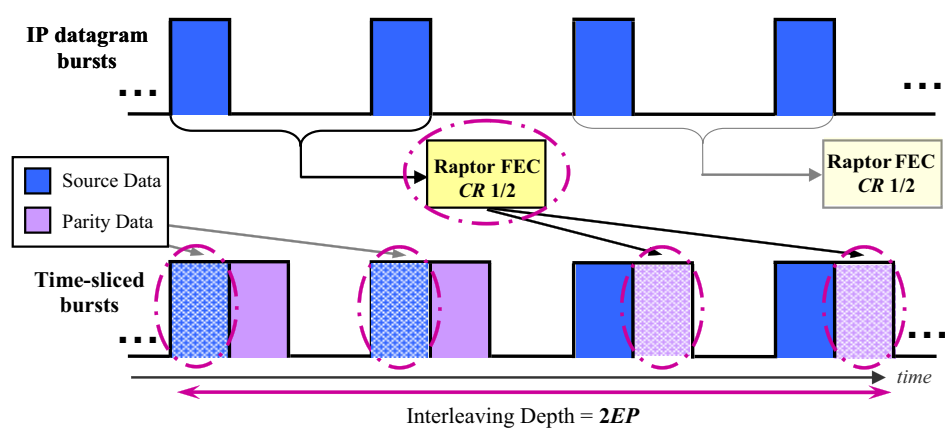

(a) Code rate $C R=1 / 2$, encoding period $E P=2$, transmission delay $D=0$.

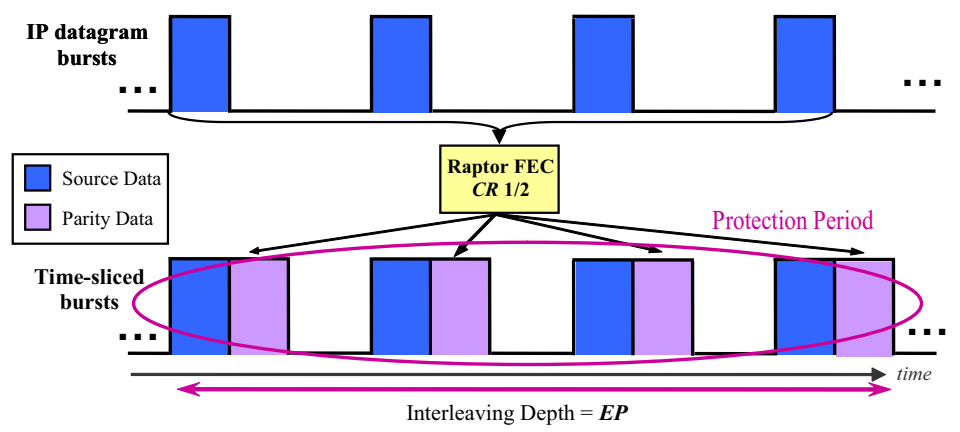

(b) Code rate $C R=1 / 2$, encoding period $E P=4$, transmission delay $D=4$.

Figure 2.10: MPE-iFEC for DVB-H streaming services with Raptor coding.

In this case the interleaving depth equals to $E P$ bursts. However, the configuration most suitable for fast zapping techniques consists on transmitting the parity data before the IP information as with SRSE. This is the case with $D=2 E P$, which keeps the same latency to achieve full protection but increases the end-to-end delay.

The main benefit of Raptor codes for DVB-H streaming services is that they can be implemented in software without the need of dedicated hardware. The multi-burst FEC memory requirement at the terminals is not an issue, and the host memory can be used jointly with the fast memory originally dedicated for MPE-FEC. Another benefit is that the burst size can be chosen freely due to its rateless characteristic, and there is no need to reduce it for different code rates than $3 / 4$ as with SRSE.

\subsubsection{AL-FEC for DVB-H Streaming Services}

AL-FEC is currently not standardized for streaming services in IPDC, but it can be implemented in DVB-H [79], following the FEC streaming framework defined by the IETF and already adopted by $3 \mathrm{G}$ MBMS [56]. Its introduction would require minor changes in the current specifications of the content delivery protocols [54]. 
It should be pointed out that the performance would be practically the same as for MPE-iFEC, and that only some implementation and signaling specific aspects would differ.

\subsubsection{Network Latency and Zapping Time for Streaming Services}

The presence of latency in a unidirectional broadcast system affects the user experience by delaying the initial reproduction of the services, increasing the initial service access time, but also the channel change time between services. For file download services this is usually not an issue. However, for streaming services the zapping time is currently considered as a crucial parameter for mobile TV usability because it has a significant impact on the QoS perceived by the users. Independently whether it is the first access to a service, or the recovery after a signal dropout, it is preferable that the user gets fast feedback about the suitability of the current location for reception. Subjective studies have shown that zapping times of less than 500 milliseconds are perceived as instantaneous, whereas more than two seconds are felt as annoying [101].

The zapping time is defined as the time that a user has to wait to start watching the chosen TV channel since the instant of switching channels. In DVB-H, there is some inevitable delay due to its discontinuous transmission pattern, as users need to wait at least for the first burst of the new channel. The actual zapping time perceived by the users depends on the transmission errors suffered and the time of switching channels, being even possible to receive and display the channel of interest right away. For this reason it is very important to synchronize the multimedia stream with the time-slicing pattern, in such a way that terminals can start decoding the new channel immediately after the first burst has been received if successfully decoded. This can be achieved by including a random access point to the multimedia stream in each burst, and adjusting the buffer size of the codec to the amount of IP source data transmitted per burst. Note that terminals cannot display anything until receiving an Intra-coded frame (I-frame) of the new channel. Only I-frames contain all the information required to decode the complete frame. Most frames are differentially coded and depend on one or more previously transmitted frames (known as $\mathrm{P}$ or B frames). Hence, ideally for DVB-H an integer number of group of pictures (GOP) should be transmitted per burst, as each GOP begins with an I-frame.

With intra-burst MPE-FEC the zapping time in covered areas is fundamentally limited by the time the user has to wait to receive the new burst, being in the worst case a full cycle time, as the processing time required for MPE-FEC decoding can be considered negligible (typical values are about $0.1 \mathrm{~s}$ [117]). The cycle time between bursts, $T_{c}$, depends on the amount of IP source data transmitted in the burst $B_{s}$ (note that it does not include the amount of parity information transmitted in the burst), and the data rate of the multimedia stream $R_{b}$, and it can be computed as:

$$
T_{c}=\frac{B_{s}}{R_{b}}
$$


With multi-burst FEC the zapping time is increased because terminals need to receive several time-sliced bursts with IP and parity data from the same encoding matrix in order to achieve full protection. In this case the zapping time is directly proportional to the interleaving depth, and equals to the number of interleaved time-sliced bursts multiplied by the cycle time (the processing time required by the Raptor decoder can be considered negligible and for SRSE is the same as MPE-FEC).

However, as each burst contains source IP and FEC parity data, terminals in good reception conditions could be able to start reproducing the new TV channel right away as soon as they receive the first burst (if the burst contains a random access point to the multimedia stream as explained before), achieving the same zapping time conditions than with MPE-FEC.

This alternative implies an interruption of the service the first time a burst error occurs ${ }^{3}$, because terminals need to buffer the remaining IP and parity data from the corresponding encoding matrix to achieve full multi-burst FEC protection. If the multi-burst FEC decoding process is successful, the stream will start from the point where it was interrupted. One solution to reduce the buffering time required to achieve full multi-burst FEC protection without reducing the interleaving depth is to delay the IP information at the transmitter such that the FEC parity data is sent ahead of the corresponding source IP data. This is achieved setting the delay parameter $D=B+S$ for SRSE and $D=2 E P$ for Raptor. This implies that the first bursts are not fully protected, as the parity is sent in previous bursts, but it allows a progressive reduction of the buffering time down to the number of bursts with IP data (i.e., $B$ bursts for SRSE, and $E P$ for Raptor).

One solution to hide the buffering time from the perception of the users may be the use of adaptive media playout codecs [118], able to slow down the multimedia stream playout such that the buffer needed for multi-burst FEC decoding can be built over time. Reference [118] suggests that $20 \%$ of speed reduction is hardly perceivable. Such techniques may require modification to existing video decoders, and especially audio decoders.

Fast zapping in DVB-H/SH is a very hot research topic nowadays, and a big research effort is currently being devoted to solve the transition between the fast zapping mode and the regular mode. The actual latency values that will be tolerated by the users implementing such fast zapping techniques are today an open issue. Nevertheless, it is not expected to be able to conceal latencies larger than 10-15 seconds for conventional mobile TV services.

Another simpler alternative to hide the zapping time from the perception of the users is to create a zapping channel with a relatively short cycle time (e.g., 1 second) with complementary information about the different TV channels available [117]. Note that this solution is independent of the FEC scheme employed to protect the TV channels. The aim of the zapping channel is to provide a quick insight about the current content of the TV channels to the users (e.g., snap shots, video and/or sound with reduced quality, etc.). Such information would be updated every burst tracking the content of the associated services.

\footnotetext{
${ }^{3}$ Another issue of the fast zapping mode is that it may lead to a time shift between streams played in different terminals across the service area, as not all of them will experience erroneous bursts at the same time. With maybe few exceptions (e.g., real-time transmissions of sport events), this shift may be well tolerated by the users, as the channels with non-real-time content will probably continue being dominant [6].
} 


\subsection{AL-FEC in Hybrid Cellular and DVB-H Systems}

Among the different FEC mechanisms adopted in DVB-H, application layer FEC with Raptor coding is particularly well suited for hybrid cellular and DVB-H systems [38]. Specific benefits of working at the application layer are:

- No standardization or modification is required below the application layer, as it operates transparently above the IP layer.

- It can recover from packet losses of all underlying layers and protocols, providing end-to-end error recovery (e.g., it can even recover IP packets lost in the core network or the Internet).

But is the use of Raptor coding which makes AL-FEC outperform other FEC solutions in terms of implementation complexity, flexibility, and efficiency. An important consideration is system integration, and they allow for a software implementation. Another important benefit is the capability of encoding large amounts of source data (e.g., $64 \mathrm{Mb}$ ). Moreover, thanks to their rateless capability it is possible to produce an enormous amount of parity data (the standardized version supports, at least, a code rate 1/8). This is the actual property which enables efficient and flexible error correction in mobile broadcasting systems, because there is no need of retransmitting specific source packets. Error correction is achieved transmitting additional parity packets that can be used by all users once the original source information is transmitted.

This property also enables efficient point-to-multipoint repair transmissions in hybrid unicast-broadcast systems in the situations where too many users fail to receive the initial transmission. Another important benefit of Raptor coding is that it allows generating additional repair packets on-demand, without knowing the total number of repair packets needed. Moreover, terminals do not need to notify the repair server the specific packets they need to avoid duplicate packet reception.

In a hybrid evolved 3G+ and DVB-H system, AL-FEC with Raptor coding enables very efficient error repair, because from the users' point of view it does not matter whether parity data is received though unicast with HSDPA, multicast with MBMS, or broadcast with DVB-H. Furthermore, the repair data transmitted through a cellular network may be generated by a different server. 


\subsection{Conclusions}

In this chapter we have reviewed in detail upper layer FEC in DVB-H, describing the different mechanisms available at the link and application layers. They aim to enlarge the time interleaving of the information, which at the physical layer is only in the order of a couple of milliseconds, to take advantage of the time diversity of the mobile channel.

The conventional intra-burst MPE-FEC scheme developed during the standardization process of DVB-H provides a time interleaving equal to the burst duration (typical values 0.2-0.4 s), which is only efficient to recover from partially received bursts but not from completely erroneous bursts. Multi-burst FEC schemes such as MPE-iFEC and AL-FEC can provide protection across several bursts rather than within a single burst as MPE-FEC. This way it is possible to correct not only partially received bursts, but also lost bursts. The time interleaving depends on the number of bursts jointly encoded and the cycle time between bursts. The drawbacks are that the network latency and the terminal memory requirement are increased, because terminals must wait and store several bursts before decoding.

Multi-burst FEC is currently only standardized in DVB-H for file delivery services as AL-FEC with Raptor coding. The key advantages of Raptor codes are that they can encode large amounts of data, and that they can be implemented in software without the need of additional hardware. The multi-burst FEC memory requirement at the terminals is not an issue, and the host memory can be used jointly with the fast memory originally dedicated for MPE-FEC. As a consequence, they can be introduced as a software update, being backwards-compatible with existing networks and terminals. But Raptor codes can be applied to streaming services in DVB-H as well. This can be done either at the application layer or at the link layer, achieving practically the same performance. Only some implementation and signaling specific aspects differ.

File download services will be able to fully exploit the benefits of multi-burst FEC, but for streaming services the latency is translated into a larger initial access time and zapping time between channels, which are crucial parameters for mobile TV usability. Therefore, only specific streaming services able to tolerate large latencies (e.g., some video clips, specific news channel for vehicles in urban areas), could fully exploit the gains obtained by performing a multi-burst protection of the transmission. For conventional mobile TV services, it is necessary to implement so-called fast zapping techniques to conceal the multi-burst FEC latency from the perception of the users. The actual latency values that will be tolerated by the users implementing such fast zapping techniques are today an open issue, but it is not expected to be able to conceal more than 10-15 seconds. Therefore, the gain will be limited.

Finally, we have explained the benefits of using AL-FEC in a hybrid cellular and DVB-H system to enable an easy and efficient interworking of the repair mechanisms. With AL-FEC repair data can be efficiently and seamlessly delivered through p-t-p unicast transmissions or p-t-m multicast/broadcast transmissions. 


\section{Chapter 3}

\section{Multi-Burst Forward Error Correction in DVB-H}

In the previous chapter, we have seen that Raptor coding can be introduced in existing DVB-H networks and terminals as a software update (either at the link layer or the application layer) to encode several bursts jointly. Compared to the conventional intra-burst MPE-FEC mechanism, this multi-burst FEC scheme can improve the transmission robustness for streaming services by delivering the content as a succession of larger source blocks spanning several bursts ${ }^{1}$ [79]. For file download services it can provide protection across the whole file rather than a single burst [89]. In this chapter we evaluate the potential gain that can be obtained with such multi-burst protection of the transmission. Both MPE-FEC and multi-burst FEC schemes aim to correct erroneous MPEG-2 TS packets at the physical layer.

\section{Physical Layer Transmission Errors in DVB-H}

Generally speaking, mobile users experience two types of errors at the physical layer when moving around a DVB-H network with imperfect coverage of the service area: completely erroneous (lost) bursts and partially received bursts. Of these, lost bursts have a major impact on the service coverage because they are perceived as a coverage discontinuity. This situation may be especially evident in the initial phases of the DVB-H network deployment, where mobile users experience temporary lack of coverage (outage areas) when moving across the service area. But mobile users may experience errors in covered areas as well because of the vulnerability of the physical layer to signal fading (fast fading and shadowing) and impulse noise.

\footnotetext{
${ }^{1}$ For streaming services it possible to provide a multi-burst protection of the transmission with the same Reed-Solomon code adopted in MPE-FEC employing a sliding window approach. However, existing DVB-H terminals do not count with the necessary hardware to handle the increased memory requirements. Performance evaluation results of sliding Reed-Solomon encoding (SRSE) for DVB-H streaming services can be found in [82].
} 


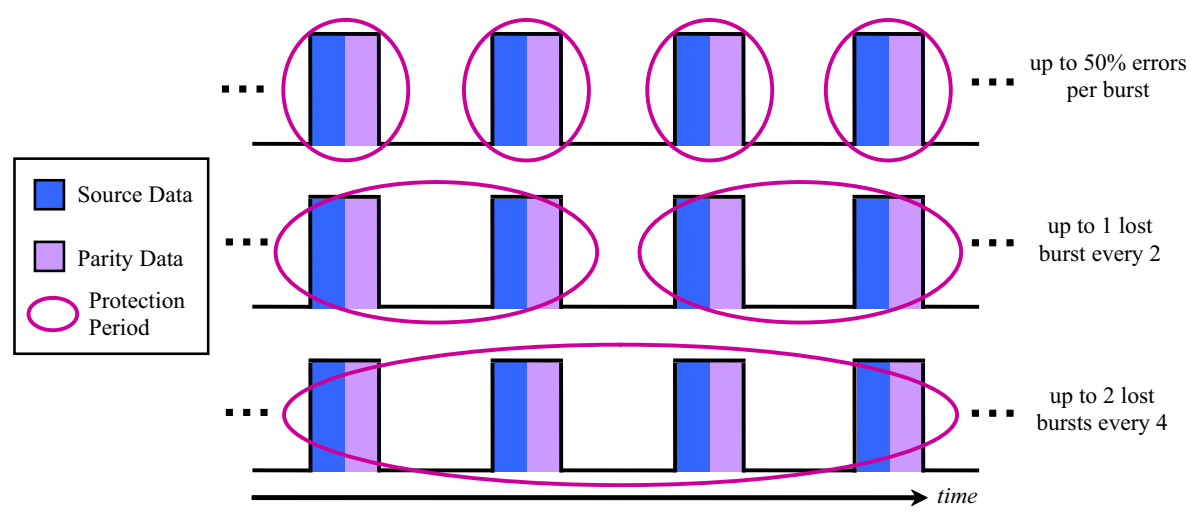

Figure 3.1: Multi-burst FEC concept in DVB-H and error correction capability with $1 / 2$ code rate.

In particular, terminals can miss an entire burst completely due to temporary shadowing. This occurs due to the very rapid transition from near perfect reception to no reception at all behavior or the underlying DVB-T standard with the received signal strength. However, this fast transition only applies for static reception conditions. The reason is that DVB-T was primary designed for fixed rooftop reception with a very short time interleaving, being very vulnerable to fast fading in mobile channels and impulse noise. As a consequence, under mobility conditions the performance degradation with fast fading enlarges the dynamic range up to 10 to $15 \mathrm{~dB}$ [52]. This implies that in covered areas mobile users may also receive only a part of a burst due to fast fading. With MPE-FEC it is possible to recover from bursts partially received, but not from lost bursts [14]. In order to recover from these errors a FEC scheme spanning multiple bursts is required [44].

\section{Multi-Burst Encoding Concept}

Figure 3.1 shows three different ways of transmitting the same content using intra-burst FEC and multi-burst FEC with the same code rate $1 / 2$ but different protection periods. It should be noted that each time-sliced burst contains both source IP information and FEC parity data. The IP data is obviously the same in the three cases, but not the parity data; which is computed across different IP datagram bursts. We will use the figure to illustrate the coding efficiency improvement that is achieved by encoding more bursts jointly. Although the parity data is the same in the three cases, the level of protection is different.

For the sake of simplicity and clarity, let's assume an ideal code, such that the maximum percentage of errors that can be corrected is equal to the proportion of parity data transmitted. Then, for the intra-burst FEC case, if the percentage of errors in a burst exceeds 50\% the FEC decoder will fail and only correctly received IP source packets will be available to the applications. For the cases with multi-burst FEC it can be corrected the same percentage of errors, $50 \%$, but across several bursts; being possible to compensate with up to one and two completely erroneous bursts if all other bursts within the protection period are received without any errors. 
Multi-burst FEC can be seen as a technique to benefit from the time diversity of the mobile channel. Intuitively, the lower the statistical correlation between bursts reception conditions, the higher the gain. For fully static reception conditions there is no gain, as users will either receive all content or nothing. On the other side, mobile users experience variations in their reception conditions when moving across the service area, but also due to dynamic variations in the surroundings of the receiving environment. The gain will thus increase for higher user velocities, and for this reason multi-burst FEC can be expected to be especially suited for vehicular users. Lower gains can be expected for pedestrian users due to their reduced mobility.

In any case, the larger the number of bursts jointly encoded, the higher the interleaving and the diversity gain, enhancing the coding efficiency to protect against temporary errors. The improvement is especially evident for robust code rates where a significant amount of parity data is transmitted. For example, with a code rate $7 / 8$ only one lost burst out of eight can be recovered. However, it should be pointed out that if the code rate is not robust enough to correct the total number of errors there may be no gain by increasing the interleaving, and the performance may even degrade. Increasing the interleaving pays off when the total amount of errors is smaller than the error correction capability.

\section{Performance Evaluation}

The gain of multi-burst FEC compared to MPE-FEC will depend on several factors, such as the code rate, the interleaving depth in number of bursts jointly encoded, the FEC scheme employed and the target residual error rate. However, the actual gain is very difficult to quantify in real life, as it depends on the time evolution of the transmission errors experienced by the users, and hence on the velocity and trajectory of the users. One important parameter is for example the coverage level perceived by the users. As if it is too high there won't be any error to correct, whereas if it is too low it won't be possible to recover any data. The gain will thus not be constant across the service area, and it cannot be directly included in the link budget [84]. The problem of estimating the coverage for multi-burst FEC DVB-H services is addressed in Chapter 4. In this chapter we evaluate the performance of multi-burst FEC with field measurements, and quantify the reduction in the carrier-to-noise ratio (CNR) requirement to cope with shadowing and fast fading (fading margin gain). This gain can be easily interpreted in terms of network planning, as it can be directly translated into a coverage gain.

As mentioned before, MPE-FEC was mainly introduced to cope with fast fading. It increases the robustness of reception for mobile terminals such that the signal strength requirement becomes practically independent of the speed. Its effect is taken into account in the receiver sensitivity (minimum signal level at which reception is possible) [14]. On the other hand, to cope with shadowing a fading margin of several decibels is added to the link budget in order to assure a certain high location probability. When considering this location variation the values are generally considered to follow a log-normal distribution. The standard deviation value commonly used with broadcasting signals in the UHF band is $5.5 \mathrm{~dB}$. Typical location probability values recommended for DVB-H are $95 \%$ for mobile and portable reception, and $99 \%$ for vehicular reception [34]. 

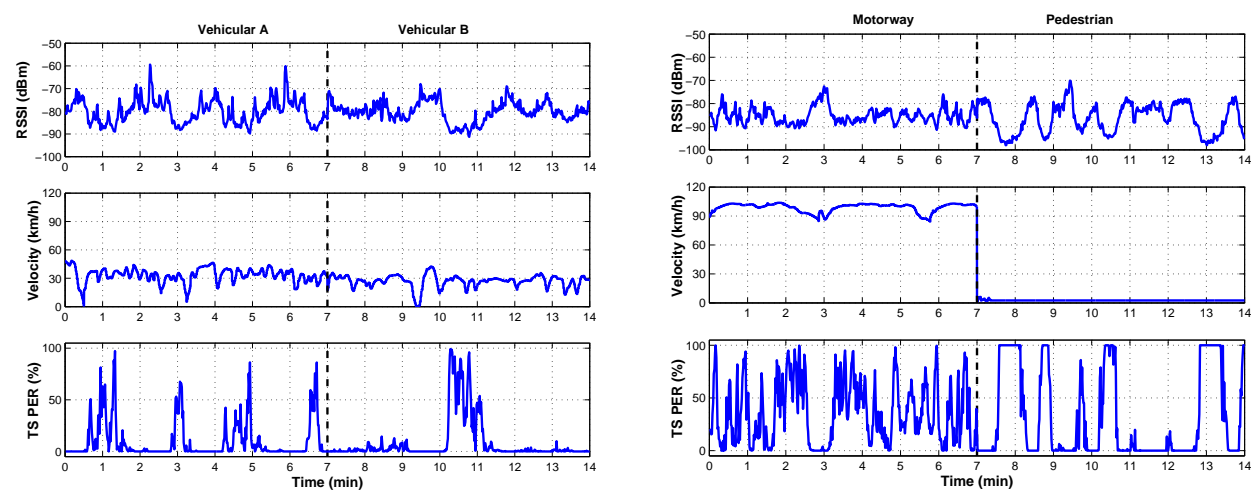

Figure 3.2: Example data of DVB-H field measurements for vehicular reception conditions (left) and for motorway and pedestrian indoor reception conditions (right).

\begin{tabular}{|c||c|c|c||c|}
\hline \multirow{2}{*}{$\begin{array}{c}\text { Trace ID and } \\
\text { reception case }\end{array}$} & \multicolumn{3}{c||}{ MPEG-2 TS } & \multirow{2}{*}{$\begin{array}{c}\text { MPE section } \\
\text { error rate }\end{array}$} \\
\cline { 2 - 4 } & PER & AEBL & VEBL & $11.2 \%$ \\
\hline \hline A - Vehicular & $10.5 \%$ & 28.1 & $1.7 \cdot 10^{4}$ & $10.7 \%$ \\
\hline B - Vehicular & $10.1 \%$ & 40.0 & $4.0 \cdot 10^{4}$ & $39.5 \%$ \\
\hline C - Motorway & $37.6 \%$ & 41.1 & $3.2 \cdot 10^{4}$ & $38.0 \%$ \\
\hline D - Ped. Indoor & $37.5 \%$ & 176.6 & $10.6 \cdot 10^{7}$ & \\
\hline
\end{tabular}

Table 3.1: Measured packet error statistics.

To account for a practical implementation of a multi-burst FEC code, a constant $1 \%$ reception overhead has been assumed. In the case of Raptor coding, this will generally allows recovery of the original content in most of the cases [57], see Chapter 2.2.7.

\subsection{Field Measurements Results}

Field measurements were performed for vehicular and pedestrian reception conditions in the DVB-H pilot networks of the universities of Turku (Finland) and Braunschweig (Germany). Both cities can be considered sub-urban environments for European standards. The measurement set-up and the measurements performed are described in Appendix A. Measurements consisted of MPEG-2 TS packet error information at the physical layer of the whole multiplex. Results are obtained emulating the upper layers in software (time slicing, protocol decapsulation, and FEC mechanisms). To achieve a statistical smoothing of the results, results have been averaged within the cycle time with a burst duration resolution.

Figure 3.2 depicts four traces measured in Turku that will be used as representative examples of the different reception conditions. Table 3.1 shows the packet error statistics, including the MPEG-2 TS packet error rate, average error burst length (AEBL) and its corresponding variance (VEBL), and the MPE section error rate (IP packet size 512 bytes). 

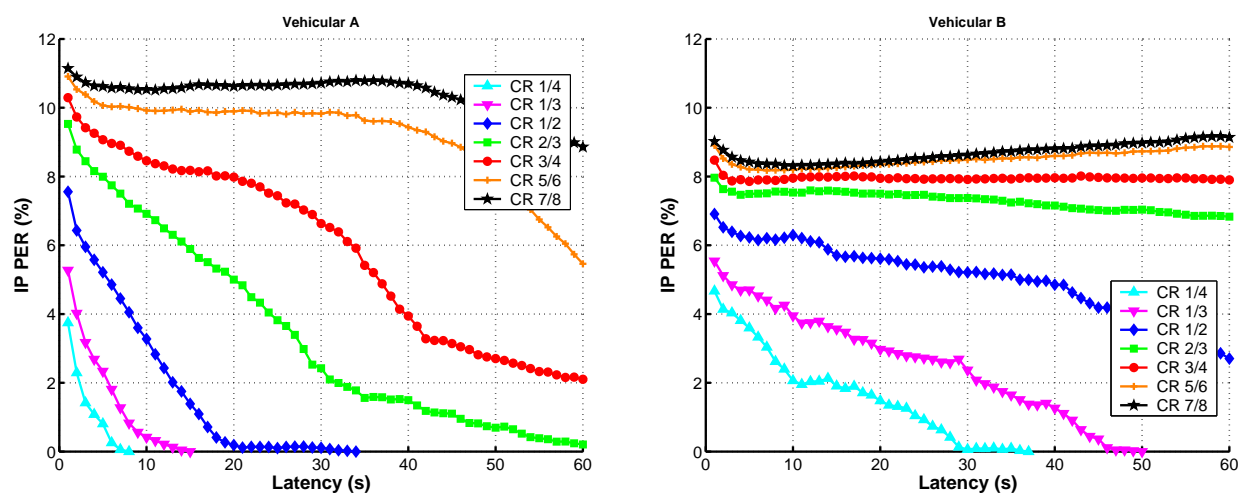

Figure 3.3: IP packet error rate of a streaming service at $384 \mathrm{~kb} / \mathrm{s}$ vs. Latency for the vehicular traces shown in Figure 3.2.

For streaming services we employ the IP packet error ratio (IP PER) of the streaming service (percentage of erroneously received IP packets) as the performance measure to compare the robustness of different FEC schemes for a given measured trace. Although it only accounts for the overall transmissions errors, it allows an easy, objective and fair comparison between MPE-FEC and multi-burst FEC. For file download services we provide some illustrative results of the performance behavior of MPE-FEC and multi-burst FEC for file delivery as a function of the different transmission parameters: file size, code rate, cycle time between bursts and burst size. The performance measure employed is the probability of receiving successfully the file across the measured trajectory (file acquisition probability).

\subsubsection{Streaming Results - Vehicular}

Figure 3.3 shows the percentage of erroneously received IP packets of a $384 \mathrm{~kb} / \mathrm{s}$ streaming service as a function of the network latency for different multi-burst FEC code rates for the vehicular measured traces shown in Figure 3.2 [79]. In the figure the markers shown correspond to an entire number of bursts coded jointly. The first marker to the left is the reference intra-burst FEC case. We assume a constant IP packet size equal to 512 bytes and a constant cycle time between bursts of 1 second (i.e., the burst size is variable and depends on the code rate). This way the latency is equal to the number of bursts jointly encoded in seconds and the maximum zapping time with intra-burst FEC is 1 second. In the figures an IP PER value equal to zero means that all streaming content is received correctly without errors. We can notice very different slopes for each trace despite of having a similar amount of errors (MPEG-2 TS PER and MPE section error rate, see Table 3.1). Indeed, for trace $B$ there is no gain increasing the interleaving for high code rates. This is due to the different distribution of the transmission errors over time. As trace $A$ presents a more uniform error distribution pattern, shorter interleaving (latencies) are necessary. 


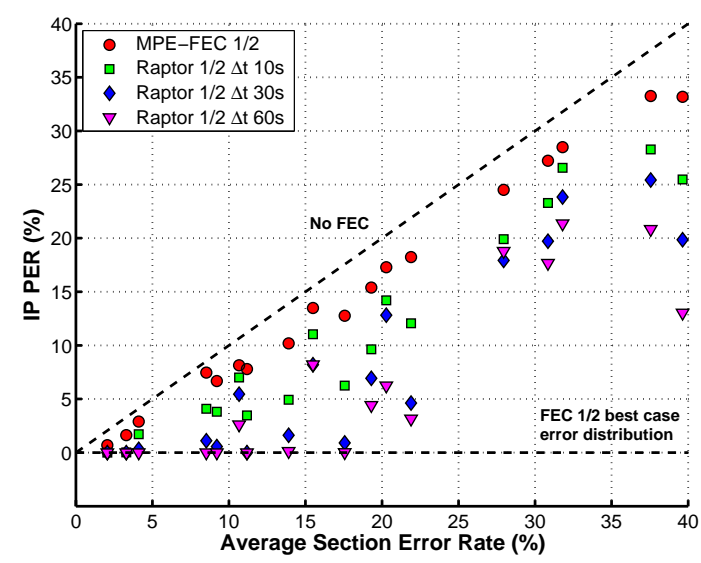

Figure 3.4: IP packet error rate of a streaming service at $384 \mathrm{~kb} / \mathrm{s}$ vs. Average MPE section error rate for all vehicular trajectories measured in Turku.

In Figure 3.3 we can see that for trace $B$ most errors are concentrated within one minute, whereas for trace $A$ we can distinguish four error bursts distributed over time. This is also supported by the higher AEBL and VEBL values for trace $B$ shown in Table 3.1, which usually denote a more concentrated error distribution for the same amount of errors.

Figure 3.4 compares the IP PER achieved with MPE-FEC and multi-burst FEC for different latencies (code rate 1/2) as a function of the average MPE section error rate for all the vehicular trajectories measured in Turku. The total measurement time was over 2 hours, divided into 18 measurements of 7 minutes each. We also depict the reference case without FEC at the link and application layer, and the maximum error correction capability for a code rate $1 / 2$. Ideally, if the errors are uniformly distributed it is possible to cope with error rates equal to the proportion of parity data transmitted (e.g., code rate $1 / 2$ can cope with up to $50 \%$ erroneous MPE sections). However, in practice the DVB-H channel is very bursty, as we can see in Figure 3.2, and long error bursts are common. As a consequence, the coding efficiency is reduced, being not able to correct all errors. This degradation can be solved with multi-burst FEC increasing the time interleaving by coding more bursts jointly. If a sufficiently large time interleaving is employed, it is possible to cope with as many errors as in the best case.

In the figure we can see that the performance of MPE-FEC is relatively close to the case without any FEC at all, and that it can only compensate small error rates to provide good service quality (up to 5\% MPE section error rates for IP PER values of 5\% or lower). As the interleaving depth equals to the burst duration (about $150 \mathrm{~ms}$ in this example), it cannot cope with longer signal outages. Hence, MPE-FEC will be only effective in very dense SFN networks with very good coverage level over the service area to compensate for the fast fading due to the mobility of the terminals. With multi-burst FEC the interleaving depth takes values of several seconds, but the gain varies from trace to trace depending on the error distribution. Nevertheless, we can note interesting gains with only 10 seconds latency, being able to cope with up to $10 \%-15 \%$ MPE section error rates. 


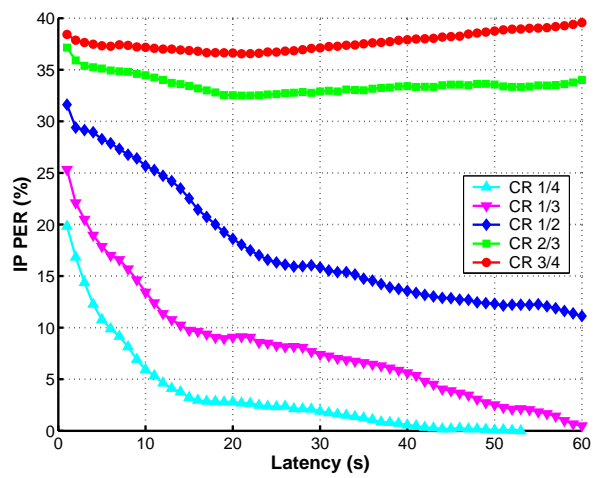

(a) Motorway

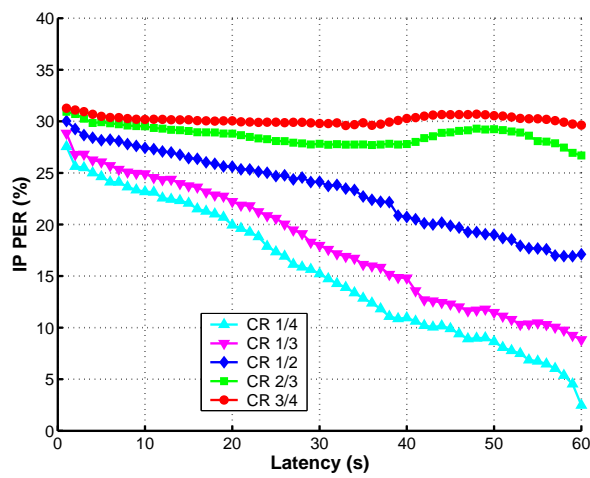

(b) Pedestrian

Figure 3.5: IP packet error rate of a streaming service at $384 \mathrm{~kb} / \mathrm{s}$ vs. Latency for the motorway and pedestrian traces shown in Fig. 3.2.

\subsubsection{Streaming Results - Motorway and Pedestrian}

Figure 3.5 shows the IP PER of a $384 \mathrm{~kb} / \mathrm{s}$ streaming service as a function of the network latency for different multi-burst FEC code rates for the vehicular motorway and pedestrian indoor traces shown in Figure 3.3. These traces have considerably more errors than the vehicular traces considered in the previous section (almost $40 \%$ erroneous MPE sections), and hence a saturation effect for high code rates that cannot cope with so many errors occurs (code rates $2 / 3$ and $3 / 4$ ). For more robust code rates the improvement is clear when increasing the latency. The IP PER reduction is very fast for the motorway trace, whereas for the pedestrian trace is noticeably slower.

The gain obtained for pedestrian users is smaller due to their reduced mobility. In Figure 3.2 outage periods can be observed in the pedestrian trace where all packets received are erroneous, which imply that longer interleaving are required (see also very large AEBL and VEBL values in Table 3.1). On the other hand, in the motorway trace the errors appear almost across the whole trace, being much more distributed. Errors at the physical layer are common for high velocities due to fast fading impairments because of the very short time interleaving employed.

We can also notice the completely different error distribution patterns looking at the intra-burst FEC case in Figure 3.5. For the pedestrian trace we can see that the IP PER reduction between the weakest and the most robust code rates considered is very small, whereas for the motorway trace the reduction is significant. These results agree with the performance behavior of MPE-FEC, providing its maximum gain for high Doppler frequencies (vehicular reception) and its minimum gain for low Doppler frequencies (pedestrian reception), being indeed no needed at all as it is preferable to employ a more robust transmission mode at the physical layer instead of using MPE-FEC [119]. 


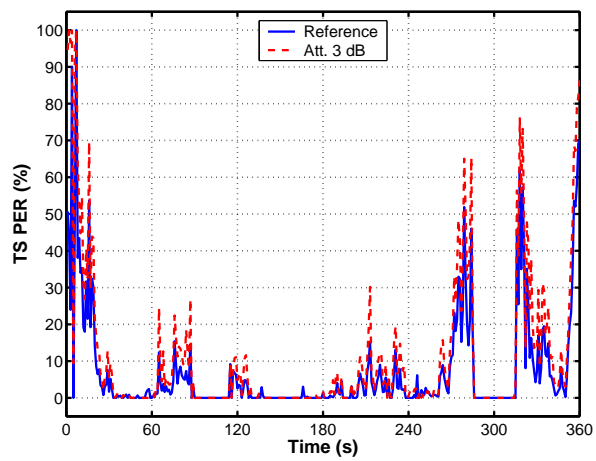

(a) Attenuation $3 \mathrm{~dB}$.

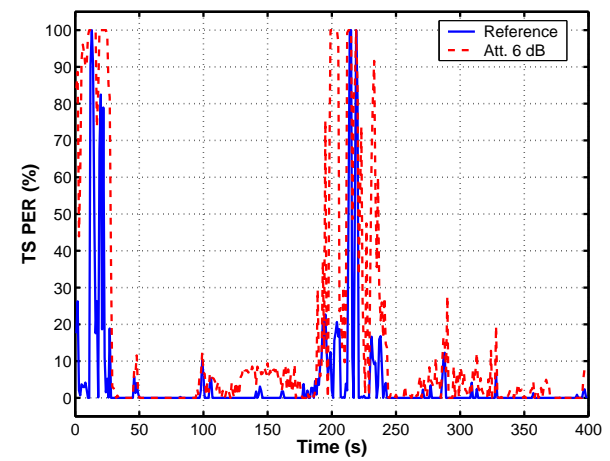

(b) Attenuation $6 \mathrm{~dB}$.

Figure 3.6: Vehicular DVB-H field measurements with two receivers, one attenuated with respect to the other. Measured TS packet error rates.

\subsubsection{Streaming Results - Link Margin Gain Example}

To provide further insight into the potential gain that can be achieved with multi-burst FEC for streaming services in real scenarios, field measurements were performed in Braunschweig for vehicular reception conditions with two receivers with a common antenna but one receiver attenuated compared to the other. This enables a comparison of the two signals with a constant offset in the received power. Figure 3.6 shows two examples of the MPEG-2 TS packet error rate per second recorded by the two receivers with an attenuation of $3 \mathrm{~dB}$ and $6 \mathrm{~dB}$. The solid line represents the reference receiver and the dashed line represents the attenuated receiver.

Figure 3.7 shows the IP packet error rate of a $384 \mathrm{~kb} / \mathrm{s}$ streaming service for different multi-burst FEC code rates as a function of the latency for both measurements. In the figure it can be seen that for the reference signals there are few errors and the IP PER is around 5\% for the intra-burst FEC case. Increasing the latency encoding more bursts jointly reduces the IP PER improving the quality of the received service. For the attenuated signals larger latencies are necessary in order to achieve similar residual error rates than the reference signals with intra-burst FEC, especially for the case with an attenuation of 6 $\mathrm{dB}$. In Figure 3.7(a) we can see that a $3 \mathrm{~dB}$ link margin gain is achieved for latencies lower than $10 \mathrm{~s}$. In Figure 3.7(b) we can see that a $6 \mathrm{~dB}$ link margin gain is achieved for latencies of 23 and $44 \mathrm{~s}$ respectively, whereas it is not possible to cope with the $6 \mathrm{~dB}$ attenuation with code rates $2 / 3$ and 3/4 for latencies lower than $60 \mathrm{~s}$. 




(a) Attenuation $3 \mathrm{~dB}$.

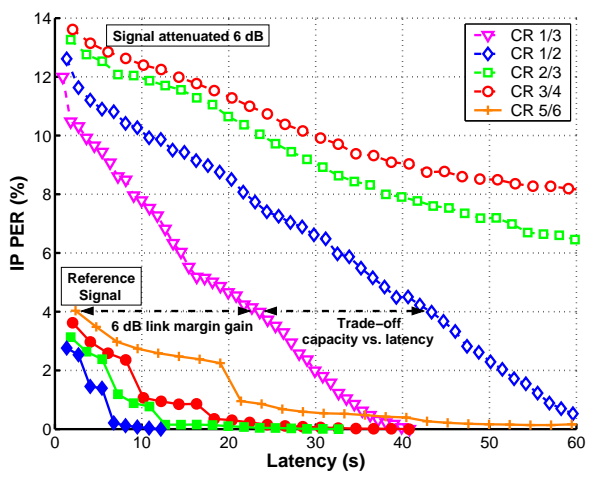

(b) Attenuation $6 \mathrm{~dB}$.

Figure 3.7: Vehicular DVB-H field measurements with two receivers, one attenuated with respect to the other. IP packet error rate of a $384 \mathrm{~kb} / \mathrm{s}$ streaming service vs. latency.

\subsubsection{File Download Results - Vehicular}

In this section we present illustrative results comparing the performance of MPE-FEC and multi-burst FEC with Raptor coding for file delivery for the vehicular field measurements shown in Figure 3.2. The performance measure is the probability of receiving the file across the measured traces.

Figure 3.8(a) shows the file acquisition probability as a function of the file size. Transmission parameters considered are: burst size $1 \mathrm{Mb}$, cycle time between bursts $2 \mathrm{~s}$ (constant download data rate $500 \mathrm{~kb} / \mathrm{s}$ ), and code rate 3/4 for both MPE-FEC and multi-burst FEC. In the figure we can see how the acquisition probability decreases with the file size for MPE-FEC whereas it increases for multi-burst FEC. With MPE-FEC, the larger the file, the higher the number of bursts into which the file is partitioned; and since all of them must be correctly received it becomes more difficult to deliver larger files as it is more likely to lose at least one burst. In contrast, with multi-burst FEC larger files imply longer interleaving, and hence the FEC coding efficiency is increased.

Figure 3.8(b) shows the acquisition probability of a $6 \mathrm{Mb}$ file as a function of the code rate. The transmission parameters previously used are kept. For MPE-FEC the burst size is adjusted according to the code rate (burst size is $1 \mathrm{Mb}$ for code rate 3/4). It can be observed that the acquisition probability for multi-burst FEC is directly proportional to the code rate, but not for MPE-FEC. The problem of MPE-FEC for file delivery is that more robust code rates than 3/4 imply smaller burst sizes ${ }^{2}$, which increases the number of bursts that must be successfully received and degrades the performance. Hence, it can be considered the code rate $3 / 4$ to be optimum.

\footnotetext{
${ }^{2}$ For example, the maximum IP information that can be transmitted per burst for a code rate $1 / 2$ is reduced from 1.5 Mb to 0.5 Mb, see Chapter 2.3.1.
} 


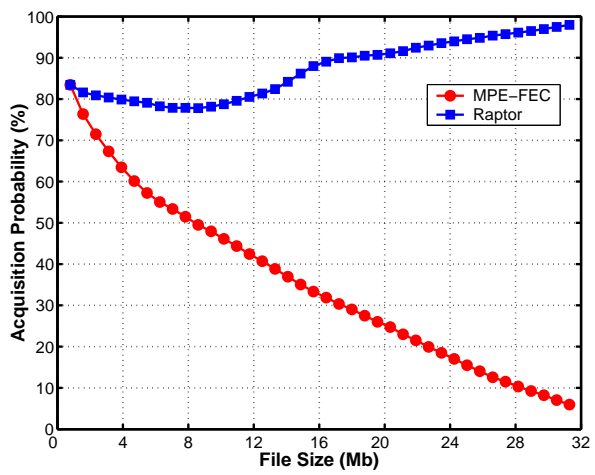

(a) $C R=3 / 4, B_{S}=1 \mathrm{Mb}, T_{c}=2 \mathrm{~s}$.

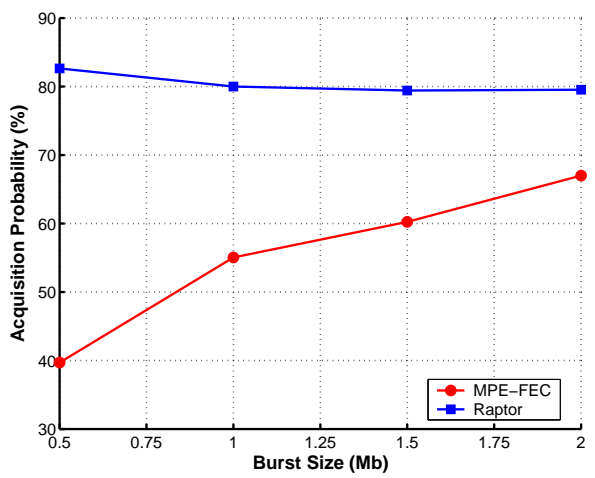

(c) $F_{s}=6 \mathrm{Mb}, C R=3 / 4, T_{c}=2 \mathrm{~s}$.

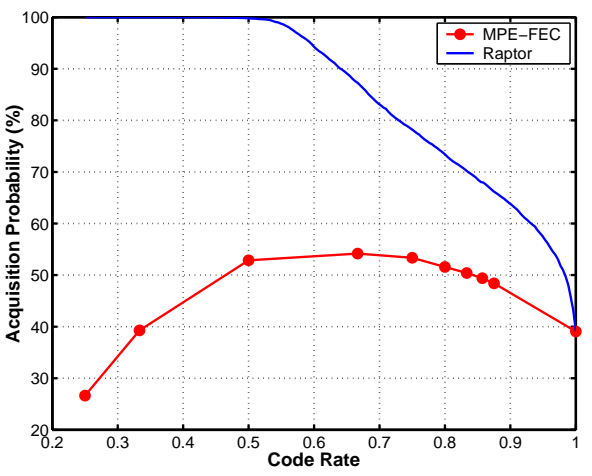

(b) $F_{S}=6 \mathrm{Mb}, B_{s}=1 \mathrm{Mb}, T_{c}=2 \mathrm{~s}$.

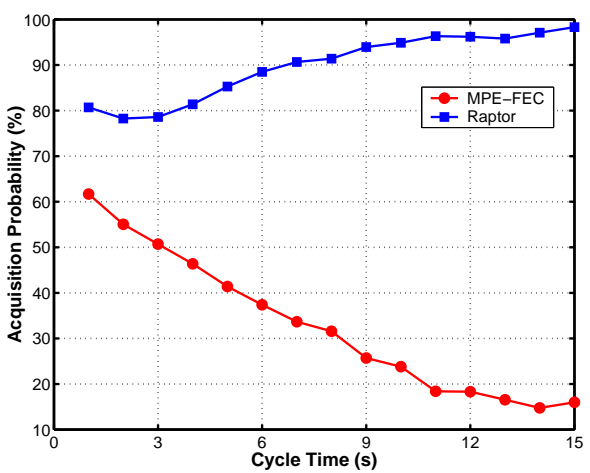

(d) $F_{S}=6 \mathrm{Mb}, C R=3 / 4, B_{s}=1 \mathrm{Mb}$.

Figure 3.8: File acquisition probability results for the vehicular traces show in Fig. 3.2 for different file sizes $\left(F_{S}\right)$, code rates $(C R)$, burst sizes $\left(B_{S}\right)$ and cycle time values between bursts $\left(T_{c}\right)$.

Figure 3.8(c) evaluates the influence of the burst size for code rate 3/4. It shows the acquisition probability of a $6 \mathrm{Mb}$ file as a function of the burst size. As expected, it can be seen that for MPE-FEC the acquisition probability is maximized for the maximum burst size of $2 \mathrm{Mb}$. The same reason than before applies: the number of bursts into which the file is partitioned is minimized and hence the performance maximized. With multi-burst FEC the acquisition probability is greatest for the smallest burst size. The reason is that the diversity gain increases despite the time interleaving is kept constant.

As multi-burst FEC provides protection over the whole file, for a constant download data rate the time interleaving equals to the transmission duration of the file and is independent on the burst size. But the diversity gain depends on the number of bursts jointly encoded within that time, and it increases for smaller burst sizes. The drawback is an increase in the battery power consumption of the terminals, as it is directly proportional to the number of bursts received. The optimum trade-off is thus the smallest burst size 
possible without compromising the power saving due to time-slicing and the capability of performing seamless handovers during the off-time. In the figure we can note that the improvement reducing the burst size is not very significant.

Finally, we investigate the effect of the cycle time between bursts for a burst size of $1 \mathrm{Mb}$. Figure 3.8(d) shows the file acquisition probability as a function of the cycle time. We can see that the acquisition probability increases with multi-burst FEC and reduces with MPE-FEC. The larger the time between bursts, the larger the crossed distance and the lower the correlation between reception conditions of consecutive bursts, increasing thus the diversity experienced by the users. Results obtained show that multi-burst FEC exploits this diversity, whereas MPE-FEC doesn't. In this sense, with MPE-FEC it is easier to deliver files in pedestrian environments and more difficult for motorway environments. Multi-burst FEC is always more efficient for files spanning several bursts, but the gain is higher in high-diversity vehicular scenarios.

\subsection{Fading Margin Gain Simulation Results}

In this section we evaluate the reduction in the average CNR required to cope with fast fading and shadowing (fading margin gain) that can be achieved with multi-burst FEC with Raptor coding in DVB-H. Multi-burst FEC yields a gain due to fast fading due to the improved capability for recovering form partially received bursts that MPE-FEC cannot, and also a gain due to shadowing because it is possible to recover from completely erroneous bursts. The problem is that the same parity data is used to correct both types of errors, and hence it is difficult to quantify the gain due to each type of fading. Before continuing reading this chapter, readers are encouraged to read first Appendix B, which contains a study of the fading margin reduction due to shadowing that can be achieved with multi-slot FEC in a generic time division multiplexing broadcast system.

Results have been obtained with the dynamic simulator described in Appendix A considering only fast fading and shadowing (i.e., without path loss). In the previous section we have seen that the gain of multi-burst FEC compared to MPE-FEC for a given latency and code rate depends on the distribution of the transmission errors over time. In this case the gain will depend on the degree of spatial diversity experienced by the user along the bursts jointly encoded. The lower the statistical correlation between reception conditions of the bursts corresponding to the same source block, the higher the gain. The maximum gain will thus occur when there is no correlation between reception conditions of consecutive bursts. Fast fading between different bursts can be considered uncorrelated, but shadowing is a spatial correlated process [120].

The simulation scenario is similar to the one employed in the standardization work of AL-FEC for filecasting services in DVB-H [57]. Basically, it assumes a user moving at a constant velocity across a lognormal CNR map defined by its average value and its standard deviation $\sigma_{l}$ and correlation distance $d_{\text {corr }}$ (resembling shadowing characteristics). The spatial correlation follows a first-order exponential model [121]. 
Table 3.2: Fading margin gain (dB) and area coverage extension (\%). $L_{d}=L_{0}+10 \cdot 3.5 \cdot \log _{10}[d]$.

\begin{tabular}{|c|c|c|c|c|c|c|c|c|c|c|}
\hline $1 \mathrm{~dB}$ & $2 \mathrm{~dB}$ & $3 \mathrm{~dB}$ & $4 \mathrm{~dB}$ & $5 \mathrm{~dB}$ & $6 \mathrm{~dB}$ & $7 \mathrm{~dB}$ & $8 \mathrm{~dB}$ & $9 \mathrm{~dB}$ & $10 \mathrm{~dB}$ & $11 \mathrm{~dB}$ \\
\hline \hline $14 \%$ & $30 \%$ & $48 \%$ & $69 \%$ & $93 \%$ & $120 \%$ & $151 \%$ & $187 \%$ & $227 \%$ & $273 \%$ & $325 \%$ \\
\hline
\end{tabular}

The correlation factor between consecutive bursts depends on the shadowing correlation distance, $d_{c o r r}$, the cycle time between bursts, $T_{c}$, and the user velocity, $v$ :

$$
F_{c o r r}=e^{-v \cdot T_{c} / d_{c o r r}}
$$

Fast fading is included in the DVB-H physical layer performance models. We employ models for the typical urban 6-tap (TU6) channel, which is representative for DVB-H vehicular reception for Doppler frequencies above $10 \mathrm{~Hz}$ (e.g., $18 \mathrm{~km} / \mathrm{h}$ at $600 \mathrm{MHz}$ ) [14], and for the pedestrian indoor (PI) channel model specifically developed for slowly moving DVB-H pedestrian reception [122]. Models were developed from laboratory measurements using the same DVB-H receivers employed in the field measurements in Turku following the procedure described in Appendix A.3. The physical layer transmission mode considered is: FFT size $8 \mathrm{~K}$, OFDM symbol guard interval (GI) 1/4, modulation 16-QAM and code rate 1/2; which provides a channel data rate of $10 \mathrm{Mb} / \mathrm{s}$. It should be pointed out that very similar results are achieved for different transmission modes (e.g., modulation order QPSK, code rate 2/3).

\section{Coverage Gain}

Fading margin gains can be translated into coverage expansion gains because they can be directly included in the link budget. Assuming that the received signal strength decreases exponentially with the distance, with distance dependent path loss $L_{d}$ :

$$
L_{d}=L_{0}+10 \cdot \alpha \cdot \log _{10}[d]
$$

where $L_{0}$ is the distance independent term which represents the path loss at $1 \mathrm{~km}$ distance, $\alpha$ is propagation exponent, and $d$ is the distance in $\mathrm{km}$. The relative increase in the maximum distance supported (cell radius) for a given link margin gain in $\mathrm{dB}, \Delta L$, can be computed as:

$$
\frac{R+\Delta R}{R}=10^{\Delta L /(10 \cdot \alpha)} .
$$

Assuming a circular or hexagonal shape of the cell, the gain in area coverage is equal to the square of Equation (3.3). Table 3.2 shows the coverage extension as a function of the link margin gain for $\alpha=3.5$ (typical urban environment). Note that a $3 \mathrm{~dB}$ link margin gain means already a significant increase of the service area by $50 \%$, whereas a $5 \mathrm{~dB}$ gain nearly doubles it. Shown results can be also interpreted in terms of the network infrastructure reduction that can be achieved to provide the same service area. For example, with a 5 $\mathrm{dB}$ link margin gain it would be possible to deploy a network with half the infrastructure investment and reach the same coverage area. 


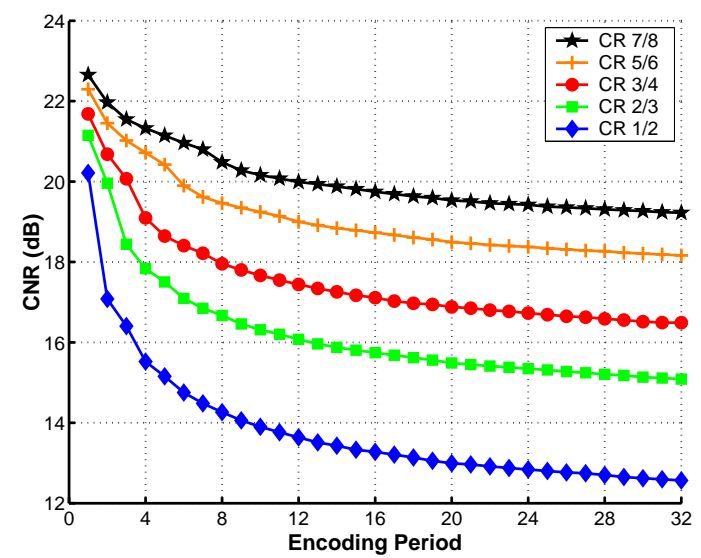

Figure 3.9: Average CNR for 5\% burst error rate vs. Encoding period. FFT 8K GI 1/4 16-QAM 1/2. TU6 channel $f_{d}=10 \mathrm{~Hz}$. Uncorrelated shadowing $\sigma_{l}=5.5 \mathrm{~dB}, d_{\text {corr }}=0 \mathrm{~m}$.

\subsubsection{Streaming Services}

For streaming we investigate the fading margin gain as a function of the code rate and the number of bursts jointly encoded (encoding period) to achieve a target burst error rate.

\section{Uncorrelated Shadowing}

Figure 3.9 shows the required average CNR to achieve a $5 \%$ burst error rate for different code rates as a function of the number of bursts jointly encoded with uncorrelated shadowing for $10 \mathrm{~Hz}$ Doppler. The burst size is adjusted such that a cycle time of $1 \mathrm{~s}$ is achieved for a $384 \mathrm{~kb} / \mathrm{s}$ streaming service. We can see that the potential gain is very significant, and that it increases for longer interleaving and for more robust code rates as expected. Note also that very interesting gains are feasible for relatively low encoding periods, especially for robust code rates such as $1 / 2$ and $2 / 3$.

It is worth to note that gains are so high that it can be more efficient to increase the modulation order at the physical layer and increase the protection at the upper layers to provide a target capacity. For example, in the figure it can be observed that for a code rate $1 / 2$ the CNR reduction is larger than $6 \mathrm{~dB}$ for encoding periods larger than 8 . For this code rate the channel data rate is reduced by half. The same capacity can be achieved reducing the modulation order from 16-QAM to QPSK, which CNR requirement is about $6 \mathrm{~dB}$ lower [34]. It should be taken into account, however, that reducing the modulation order affects all reception cases, including static reception, whereas the multi-burst FEC gain only applies for mobile users.

In addition to the code rate and encoding period, the gain depends on several factors. In particular, the gain increases for more demanding quality criteria. In our example gains for $1 \%$ burst error rate are between $1 \mathrm{~dB}$ and $3 \mathrm{~dB}$ larger. The gain due to shadowing is directly proportional to the shadowing standard deviation. The reason is that it determines 


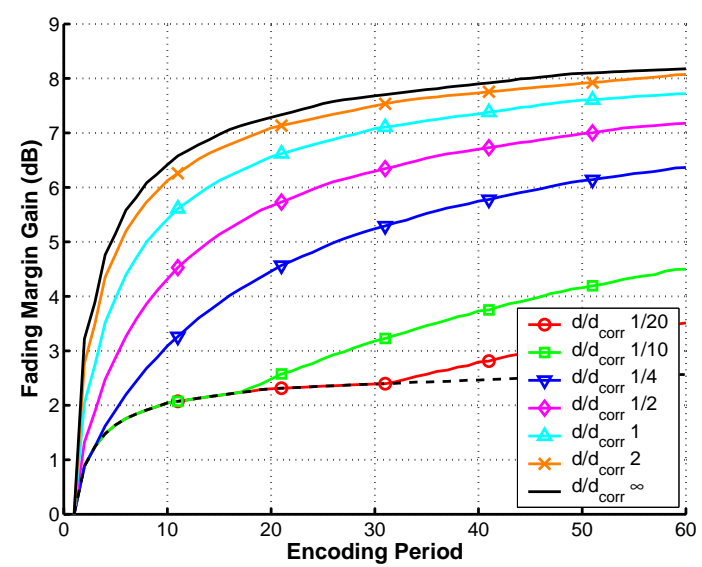

Figure 3.10: Fading margin gain for $5 \%$ burst error rate with code rate $1 / 2$ vs. Encoding period. FFT 8K, GI 1/4, 16-QAM 1/2. TU6 channel $f_{d}=10 \mathrm{~Hz}$. Correlated shadowing $\sigma_{l}=5.5 \mathrm{~dB}$.

the spread of the CNR values around the mean value being a direct measure of the spatial diversity perceived by the user $^{3}$. The gain due to fast fading is also higher for larger Doppler because of the degradation of the performance of the physical layer.

\section{Correlated Shadowing}

As for DVB-H streaming services the cycle time is usually in the range of one to few seconds, only vehicular users with very high speeds may experience uncorrelated shadowing between consecutive bursts. In practice gains will be smaller than the ones shown above.

Figure 3.10 shows the fading margin gain for a code rate $1 / 2$ as a function of the encoding period for different ratios between the moved distance by the user during the cycle time and the shadowing correlation distance, $d / d_{\text {corr }}$. In the figure it can be seen how the gain decreases when the correlation between consecutive bursts increases. However, there is a minimum gain due to fast fading (dash curve in the figure). If the correlation between the bursts jointly encoded is very high, there is no gain due to shadowing. It can be observed that the gain due to fast fading reaches a saturation level for an encoding period of 10 bursts. The gain due to shadowing increases the smaller the shadowing correlation distance and the larger the cycle time between bursts. Moreover, for a given scenario and transmission configuration, the gain also depends on the velocity of the users. It should be noted that lower velocities, larger correlation distances, and shorter cycle time values can be compensated employing a longer time interleaving. Therefore, these parameters basically determine the level of latencies required to achieve a given gain. It should be clear, however, that there is a maximum gain that is practically achieved when the receiver moves about one correlation distance between bursts.

\footnotetext{
${ }^{3}$ Gain values for a standard deviation of $8 \mathrm{~dB}$, which may be typical for dense urban scenarios are thus $45 \%$ larger than the ones presented in this chapter. For more details on this topic see Appendix B.
} 
Table 3.3: Multi-burst FEC gain (dB) due to fast fading for $10 \mathrm{~s}$ latency compared to MPE-FEC 3/4.

\begin{tabular}{|c|c||c|c|c|c|c|c|c|}
\hline \multirow{2}{*}{$\begin{array}{c}\text { Reception } \\
\text { Case }\end{array}$} & \multicolumn{1}{|c|}{$\begin{array}{c}\text { Error } \\
\text { Rate }\end{array}$} & $1 / 4$ & $1 / 3$ & $1 / 2$ & $2 / 3$ & $3 / 4$ & $5 / 6$ & $7 / 8$ \\
\cline { 3 - 9 } & & \multicolumn{7}{|c|}{ Code Rate } \\
\hline \hline Pedestrian & $1 \%$ & 3.0 & 2.7 & 2.3 & 1.8 & 1.5 & 1.1 & 0.8 \\
\cline { 2 - 9 } $3 \mathrm{~km} / \mathrm{h}$ & $5 \%$ & 2.5 & 2.3 & 1.9 & 1.4 & 1.1 & 0.7 & 0.5 \\
\hline $\begin{array}{c}\text { Vehicular } \\
18 \mathrm{~km} / \mathrm{h}\end{array}$ & $1 \%$ & 6.8 & 6.2 & 5.2 & 4.0 & 3.3 & 2.4 & 1.7 \\
\cline { 2 - 9 } & $5 \%$ & 5.3 & 4.8 & 3.8 & 2.6 & 2.0 & 1.1 & 0.5 \\
\hline
\end{tabular}

\section{Mobile TV Services}

For conventional mobile TV services the multi-burst FEC gain is limited in order to provide short zapping times. No more than 10-15 seconds latency are expected to be concealed from the perception of the users implementing so-called fast zapping techniques, see Chapter 2.3.5. In this case, the multi-burst FEC gain basically stems mainly due to fast fading. Only for vehicular users at high speeds in scenarios with low/moderate correlation distances will be able to benefit from temporary shadowing.

Table 3.3 shows the multi-burst FEC gain due to fast fading compared to MPE-FEC 3/4 for 10 seconds latency as a function of the code rate for pedestrian and vehicular reception cases. Constant velocities of $3 \mathrm{~km} / \mathrm{h}$ and $18 \mathrm{~km} / \mathrm{h}$ have been assumed, respectively $(1.6 \mathrm{~Hz}$ and $10 \mathrm{~Hz}$ Doppler at $600 \mathrm{MHz}$ ). Two target burst error rates are considered, $5 \%$ and $1 \%$. For $1 \%$ burst error rate the gain increases about $0.5 \mathrm{~dB}$ for pedestrian users and about 1.5 $\mathrm{dB}$ for vehicular users.

In the table we can see that very important gains are feasible for vehicular reception. In this case the area coverage can be easily doubled. Moreover, depending on the shadowing correlation distance and user velocity it may be possible to achieve even larger gains due to shadowing. For example, $18 \mathrm{~km} / \mathrm{h}$ there is no shadowing gain for $100 \mathrm{~m}$ correlation distance (typical value for sub-urban environments). For only $20 \mathrm{~m}$ distance, which may be representative for dense urban areas, up to 2 additional $\mathrm{dB}$ gain can be achieved for $1 / 4$ code rate. For pedestrian reception interesting gains due to fast fading are only feasible for very robust code rates, especially if very high service quality is targeted. In this case the area coverage can be increased up to $50 \%$. For pedestrian reception there is no gain due to shadowing because of the reduced mobility of the users.

Finally, it should be noted that introducing multi-burst FEC instead of MPE-FEC in a given network keeping the same capacity (number of channels) with a code rate $3 / 4$ would increase the area coverage between $50 \%$ for vehicular users and $20 \%$ for pedestrian users. 


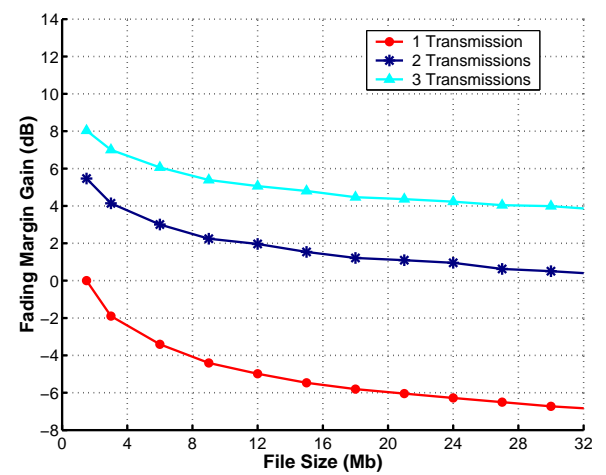

(a) MPE-FEC 3/4.

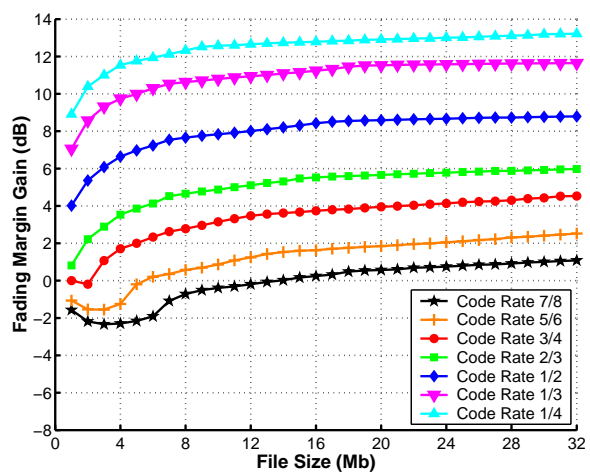

(b) Raptor.

Figure 3.11: Fading margin gain for $95 \%$ file acquisition probability vs. File size. FFT 8K GI 1/4 16-QAM 1/2. TU6 $f_{d}=10 \mathrm{~Hz}$. Uncorrelated shadowing $\sigma_{l}=5.5 \mathrm{~dB}, d_{\text {corr }}=0 \mathrm{~m}$.

\subsubsection{File Download Services}

For file download services the fading margin gain it is defined as the difference between the reference CNR threshold for conventional streaming services (i.e., CNR requirement for 5\% burst error rate with MPE-FEC code rate and burst size $2 \mathrm{Mb}$ ) and the required CNR to achieve a $95 \%$ file acquisition probability [57].

For MPE-FEC we evaluate the fading margin as a function of the file size. We consider a code rate of $3 / 4$ and a burst size of $2 \mathrm{Mb}$ in order to minimize the number of bursts where the files are partitioned. For Raptor codes we evaluate the fading margin as a function of the file size and code rate. In this case there is no optimum code rate, but assuming a fixed download data rate the performance improves for smaller burst sizes as the diversity gain is increased. Here we consider a constant burst size of $1 \mathrm{Mb}$. Smaller burst sizes do not provide significant improvements and they increase the battery power consumption.

\subsubsection{Uncorrelated Shadowing}

Figure 3.11 shows the fading margin gain as a function of the file size for MPE-FEC when the file is transmitted once, twice and three times, and for different code rates with multiburst FEC. In the figure we can notice that MPE-FEC suffers a penalization for large files. The fading margin gain is negative for the case with one single file transmission for files spanning more than one burst. This means that the area coverage is reduced compared to conventional streaming services. In contrast, with multi-burst FEC the area coverage for file download services is higher than streaming services with MPE-FEC. The reason is that it is possible to benefit from the time diversity of the mobile channel during the transmission of the files that span several bursts. The gain for large files is thus twofold, because MPE-FEC performs worse and multi-burst FEC performs better. The minimum multi-burst FEC gain occurs for files that consist of a single burst. 


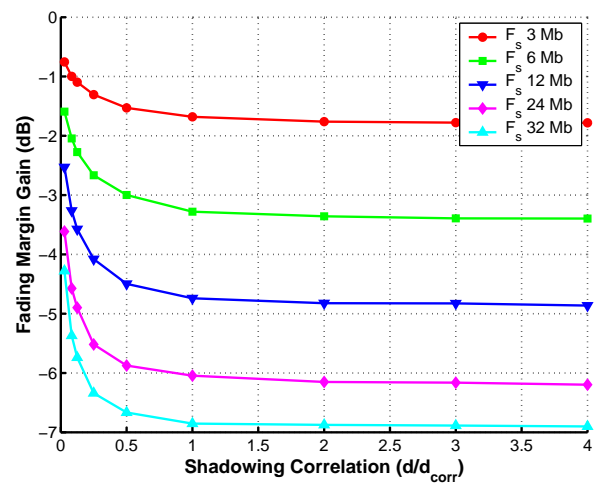

(a) MPE-FEC 3/4.

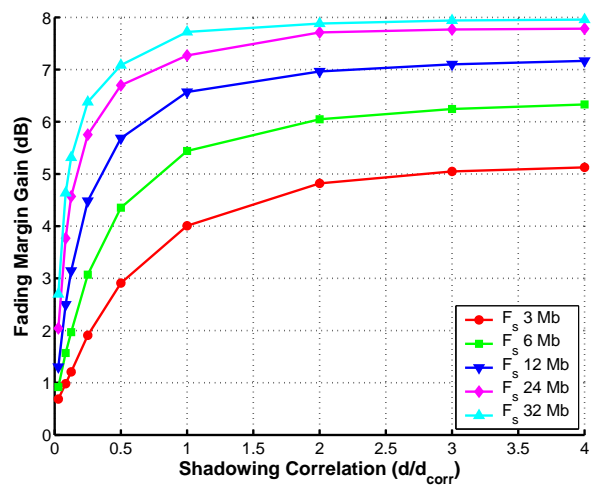

(b) Raptor $1 / 2$.

Figure 3.12: Fading margin gain for $95 \%$ file acquisition probability vs. Shadowing correlation between consecutive bursts $\sigma_{l}=5.5 \mathrm{~dB}$. FFT 8K GI 1/4 16-QAM 1/2. TU6 $f_{d}=10 \mathrm{~Hz}$.

With multi-burst FEC the coverage improvement is also higher for more robust code rates. Similarly than for streaming services, the gain increases for larger standard deviations of the shadowing and for more demanding acquisition probabilities. In the figure it can be observed that for very high code rates there is a penalization for small files that span few bursts. The reason is that they are more vulnerable to lost bursts (e.g., with a coding rate $7 / 8$ it is not possible to recover from one completely erroneous burst for encoding periods smaller than 8).

One possibility to increase the coverage with MPE-FEC is retransmitting the file in a carousel. The effective code rate with one and two file retransmissions are 3/8 and 1/4 respectively. Compared to multi-burst FEC, the problem is that it is possible that terminals receive duplicate packets which are useless. Hence, the efficiency of the additional data transmitted is considerably lower.

\section{Correlated Shadowing}

As for streaming services the maximum multi-burst FEC gain for file download services occurs when there is no correlation between reception conditions of two consecutive timesliced bursts. If there is correlation the gain decreases as the time diversity of the mobile channel is reduced. The gain will be thus smaller for lower user velocities, larger values of the shadowing correlation distances, and shorter cycle times between bursts. However, contrarily to streaming services, file download services do not usually have strict latency constraints, and thus a relatively larger cycle time value can be employed.

Figure 3.12 shows the evolution of the fading margin gain as a function of the shadowing correlation between consecutive bursts. It is worth to note that a larger correlation is beneficial for MPE-FEC, and the required fading margin decreases when the correlation increases. However, there is a minimum degradation due to fast fading. 


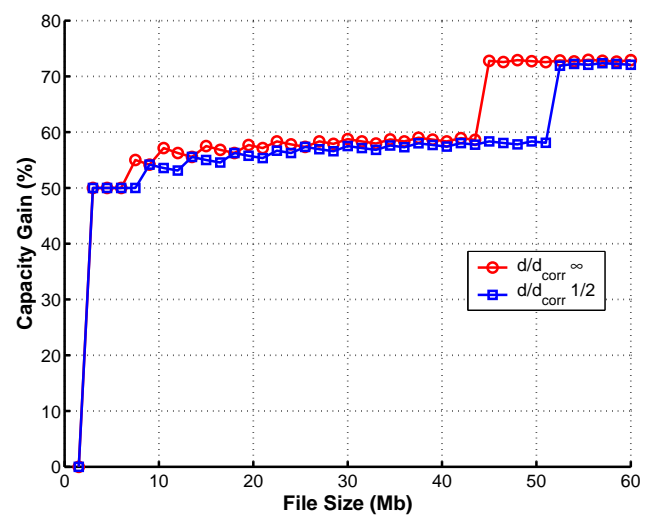

Figure 3.13: Capacity gain for $95 \%$ acquisition probability vs. File size. FFT 8K GI 1/4 16-QAM 1/2. TU6 $f_{d}=10 \mathrm{~Hz}$. Correlated shadowing $\sigma_{l}=5.5 \mathrm{~dB}$.

\section{Capacity Gain}

For file download services the gain of multi-burst FEC compared to MPE-FEC can be also expressed as a capacity gain due to a reduction of the delivery time (number of bursts) required to achieve a target acquisition probability. We define the capacity gain as the proportion of number of bursts saved with multi-burst FEC compared to MPE-FEC 3/4 to achieve a $95 \%$ file acquisition probability. The CNR is fixed, such that the burst error rate with MPE-FEC $3 / 4$ is 5\%. With MPE-FEC there is no other option than transmitting the whole file several times until the desired acquisition probability is achieved. In contrast, with multi-burst FEC the number of bursts required is directly proportional to the file size because it is possible to transmit additional bursts with parity data (the standardized version of Raptor codes adopted in DVB-H supports at least a code rate equal to 1/8).

Figure 3.13 shows the capacity gain as function of the file size for the case with uncorrelated shadowing and one case with correlation. We can see that there are two gain areas. In the first area the gain takes values between $50 \%$ and $60 \%$. In this range one file retransmission is needed with MPE-FEC. In the second area two file retransmissions with MPE-FEC are required, and the gain takes values about $70 \%$. The file size where the transition between two and three retransmissions occurs depends on the shadowing correlation. This threshold increases the higher the correlation is. Results have been verified with the analytical framework for uncorrelated shadowing derived in Appendix B. The file size, in number of bursts, that requires a second retransmission to achieve a $95 \%$ acquisition probability for a $5 \%$ burst error rate is 21 , which would correspond to $31.5 \mathrm{Mb}$ in our example. The reason why the transition between two and three retransmissions with MPE-FEC takes place for larger files in our example is that we take into account partially received bursts. 


\subsection{Conclusions}

In this chapter we have evaluated the performance of multi-burst forward error correction with Raptor coding for DVB-H streaming and file download services compared to the conventional MPE-FEC mechanism. Multi-burst FEC exploits the time diversity of the mobile channel at the expense of increasing the network latency to enhance the FEC coding efficiency to recover from temporary transmission errors (being even possible to recover from lost burst). The transmission robustness can be improved trading both system capacity and network latency. The gain increases for more robust code rates and larger number of bursts jointly encoded, but it depends on the time evolution of the transmission errors experienced by the users, and hence it depends on the velocity and trajectory of the users.

Field measurement results reveal that the potential gain is very high, for both vehicular and pedestrian reception cases, although robust code rates such as $2 / 3$ or $1 / 2$ are necessary. MPE-FEC is only effective in very dense SFN networks with very good coverage level over the service area to compensate for the fast fading due to the mobility of the users. In contrast, multi-burst FEC can also cope with temporary shadowing and outage because it can recover from completely erroneous bursts. Multi-burst FEC is especially suited for vehicular users because of their high speeds, achieving very significant gains for relatively small latencies (around 10-15 seconds) that may be acceptable for conventional mobile TV services implementing fast zapping techniques. Pedestrian users, on the other hand, require large latencies due to their reduced mobility, especially to cope with temporary lack of coverage.

Our simulation results show that very relevant reductions in the fading margin are feasible. Multi-burst FEC provides an improved protection against fast fading because it can recover from heavily corrupted bursts that MPE-FEC cannot, but it can also partially cope with temporary shadowing due to its capability of recovering from lost bursts. This fading margin gain heavily depends on the shadowing correlation between the reception conditions of the bursts jointly encoded, which depends on the transmission configuration (number of bursts jointly encoded and cycle time between bursts), the user (velocity), and the shadowing characteristics (standard deviation ${ }^{4}$ and correlation distance). If the correlation is very high (e.g., for few bursts jointly encoded, for very low user velocities, or for very short cycle time values), there is no gain due to shadowing and the gain only stems due to fast fading. On the other hand, if the correlation is low, the gain can be significantly increased due to shadowing. The potential gain is so high that it can be more efficient to increase the modulation order at the physical layer and increase the protection at the upper layers to provide a target capacity. However, the multi-burst FEC gain only applies for mobile users on the move.

${ }^{4}$ The gain is directly proportional to the shadowing standard deviation, see Appendix B. The gain also depends on the target error rate, and it increases for more demanding criteria. 
File download services exhibit the largest gains because it is possible to encode large files into a single source block (e.g., $64 \mathrm{Mb}$ ) and to reduce the shadowing correlation level increasing the cycle time between bursts. But also because with MPE-FEC it is more difficult to deliver large files that span several bursts. With MPE-FEC the area coverage for file download services is smaller than for streaming services. As a consequence, an efficient delivery of large files is only possible in the areas where the burst error rate (with MPE-FEC) is close to zero. Compared to streaming services with MPE-FEC, multi-burst FEC can easily yield fading margin gains over $10 \mathrm{~dB}$ for file download services which would treble the area coverage. The multi-burst FEC gain can be also expressed as a capacity gain due to a reduction of the delivery time (number of bursts) required to achieve the same coverage for file download services with MPE-FEC. The capacity gain takes values between $50 \%$ and $75 \%$ if the same coverage than for streaming services with MPE-FEC is targeted.

For conventional mobile TV streaming the multi-burst FEC gain is limited because cycle time values are very short (in the order of one or two seconds), and the number of bursts that can be jointly encoded is reduced. In this case the gain stems mainly due to fast fading. The gain is not very high for pedestrian users, but it is important for vehicular users. For example, introducing multi-burst FEC with a typical MPE-FEC code rate 3/4 in an existing network would increase the area coverage between $50 \%$ for vehicular users and $20 \%$ for pedestrian users while keeping the same capacity (number of TV services). For more robust code rates the area coverage can be at least doubled for vehicular reception, whereas for pedestrian reception it can be increased up to $50 \%$. Vehicular users with very high speed may experience larger gains due to shadowing. 


\section{Chapter 4}

\section{DVB-H Deployment on Existing Wireless Infrastructure}

In the previous chapter, we have seen that multi-burst FEC can improve the DVB-H transmission robustness significantly. The gain can be expressed as a reduction in the fading margin to cope with fast fading and shadowing. However, these evaluations assume a constant velocity across the bursts jointly encoded, which is not realistic, especially for moderate and large latencies. In practice, users may become temporarily static due to traffic lights for example. For static users the multi-burst FEC gain stems from the dynamic variations in the surroundings of the receiver environment. This gain is considerably smaller than the gain obtained due to the mobility of the users, and it can be even negligible if the user stops in a spot with a reduced coverage. For large latencies and high user velocities path loss variations may also play an important role. This is particularly cumbersome from a network planning point of view, because it implies that the gain depends on the trajectory and velocity of the users. In this chapter we present a methodology to estimate the coverage for multi-burst FEC services in DVB-H networks based on dynamic system-level simulations taking into account realistic mobility patterns. We also investigate the coverage gain in a realistic urban scenario.

The chapter is structured as follows. First, we discuss the feasibility of DVB-H deployment on existing wireless infrastructure for digital broadcasting and cellular systems in Section 4.1. Next, we investigate the scalability of DVB-H deployment in capacity and coverage in Section 4.2. In Section 4.3 we highlight the main characteristics of repeaters (gap-fillers), and summarize the considerations that should be taken into account during the network planning phase. Section 4.4 presents and describes the use of a multi-objective genetic algorithm to find cost minimized and coverage maximized DVB-H network configurations. Section 4.5 is devoted to coverage estimation of multi-burst FEC services in realistic scenarios. The chapter is concluded with Section 4.6. 
Table 4.1: Main link budget differences between DVB-T and DVB-H: receiver antenna gain $G_{r x}$, feeder loss $L_{f}$, building penetration loss $L_{b}$, vehicle penetration loss $L_{v}$, height loss at $1.5 \mathrm{~m} L_{h}$, and fading margin $C_{l}$.

\begin{tabular}{|c|c|c|c|c|c|c|}
\hline & \multirow{2}{*}{$\begin{array}{l}\text { DVB-T } \\
\text { Rooftop }\end{array}$} & \multicolumn{3}{|c|}{ DVB-H Pedestrian } & \multicolumn{2}{|c|}{ DVB-H Vehicular } \\
\hline & & Outdoor & Light Indoor & Deep Indoor & In-Car & Rooftop \\
\hline$G_{r x}$ & $13.5 \mathrm{dBi}$ & \multicolumn{4}{|c|}{$-7 \mathrm{dBi}$ (built-in antenna), $-3 \mathrm{dBi}$ (attached antenna) } & $0 \mathrm{dBi}$ \\
\hline$L_{f}$ & $5 \mathrm{~dB}$ & - & - & - & - & - \\
\hline$L_{b}$ & - & - & $11 \mathrm{~dB}$ & $17 \mathrm{~dB}$ & - & - \\
\hline$L_{v}$ & - & - & - & - & $7 \mathrm{~dB}$ & - \\
\hline$L_{h}$ & - & \multicolumn{5}{|c|}{12 (rural), 18 (suburban), $24 \mathrm{~dB}$ (urban) } \\
\hline$C_{l}$ & $9 \mathrm{~dB}$ & $9 \mathrm{~dB}$ & $12 \mathrm{~dB}$ & $13 \mathrm{~dB}$ & $13 \mathrm{~dB}$ & $13 \mathrm{~dB}$ \\
\hline
\end{tabular}

\subsection{Feasibility of DVB-H Infrastructure Deployment}

\section{Link Budget}

We can see that the overall link budget difference is well over $30 \mathrm{~dB}$ in urban scenarios. The difference is partly compensated because DVB-H networks employ more robust transmission modes with lower capacities, but still the differences are very significant. For instance, the most common DVB-T transmission mode in Europe with system parameters: FFT size $8 \mathrm{~K}$, OFDM symbol guard interval 1/8, modulation 64-QAM, and code rate 2/3; provides a capacity of $22.2 \mathrm{Mb} / \mathrm{s}$ (usually six TV channels per multiplex) and its CNR requirement for rooftop reception is $20.1 \mathrm{~dB}$. On the other hand, typical CNR requirements for DVB-H range between $7.5 \mathrm{~dB}$ (QPSK 1/2 MPE-FEC 3/4) and $16.6 \mathrm{~dB}$ (16-QAM 2/3 MPE-FEC 3/4), with capacities between 4 and $11 \mathrm{Mb} / \mathrm{s}$.

Existing broadcasting DVB-T towers dimensioned for fixed rooftop reception will therefore provide very limited DVB-H coverage ${ }^{1}$, and a considerably large number of new sites will be required for the installation of additional transmitters or gap-fillers. As investment in new infrastructure is very costly, reusing existing infrastructure for cellular networks may be key to minimize the deployment cost of DVB-H [36].

\section{Estimation of Cellular Sites Needed}

To provide an insight into the amount of sites that may be needed, we investigate the maximum cell radius for typical cellular sites. We assume an urban Okumura-Hata path loss propagation model [123].

\footnotetext{
${ }^{1}$ DVB-T networks planned for portable reception such as Germany take into account the penalization due to height loss and lower gain antennas [33]. Therefore, the difference is considerably reduced. In these cases the differences come from the antenna gain and the CNR requirement. DVB-H mobile reception with MPE-FEC requires a couple of $\mathrm{dB}$ more in $\mathrm{CNR}$ than portable DVB-T reception.
} 
Table 4.2: Typical DVB-H cell radius (km). FFT 8K GI 1/4 16-QAM 2/3 MPE-FEC 3/4.

\begin{tabular}{|c|c||c|c|c|c|c|}
\hline \multicolumn{2}{|c||}{ Site } & \multicolumn{3}{c|}{ Pedestrian } & \multicolumn{2}{c|}{ Vehicular } \\
\hline Power & Height & Outdoor & Light Indoor & Deep Indoor & In-Car & Rooftop \\
\hline \hline \multirow{2}{*}{$30 \mathrm{dBW}$} & $35 \mathrm{~m}$ & 1.5 & 0.6 & 0.3 & 0.7 & 1.7 \\
\cline { 2 - 7 } & $50 \mathrm{~m}$ & 1.7 & 0.7 & 0.4 & 0.8 & 2 \\
\hline \multirow{2}{*}{$33 \mathrm{dBW}$} & $35 \mathrm{~m}$ & 1.8 & 0.7 & 0.4 & 0.8 & 2 \\
\cline { 2 - 7 } & $50 \mathrm{~m}$ & 2.1 & 0.8 & 0.5 & 1.0 & 2.4 \\
\hline
\end{tabular}

The path loss, $L_{d}$, is computed as:

$$
\begin{aligned}
L_{d} & =L_{0}+10 \cdot \alpha \cdot \log _{10}[d] \\
L_{0} & =69.55+26.16 \cdot \log _{10}[f]-13.82 \cdot \log _{10}\left[h_{t x}\right]-a . \\
a & =h_{r x} \cdot\left(1.1 \cdot \log _{10}[f]-0.7\right)-\left(1.56 \cdot \log _{10}[f]-0.8\right) \\
\alpha & =\left(44.9-6.55 \cdot \log _{10}\left[h_{t x}\right]\right) / 10
\end{aligned}
$$

Where $d$ is the distance in $\mathrm{km}, f$ is the carrier frequency in $\mathrm{MHz}$, and $h_{t x}$ and $h_{r x}$ are the transmitting and receiving antenna height in meters respectively.

Table 4.2 shows the cell radius for the different reception cases considered above for two antenna site heights ( 35 and $50 \mathrm{~m}$ ) and two transmit powers ( $30 \mathrm{dBW}$ and $33 \mathrm{dBW})$. We consider the transmission mode: FFT 8K GI 1/4 16-QAM 2/3 at the physical layer, and MPE-FEC 3/4 at the link layer. It provides $10 \mathrm{Mb} / \mathrm{s}$ (envisaged commercial requirement [13]), and requires about 16-17.5 dB CNR depending on the reception case. DVB-H terminals are characterized with a built-in omni-directional antenna of $-7 \mathrm{dBi}$ gain, except for vehicular rooftop reception that $0 \mathrm{dBi}$ gain is assumed, and a noise figure of 6 $\mathrm{dB}$ (including a GSM reject filter). An additional $3 \mathrm{~dB}$ loss is considered to account for implementation losses and man-made noise [34].

From this simple example it is clear that very dense SFNs with a large number of sites are required, especially to achieve indoor and vehicular in-car coverage. In a dense SFN many receiving locations are served by more than one site, introducing redundancy in the transmitted signal and improving the coverage (also known as SFN gain), especially when pedestrian indoor reception and vehicular in-car reception are required. In the table we can also note the importance of re-using the highest sites possible. In this case increasing the antenna height from 35 to $50 \mathrm{~m}$ is equivalent to increase the transmit power $3 \mathrm{~dB}$.

From a business perspective, the bottleneck is quite clear: sufficiently large numbers of users are required for every site to eventually recover the investment in the infrastructure without charging unacceptable prices for the services [39]. High capacity and full coverage will be economically justified only in areas where the user density is very high. In areas with a scarce user population, the investment might never be recovered, meaning that only a low density of sites and a low to moderate capacity can be supported. 
Table 4.3: Trade-off capacity vs. robustness in DVB-H. MPE-FEC 3/4.

\begin{tabular}{|c|c|c|c|c|c|}
\hline \multirow{2}{*}{$\begin{array}{l}\text { Modulation } \\
\text { and code rate }\end{array}$} & \multirow{2}{*}{$\begin{array}{l}\text { CNR } \\
(\mathrm{dB}) \\
\end{array}$} & \multicolumn{4}{|c|}{ Capacity $(\mathrm{Mb} / \mathrm{s})$} \\
\hline & & GI $1 / 4$ & GI $1 / 8$ & GI $1 / 16$ & GI $1 / 32$ \\
\hline QPSK $1 / 2$ & 7.5 & 3.7 & 4.1 & 4.4 & 4.5 \\
\hline QPSK 2/3 & 11 & 5.0 & 5.5 & 5.9 & 6.0 \\
\hline 16-QAM 1/2 & 13.5 & 7.5 & 8.3 & 8.8 & 9.0 \\
\hline 16-QAM 2/3 & 16.6 & 10.0 & 11.1 & 11.7 & 12.0 \\
\hline 64-QAM 1/2 & 18.3 & 11.2 & 12.4 & 13.2 & 13.5 \\
\hline 64-QAM 2/3 & 21.4 & 15.0 & 16.6 & 17.6 & 18.0 \\
\hline
\end{tabular}

However, avoiding full DVB-H area coverage and very high capacity from the beginning is probably the key to providing affordable DVB-H services [38]. The combination of DVB-H with a cellular network into a hybrid cellular and broadcasting system allows to perform an incremental deployment of the DVB-H infrastructure over time, following the user demand and adding sites only where required and when needed. In the initial phases of the DVB-H network roll-out the cellular network can be used in low populated areas until the deployment of a DVB-H transmitter or gap-filler is economically justified. Moreover, it may not be necessary to initially provide such high broadcast capacity as the envisaged DVB-H commercial requirement, such that the area coverage can be enlarged. For example, reducing the system capacity from $10 \mathrm{Mb} / \mathrm{s}$ down to $5 \mathrm{Mb} / \mathrm{s}$ (decreasing the modulation order at the physical layer from 16-QAM to QPSK), would approximately double the area coverage per site. The coverage could be further increased with multi-burst FEC, increasing the protection at the upper layers as shown in the previous chapter.

\subsection{Scalability of DVB-H Infrastructure Deployment}

In this section we investigate what happens with the infrastructure cost per $\mathrm{Mb} / \mathrm{s}$ when the network migrates towards higher capacity and coverage. We are interested to know whether this cost decreases, remains constant or increases, when the service capacity and the coverage level are increased over a given service area. The economic success of a progressive DVB-H network deployment will be partly determined by the dynamics of the cost per $\mathrm{Mb} / \mathrm{s}$. The cost performance measure of interest is the cost per $\mathrm{Mb} / \mathrm{s}$ as a function of the infrastructure upgrade (transmission power and number of sites). An ideal situation would be to experience economies of scale. That is, the value of cost per Mb/s decreases when more capacity is provided over the service area.

Table 4.3 shows the capacity-robustness trade-off for selected transmission modes: modulations (QPSK, 16-QAM, 64-QAM) and code rates $(1 / 2,2 / 3)$ at the physical layer, and MPE-FEC 3/4 at the link layer. CNR values are taken from [124]. We will discuss the scalability properties of DVB-H based on this trade-off. Scalability results of a network planning exercise in a synthetic scenario can be found in [37]. 
Table 4.4: Coverage reduction (\%) due to CNR threshold increase (dB). $L_{d}=L_{0}+10 \cdot 3.5 \cdot \log _{10}[d]$.

\begin{tabular}{|c|c|c|c|c|c|c|c|c|c|c|}
\hline $1 \mathrm{~dB}$ & $2 \mathrm{~dB}$ & $3 \mathrm{~dB}$ & $4 \mathrm{~dB}$ & $5 \mathrm{~dB}$ & $6 \mathrm{~dB}$ & $7 \mathrm{~dB}$ & $8 \mathrm{~dB}$ & $9 \mathrm{~dB}$ & $10 \mathrm{~dB}$ & $11 \mathrm{~dB}$ \\
\hline \hline$-12 \%$ & $-23 \%$ & $-33 \%$ & $-41 \%$ & $-48 \%$ & $-55 \%$ & $-60 \%$ & $-65 \%$ & $-69 \%$ & $-73 \%$ & $-77 \%$ \\
\hline
\end{tabular}

\section{Scalability of System Capacity}

In Table 4.3 we can see that doubling the capacity, from QPSK 1/2 to 16-QAM 1/2 for example, implies $6 \mathrm{~dB}$ in $\mathrm{CNR}^{2}$. If the network infrastructure upgrade is performed only in transmission power all sites should increase their power by that amount. The problem is that for very large powers the annualized cost of power amplifiers, antenna system and expenses for electricity are dominant, such that the cost becomes proportional to the power. Therefore, the scalability is very bad (doubling the capacity would cost four times more), and the required Watts per $\mathrm{Mb} / \mathrm{s}$ increase when the modulation order increases. Trebling the capacity from QPSK 1/2 to 64-QAM 1/2 implies almost $11 \mathrm{~dB}$ in CNR. If the cost is proportional to the transmit power, it would be about 10 times more expensive! The reason for that behavior is the logarithmic dependence of the data rate with the CNR.

On the other hand, the system scalability when deploying new sites depends on the reduction of the area coverage per site when increasing the capacity. Differences in CNR can be translated into coverage expansion/reduction because they can be directly included in the link budget. In Section 3.3.1, we have shown that the gain in area coverage for a given link margin gain in $\mathrm{dB}, \Delta L$, can be approximated as $10^{2 \cdot \Delta L /(10 \cdot \alpha)}$, with $\alpha$ being the propagation exponent. Typical values of $\alpha$ are between 3 and 4 . For example the Okumura-Hata propagation model yields $\alpha=3.5$ for $35 \mathrm{~m}$ site height and $\alpha=3.4$ for 50 $\mathrm{m}$ height at $600 \mathrm{MHz}$.

Table 4.4 shows the coverage reduction as a function of the increase in the CNR threshold for $\alpha=3.5$. We can see that an increase of about 5.5 dB in the CNR threshold means a reduction of the area coverage per site by half. Therefore, the number of sites required with 16-QAM would be approximately double than with QPSK. The infrastructure cost of doubling the capacity would be double. For this case the scalability when moving towards 64-QAM is not good. For trebling the capacity it would be needed about four times the number of sites (11 dB in CNR).

A more scalable deployment in capacity is thus achieved by deploying new sites. Good scalability figures in capacity up to the $10 \mathrm{Mb} / \mathrm{s}$ commercial requirement can be achieved with a cost per Mb/s approximately constant. It should be pointed out that a more scalable deployment may be achieved by increasing the number of cellular sites and their transmit power, since the assumption that the cost is proportional to the power is only valid for large powers (i.e., broadcasting TV towers). Unfortunately, for this study it is necessary to quantify the cost weight of both alternatives, which requires confidential information from network operators.

${ }^{2}$ It can be observed that code rate $2 / 3$ provides worse performance than $1 / 2$. It provides a capacity $33 \%$ higher but it requires more than $3 \mathrm{~dB} C N R$. The reason is that the code rate $2 / 3$ is achieved by puncturing, being the mother code at the physical layer $1 / 2$. 


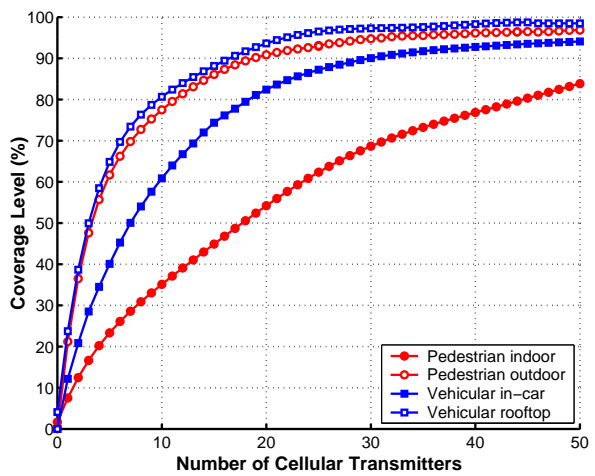

(a) System capacity $10 \mathrm{Mb} / \mathrm{s}$.

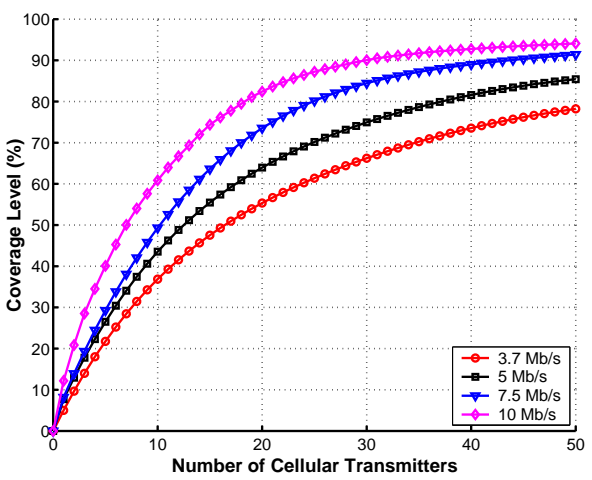

(b) Vehicular in-car reception.

Figure 4.1: Example of DVB-H network planning exercise in the city center of Valencia (Spain) using synchronized transmitters of $400 \mathrm{~W}$ at existing cellular sites.

\section{Scalability in Area Coverage}

Scalability in coverage is in general problematic for any wireless broadcast communications system that provides nearly full area coverage. The problem is that signal reception in very adverse conditions must be supported in order to maximize the potential number of subscribers that can receive the content. In the particular case of DVB-H, as its service availability is characterized by a very rapid transition from near perfect reception to no reception at all, it is especially costly to guarantee coverage to the last few per cent of the worst-served locations. This penalty is particularly evident for area coverage targets over $90 \%$ of service area locations. Network planning exercises in [40] over a wide area of 25 $\mathrm{km}$ radius using one broadcasting TV tower and cellular sites with synchronized transmitters show that providing $95 \%$ area coverage costs approximately $50 \%$ more than the cost of providing $90 \%$ area coverage. The cost can double if the target is set to $99 \%$ of locations.

Here, we show an example of a network planning exercise in the city center of Valencia (Spain), see Figure 4.1 [125]. The area of the deployment scenario is $15 \mathrm{~km}^{2}$, and 134 cellular sites have been considered as candidates to locate synchronized DVB-H transmitters of $400 \mathrm{~W}$. In Valencia the broadcasting TV towers are in the outskirts of the city, and the signal level at street level is very low because the DVB-T network is dimensioned for for fixed rooftop reception. Figure 4.1 shows the coverage level over the service area for different system capacities and reception cases as a function of the number of transmitters. We can see how it becomes very costly to cover the final percentages of locations. MPE-FEC 3/4 at the link layer has been assumed.

One solution to reduce the cost of covering the final percentages of locations is to use gap-fillers. Gap-fillers are on-channel repeaters that receive the transmitted RF signal from one site of the network and retransmit it at the same frequency after amplifying it. Compared to conventional synchronized transmitters, gap-fillers are lower cost solutions because they avoid the need of high cost equipment. 

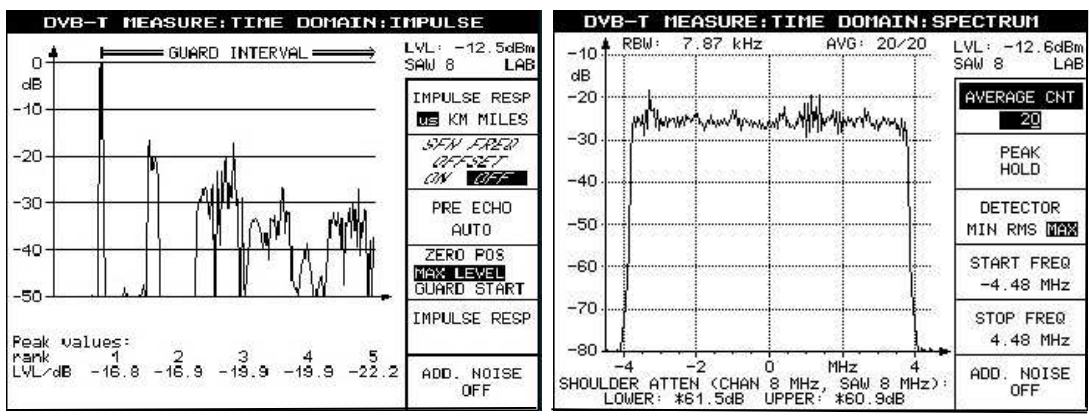

Figure 4.2: Impulse response and output signal frequency spectrum of a gap-filler without echo canceller. Margin gain $10 \mathrm{~dB}$.

Transmitters receive the base band signal by means of a dedicated transport network (terrestrial link, digital network or satellite link), modulate it, amplify it and transmit it. They are synchronized in both time and frequency using a GPS reference signal. Transmitters provide high quality in the transmitted signal and support high powers, but they may be a costly solution for a low-power site. Gap-fillers are lower cost solutions because they do not need a transport network, a COFDM modulator, and a GPS synchronization system. However, due to the coupling between the receiving and the transmitting antennas, the maximum output power is limited in order to avoid system oscillation (and hence the coverage level). Moreover, gap-fillers degrade the quality of the retransmitted signal as the signal is not regenerated, and an additional delay. Therefore, in general several gap-fillers will be needed to obtain the same area coverage than a transmitter, being key how cheaper the gap-fillers are compared to the transmitters to determine their suitability in the network deployment.

\subsection{Use of Gap-Fillers in DVB-H Networks}

Gap-fillers are feedback systems in which part of the transmitted signal is induced back to the input due to the coupling path between transmitting and receiving antennas [126], [127]. As a consequence, the transmitted signal consists on several echoes, each one delayed and attenuated from the previous one, with risk of instability and system oscillation, see Figure 4.2. It should be noted that the receiving antenna may receive unwanted echoes from different sites, and thus a very directive antenna should be used. The performance measure used to characterize the feedback is called margin gain. It is defined as the difference between the antennae isolation and the gap-filler gain, and corresponds to the relative attenuation between consecutive echoes.

The stability of the gap-filler is only guaranteed if the gain of the feedback-loop is lower than unity (i.e., the gain is lower than the isolation, or in terms of the gain margin, that is a positive value). This restriction limits significantly the gain, and hence the output power, of the gap-filler, and thus maximizing the isolation between antennas is essential to not reduce the area covered by the gap-filler. 

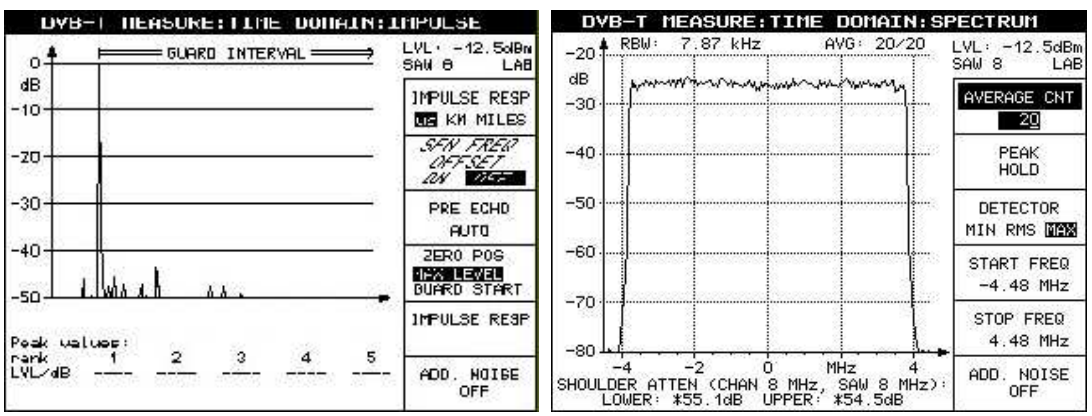

Figure 4.3: Impulse response and output signal frequency spectrum of a gap-filler without echo canceller. Margin gain $10 \mathrm{~dB}$.

The isolation depends mainly on the configuration of the antennas, and thus a careful design is crucial, but it also depends on the environment surrounding the site and the operating frequency. Some studies revealed that the isolation can vary from 15 up to 25 $\mathrm{dB}$ along the UHF band [128]. In general it is beneficial to increase the physical distance between antennas and protect the receiving antenna with architectural elements (e.g., panels). Another interesting possibility is to use different polarizations. On the other hand, the feedback-loop also degrades the quality of the transmitted signal introducing a ripple in the spectrum of the output signal (see Figure 4.2). The ripple depends on the gain margin, and for conventional gap-fillers a minimum value of $10 \mathrm{~dB}$ it is recommended [117].

Two quality indicator parameters are commonly used to characterize the degradation introduced by a gap-filler compared to a transmitter: MER (Modulation Error Ratio) and END (Equivalent Noise Degradation). The degradation is not only due to the gap-filler itself, but also because the received signal is degraded in the wireless radio link. The MER provides an overall quality indicator of the received and transmitted signals. Obviously, the MER of the transmitted signal cannot exceed the MER of the received signal as the signal is not regenerated. This limits the maximum number of gap-fillers that can be linked in a chain (usually no more than 2). The END provides a measure of the loss in the effective transmit power of the gap-filler, and it is used for network planning. It is defined as the increase in the CNR required to achieve a given quality error criteria compared to the use of a transmitter. Field and laboratory tests have shown that END values from 0.1 to $0.3 \mathrm{~dB}$ with respect to the input signals are feasible in static echoes conditions, increasing up to 2 $\mathrm{dB}$ with channel variations in semi-static environments [129].

The performance of gap-fillers can be considerably improved by means of echo cancellers, see Figure 4.3, being possible to safely operate the gap-filler with negative gain margin values of about $-10 \mathrm{~dB}$. That is, being possible to transmit 100 times more power [129]. Echo cancellers must be able to remove unwanted contributions to the received signal and to adapt to the changing channel, as it has been observed that in urban environments the isolation can present short-term fluctuations of up to $10 \mathrm{~dB}$. 
It should be also pointed out that the additional delay introduced by the gap-fillers is in the order of few $\mu \mathrm{s}(5-7 \mu \mathrm{s})$ [129], which is not relevant compared to the OFDM symbol guard interval employed in DVB-H SFN (up to $224 \mu \mathrm{s}$ ). From a network planning point of view, in order to use gap-fillers it is necessary to characterize each potential site not only with the isolation that can be achieved at, but also the potential degradation in terms of END and a feasible operating margin gain for the gap-filler.

\subsection{Network Planning Algorithm}

As a first step in the DVB-H network roll-out, existing broadcasting towers will be initially used to provide a basic coverage. They will be used at the maximum transmit powers possible to maximize the coverage level without interfering the existing analogue and digital TV transmissions. The network planning problem then consists in choosing, given a set of potential sites (mainly cellular sites, although the construction of new sites could be considered as well), the sites to be used with the type of emitter (transmitter or gap-filler) and its configuration ${ }^{3}$. Transmitters usually employ omnidirectional antennas (either with a single antenna or with a 3 sector configuration), and thus the only parameter is the transmit power. For gap-fillers, besides the transmit power, the azimuth (assuming a single sector transmitter antenna) and the emitter from which receive the signal are also important planning parameters.

To perform planning exercises it is required to estimate the coverage level in the target service area of each of the different possible network configurations, as well as a detailed cost model with the cost information of using each of the transmitters and gap-fillers considered in each potential site. In order to compute accurately the area coverage in a DVB-H SFN it is not only necessary an accurate prediction of the received power at each location from each transmission site in the network, but also to determine how signals from the different sites contribute to the useful received signal or cause self-interference at each location, see Annex A.2. The coverage performance measure in an SFN is the carrier-tointerference plus noise ratio (CINR) [130]. A particular receiving location is assumed to be covered if the CINR fulfils the so-called CNR requirement, which depends on the DVB-H transmission mode. It should be pointed out that the service availability with MPE-FEC is practically independent of the user speed [14]. The cost model should take into account transmit power, equipment (antennas, cables, etc.), electricity, site rental, maintenance, etc. The model must take into account both capital expenditures (CAPEX) and operational (OPEX) expenditures, and thus cost figures must be defined for a time period, as for example annualized costs (cost per year). Basically, depending on the type of emitter used, each site will have associated a cost for using it, and a cost proportional to the transmitted power, (transmitter or gap-filler). The possibility of building new sites could be considered by just including an additional cost factor.

\footnotetext{
${ }^{3}$ For a network planning exercise the target service area, the operating frequency and the transmission mode are given.
} 


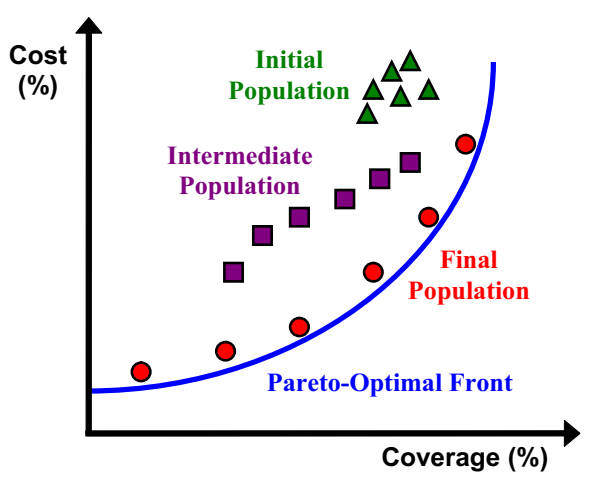

Figure 4.4: Evolution of the population of the genetic algorithm to the Pareto-optimal front.

Multi-objective genetic algorithms try to find, ideally, a set of solutions uniformly distributed along the Pareto-optimal front, as shown in Figure 4.4. Several variants can be found in the literature, being good examples the NSGA-II (Non-dominated Sorting Genetic Algorithm - version II) [131] or the SPEA2 (Strength Pareto Evolutionary Algorithm version 2) [132]. We adopt the NSGA-II algorithm because it performs slightly better (in the sense of achieving a broader spread of solutions) than SPEA2 when only two objectives are considered. Nevertheless, the approach adopted is generic, and allows applying any state-of-the-art multi-objective genetic algorithm [64]. The NSGA-II algorithm is based on the classification of all population individuals in layers called fronts. Individuals are compared in terms of no-domination, grouping all non-dominated individuals in the same front. For the sake of clarity, Pareto-optimal solutions are defined next:

Let $o_{1}, o_{2}, \ldots, o_{n}$ be objective functions which are to be maximized. Let $S$ be the set of all possible solutions. $s \in S$ is dominated by $t \in S$ (denoted $t \succ s)$ if $\exists j, j \in 1, \ldots, n$ such that $o_{j}(t)>o_{j}(s)$ and $\mathrm{i}, 1 \leq i \leq n, o_{j}(t) \geq o_{j}(s)$. A non-dominated solution is said to be Pareto optimal.

In our case, a solution, or network configuration, will dominate another if its coverage performance is better (i.e., provides a higher coverage level) and its cost performance is not worse (i.e., its network cost is not higher), or vice versa. It should be pointed out that a third objective could be easily introduced in the optimization problem, as for example the minimization of the interference produced to other networks.

Table 4.5 shows the pseudo-code of the algorithm. At each generation, $t$, the algorithm keeps two populations of the same size $n$ : the parent population $P_{t}$, and the child population $C_{t}$. The parent population stores the most fit individuals (the ones with the best cost and coverage performance) in each generation. The child population is obtained from the parent population after applying selection, crossover and mutation. As more than one parameter can be optimized per site, a hierarchical coding of the sites should be employed [63]. The first level of the hierarchy determines whether the site is active and the type of transmitter used. The second level determines for the transmitters the transmit power, whereas for the gap-fillers determines the transmit power, the transmitting antenna azimuth, and the emitter from which receive the signal. 
Table 4.5: Pseudo-code of the NSGA-II algorithm.

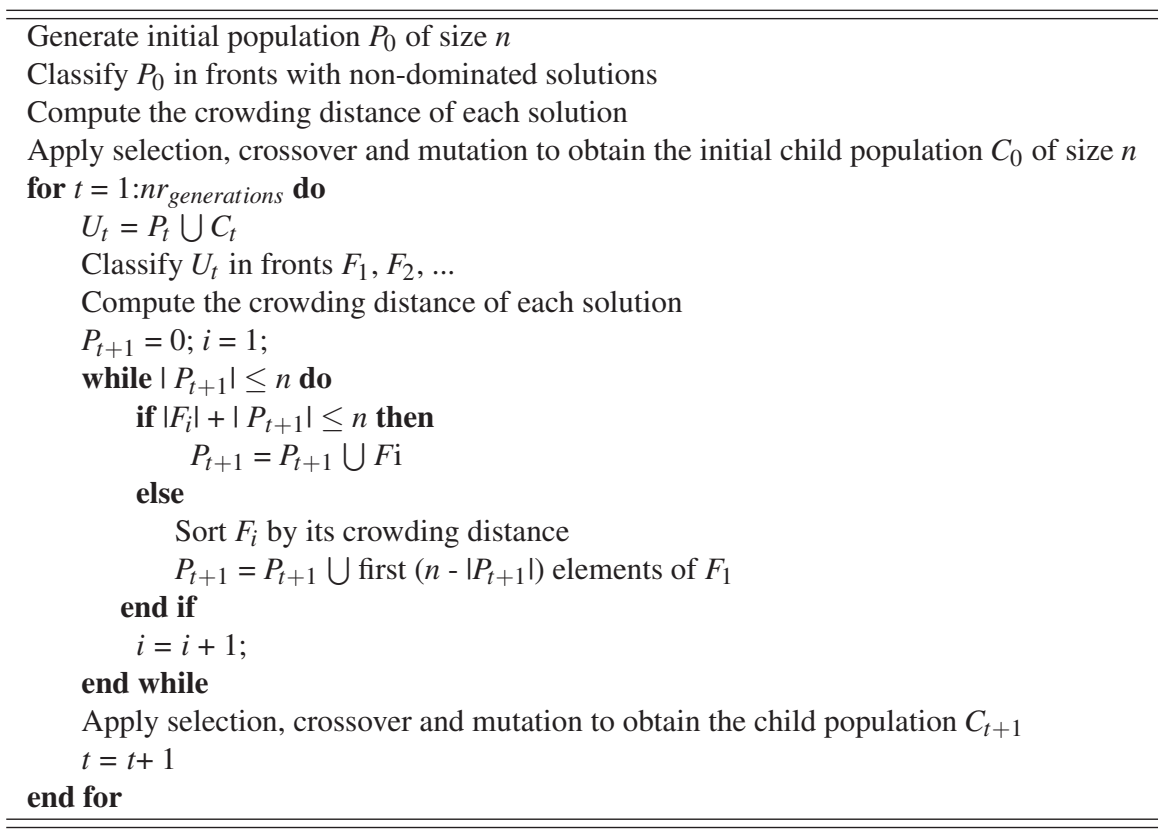

A parent is selected from two randomly chosen members from $P_{t}$ by means of a binary tournament. The member that wins the tournament is the one that belongs to a lower front, or has a higher crowding distance in case both belong to the same front. The crowding distance indicates the isolation degree of one particular individual in the solutions space. Individuals with a higher crowding distance are preferred in order to distribute the solutions along the Pareto-optimal front. The crossover operator used implies that child individual inherits some sites from one of his parents and the rest of the sites from the other parent. The crossing point is computed randomly. The mutation operator consists in changing randomly one optimization parameters of a randomly chosen site.

Once the child population is obtained, the algorithm works with the union set $U_{t}=$ $P_{t} \cup C_{t}$, selecting the $n$ most fit individuals to form the parent population of the next generation, $P_{t+1}$. First, all individuals of $U_{t}$ are classified in fronts. Sorted fronts are then included in $P_{t+1}$. If by including the front $F_{j}$ the desired size $n$ is exceeded, individuals of $F_{j}$ with a higher crowding distance are included in $P_{t+1}$. Application examples of the proposed network planning algorithm using only synchronized transmitters can be found in [40]. The proposed algorithm is especially suited for designing dense SFN in urban environments, where a large number of cellular sites might be available, and indoor and vehicular reception are required. 


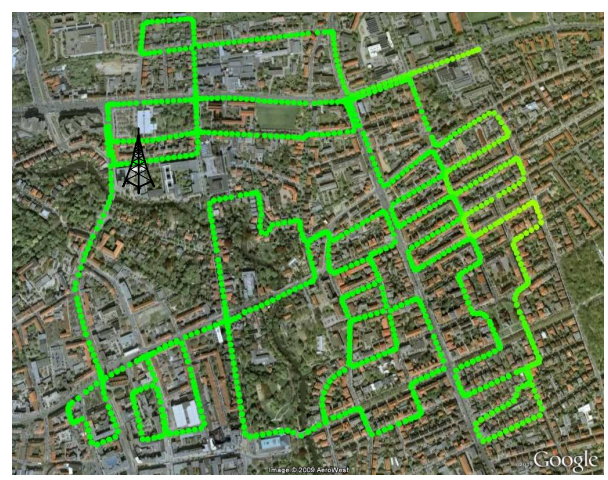

Figure 4.5: Map of Braunschweig with the position of the DVB-H transmitter and the considered streets in the simulations. Shown area is $2 \mathrm{~km} \times 2 \mathrm{~km}$.

\subsection{Coverage Estimation for Multi-Burst FEC Services}

Generally speaking, coverage estimation for wireless broadcast communication systems consists on predicting the service availability across a given area. In the particular case of DVB-H, the service availability at a certain location can be defined as the probability of correctly receiving a burst ${ }^{4}$. One location is considered as good covered if the average burst error rate is below 5\% [34].

For the conventional intra-burst MPE-FEC scheme, coverage estimation is a semi-static process due to the reduced burst duration of few hundreds of milliseconds. In a realistic scenario a coverage map can be easily derived directly mapping the signal availability in each a small area (coverage map grid resolution) into service availability level. This cannot be done with multi-burst FEC, as the probability of correctly receiving a burst depends on previous and/or future reception conditions. A valid approach for coverage estimation for multi-burst FEC is to perform dynamic system-level simulations, as described in Appendix A.2. They allow simulating a DVB-H network over time, being able to estimate the evolution of the transmission errors experienced by the users.

\subsubsection{Realistic Simulation Scenario}

The simulation scenario corresponds to the DVB-H pilot network of the University of Braunschweig (Germany), which consists of one transmitter at $57 \mathrm{~m}$ height with three sector antennas operating at $554 \mathrm{MHz}$, see Appendix A.1. Figure 4.4 shows a map of Braunschweig with the position of the transmitter and the streets considered. In the simulations the transmit power is set to $33 \mathrm{dBm}$ ERP (Effective Radiated Power) for each antenna to provide partial coverage over the service area.

\footnotetext{
${ }^{4}$ Although it is possible to receive busts partially, from a network planning point of view it is preferable to dimension for a maximum burst error rate, as not all applications may be able to use partially received bursts. Moreover users will experience different loss patterns. This approach is also worst-case, as the IP packet error rate is always lower.
} 

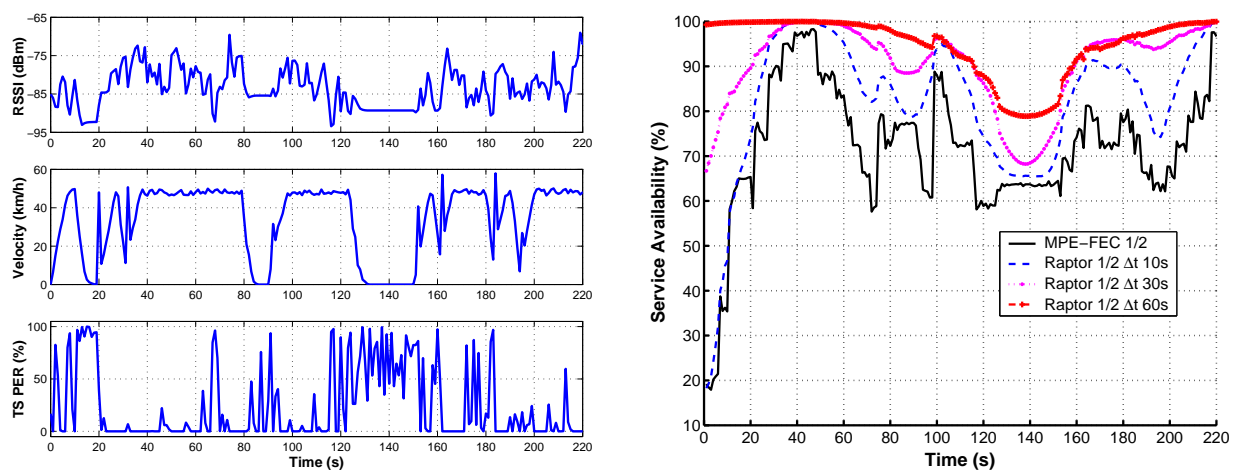

Figure 4.6: Example of simulated trajectory (left) and service availability with multi-burst FEC (right).

In the simulations we consider vehicular in-car users. The users' mobility has been simulated with the simulation tool SUMO (Simulation for Urban MObility) [133]. It employs street vector information and it takes into account traffic lights, turning probabilities, car densities etc., see Appendix A.2.1. Signal level variations have been simulated taking into account path loss, correlated shadowing and fast fading. The path loss has been obtained using the macro cell propagation model proposed in [134] using terrain height and clutter data with $50 \mathrm{~m}$ resolution. Shadowing is implemented by a lognormal distribution with a standard deviation of $5.5 \mathrm{~dB}$ and a correlation distance of $20 \mathrm{~m}$. The spatial correlation follows a first-order exponential model. Fast fading is included in the DVB-H physical layer performance model within the TU6 channel model described in Appendix A.3. The mode considered is: FFT 8K GI 1/4 16-QAM 1/2. The cycle time between bursts is set to one second, and the burst size is adjusted to achieve a constant data rate of $384 \mathrm{~kb} / \mathrm{s}$. The multi-burst FEC latency is thus the number of bursts jointly encoded (encoding period) in seconds.

\subsubsection{Coverage Estimation Example}

Figure 4.5 shows an example of the received signal level (RSSI), velocity and TS packet error rate at the physical layer of one simulated trajectory, and the service availability for multi-burst FEC $1 / 2$ and different latencies. The reference case with MPE-FEC $1 / 2$ is also shown for comparison. Results have been obtained performing multiple realizations of the fading processes (shadowing and fast fading) with different random seeds ${ }^{5}$. That is, the velocity and the path loss remains the same, but the signal strength varies due to different shadowing. If the user stops, the shadowing is constant, but there is also variability due to fast fading.

\footnotetext{
${ }^{5}$ When modeling the wireless radio channel as three multiplicative processes with different time-scales (path loss, shadowing and fast fading) [121], the mean path loss is considered to be deterministic, whereas fading is a statistical process. To achieve statistically consistent results, 10.000 fading iterations have been performed.
} 


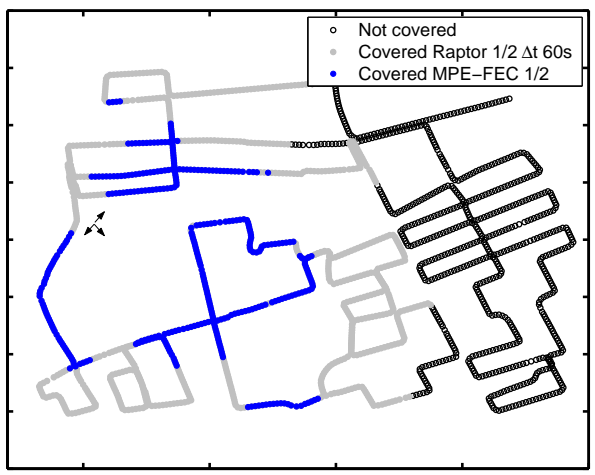

(a) Service coverage map.

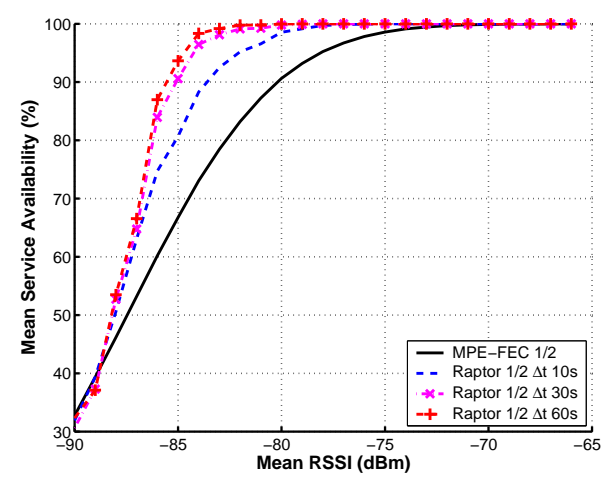

(b) Service availability vs. received signal level.

Figure 4.7: Coverage estimation example with multi-burst FEC. Constant velocity $36 \mathrm{~km} / \mathrm{h}$.

In the figure it can be seen than the code rate is robust enough such that service availability increases for larger encoding periods. But more importantly here, shown results can be directly translated into space, and thus a service coverage map can be plotted. It is worth to mention that results are valid for both forward and backwards directions, but they are specific for the particular velocity trace considered. Therefore, to predict the service availability with multi-burst FEC it is necessary to accurately estimate both received signal strength and mobility pattern.

In order to evaluate the service coverage in the complete scenario under study, a trace has been simulated that enters all streets. Figure 4.7(a) shows a coverage map for MPE-FEC 1/2 and for multi-burst FEC 1/2 with 60 seconds latency for a constant speed of $36 \mathrm{~km} / \mathrm{h}$. The simulation time is about 28 minutes. In the figure green dots represent locations with a service availability with MPE-FEC equal to $95 \%$ or higher, whereas black and not filled dots represent locations with a service availability below 95\%. Black dotted locations are additionally covered with the target probability for multi-burst FEC.

In order to quantify the coverage gain, we compute the mean service availability as a function of the mean received signal strength for different encoding periods, see Figure 4.7(b). It can be seen that for the RSSI range considered the service availability increases with the encoding period. The coverage gain is the reduction in the required RSSI threshold to achieve 95\% service availability. For MPE-FEC a received signal level of $-78.1 \mathrm{dBm}$ is necessary. For multi-burst FEC with 60 seconds latency the signal strength threshold is reduced down to $-84.7 \mathrm{dBm}$. The difference between these RSSI values can be interpreted as a link margin gain $(6.6 \mathrm{~dB}$ in this case $)$.

In Figure 4.7(b) we can also note that multi-burst FEC does not only yield a gain in coverage, but also a gain in service quality in locations covered with MPE-FEC. For instance, for 10 seconds latency the service availability is close to $100 \%$ when the reference case is only $90 \%$. In this case we have a clear gain in service quality, but also in coverage (about $4 \mathrm{~dB}$ link margin). This gain is is achieved between the RSSI range where the service availability for MPE-FEC lies between $95 \%$ and $100 \%$. 


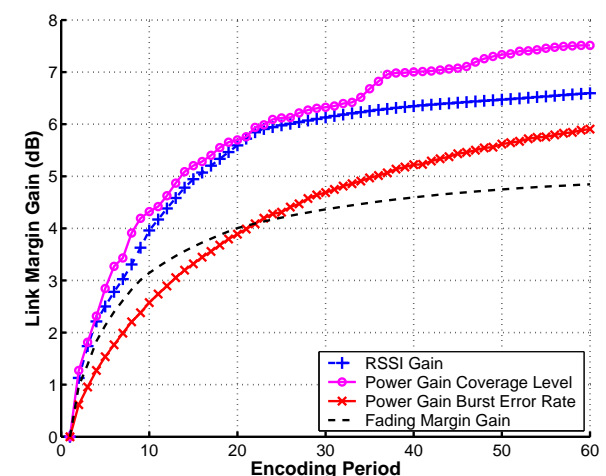

(a) Comparison link margin gains. Constant velocity $36 \mathrm{~km} / \mathrm{h}$.

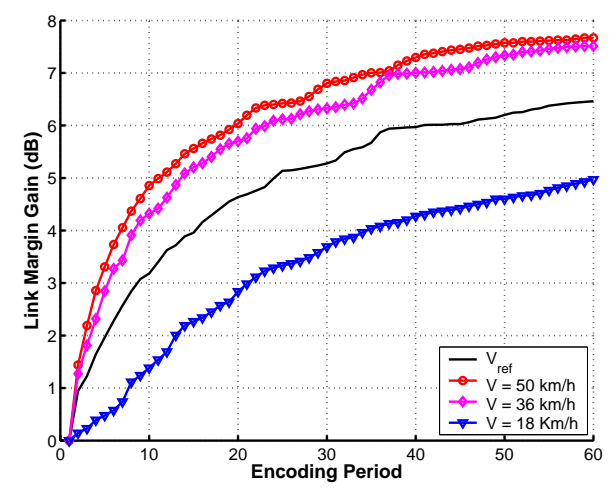

(b) Effect of variable velocity. Power gain coverage level.

Figure 4.8: Link margin gain with multi-burst FEC 1/2 vs. Encoding period.

Obviously, there is no gain in areas where there is full coverage for MPE-FEC as there are no transmission errors to correct. In areas with very poor signal level there is no gain either (not shown in the figure), because in this case the total amount of errors is larger than the error correction capability. In our example this occurs in the right-side part of the scenario. Therefore, for a given trajectory and service area the gain depends on the transmitted power.

\subsubsection{Comparison Link Margin Gains}

From a coverage estimation point of view, we have seen that given an existing network and service area, multi-burst FEC yields a link margin gain in terms of reduced RSSI threshold to achieve a target service availability. From a network planning (dimensioning) point of view, the gain can be expressed in terms of a reduction in the transmission power required to achieve a target coverage level over the service area. The coverage level can be defined for example as the percentage of locations which service availability exceeds $95 \%{ }^{6}$.

Figure 4.8(a) compares both link margin gains for multi-burst FEC 1/2 as a function of the encoding period. It can be observed that the gains are rather similar. However, gain values are between $1 \mathrm{~dB}$ and $2 \mathrm{~dB}$ larger than the fading margin gain for 5\% burst error rate considering only fast fading and shadowing (result from Chapter 3 , also pictured in the figure together with the gain in reduced transmit power to achieve the same percentage of erroneous bursts across the trajectory). The difference between these two curves is the path loss variability. We can see that for low encoding periods the gain with path loss is smaller, whereas for large encoding periods the gain is higher.

\footnotetext{
${ }^{6}$ Another possibility would be to dimension the network taking into account the overall QoS perceived by the users using system-level simulations. This approach is considered in the next chapter.
} 
Multi-burst FEC can cope not only against fast fading and shadowing, but also against path loss if the protection period is large enough and if the code rate is robust enough. Compared to the previous chapter, where only fading was considered, the path loss variability increases the time diversity of the mobile channel, and hence the maximum potential gain that can be achieved with multi-burst FEC increases. However, signal level variations due to path loss are noticeably slower than the variations due to fading. Hence, path loss may reduce the gain due to fading for low encoding periods as shown in the figure. Indeed, the path loss determines the areas where there is no gain because the signal level is either too high or too low. For a given network the highest gain occurs at the edge of the coverage area for the reference intra-burst FEC case, as shown in Figure 4.7(a). In this sense, the distribution of the outage areas within the service area becomes relevant. The case with one single transmitter is the worst scenario. Higher gains for small and moderate latencies can be expected in dense SFN scenarios because outage areas are much more uniformly distributed.

Finally, we investigate the effect of having a variable velocity with the possibility that users stop due to traffic light. We can expect the gain to be reduced, especially if the user stops in a spot with a reduced coverage. In this case very long latencies will be required to achieve significant gains, as the user will suffer long outage periods. Assuming a code rate $1 / 2$, for example, it would be in general needed to consider latencies at least twice the stop time to cope with such outages.

Figure 4.8(b) shows the link margin gain for the simulated trace depicted in Figure 4.5 for different constant velocity values, and for the reference velocity provided by SUMO. The average velocity across the trajectory is $36 \mathrm{~km} / \mathrm{h}$, with a maximum velocity of $50 \mathrm{~km} / \mathrm{h}$ and about 3 minutes and a half waiting time due to traffic lights (average waiting period lies between 20 and 30 seconds). Figure 4.6 shows an example of the simulated velocity across one street. Results obtained show that the gain is reduced compared to the case of having a constant velocity, but the reduction is not very significant. In this particular example the penalization of considering a variable velocity with periods without movement is lower than reducing the average velocity by half.

\subsection{Conclusions}

In this chapter we have shown that with MPE-FEC very dense SFNs with a large number of sites are required to provide very high DVB-H capacity and nearly full coverage, especially to achieve indoor and vehicular in-car coverage. Moreover, it is usually very costly to guarantee coverage to the last few per cent of the worst-served locations. This penalty is particularly evident for area coverage targets over $90 \%$ of service area locations. One solution to reduce the cost of covering the final percentages of locations may be to use gap-fillers (on-channel repeaters), instead of synchronized transmitters. On the other hand, good scalability figures in capacity, up to the envisaged $10 \mathrm{Mb} / \mathrm{s}$ commercial requirement, can be achieved with an infrastructure cost per $\mathrm{Mb} / \mathrm{s}$ approximately constant. 
In this chapter we have also presented a methodology to estimate the coverage for multi-burst FEC services in DVB-H networks based on dynamic system-level simulations. Multi-burst FEC requires dynamic analysis over time to take into account the mobility of the users, because the probability of correctly receiving a burst depends on previous and/or future reception conditions. The gain is not constant across the service area, and it cannot be directly included in the link budget for coverage estimation. Only for relatively short latencies, such as the required by mobile TV services, and assuming a constant velocity it may be possible to express the gain as a reduction of the fading margin required to cope with fast fading and shadowing. For moderate/large latencies and high user speeds it is necessary to accurately estimate both received signal strength and mobility pattern (user velocity and trajectory) to predict the service coverage with multi-burst FEC in a realistic scenario.

Our results show that the gain obtained by performing a multi-burst protection of the transmission in an existing DVB-H network is twofold, as the area coverage is enlarged, but also the service quality perceived by the users is enhanced with the same infrastructure. Moreover, we have shown that for vehicular users multi-burst FEC can also yield an additional gain due to path loss compared to the fading margin gains obtained in Chapter 3. The level of latencies required to benefit from such variability will heavily depend on the distribution of the outage areas across the service area and the positions where users stop. Very large latencies will be required if users become static in bad covered spots. In this sense, the worst scenario is the single transmitter case. Higher gains will in general hold in dense SFN scenarios because the outage areas are usually much more uniformly distributed. 



\section{Chapter 5}

\section{Radio Resource Management in Hybrid Cellular and DVB-H Systems}

In the previous chapters we have seen that multi-burst FEC can improve significantly the DVB-H transmission robustness for both streaming and file download services, such that a more cost efficient provisioning of mass mobile multimedia services can be achieved. The reason is that thanks to the mobility of the users it is possible to provide reliable delivery in networks with partial coverage over the service areas by transmitting a sufficiently large amount of repair data with a sufficiently long protection period.

In this chapter we investigate the potential cost delivery savings that can be achieved if an evolved 3G+ cellular network with HSDPA and MBMS is used to repair errors of the broadcast transmissions of a DVB-H network. In a realistic scenario, there may be users that experience significantly worse DVB-H reception conditions than the majority, such that it may be more efficient to serve them through the cellular network. We focus on file download services because they exhibit the largest potential from a hybrid cellularbroadcast delivery point of view. File delivery requires an error-free reception of the files, which cannot be guaranteed for each and every user after a DVB-H transmission because some users might have experienced too bad reception conditions. Moreover, it does not have very stringent time constraints such as streaming delivery. To enable an easy and efficient implementation of the repair mechanisms, we adopt the use of forward error correction at the application layer (AL-FEC) with Raptor coding [38], see Chapter 2.4. With AL-FEC specific source packets are not required to be retransmitted, and repair transmissions consist of additional parity packets that can be used for all users. Hence, repair data can be efficiently delivery with p-t-m broadcast/multicast transmissions with DVB-H or MBMS. AL-FEC is currently standardized for file delivery in MBMS as mandatory [56], whereas in IP datacast its use is optional [54]. 
We assume a hybrid DVB-H/3G+ system in place, with the DVB-H network providing only partial coverage over the service area. We adopt the perspective of a network operator which controls both cellular and broadcasting networks. This approach is to some extent equivalent to a service provider that leases capacity from both radio access broadcasting networks for a price that can vary over time. We adopt a similar framework for cost-based RRM in hybrid cellular and broadcast systems than the originally proposed in [71]. The delivery cost is assumed to be proportional to the amount of radio resources employed. This cost model also accounts for the opportunistic or unfavorable situations that may arise in real life implementations, where prices for radio access are not only depending on the production costs but also on the alternative use of the resources. In our case, the broadcast cost is proportional to the amount of data transmitted in DVB-H, and the cellular cost is proportional to the transmission energy, defined as the product of the transmit power times the transmission time. Minimizing the transmission energy the number of services that can be provided with the same amount of power and during the same time period is maximized. Under full background interference conditions, the transmission energy is inversely proportional to the cell capacity.

It should be pointed out that we investigate optimum delivery configurations to achieve a target file acquisition probability (percentage of users that successfully receive the file). Transmissions are configured statistically using dynamic system-level simulations in a realistic scenario. Therefore, we do not try to solve the problem of predicting the optimum configurations beforehand. This research topic is left for future work.

The chapter is structured as follows. We first introduce the simulation scenario in Section 5.1. In Section 5.2 we analyze multicast file delivery in evolved 3G+ networks with HSDPA and MBMS. We study under which conditions MBMS with a single p-t-m transmission is more efficient than HSDPA with multiple p-t-p transmissions (one per active user). We also investigate the capacity increase that can be achieved using HSDPA for error repair of the MBMS transmissions. In Section 5.3 we review filecasting in DVB-H networks from an RRM perspective. In Section 5.4 we analyze the case when HSDPA and MBMS are used for error repair of the DVB-H transmission in a hybrid DVB-H/3G+ system. The chapter concludes with a summary of key results in Section 5.4.

\subsection{Simulation Scenario}

The DVB-H simulation scenario corresponds to the DVB-H pilot network of the University of Turku (Finland), which consists of two transmitters operating at $610 \mathrm{MHz}$. The network is dimensioned for pedestrian outdoor reception. Figure 5.1 shows a map of Turku with the position of the transmitters and the service area considered. For the cellular network a synthetic scenario has been considered. There are 24 sites uniformly distributed over the service area shown in Figure 5.1. Omnidirectional antennas have been assumed at the base stations. The cell radius is $866 \mathrm{~m}$, which yields an inter-site distance of $1.5 \mathrm{~km}$ and a cell area of $1.6 \mathrm{~km}^{2}$. For a detailed description of the scenario and the models employed see Appendix A. 


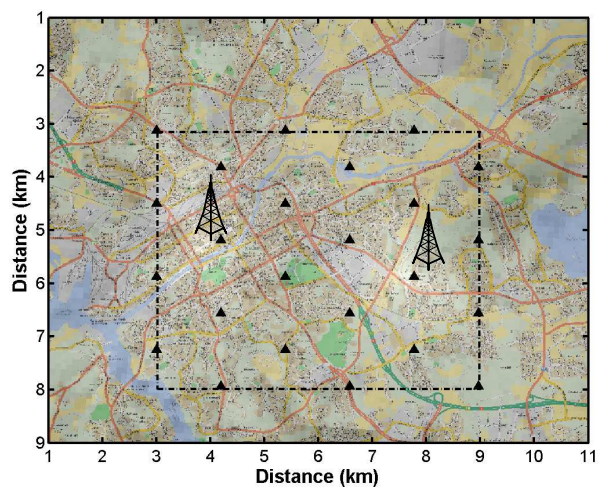

Figure 5.1: Map of Turku with the positions of the DVB-H transmitters and the simulated cellular base stations.

In our simulations, 10.000 users are initially uniformly distributed within the central part of Turku. This allows for a 1\% confidence interval for the results obtained in terms of file acquisition probability. They do not leave the service area but bounce at the edges. Vehicular users move according to the statistical mobility model for in-car users in urban environments described in Appendix A.2.1 [135]. Pedestrian users move with a random walk with a constant speed of $3 \mathrm{~km} / \mathrm{h}$.

\subsection{Filecasting in 3G Cellular Networks with MBMS and HSPDA}

\subsubsection{File Delivery in MBMS}

Generally speaking, a file download service in MBMS consists in three phases:

1. Service advertisement phase; in which the service is announced and set-up by the network and the users discover the service.

2. Initial MBMS file transmission phase; in which both source data file and a fixed pre-configured amount of Raptor parity data are initially transmitted with a p-t-m connection.

3. Post-delivery repair phase; in which users not able to decode the file after the initial MBMS transmission are served by default via p-t-p (HSDPA) connections to complete the reception of the file. In case too many users fail to receive the file it is also possible to employ a $\mathrm{p}-\mathrm{t}-\mathrm{m}$ connection.

Next, we discuss the initial MBMS file transmission phase and the error repair phase from an overall radio resource management perspective. In order to achieve an efficient delivery it is needed to optimize the initial MBMS file transmission including the trade-off with the error repair phase. Figure 5.2 shows a temporal diagram of both phases. For a more detailed description of the procedures involved we refer to [56]. 


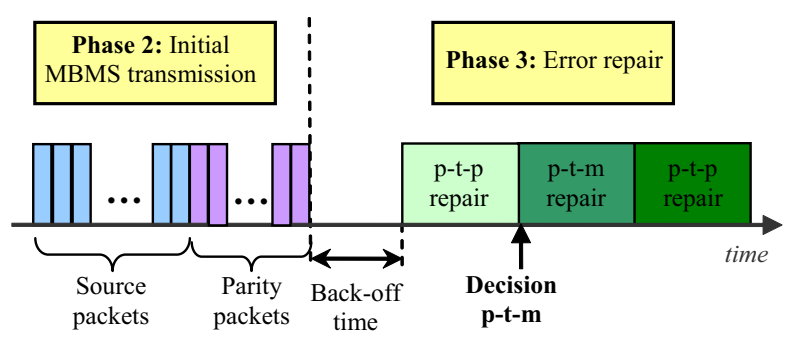

Figure 5.2: MBMS file download service. Initial MBMS transmission and repair phase.

\section{Initial MBMS File Transmission Phase}

In this phase the MBMS server must configure the transmission parameters statistically to ensure that the file is successfully received by the worst-case user contemplated, such that the desired file acquisition probability is reached (percentage of users that receive the file).

The robustness of the MBMS transmission is mainly given by the coverage level and the amount of Raptor parity data transmitted. The coverage level depends on many factors: scenario, macro diversity technique implemented in the terminals, interference conditions, etc. The only parameters that the network operator can control are the transmit power, the radio bearer settings, and the amount of Raptor parity data transmitted. The transmit power and the radio bearer yield the area coverage. Obviously, the higher the transmit power, the larger the coverage level (keeping the bearer fixed), and thus the amount of required parity data will be smaller, and the service transmission time will be reduced. However, maximizing the transmit power does not necessarily need to be the optimum configuration because $3 \mathrm{G}$ systems are interference-limited, and the interference level is directly proportional to the transmission powers. Moreover, a very high coverage level does not exploit the diversity gain that can be achieved with Raptor coding due to the mobility of the users. This diversity gain is higher for larger user velocities, and thus the amount required of parity data depends on the degree of mobility of the users [76]. On the other hand, when increasing the bearer data rate the file should be in theory received faster by the users. However, it should be taken into account that an increase in the bearer data rate results in a reduction of the coverage level (keeping the transmit power constant), what implies an increase in the transmission time.

\section{File Repair Phase}

The purpose of this phase is to repair erroneous received files after the initial MBMS transmission. Terminals not able to recover the file notify the minimum set of data packets required to repair the file or simply the total number of correctly received packets [56]. To avoid congestion, error reporting messages from terminals can be distributed over time within a back-off window and across multiple repair servers. The back-off window should be large enough to prevent congestion, but should not unnecessarily increase the duration of the repair phase. 
As mentioned before, terminals start the repair phase using dedicated p-t-p HSDPA connections, but if the number of active users in this phase is high enough it is possible to employ a p-t-m connection with MBMS. However, during the initial file transmission there is no communication between the terminals and the server. Therefore, once the MBMS transmission is finished the server does not have any information about the number of users that have not received the file and the amount of repair data needed by each of them. This information can be estimated in the beginning of the p-t-p repair session.

The decision of performing a p-t-m repair transmission should be taken as soon as possible once a representative number of error reporting messages have been collected. Usually, it is recommended to take this decision once the $10 \%$ of the back-off window has elapsed [78]. The amount of repair data transmitted through the p-t-m connection can be for example the maximum amount of repair data requested by the users at that time. Once the p-t-m repair session is completed, a new p-t-p repair session can be initiated if needed as there is no guarantee that users will receive the data transmitted with MBMS. In this case the length of the second back-off window should be smaller than the first one, since a lower load on the repair server can be expected. It should be also pointed out that the repair server should immediately proceed to initiate a p-t-m repair session as soon as congestion is detected. This may happen if, for example, the robustness of the initial MBMS transmission is too low, or unexpected transmission errors occur. According to [78], the link most likely to become the bottleneck is the one between the repair server and the cellular network (more precisely the gateway GPRS support node, GGSN).

In addition to the duration of the back-off window, the main parameter that the network operator should configure in the p-t-p repair session is the HSDPA transmit power devoted to repair the file. Higher power levels will imply that the users receive the file faster, although it may not be the most efficient resource allocation as we have explained before. It should be noted that the performance of the p-t-p repair phase with HSDPA depends on the actual number of users that fail to receive the file after the initial MBMS transmission and their positions.

\subsubsection{Performance Evaluation}

In this section we present simulation results for a file download service using only MBMS with a single $\mathrm{p}-\mathrm{t}-\mathrm{m}$ transmission, using only HSDPA with simultaneous $\mathrm{p}-\mathrm{t}-\mathrm{p}$ transmissions, and using MBMS and HSDPA jointly [88]. Results focus on determining the optimum transmission configurations that provide the minimum transmission energy to achieve a given file acquisition probability target for a given number of users per cell ${ }^{1}$ within a given time (for the cases where the transmission of the file takes place over a limited time period). The transmission energy is defined as the product of the transmit power, $P_{t x}$, and the active transmission time, $T_{t x}$ :

$$
E=P_{t x} \cdot T_{t x} .
$$

\footnotetext{
${ }^{1}$ The performance of HSDPA depends on the number of active users per cell.
} 
By minimizing the energy, the radio resource usage is minimized, and the cell capacity is maximized. The cell capacity represents a measure of the total data rate that can be provided when all the available power is used for file download services. It can be computed as:

$$
C_{\text {cell }}=\frac{P_{\text {available }}}{P_{t x}} \cdot \frac{F_{s}}{T_{t x}}
$$

where $P_{\text {available }}$ is the available power, $P_{t x}$ is the transmit power, $F_{s}$ is the file size and $T_{t x}$ the transmission time. Note how the cell capacity is inversely proportional to the transmission energy, but this only applies for a scenario with constant load conditions.

For MBMS we investigate the optimum transmit power and bearer data rate. The effective bearer data rate depends on several factors (such as spreading factor, turbo-code code rate, transport block format, etc.), and thus several configurations may provide the same data rate. The reference MBMS radio bearers that have been defined for interoperability and testing purposes as preferred configurations for specific data rates can be found in [136]. We have analyzed the reference case without any macro diversity combining technique.

For HSDPA we only investigate the optimum transmission power, as we assume a MaxCIR scheduling algorithm. This strategy consists of serving the user who presents the best reception conditions, or equivalently, the one with the highest achievable data rate.

For the hybrid MBMS-HSDPA delivery case we investigate the optimum balance between the initial MBMS transmission and the HSDPA error repair phase by computing the optimum file acquisition probability after the MBMS transmission. It can be shown that the optimum delivery configuration corresponds to a hybrid multicast-unicast delivery with only p-t-p repair transmissions, as in case a p-t-m repair transmission is needed it would have been more efficient to increase the duration of the initial $\mathrm{p}-\mathrm{t}-\mathrm{m}$ transmission instead. The overall energy subject to minimization is thus:

$$
E_{\text {Hybrid }}=E_{M B M S}+E_{H S P D A} .
$$

We assume that the same power employed for MBMS is used for HSDPA, and that the back-off window during the error repair phase is set to zero, in order to make a fair comparison with the results obtained when using only MBMS. This implies that there are no congestion problems in the network, and that the radio link is the bottleneck.

As a reference study case we will consider a $16 \mathrm{Mb}$ file and a $99 \%$ target file acquisition probability, although the implications of considering smaller and larger file sizes, and different acquisition probability targets will be also discussed. Vehicular users are considered in the cellular simulations. More results can be found in [88]. Feasible MBMS bearer data rates employed in the simulations are: $64,96,128,192$, and $256 \mathrm{~kb} / \mathrm{s}$, and useful transmit power values range from 0.5 up to $16 \mathrm{~W}$ for both MBMS and HSDPA. In the simulations it has been assumed an interference scenario with full background load conditions where all base stations transmit at their maximum power. It has been considered that control channels use $20 \%$ out of the maximum total transmission power of $20 \mathrm{~W}$, and thus capacity figures are for an available power of $16 \mathrm{~W}$. 


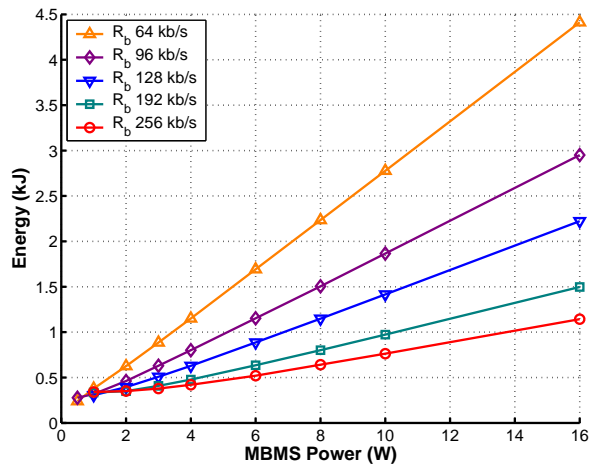

(a) Cell transmission energy vs. Transmit power.

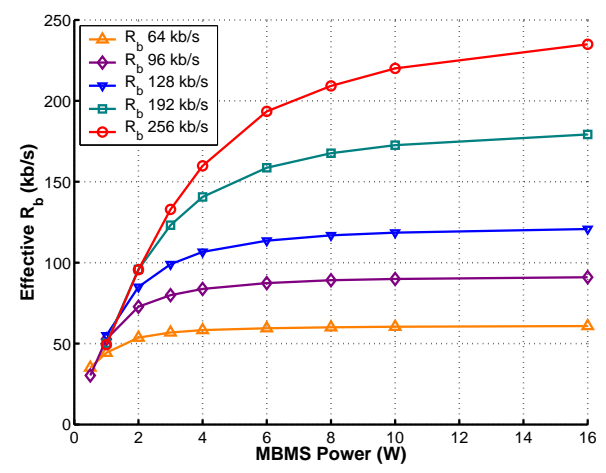

(b) $R_{b, e f f}$ vs. Transmit power.

Figure 5.3: MBMS file download results without time constraints. $F_{s}=16 \mathrm{Mb}$. Acq. prob. $99 \%$.

\subsubsection{File Delivery Results with MBMS}

Figure 5.3(a) shows the energy required per cell to achieve a $99 \%$ acquisition probability of a $16 \mathrm{Mb}$ file as a function of the transmit power for different bearer data rates for the reference case without macro diversity. We can see that there is an optimum power value at low powers that minimizes the energy for a given bearer data rate. Results can be explained due to the fact that it is more efficient to allow relatively high loss rates at the physical layer and correct them at the application layer in order to take advantage of the spatial diversity introduced by the mobility of the users [76]. This implies transmitting important amounts of Raptor parity data. For high transmit powers the coverage level is close to $100 \%$, and thus almost no Raptor parity data is required. For this reason the diversity gain is smaller if macro diversity techniques are considered, because they improve the coverage level significantly [11]. The Raptor coding efficiency also improves for larger files. The reason is that the time diversity experienced by the users during the transmission of the file is larger for a given proportion of parity data transmitted.

Figure 5.3(b) shows the effective data rate for each combination considered, defined as the ratio between the file size, $F_{S}$, and the transmission time required to achieve the target acquisition probability, $T_{t x}$ :

$$
R_{b, e f f}=\frac{F_{s}}{T_{t x}} .
$$

As expected, the larger the useful transmit power, the higher the effective data rate, achieving a faster distribution of the file.

Coming back to Figure 5.3(a), we can notice the importance of optimizing the bearer data rate as a function of the transmit power employed. In the figure it can be observed that the optimum bearer for large powers is the maximum considered, $256 \mathrm{~kb} / \mathrm{s}$. The reason is that the coverage level is high enough such that most users can benefit of transmitting more data per TTI. When the transmit power (and coverage level) decreases, the optimum bearer data rate is reduced. 


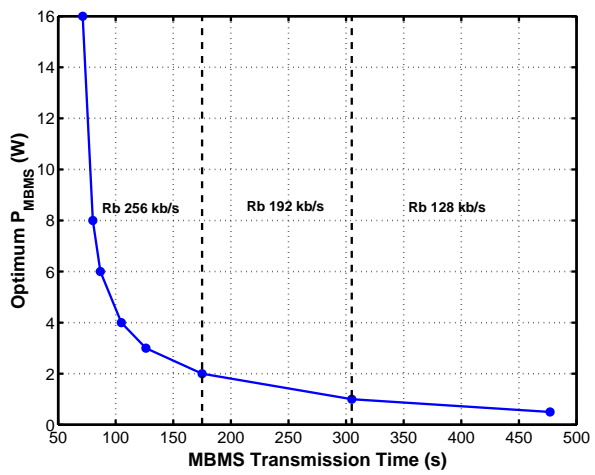

(a) Optimum power and bearer vs. Time.

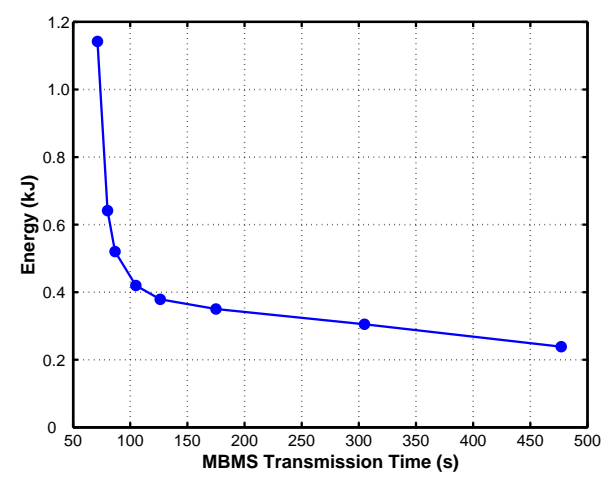

(b) Minimum cell energy vs. Transmission time.

Figure 5.4: Time-constrained MBMS file download results. $F_{S}=16 \mathrm{Mb}$. Acq. prob. $99 \%$.

We can see this effect in Figure 5.4(a), that shows the optimum transmit power as a function of the transmission time highlighting the ranges where each bearer data rate is optimum. Notice that the fastest delivery is always achieved for the largest transmit power and bearer data rate considered. Figure 5.4(b) depicts the minimum energy as a function of the transmission time. The trade-off between minimizing the resource consumption and the transmission time of the files is evident. The relationship between energy and transmission time shown reveals that the once certain duration is exceeded the energy reduction is noticeably slower. Hence, the preferred transmission configurations may not be the ones that provide the minimum energy to avoid very long transmission times.

Results obtained agree with the ones presented in [76], where the code rate of the turbo-coder at the physical layer is optimized. However, it should be pointed out that there is a performance degradation when puncturing the turbo code rate, and it may be more efficient to change other bearer parameters like the spreading factor instead [136]. It should be pointed out that macro diversity combining techniques generally implies higher optimum bearer data rates, because the coverage level is higher. On the other hand, the optimum bearer data rate decreases as a function of the file acquisition probability target, because the coverage level perceived by the worst-case user served (which gives the final energy value) is reduced.

\subsubsection{File Delivery Results with HSDPA}

Multicast delivery with multiple p-t-m HSDPA simultaneous connections yields a transmission energy directly proportional to the number of active users. Interestingly, the minimum energy is achieved for the minimum power considered like in MBMS, which also incurs the largest delivery time. Therefore, there is again a trade-off between resource consumption and service transmission time. 


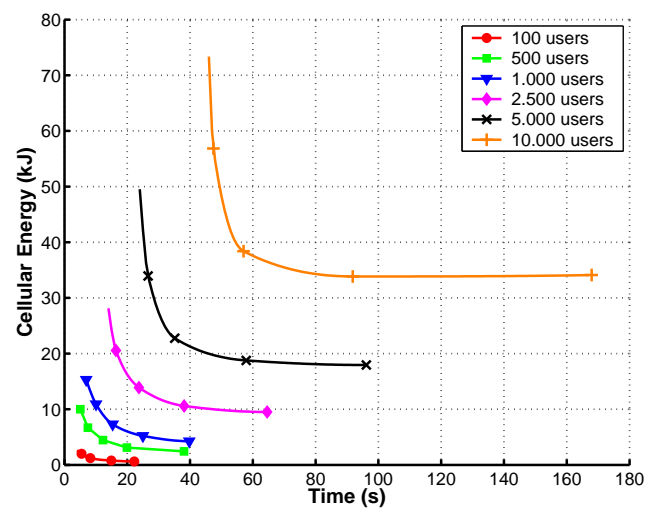

Figure 5.5: HSDPA file download results with time constraints. Total transmission energy vs. transmission time. $F_{S}=16 \mathrm{Mb}$. Acquisition probability $99 \%$.

Figure 5.5 shows the total cellular energy required to deliver a $16 \mathrm{Mb}$ file to $99 \%$ of the users with HSDPA in the scenario of Turku as a function of the transmission time for different number of users in the service area. Recall that there are 24 cellular sites in the service area.

If we compare the energy required with HSDPA as a function of the number of active users with the energy required with MBMS for $2 \mathrm{~W}$ transmitted power (this value represents a good trade-off between resource efficiency and the repair time), it is more efficient to employ HSDPA if there are up to 1.150 users in the service area (about 24 users per cell). The threshold depends on the file size and the acquisition probability target. For larger files the threshold decreases because MBMS performs more efficiently. HSDPA also benefits of larger files in the sense that there is a larger scheduling gain, but in a lower extent than MBMS. In contrast, the threshold increases for larger acquisition probabilities targets. This is because it becomes increasingly more costly to serve the final percentage of users using only MBMS compared to HSDPA. These users are the ones located in bad reception locations that move at low speeds, and they cannot be efficiently served with MBMS if low transmit powers are employed. The key with HSDPA is that the transmissions are optimized for each user individually, and that resources are only consumed when one user is actively using the service (i.e., there is at least one user in coverage). With MBMS the transmission parameters are fixed is and the transmission remains active during the complete initial file transmission phase (even if all users receive the file).

It is worth highlighting that the threshold does not increase if larger HSDPA data rates are considered. The interference level is so high and the useful transmission power is so low that it is not possible to benefit from higher rates. This may be useful for a timeconstrained file delivery in a network with low/medium load conditions. With HSDPA, if there is a maximum time to deliver the file, the optimum transmit power is the minimum value that transmits the file in due time. However, the efficiency of MBMS compared to HSDPA increases when reducing the time to deliver the file to the users as it is more difficult to serve them with simultaneous p-t-p connections. 


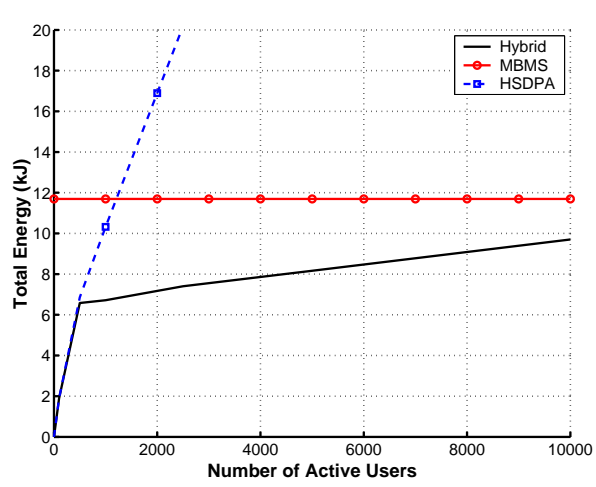

(a) Minimum energy.

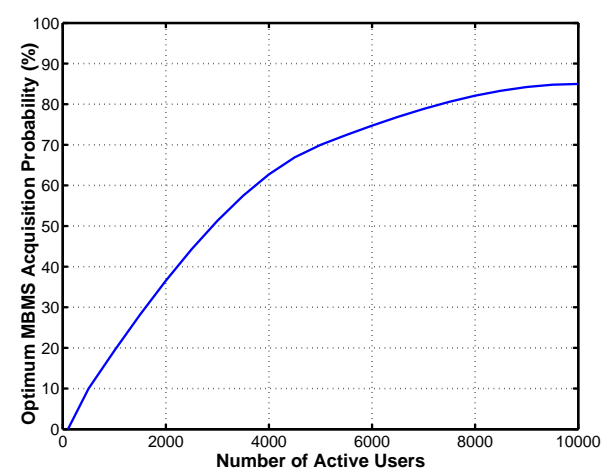

(b) Optimum MBMS acquisition probability.

Figure 5.6: Hybrid MBMS and HSDPA file download results for a $99 \%$ acquisition probability of a $16 \mathrm{Mb}$ file as a function of the number of active users in the system.

\subsubsection{File Delivery Results with MBMS and HSPDA}

Figure 5.6(a) shows the minimum energy that can be achieved with a hybrid MBMSHSDPA delivery to obtain a $99 \%$ acquisition probability of a $16 \mathrm{Mb}$ file as a function of the number of users in the system. The cases using MBMS and HSDPA separately are also shown for comparison. The transmit power value employed for both MBMS and HSDPA is $2 \mathrm{~W}$.

The only parameter subject to optimization for the hybrid delivery is the MBMS transmission time, or in other words the percentage of users that successfully receive the file during the MBMS transmission. The optimum MBMS acquisition probability increases as a function of the number of active users, see Figure 5.6(b). This is simply because the efficiency of MBMS compared to HSDPA increases with the number of users. For few users, up to 100 users in in our example, it equals to zero percent; meaning that all users are served with HSDPA, and that there is no gain with the hybrid delivery. For more users than this threshold, there is a potential energy reduction that can be achieved using MBMS and HSDPA jointly. The gain increases until the crossing point of the two reference curves using HSDPA and MBMS separately. This is the point where the highest energy reduction with the hybrid delivery is achieved (about $40 \%$ energy reduction in this case).

The gain brought by the hybrid delivery increases for higher acquisition probabilities targets, as it becomes very costly to serve the last percentage of users using only MBMS. The energy reduction achieved behaves in a similar way as the number of users required so MBMS is more efficient than HSDPA. That is, the gain decreases for larger files and when implementing macro diversity techniques. The reason is that in these cases MBMS performs better, and thus the gain obtained by using HSDPA as a complement is lower. 


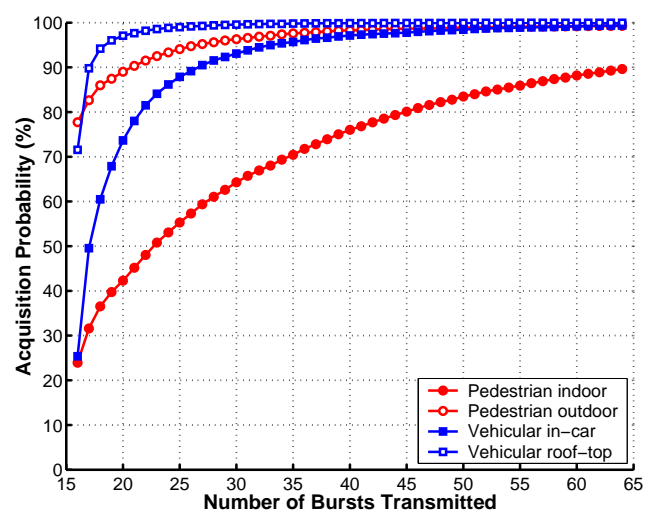

Figure 5.7: Acquisition probability of a $16 \mathrm{Mb}$ file vs. number of bursts transmitted for different reception cases.

\subsection{Filecasting in DVB-H Networks}

In this section we address radio resource management aspects for filecasting services with AL-FEC in DVB-H networks. We present results on the performance of the initial DVB-H transmission and considerations that should be taken into account when designing the postdelivery repair phase with a cellular network. We compute the percentage of users able to decode the entire file (acquisition probability) as a function of the number of bursts transmitted and the amount of necessary repair data needed by the rest of the users that do not manage to decode the file. Similar performance evaluation results comparing the performance of AL-FEC with MPE-FEC can be found in [89].

The transmission parameters that the network operator has to configure are the cycle time between bursts, the burst size, and the amount of parity data transmitted (code rate or FEC overhead). In Chapter 3 we have seen that for AL-FEC the performance improves increasing the cycle time, at the expense of a larger delivery time. One the cycle time exceeds certain duration, such that there is little correlation between reception conditions of consecutive bursts, there is no further gain by increasing the cycle time. Regarding the burst size, the performance improves slightly for smaller data bursts (assuming a constant download data), at the expense of larger power consumption at the terminals. However, the amount of parity data transmitted is ultimately the most important parameter, as on the one hand very little overhead may result in a low robust transmission not allowing most users to recover the file, and on the other hand a very robust transmission consumes resources that could be used for other services.

\section{File Acquisition Probability Results}

Figure 5.7 shows the acquisition probability of a $16 \mathrm{Mb}$ file as a function of the number of bursts transmitted for different reception cases. We have assumed a constant download data rate of $250 \mathrm{~kb} / \mathrm{s}$ ( $1 \mathrm{Mb}$ burst size and $4 \mathrm{~s}$ cycle time between bursts). 

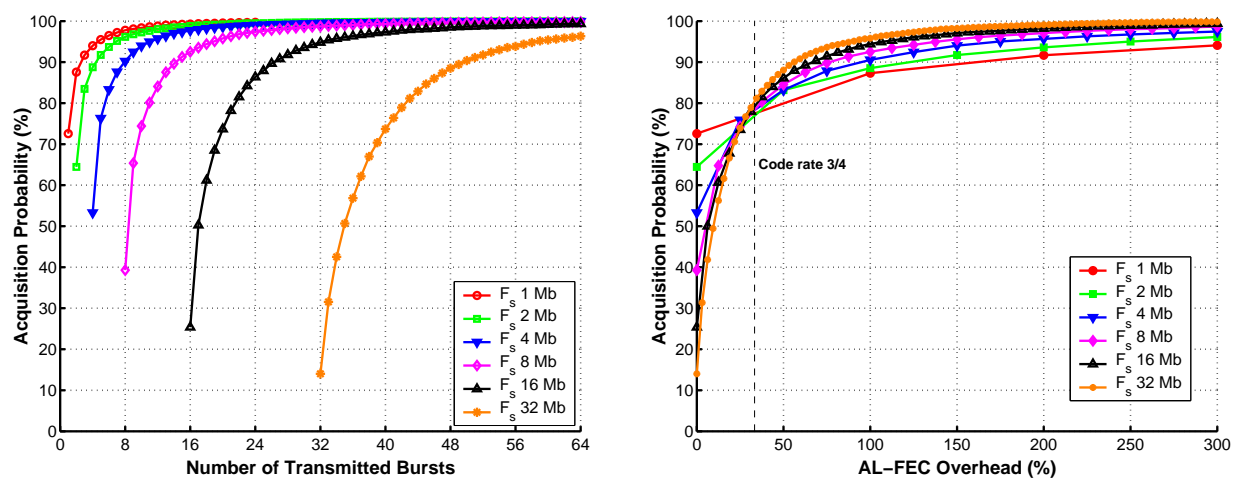

Figure 5.8: File acquisition probability versus number of bursts transmitted (left) and FEC overhead (right). Vehicular in-car reception.

The figure offers a guideline for system planning, in the sense of providing the right amount of parity data as a function of the reception case for a given targeted percentage of users receiving the file. Obviously, the more parity bursts transmitted, the higher the probability of receiving the file successfully. The gain is larger for higher receiver velocities (or larger bust cycle times). In the figure it can be observed that it is relatively easy to to serve vehicular in-car users with a network infrastructure dimensioned for outdoor pedestrian reception. In terms of link margin gain, that means that about $8.5 \mathrm{~dB}$ gain is achieved $(7 \mathrm{~dB}$ due to the additional vehicle penetration loss and $1.5 \mathrm{~dB}$ due to larger CNR requirement). However, the gain obtained by transmitting an additional parity burst decreases as the total number of parity bursts grows.

Figure 5.8 shows the acquisition probability of files of several sizes for vehicular incar reception as a function of the number of bursts transmitted and the AL-FEC overhead relative to the file size. File sizes of 1,2, 4, 8, 16 and $32 \mathrm{Mb}$ are considered. In the figures we can note that the transmission of larger files without any parity data becomes more difficult; as more bursts need to be received without any error. This case corresponds to the first marker to the left in each curve. On the other side, for moderate and large amounts of parity data transmitted large files require less FEC overhead than small files. This is because users experience more gain from the spatial diversity due to their mobility. Moreover, small files are also more vulnerable against complete burst loss. As a consequence, there is a code rate that provides an acquisition probability that is practically independent of the file size (around 3/4 in the example) [44].

\section{Post-Delivery Repair Data Results}

Figure 5.9(a) shows the cumulative distribution function (CDF) of the amount of repair data needed to decode a $16 \mathrm{Mb}$ file. The case without AL-FEC is also shown (which corresponds to 16 bursts containing only source IP data). 


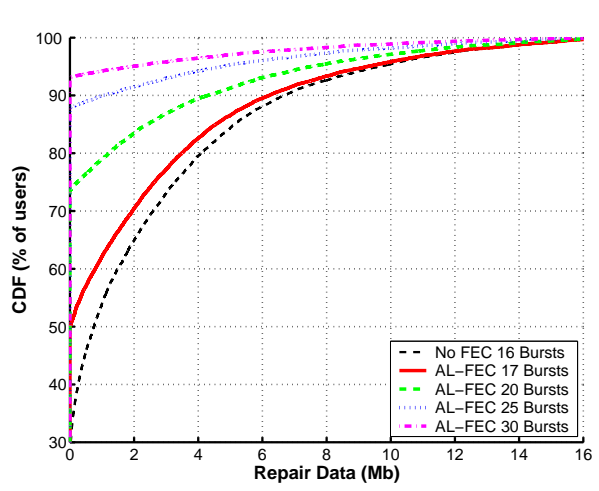

(a) CDF of the repair data needed.

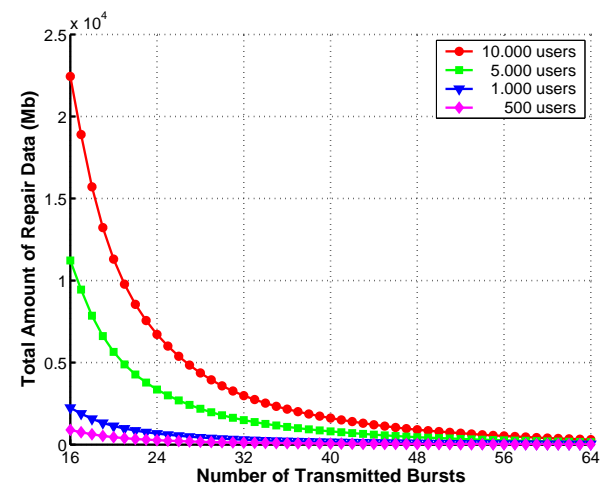

(b) Total amount of repair data.

Figure 5.9: Post-delivery results of a $16 \mathrm{Mb}$ file to vehicular in-car users as a function of the number of transmitted bursts.

The users that decode the file, they do not need any repair data, and thus in the y-axis it is plotted the acquisition probability for the different transmission configurations. In the figure we can note that in this example there are also some users that need a considerable amount of repair data. These users are ones located in bad DVB-H coverage areas that move at low speeds. Due to these reasons they will spend too much time in the outage areas. These users could be served more efficiently through the cellular network in a hybrid system is the cost of doing this is acceptable.

Now, let us now consider that a cellular network is used to deliver repair data on request. The same curves can be interpreted as a lower bound on the hybrid system performance under the assumption that bursts in DVB-H can be lost, but not the ones sent via the cellular network. The potential system efficiency improvement by using the cellular network for parity delivery can be seen studying the percentage of users that receive the file for each additional burst. For example looking at the curves for 25 and 30 bursts, about $5 \%$ of the users receive the file transmitting 5 more bursts (less than $1 \%$ for the 30 -th bursts). Sending the 30-th burst through the cellular network would save one DVB-H burst for another service. The cost efficiency of such approach will be determined by the actual number of users and the relative cost between broadcast and cellular resources.

Figure 5.9(b) shows the total amount to be repaired in the post-delivery repair session as a function of the number of bursts transmitted in the initial DVB-H file transmission and the number of active users per cell in the system (recall that there are 24 cells in the service area). Obviously, the amount of repair data that needs to be delivered with the cellular network increases linearly with the number of users, but we can see that it decreases exponentially with the number of transmitted bursts in DVB-H. 


\subsection{Filecasting in Hybrid DVB-H/3G+ Systems}

\subsubsection{File Delivery in Hybrid Cellular and DVB-H Systems}

A file delivery service in a hybrid cellular and DVB-H system with AL-FEC is, in essence, very similar to an MBMS file delivery service. In IPDC the use of MBMS is not yet standardized in the content delivery protocols [54]. The differences stem only from the change of the MBMS transmissions (both initial file transmission and optional repair transmissions) by DVB-H transmissions. The service consists in three phases as well: service advertisement phase, initial DVB-H file transmission phase, and error repair phase. Repair data bursts can be transmitted in the initial DVB-H transmission after the original source file has been sent; and to complete the reception of the file users served by default via p-t-p cellular connections. In case too many users fail to receive the file it is also possible to employ again DVB-H. The possibility of multicasting repair data through the cellular network with MBMS increases the flexibility of the system, especially in cells with poor DVB-H coverage and with moderate/high density of users. Similarly than in the previous section, it can be argued that the optimum delivery configuration in a hybrid DVB-H/3G+ system corresponds to a DVB-H/MBMS/HSDPA transmission. In case a DVB-H repair transmission is needed it would be more efficient to increase the duration of the initial transmission instead.

From an RRM point of view, the problem of efficient file delivery in hybrid cellular and broadcasting systems is more complex than when each network is operated separately. The reason is that an additional variable is needed to relate the importance of the resources of both radio accesses since they cannot be directly compared. As mentioned before, we adopt a cost-based RRM framework where the delivery cost is proportional to the amount of radio resources employed in each network. The broadcast cost is proportional to the amount of data transmitted in DVB-H and the cellular cost is proportional to the transmission energy (product of the transmit power times the transmission time). Assuming that the original source file is initially transmitted through DVB-H with a given burst size and cycle time, the optimum repair configuration will be the one that completes the delivery in due time and minimizes the delivery cost given by:

$$
C_{R}=c_{b} \cdot n_{b}+c_{c} \cdot \sum_{i} E_{c, i}
$$

where $n_{b}$ is the number of parity bursts transmitted in DVB-H, and $c_{b}$ and $c_{c}$ are the costs per transmitted DVB-H burst and per energy unit (J) of the cellular network respectively. One important figure is the number of cells within the service area, since it should be taken into account the total energy consumed by all cells. The optimum amount of parity data that should be transmitted in DVB-H will heavily depend on the ratio $c_{b} / c_{c}$. It should be noted that this value is difficult to quantify, because in practice it may vary over time in real life implementations. Prices may vary, for example, according to the congestion level experienced by each radio access (the cost of delivering certain amount of data over the cellular network during peak hours is higher compared to off-peak hours). 


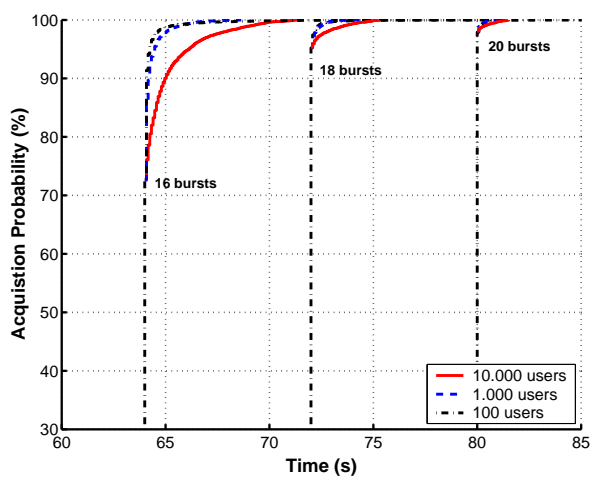

(a) Vehicular rooftop.

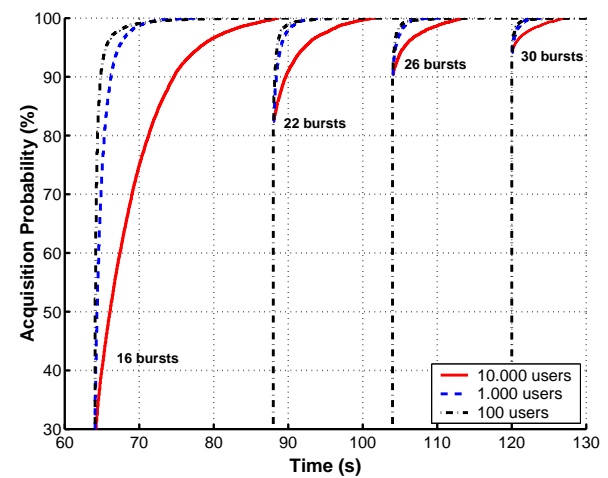

(b) Vehicular in-car.

Figure 5.10: File acquisition probability of a $16 \mathrm{Mb}$ file using DVB-H and HSDPA.

\subsubsection{File Delivery Results with DVB-H and HSDPA}

In this section we present simulation results for a file download service using DVB-H and HSDPA for vehicular rooftop and vehicular in-car DVB-H users ${ }^{2}$. In the simulations users not able to decode the file after the DVB-H transmission connect to the cellular network to complete the reception of the file. The cellular transmit power is again $2 \mathrm{~W}$. We investigate the optimum balance between the initial DVB-H transmission and the successive HSDPA error repair phase. Results focus on determining the operational cost savings that can be achieved using the cellular network as a function of the $c_{b} / c_{c}$ ratio and the number of active users.

Figure 5.10 shows the evolution of the acquisition probability of a $16 \mathrm{Mb}$ file as a function of the time for different number of transmitted bursts in DVB-H and for different number of active users. Recall that the first 16 bursts correspond to the original data file, and the rest are additional parity bursts with repair information. In the figure it can be seen that the cellular performance depends on the absolute number of users in the system. The larger the number of users, it will be more convenient to transmit more repair bursts in DVB-H. The optimum transmission configuration will depend on the relative cost of transmitting one additional repair burst in DVB-H compared to using the cellular network to serve a given percentage of users (ratio $c_{b} / c_{c}$ ).

Figure 5.11 shows the cost delivery savings that can be achieved combining DVB-H and HSDPA compared to the reference case where the DVB-H network and the HSDPA network are used separately. The cost saving is pictured as a function of the ratio $c_{b} / c_{c}$ for different numbers of active users in the system. In the situations that there are no savings the optimum configuration consists on using only DVB-H or HSDPA.

\footnotetext{
${ }^{2}$ It should be noted that the performance of the cellular network is the same for both reception cases, as the cellular network is limited by interferences. In contrast, the DVB-H network is limited by coverage.
} 


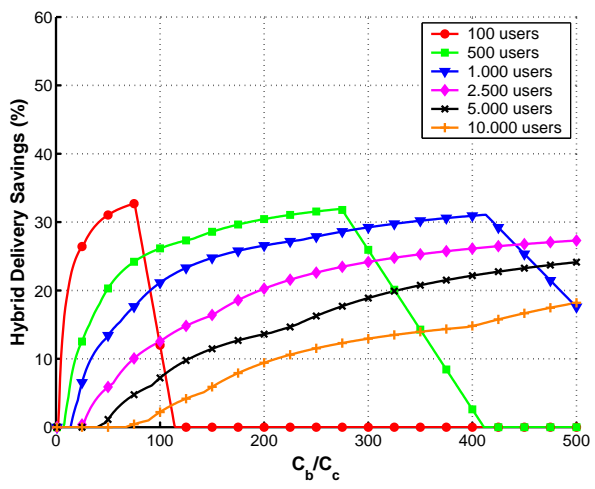

(a) Vehicular rooftop.

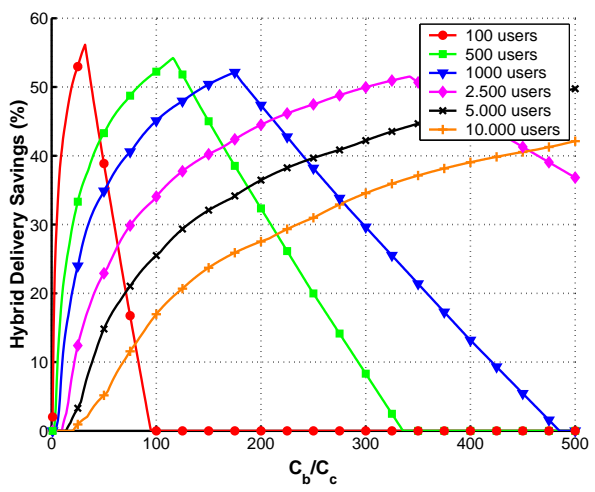

(b) Vehicular in-car.

Figure 5.11: Hybrid DVB-H/HSDPA delivery cost savings vs. Ratio $c_{b} / c_{c}$. Results are for $99 \%$ acquisition probability of a $16 \mathrm{Mb}$ file.

In the figure it can be noted that larger savings occur for the vehicular in-car reception case. The reason is that the number of parity data bursts required to achieve a target acquisition probability is considerably smaller. We can also observe that the savings initially increase as a function of the ratio $c_{b} / c_{c}$. In this area the broadcast cost is smaller than the reference case using only HSDPA. The maximum gain occurs for the $c_{b} / c_{c}$ ratio which provides the same cost using HSDPA and DVB-H separately. Larger $c_{b} / c_{c}$ values than this threshold imply that cellular network is more efficient than the DVB-H network. The maximum gain also depends on the number of active users in the system. The lower the number of users, the higher the maximum gain. However, the differences are rather small. For a given number of users there is a $c_{b} / c_{c}$ value which minimizes the delivery cost employing jointly DVB-H and HSDPA. In the figure savings of up to $35 \%$ for vehicular rooftop reception and up to $55 \%$ for vehicular in-car reception are achieved.

\subsubsection{File Delivery Results with DVB-H, MBMS and HSDPA}

If MBMS is considered, the efficiency of the cellular network is noticeably increased for the cases where the number of active users in the system is high. Figure 5.12 compares the cellular energy required to achieve an overall $99 \%$ acquisition probability of a $16 \mathrm{Mb}$ file as a function of the number of bursts transmitted in DVB-H for vehicular in-car reception using only HSDPA, and using both MBMS and HSDPA. We can see that MBMS only plays an important role for very large user densities and for low amounts of repair data transmitted in DVB-H.

In these cases the possibility of cellular multicasting with MBMS may reduce the repair cost of the DVB-H transmissions significantly compared to the reference case of using the broadcast network alone. However, the cost savings that can be achieved combining DVB-H, MBMS and HSDPA is reduced for large user densities compared to case with only DVB-H and HSDPA. 


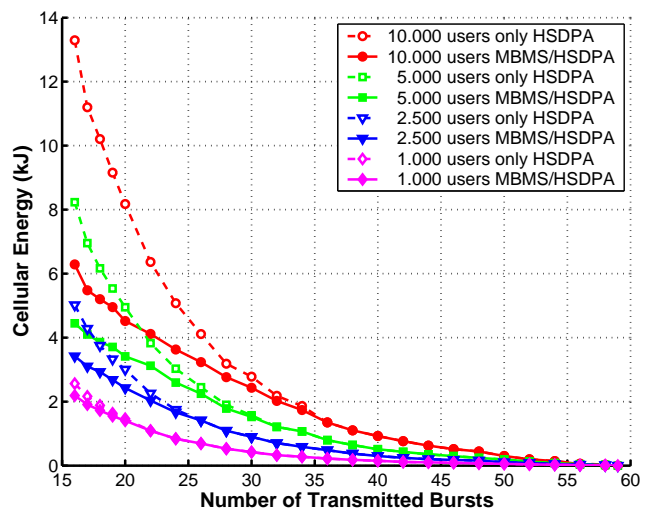

Figure 5.12: File delivery results using HSDPA and MBMS to repair the initial DVB-H file transmission. Cellular MBMS/HSDPA energy vs. number of transmitted DVB-H bursts. Results are for $99 \%$ acquisition probability of a $16 \mathrm{Mb}$ file for vehicular in-car users.

The reason is that the efficiency of the reference case using only the cellular network is also increased. This implies that the $c_{b} / c_{c}$ which yields the maximum gain for a given number of users occurs for smaller broadcast costs. The maximum gains shown in Figure 5.11(b) for 2.500, 5.000 and 10.000 users would be reduced down to 40\%, 35\%, and $30 \%$, respectively, if MBMS is considered.

\subsection{Conclusions}

In this chapter we have investigated radio resource management for multicast file delivery in hybrid DVB-H/3G+ systems using AL-FEC with Raptor coding. We have first evaluated the cases when HSDPA and MBMS are used independently in the cellular network. For HSDPA we have shown that the optimum transmit power is the minimum value that delivers the file in due time. A similar conclusion applies for MBMS, since if there are no time constraints to deliver the files it is more efficient to reduce the transmit power and increase the amount of Raptor parity data transmitted in order to benefit from the time diversity of the mobile channel along the transmission. As shown in Chapter 3 for DVB-H, the diversity gain with AL-FEC is higher for larger files and user speeds, enhancing the Raptor coding efficiency for the same proportion of parity data transmitted.

The problem with MBMS is that it can be very costly to serve the last percentage of users, especially if no macro-diversity technique is implemented. Hence, an important gain can be achieved employing HSDPA to serve the worst-case users. Obtained results show that from an overall system perspective it is not always efficient to serve nearly all users with MBMS, but rely on HSDPA to serve the final 20-30\% of users. Energy reductions up to $50 \%$ can be achieved. HSDPA outperforms MBMS in these cases because the transmissions are adjusted to the reception conditions of each particular user. Moreover, resources are only consumed when at least one user is actively using the service (with MBMS the transmission is active during the complete initial file transmission). 
The overall resource consumption reduction using MBMS and HSDPA jointly decreases in the situations when MBMS performs better. That is, the gain decreases for larger files, for lower file acquisition probabilities, and when implementing macro diversity techniques.

Regarding file delivery in DVB-H networks, we have shown how dynamic systemlevel simulations can be used to configure the transmission, in the sense of providing the right amount of parity data as a function of the file size for a given targeted percentage of users receiving the file. With AL-FEC file download services can be efficiently provided to mobile users over networks with partial coverage transmitting large amounts of repair data until the users receive the file. Our results show that it is relatively easy to to serve vehicular in-car users with a network infrastructure dimensioned for outdoor pedestrian reception. In terms of link margin gain, that means that about $8.5 \mathrm{~dB}$ gain is achieved $(7 \mathrm{~dB}$ due to the additional vehicle penetration loss and $1.5 \mathrm{~dB}$ due to larger CNR requirement). We have also shown that in these cases there is a code rate that provides an acquisition probability that is practically independent of the file size.

The file delivery efficiency in DVB-H networks that provide partial coverage of the service area can be significantly enhanced using a 3G HSDPA cellular network in areas with poor DVB-H coverage as a complement to deliver repair data. Cost delivery savings in the order of $50 \%$ are feasible in the situations where the content is popular enough that should be broadcasted with DVB-H. These savings may increase if the hybrid cellular and broadcasting system can take advantage of the opportunistic situations that may arise over time. The possibility of cellular multicasting with MBMS in the hybrid cellular and broadcasting system will significantly increase the flexibility of the system in areas with poor DVB-H coverage levels and moderate user densities. 


\section{Chapter 6}

\section{Conclusions and Future Work}

\subsection{Concluding Remarks}

This dissertation has treated cost efficient provisioning of mass mobile multimedia services with existing wireless infrastructure for digital broadcasting and cellular networks. Stateof-the-art mobile broadcasting (DVB-H) and cellular (evolved 3G+ networks with HSDPA and MBMS) commercial radio access technologies have been considered.

We have shown that DVB-H infrastructure deployment to support real-time delivery, high capacity (in the other of $10 \mathrm{Mb} / \mathrm{s}$ ), and nearly full area coverage as in traditional broadcasting is very costly. The conventional link layer protection mechanism MPE-FEC is only effective for streaming services ${ }^{1}$ in very dense networks with very good coverage level over the service area. Basically, it compensates for fast fading due to the mobility of the users in covered areas where static reception is possible. Instead, it is proposed to employ forward error correction with Raptor coding at the link (MPE-iFEC) or application layer (AL-FEC) to perform a multi-burst protection of the transmission. Contrarily to most technical enhancements proposed in the literature, the introduction of this technique would be backwards-compatible with existing networks and terminals, since it could be introduced as a software update.

Multi-burst FEC exploits the time diversity of the mobile channel to recover from temporary burst losses, which is not possible with MPE-FEC. It provides an enhanced protection against the impairments of the wireless channel. Multi-burst FEC can cope with temporary coverage discontinuities due to shadowing or path loss when passing through outage areas. Moreover, it also provides an improved protection against fast fading because it is possible to recover from heavily corrupted bursts. The transmission robustness with multi-burst FEC can be increased not only as a function of the capacity devoted for error repair, but also as a function of the number of bursts jointly encoded.

${ }^{1}$ With MPE-FEC the area coverage for file download services is smaller than for streaming services. An efficient delivery of large files that span several bursts is only possible in the areas where the burst error rate (with MPE-FEC) is close to zero. 
The gain obtained by performing a multi-burst protection of the transmission in an existing DVB-H network is twofold, as the area coverage is enlarged and the service quality perceived by the users in already covered areas with MPE-FEC is enhanced. Alternatively, multi-burst FEC can be used to reduce the required infrastructure cost to provide DVB-H services over a given area.

The drawback of multi-burst FEC is an increase of the network latency, since terminals must wait and store all bursts jointly encoded before decoding. The latency affects the user experience by delaying the initial reproduction of the services. Only services where a large latency is not an issue can fully exploit the benefits of multi-burst FEC without any drawback. Services that fall in this category are background file download services, and specific streaming services such as some video-clips, or news channel for vehicles in urban areas. In these cases it is possible to encode large amounts of bursts jointly and increase the time diversity of the mobile channel increasing the cycle time between bursts. However, for conventional mobile TV streaming services it is necessary to establish an adequate trade-off between the protection provided by multi-burst FEC and the latency introduced. Otherwise, the user experience would be compromised due a large zapping time. The actual latency values that will be tolerated by the users are today an open issue, but it is expected to be able to conceal latencies of around 10 seconds implementing so-called fast zapping techniques.

The problem with multi-burst FEC is that the service coverage is very difficult to assess in real life, because the probability of correctly receiving a burst depends on previous and/or future reception conditions. Therefore, the gain compared to MPE-FEC depends on the time evolution of the transmission errors experienced by the users, and hence on the velocity and trajectory of the users. The gain is not constant across the service area, and it cannot be directly included in the link budget. Only for relatively short latencies the gain can be expressed as a reduction of the fading margin required to cope with fast fading. In this dissertation we have developed a methodology to estimate the coverage for multi-burst FEC services in realistic scenarios based on dynamic system-level simulations. A computationally efficient performance model for the DVB-H physical layer based on 4-state Markov processes has been proposed and validated with laboratory and field measurements to yield good dynamic simulation performance with accurate mobility and radio signal level estimates.

Our performance evaluation results show that multi-burst FEC is especially suited for vehicular users because of their high speeds. Impressive link budget gains above $10 \mathrm{~dB}$ are feasible for services that tolerate large latencies, which would treble the area coverage. The potential gain is so high that to provide a target capacity it can be more efficient to increase the modulation order at the physical layer reducing the covered area, and increase the protection at the upper layers. However, it should be taken into account that the gain stems from the mobility of the users, and thus it does not hold for static users. In this sense, it is important the distribution of the outage areas across the service area, such that users do not become static for long periods in bad covered spots. 
For conventional mobile TV streaming services the gain is limited because of the zapping constraint. In this case the gain mainly stems due to the improved protection against fast fasting. Only vehicular users moving at very high speeds may cope with temporary shadowing. Nevertheless, interesting gains are also feasible employing very robust code rates. In particular, the area coverage can be doubled for vehicular reception, whereas for pedestrian reception the area coverage can be increased up to $50 \%$. For mobile TV services multi-burst FEC also yields an important gain in service quality, as it can practically erase all residual transmission errors in covered areas with MPE-FEC.

Multi-burst FEC will definitively enable a more affordable provisioning of mobile broadcasting DVB-H services ${ }^{2}$, but the interworking of DVB-H with a cellular network can further decrease the broadcast delivery cost. Especially if the DVB-H network provides partial coverage over the service area. In these cases there may be some users that experience significantly worse reception conditions than the majority (i.e., low mobility users in bad covered areas), such that they can be more efficiently served with the cellular network. In order to enable an easy and efficient interworking of the DVB-H and the cellular network $\mathrm{n}$ this case, multi-burst FEC should be implemented at the DVB-H application layer (AL-FEC). This cannot be achieved if multi-burst FEC is implemented in lower layers. With AL-FEC specific packets are not required to be retransmitted, and repair transmissions consist of additional repair packets that can be used for all users. Hence, repair data can be seamlessly delivered with p-t-p unicast transmissions and p-t-m broadcast/multicast transmissions. Our results considering an evolved $3 \mathrm{G}+$ cellular network with HSDPA for error repair of the DVB-H transmissions show that cost delivery savings in the order of $50 \%$ are feasible in the situations where the content is popular enough that should be broadcasted with DVB-H. Similar figures hold in evolved 3G+ cellular networks when HSDPA is used to repair errors of MBMS transmissions, but in this case the gain can be expressed in terms of cell capacity increase. The possibility of cellular multicasting with MBMS in the hybrid cellular and broadcasting system will significantly increase the flexibility of the system, especially in areas with poor DVB-H coverage levels and moderate user densities.

\subsection{Progressive DVB-H Network Deployment Discussion}

In this dissertation a framework for affordable provisioning of mass mobile multimedia services over hybrid cellular and broadcasting systems is proposed based on a progressive deployment of the DVB-H infrastructure. We argue that avoiding full DVB-H area coverage and very high capacity from the beginning is probably the key to providing affordable mobile broadcasting services.

\footnotetext{
${ }^{2}$ Raptor codes are patented by Digital Fountain, recently acquired by Qualcomm, and subject to intellectual property rights. Typical royalties for essential DVB patents are in the order of one euro per unit (for each compliant product).
} 
From a technical perspective, the combination of DVB-H with an evolved 3G+ cellular network into a hybrid cellular and broadcasting system presents a good potential to establish an efficient and flexible system for mass multimedia delivery to mobile users jointly utilizing the capabilities of existing broadcasting and cellular infrastructures. Moreover, it allows for an incremental deployment of the DVB-H infrastructure following the user demand, as the cellular network can be used in low populated areas until the deployment of a DVB-H transmitter or gap-filler is necessary.

On other other hand, in the initial phases of the DVB-H network roll-out it may not be necessary to provide such high capacity as the envisaged DVB-H commercial requirement of $10 \mathrm{Mb} / \mathrm{s}$. Recent studies using statistics obtained in mobile TV trials show that a relatively few number of broadcast bearers are nowadays required to complement the offering of cellular operators using 3G HSDPA [74], [73]. Although fixed TV services are defined by their program schedule, mobile users prefer to access content on-demand. Having the freedom to choose what to watch and when to watch it, being the only exceptions live events of high interest.

Reducing the broadcast capacity from $10 \mathrm{Mb} / \mathrm{s}$ down to $5 \mathrm{Mb} / \mathrm{s}$ (decreasing the modulation order at the physical layer from 16-QAM to QPSK with a code rate 1/2), would approximately double the area coverage per site. The coverage can be further increased with multi-burst FEC. Assuming about $300 \mathrm{~kb} / \mathrm{s}$ for mobile TV service, which corresponds to current commercial offerings, the number of TV channels that could be provided with multi-burst FEC code rates $1 / 4,1 / 3,1 / 2$ and $2 / 3$ would be $4,5,8$ and 10 respectively. Broadcasting initially only 4 or $5 \mathrm{TV}$ channels heavily protected with multi-burst FEC would drastically increase the area coverage of existing broadcasting infrastructure compared to the reference case with MPE-FEC and $10 \mathrm{Mb} / \mathrm{s}$. In the initial phases of the DVB-H network roll-out, additional sites should be only deployed where it is economically justified because the user density is large enough.

It should be pointed out that larger gains can be achieved for background file download services because it is possible to cope with both temporary shadowing and outage due to path loss. Moreover, the cellular network can be used to repair errors of the broadcast DVB-H transmissions reducing the broadcast delivery cost. In this sense, our results suggest that migrating from "linear" TV (real-time streaming) to solutions where content is transmitted during idle times and stored/cached in the terminals could lead to more costefficient ways of realizing mobile TV. These services are may open new revenue promising business opportunities, as the content can be delivered to the terminals beforehand and then played by the user, providing the illusion of instant transmission.

\subsection{Future Research Issues}

Several innovative research topics have been identified in this dissertation. A very hot research topic today is the integration of multi-burst FEC with fast zapping techniques for streaming services to reduce the zapping time perceived by the users. 
The recent adoption of a similar multi-burst FEC streaming framework than the one proposed in this work for DVB-H in the new DVB-SH standard known as MPE-iFEC has attracted a lot of attention to evaluate the actual latency values that will be tolerated by the users. Basically, fast zapping techniques play with the information arrangement (audio and video source data, and FEC parity data), and with adaptive media playout codecs able to slow down the multimedia stream playout [118]. An important aspect is to solve the transition between the fast zapping mode and the regular display mode (fully protected).

Future research is also needed to develop dynamic resource allocation algorithms for an efficient resource management in hybrid cellular and DVB-H systems, especially in the sense of optimally choosing the between different ways of delivering the data. From the service provider perspective, to make an optimal decision on the transmission configuration needs good knowledge about the reception conditions of the target terminals. The uplink channel of the cellular system can be used to transport quality measurements from the terminals to adjust the initial broadcast transmissions. However, tracking users' reception conditions may be impractical when the targeted number of users is large, and thus it may be useful to take the resource management decisions based on the statistical data collected from previous transmissions and field measurements/simulation experiments.

Another interesting research topic is to investigate the business opportunities of broadcast systems that deliver the multimedia content to the terminals beforehand, such that when it is played by the users they perceive an instant transmission ("push and store" services). In this direction, the impact on the broadcasting infrastructure design and dimensioning is also of interest. Note that this approach is valid for any wireless broadcast system, such as new mobile satellite services for example. Another business related topic is to investigate is how business relationships among the different parties involved affect the achievable performance of the hybrid cellular and DVB-H system for the several business models possible.

Finally, from this work several recommendations can be extracted regarding next generation of DVB mobile broadcasting technologies. The most important conclusion is that the focus should be on solutions to maximize the coverage, with the goal of providing wide area coverage at low cost. This is contrary to the usual requirement for future generation DVB systems, which focus on capacity increase. The main paths towards improved link budget are:

- Improved physical layer (air interface). During the standardization process of DVB-H it was decided to keep the DVB-T physical layer, which uses a concatenated ReedSolomon and convolutional code. This has been proven to be a mistake. Although it is possible to build shared DVB-T/H networks, dedicated DVB-H networks are eventually required to be able to dimension the network without any constraint imposed by DVB-T. Next generation DVB systems such as DVB-SH and DVB-T2 already provide enhanced air interfaces that approach closely the Shannon limit. DVB-SH employs turbo-codes, and DVB-T2 employs LDPC codes ${ }^{3}$. The problem is that these enhancements are not backwards-compatible with legacy DVB-H equipment.

\footnotetext{
${ }^{3}$ The gain compared to the DVB-H physical layer is about $3 \mathrm{~dB}$ reduction in the CNR requirement. A link budget improvement of $3 \mathrm{~dB}$ means about $50 \%$ increase of the area coverage.
} 
- Advanced antenna systems based on multiple antennas at the transmitter and receiver. Multiple input and multiple output (MIMO) antenna systems are currently being considered for most wireless communications systems. However, the UHF band, which can be considered optimum in the sense that it provides high bandwidth and maximizes the cell range, it is not practical to implement diversity techniques for handheld devices ${ }^{4}$. Only polarization diversity may be feasible. This topic is currently under study in DVB. Another solution may be to implement two antennas only at the transmitters. This is known as transmit diversity, and it has been adopted in DVB-T2 [150].

- Multi-burst forward error correction, as shown in this dissertation, can significantly enhance the transmission robustness for mobile reception taking advantage of the time diversity of the mobile channel. One alternative to improve its performance may be the proposed time-frequency slicing transmission pattern considered during the DVB-T2 standardization process [150]. This way, it may be possible to take advantage of both time and frequency diversity of the mobile channel, enhancing the coding efficiency to protect against temporary errors.

- Employ unequal error protection (scalable video coding) to different streams within the multimedia streaming service. The idea is to enhance the coverage with a more robust stream with lower resolution. This approach is considered for example in the Media FLO mobile broadcasting technology. One important consideration about this research topic is that it may be backwards-compatible with DVB-H, and thus it may further contribute to the provisioning of more affordable mobile TV DVB-H services [151]. The cross-layer FEC optimization at the physical, upper (link or application) and stream levels is a very challenging problem.

${ }^{4}$ This is feasible at the $\mathrm{S}$ band in DVB-SH because the antenna sizes are smaller. 


\section{Appendix A}

\section{DVB-H Performance Evaluation Methods}

Traditional network planning for both analogue and digital TV broadcasting networks is based on a static approach that targets to guarantee a certain coverage level (i.e., percentage of locations which average signal strength exceeds a given value with a target high probability). However, mobile broadcasting networks require dynamic analysis over time as well as in cellular networks, since the actual service quality perceived by the users depends on the time evolution of the transmission errors experienced by the users [102]. Therefore, quality of service issues in DVB-H cannot be studied only from performance indicators that reflect an average within the service area, such as the coverage probability or the average burst error rate commonly used in network planning [117].

\section{A.1 Field Measurements}

Field measurements are the most accurate way to measure the actual performance of any wireless communication system. Ideally, transmitter and receiver equipment and necessary resources are available, and performance analyses can be accomplished in field conditions. Additionally, field measurements can be complemented with laboratory measurements using a channel emulator. During this dissertation field measurements were performed in the DVB-H test-beds of the universities of Turku (Finland) and Braunschweig (Germany), see Figure A.1 .

\section{DVB-H Pilot of the University of Turku}

The test-bed is a single frequency network with two transmitters covering the city center operating at $610 \mathrm{MHz}$ (channel 38). It is dimensioned for pedestrian outdoor reception. Figure A.1 shows a map of Turku with the location and characteristics of the two transmitters. 

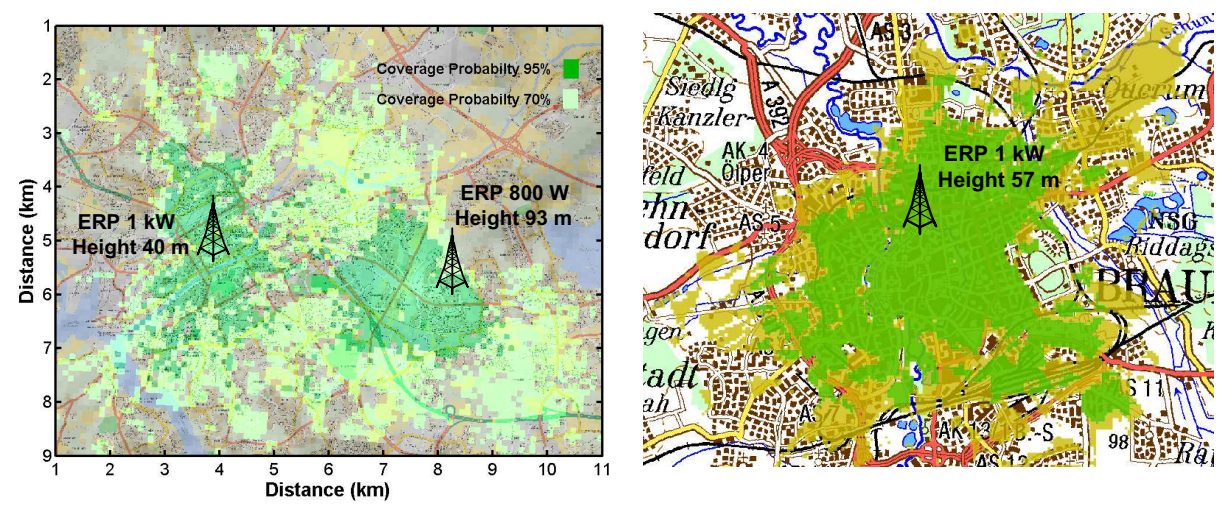

Figure A.1: Characteristics of the DVB-H test networks of Turku (left) and Braunschweig (right).

Figure A.1 also depicts the area coverage for pedestrian outdoor reception for the DVB-H physical layer transmission mode employed in the measurement campaign (FFT size $8 \mathrm{~K}$, OFDM symbol guard interval 1/4, modulation 16-QAM, and code rate 1/2), and MPE-FEC 3/4 at the link layer.

\section{DVB-H Pilot of the University of Braunschweig}

The test-bed consists of one transmitter with three sector antennas operating at $554 \mathrm{MHz}$ (channel 31). The antennas are mounted at a height of $57 \mathrm{~m}$ in azimuths of $45^{\circ}, 225^{\circ}$ and $315^{\circ}$, and have a beam width of $65^{\circ}$. The $45^{\circ}$ antenna has a down-tilt of $10^{\circ}$, the others $0^{\circ}$. The total transmit power is $1 \mathrm{~kW}$ ERP (effective radiated power), which is split equally among the antennas (55 dBm each). Figure A.1 shows a map of Braunschweig with the location of the transmitter, and the area coverage for vehicular outdoor reception for the physical layer transmission mode employed during the measurements (FFT 8K GI 1/4 16-QAM 2/3), and MPE-FEC 3/4 at the link layer.

\section{Measurement Set-Up}

The measurement set-up employed in Turku and Braunschweig consisted of two receivers with a common external antenna and a GPS receiver. The GPS was used to record terminal position and speed. The receivers employed in Turku were two Nokia DVB-H prototype receivers employed in the European Wing TV project [138]. One receiver was used to record RSSI (Received Signal Strength Indicator) with $1 \mathrm{~dB}$ resolution and the other to record the TS packet error trace. The receivers employed in Braunschweig were two commercial DVB-T USB receivers from Pinnacle. Both receivers were used to record TS packet error information, but one receiver was additionally attenuated to enable a comparison of two signals with a constant offset in the received power. The measurement systems are illustrated in the block diagram of Figure A.2. 

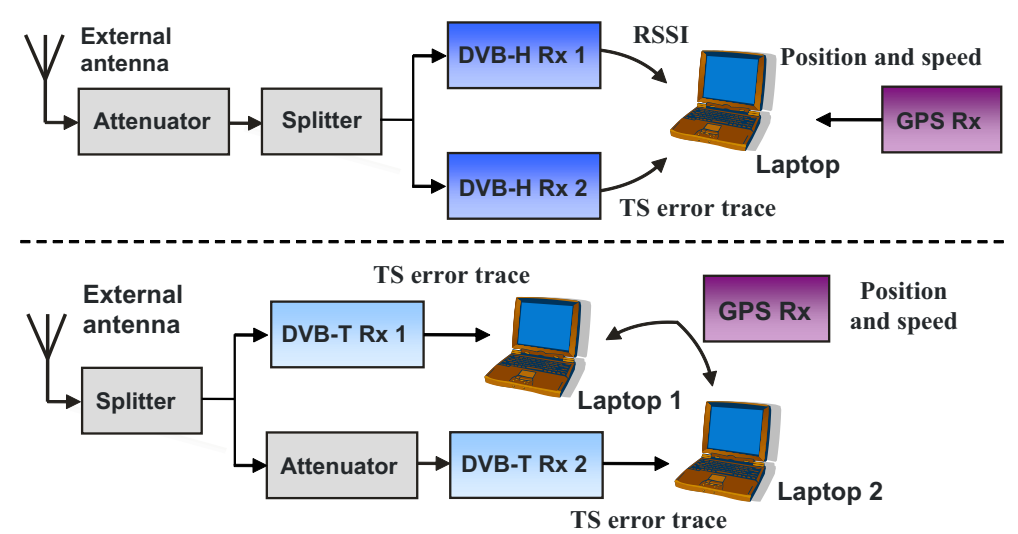

Figure A.2: Field measurement systems employed in Turku (above) and Braunschweig (below).

Measurements were performed for vehicular urban and sub-urban reception conditions at various locations and transitions around the cities of Turku and Braunschweig, with maximum speeds of approximately $60 \mathrm{~km} / \mathrm{h}$. We considered traces with a wide range of transmission errors to investigate the maximum error correction capability of upper layer FEC. Additionally, some vehicular motorway (with approximately constant speeds of 100 $\mathrm{km} / \mathrm{h}$ ), and pedestrian indoor and outdoor measurements performed in Turku during the Wing TV project [52] were analyzed to study the influence of different reception conditions. Figure A.3(a) shows an example of the data recorded during the DVB-H field measurement campaign in Turku.

Basically, the measurements consist of MPEG-2 TS packet error information at the physical layer of the whole multiplex. This can be done in real-time by storing the TS packet header field called TEI (Transport Error Indicator), that indicates whether the packet has been correctly received or not. Another possibility is to transmit a packet counter in each TS packet. By recording the packet error trace at the physical layer it is possible to reproduce the quality experienced by the measuring terminals across the measured trajectories for any type of service emulating the upper layers in software (time-slicing, FEC mechanisms and protocol decapsulation). Note that the measured error traces depend on the physical layer transmission mode and network configuration employed, and thus these parameters are fixed. Also, as we are recording information only at the physical layer, it is possible to employ DVB-T receivers to study compatible physical layer configurations [139].

As an example of the results that can be obtained from a field measurement, Figure A.3(b) shows the cumulative number of erroneous seconds in the measured trajectory for a six-minute streaming service at $384 \mathrm{~kb} / \mathrm{s}$ for different MPE-FEC code rates. To obtain the results we have considered the conventional MPE-FEC erasure decoding mode and 512 rows per MPE-FEC frame (burst) a maximum burst size of $1 \mathrm{Mb}$. We have also assumed a constant IP packet size equal to 512 bytes, and that each IP packet can be played successfully without the need of any previous IP packet. In the figure we can see the time evolution of the errors and the actual MPE-FEC performance perceived by the users. 

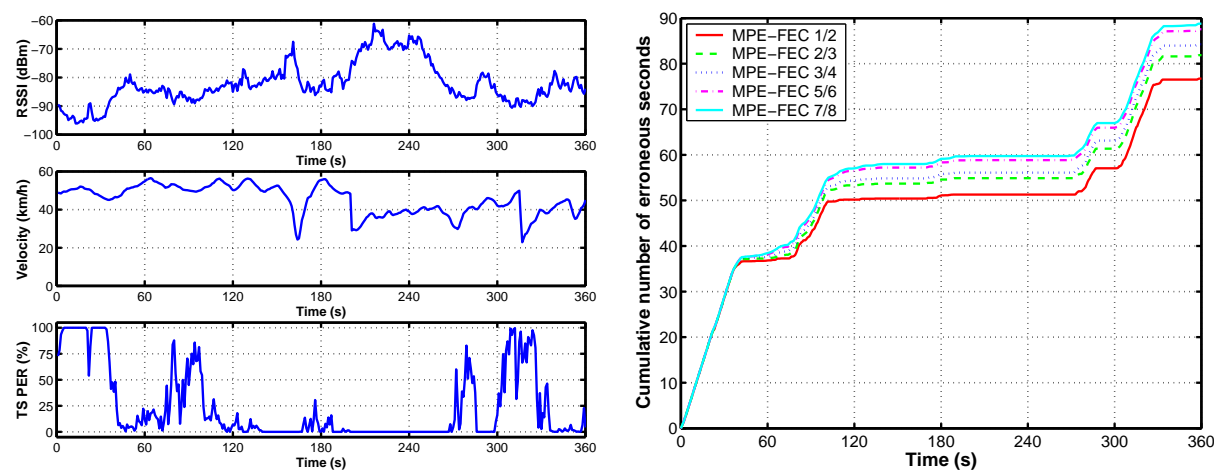

(a) RSSI, velocity, and TS PER.

(b) Time evolution of the erroneous seconds for a $384 \mathrm{~kb} / \mathrm{s}$ streaming service.

Figure A.3: Example data of vehicular DVB-H field measurement.

\section{A.2 Dynamic Simulations}

The main issue with field measurement campaigns is that their resource expenditure is often cost prohibitive. Moreover, results obtained apply only for the specific trajectories measured. In order to extract conclusions about the overall system performance experienced by users in a service area, a considerably large number of measurements are needed. If, for example, we would like to speak in terms of percentage of satisfied users for a given quality of service (QoS) satisfaction criteria, thousands of independent trajectories would generally be needed to obtain statistically consistent average results. If we take into account that the measurements are only useful for the specific DVB-H physical layer transmission mode and network configuration setting employed, the need of performing simulations becomes apparent.

Simulations are an essential tool when analyzing the performance of wireless communication systems. With simulations different transmission configurations can be compared and the system parameters can be tuned to maximize performance. Obviously, simulations do not replace field measurements, and results should always be verified with field measurements in the scenario under study. But still the potential cost reduction is considerable.

Looking carefully at the field measurement depicted in Figure A.3(a), we can distinguish between three different processes that need to be modeled in order to obtain similar results. First of all, a mobility model is necessary to determine the trajectories and velocities of the users. Secondly, it is needed to model the wireless radio channel to predict the signal strength received by the mobile terminals. Finally, a DVB-H physical layer model is also required to determine which information is correctly received based on the time-variant reception conditions as indicated by the received signal strength and receiver velocity. It should be pointed out that we are interested in accurate and computationally efficient performance models, but not in modeling the complete physical layer with a physical layer simulator. 

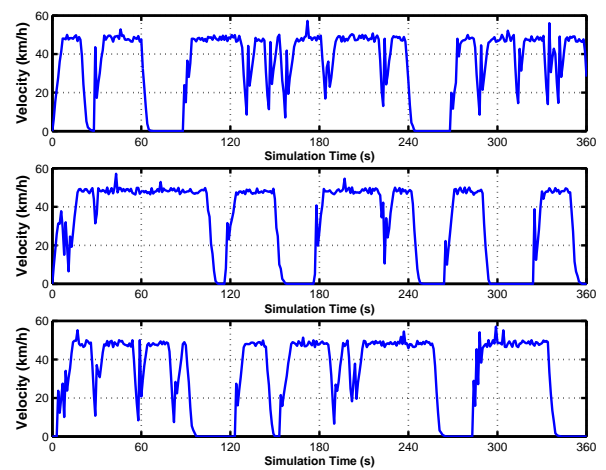

(a) Velocity prediction using SUMO.
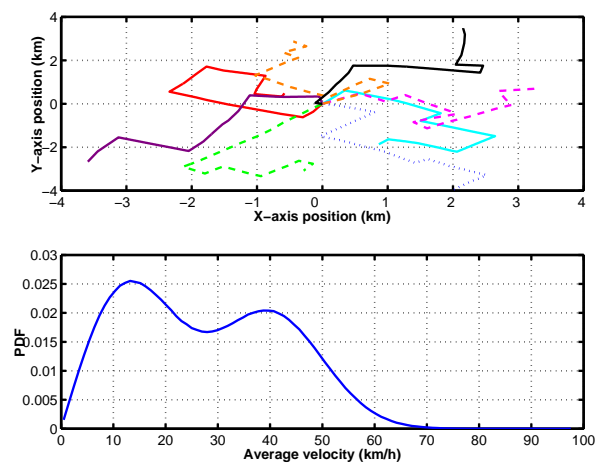

(b) Street pattern and velocity probability density function using the statistical model.

Figure A.4: Example of results provided by the mobility models considered in the dissertation.

The simultaneous simulation of all the processes involved in the operation of a wireless communication system is very complex and time consuming. The commonly employed solution is to separately characterize and model the receiver performance at the physical or link layer. At system level, where many transmitters and receivers may be considered, individual receivers are not simulated but the performance mode.

\section{A.2.1 Mobility Models}

Mobility models describe the movement pattern of mobile users, and how their location, velocity and acceleration change over time. Since mobility patterns may play a significant role when evaluating the system performance, it is in general desirable that the model is based on real traffic patterns of the scenario under study, or at least that the movement is emulated in a reasonable way. Otherwise, the observations made and the conclusions drawn from the simulation studies may be misleading. Nevertheless, very detailed models are also very complex and require information that is not always available (e.g., street vector information), and thus there is a trade-off between model accuracy and complexity.

A good example of a very sophisticated vehicular model is the simulation environment SUMO (Simulation for Urban MObility) [133], which employs streets vector information and takes into account traffic lights, turning probabilities, car densities etc. This model was used to perform dynamic simulations in the scenario of Braunschweig. Figure A.4(a) shows different realization examples of the simulator. Note how the time evolution of the velocity predictions resembles quite well the velocity measured in the field measurement depicted in Figure A.3(a).

A good example of a statistical model for vehicular users relatively simple to implement but still able to provide realistic street patterns and terminal movements is [135]. The model captures the users' movements with three random variables: street distance, direction change at crossroads and average velocity to find an analytical formulation. 
Table A.1: Statistical vehicular model parameters.

\begin{tabular}{|c|c|c|c|c|c|c|c|c|}
\hline$p_{0^{\circ}}$ & $p_{90^{\circ}}$ & $p_{-90^{\circ}}$ & $p_{180^{\circ}}$ & $\bar{d}$ & $\bar{v}$ & $\bar{v}_{m r}$ & $\sigma_{v}$ & $p_{m r}$ \\
\hline \hline 0.595 & 0.25 & 0.15 & 0.005 & $250 \mathrm{~m}$ & $10 \mathrm{~km} / \mathrm{h}$ & $40 \mathrm{~km} / \mathrm{h}$ & $10 \mathrm{~km} / \mathrm{h}$ & 0.5 \\
\hline
\end{tabular}

Basically, the model assigns to the users a new velocity, street distance, and a relative change in direction when they finish moving across their current street. This model was used to perform dynamic simulations in the scenario of Turku. The model provides different distribution functions with a limited number of parameters that can be easily derived for a particular city. The relative direction changes at crossroads are expressed by four random variables associated to turning left $p_{-90^{\circ}}$, right $p_{90^{\circ}}$, round $p_{180^{\circ}}$, and continuing straight ahead $p_{0^{\circ}}$. They are assumed to be normally distributed with the same standard deviation $\sigma_{\varphi}$, equal to $\pi / 32$. The street distance random variable is assumed to be Rayleigh distributed, with an average length $\bar{d}$. Finally, the users' speed is modeled according to a Rayleigh/Rice distribution to distinguish between urban streets and major roads. The velocity parameters are the mean velocities of city and major roads, $\bar{v}$ and $\bar{v}_{m r}$, velocity deviation $\sigma_{v}$, and percentage of cars on major roads $p_{m r}$. Table A.1 shows the parameters used in the simulations. Figure A.4b shows different street pattern realizations and the probability density function (PDF) of the average velocity for the parameters considered.

\section{A.2.2 Modeling the Wireless Radio Channel in Single Frequency Networks}

The wireless channel between a transmitter and a receiver is traditionally modeled with three different processes: path loss, slow or long-term fading (shadowing), and fast or multipath fading, see Figure A.6 [121]. All of these processes vary as the positions of the transmitter and receiver change, and as any contributing object between the antennas is moved. SFNs differ from conventional wireless communication systems in the sense that all transmitters are frequency and time synchronized, which allows receivers to combine OFDM signals coming from several sites. Therefore, an OFDM signal combining model is required in order to predict how signals from the different sites contribute to the useful received signal or cause self-interference at each location [140].

\section{Path Loss}

The path loss is the overall decrease in field strength as the distance between the transmitter and the receiver increases. The physical processes behind it are the outward spreading of the radio waves from the transmit antenna and the obstructing effects of trees and buildings. For large distances, the received signal strength decreases exponentially with the distance, and the path loss can be in general modeled as:

$$
L_{d}=\beta+10 \cdot \alpha \cdot \log _{10}[d],
$$

where $\beta$ is a distance independent term and $\alpha$ is the propagation exponent. For free-space propagation $\alpha$ is equal to 2 , but in practice it usually ranges between 3 and 4 . 


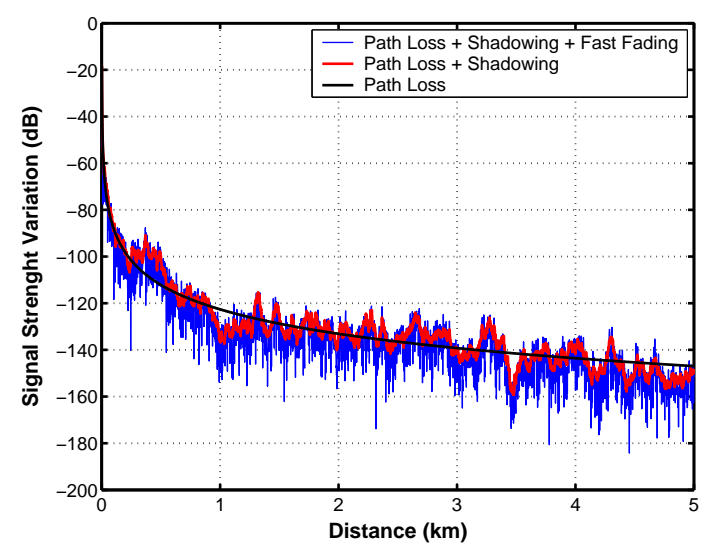

Figure A.5: Wireless channel as a superposition of path loss, shadowing and fast fading.

In order to calculate the attenuation as precisely as possible in real scenarios, numerous radio propagation models have been developed, although the accuracy of the results will always depend on the available geographic data and its resolution. Geographic data can be classified in terrain height (digital terrain model), terrain morphology (land usage or clutter class), and building heights and shapes. In order to further increase the accuracy of the results, radio propagation models can be calibrated based on field measurement campaigns. The calibration process aims to provide a zero mean and a minimum standard deviation of the error between the prediction and the measurements. After calibration a good prediction model is expected to show a stable mean value of approximately $0 \mathrm{~dB}$ and a standard deviation at the scale of 6 to $8 \mathrm{~dB}$.

Deterministic ray-based propagation models can provide accurate path loss predictions in complex propagation environments such as dense urban areas. However, practical limitations including computation time and the need of very detailed cartography, which is expensive and difficult to obtain, prevent these models of being used for large-scale radio network planning. For these reasons in practical applications empirical and semi-empirical models are commonly used. Empirical models are based on extensive field measurement campaigns, whereas semi-empirical models are based on a mix of empirical and theoretical data. The main advantage is that they can be expressed as closed equations dependent on several parameters that can be easily derived. However, it is especially important to calibrate these models with field measurements to adjust the model to the particular scenario under study.

The coverage map for the scenario of Turku was computed by the Finnish broadcasting service provider Digita within the Wing TV project, using the Canadian research corporation propagation model [141], terrain height, building and clutter information (pixel size is $110 \mathrm{~m}$ ). For the scenario of Braunschweig, the macro cell propagation model proposed in [134] was employed, using ground height and clutter data with a $50 \mathrm{~m}$ resolution. 

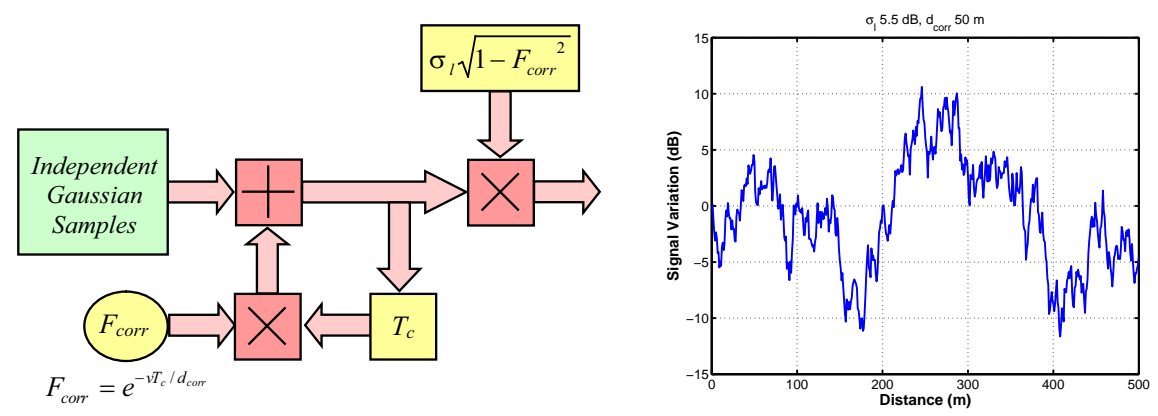

Figure A.6: Method for simulating correlated shadowing. Simulation parameters: shadowing standard deviation $\sigma_{l}$, shadowing correlation distance $d_{c o r r}$, and user velocity $v . T_{c}$ is the simulation sampling time.

\section{Shadowing}

Shadowing models the deviations from the mean distance-dependent signal level due to the particular obstructions (clutters) between the transmitter and the receiver. It changes more rapidly than the path loss, with significant variations over distances of several tens of meters (comparable to the widths of buildings in the region of the mobile). Shadowing is traditionally assumed to be log-normally distributed. That is, the distribution of the signal power is log-normal (the signal measured in $\mathrm{dB}$ follows a normal or Gaussian distribution). It is also sometimes referred to as log-normal fading.

The spatial variation of the shadowing in $\mathrm{dB}$ is described by a zero-mean Gaussian random variable with standard deviation $\sigma_{l}$ and correlation distance $d_{c o r r}$. The standard deviation of the shadowing distribution is known as the location variability, and it determines the spread of the signal field strength around the mean value. Its value increases with frequency, being greatest in suburban areas and smallest in open areas. It is usually in the range of 5 to $12 \mathrm{~dB}$. The value usually employed for outdoor broadcasting signals in the UHF band is $5.5 \mathrm{~dB}$ [34], although it should be pointed out that it depends on the resolution of the path loss prediction, and that for high resolution maps (e.g., $5 \times 5 \mathrm{~m}$ ) a lower value may be more realistic. Spatial correlation of shadowing is usually modeled using a first-order exponential model [120]:

$$
\rho(d)=e^{-d / d_{\text {corr }}},
$$

where $d_{\text {corr }}$ is distance taken for the autocorrelation to fall by $e^{-1}$. To simulate correlated shadowing we employ the methodology proposed in [121], depicted in Figure A.6.

\section{Fast Fading}

Fast fading accounts for the signal level fluctuations due to constructive and destructive superposition of the multiple signals reaching the mobile terminal. It involves variations on the scale of a half-wavelength, and frequently introduces variations as large as 35 to 40 
$\mathrm{dB}$. The variation of the attenuation in $\mathrm{dB}$ is typically characterized by a Rice distribution under line of sight (LoS) conditions, and by a Rayleigh distribution under non-line of sight (NLoS) conditions.

In DVB-H, the time variant small-scale fluctuations of the received signal due to fast fading are modeled together with the frequency shifts of the received signal due to Doppler effect within the so-called channel models. Note that channel models are used not only in simulations but also in laboratory measurements with the channel emulators. The maximum Doppler can be computed as:

$$
f_{d}=v \cdot \frac{f_{r f}}{c}
$$

where $v$ is the receiver speed, $f_{r f}$ is the carrier frequency of the transmission, and $c$ is the speed of light. For the UHF band where DVB-H was originally designed to be deployed (470-862 MHz), Doppler frequencies above $10 \mathrm{~Hz}$ are associated to vehicular reception, whereas Doppler frequencies around $1-3 \mathrm{~Hz}$ are associated to pedestrian reception (e.g., 2 $\mathrm{Hz}$ correspond to $3.6 \mathrm{~km} / \mathrm{h}$ at $600 \mathrm{MHz}$ ).

The typical urban 6-tap (TU6) channel model developed within the COST207 action [142] has been shown to be representative for DVB-H vehicular reception conditions [14]. This channel model reproduces a typical terrestrial profile in an urban area and consists of 6 discrete paths. For slowly moving pedestrian reception, specific tapped delay line channel models for DVB-H have been proposed, which are known as PI/PO (Pedestrian Indoor/Pedestrian Outdoor) channels, as it was observed that the TU6 model provides pessimistic results [122], [143]. The channel models have been developed by the Wing TV project based on field measurements in SFNs. The TU6 and PI/PO profile parameters can be found in Annex D of [117].

In addition to the Doppler frequency, for specifying the TU6 and PI/PO models it is necessary to determine the average carrier-to-noise ratio (CNR) of the channel, which describes the ratio of the useful signal power to the sum of the interference and noise powers. In DVB-H noise and interferences are additive, since signals consist of thousands of carriers modulated in phase and amplitude, resembling a Gaussian noise signal, and having a flat noise-like spectrum where its energy is distributed over the whole RF channel.

\section{OFDM Signal Combining Models}

In SFNs interference consists of both external interferences and self-interference from the own network. For small-size networks, self-interferences are negligible, but they cannot be neglected in a dense SFN [130]. Signals received within the OFDM symbol guard interval are considered as useful and contribute totally to the useful signal, whereas signals with a time delay larger than the guard interval cause self-interference. In practice, signals arriving with a slightly longer delay than the guard interval contribute partially to the useful signal and partially to the self-interference. Usually, a weighting function according to the signal delay is employed to determine the ratio between the useful and interfering contribution [140]. 
The performance of the receivers will strongly depend on the synchronization strategy used to determine the time synchronization point (FFT window time position). A good overview of the different synchronization strategies can be also found in [140]. The optimum FFT window time position where the effective CNR is maximized cannot be easily found, and in general would take too much time to be calculated. Therefore, normally a simpler strategy is applied, such as synchronizing to the strongest signal, or synchronizing to the first received signal that exceeds a given threshold.

When computing the CNR, it should be taken into account that both useful and interfering signals are assumed to have log-normal power distributions, which may be correlated [144]. A good overview of different summation methods of log-normal components can be found in [145].

\section{A.2.3 DVB-H Coverage Prediction}

The traditional approach for the planning of broadcasting networks consists in defining the required minimum median field strength that must be offered in a target area, and then predict the locations where this level can be reached. Using a radio propagation model and an OFDM signal combining model with system information about transmitters and receivers characteristics (link budget), only the mean signal strength can be predicted with a spatial resolution given by the available cartography. Typical link budget values for DVB-H network planning can be found in [134].

For coverage prediction, signal fading effects due to shadowing and fast fading cannot be ignored. They make coverage prediction statistical, such that it is predicted the signal availability rather than the signal level. Fast fading it is taken into account in the minimum CNR requirement. Usually the threshold is set to achieve an average burst error rate below $5 \%$. On the other hand, to account for the local shadowing effects within each grid element of the coverage map a fading margin of several decibels is added to the link budget. This margin (also known as correction location factor in broadcasting) can be computed as:

$$
C_{l}=\mu \cdot \sigma_{l},
$$

where $\sigma_{l}$ is the shadowing standard deviation and $\mu$ is the distribution factor that depends on the coverage probability target. It is equal to 0.52 for $70 \%, 1.28$ for $90 \%, 1.64$ for $95 \%$, and 2.33 for $99 \%$. In DVB-H network planning the quality of coverage in a small area (coverage map grid resolution) is classified as "acceptable" and "good" for pedestrian reception if at least $70 \%$ and $95 \%$ of the receiving locations within the area are covered respectively. For vehicular reception the threshold coverage probabilities are increased to $90 \%$ and $99 \%$ of receiving locations for good and acceptable coverage respectively [134].

\section{A.2.4 Modeling the DVB-H Performance}

The DVB-H standard works with MPEG-2 TS packets at the physical layer (size 188 bytes), and IP packets at the link layer (typical size 0.5-1.5 kB). Each time-sliced burst consists of a number of MPE (Multi Protocol Encapsulation) sections, and each IP packet is encapsulated into one section. 
At the receiver, the physical layer FEC corrects bit errors within TS packets, and each packet is considered either completely received or completely erroneous. MPE sections carry a cyclic redundancy check field, and thus it is also possible to consider them either correct or lost. A DVB-H performance model shall then predict which TS packets or MPE sections are correctly received per burst. We can distinguish between physical layer(TS level) and link layer (MPE section level) performance models. The link layer model has the advantage of a reduced complexity, as the number of MPE sections per burst is smaller than the number of TS packets per bust, which will speed up the simulations. However, this requires the IP packet size to be constant, which is not generally true. Also, performing simulations at the TS packet level provides more possibilities for post-processing and reuse of the obtained error traces. Some applications require working at the TS packet level, as an erroneous section can contain several correct TS packets. Note that in the worst case one single erroneous TS packet can cause the loss of up to two MPE sections. In practice TS packet error patterns are heavily correlated such that several consecutive packets are lost, and the loss rate at the link layer is almost the same than at the physical layer. The input parameters for a DVB-H physical layer model should be the following:

- Physical layer transmission mode.

- Channel model.

- Carrier-to-Noise Ratio.

- Doppler frequency.

The transmission mode is fixed for any given simulation scenario. It is mainly determined by the desired system capacity, although other parameters such as transmission robustness, maximum supported terminal velocity, and maximum distance between transmitters are also taken into account in the design process. The channel model may be considered fixed as well, e.g., TU6 for vehicular reception and PI/PO for pedestrian reception. Therefore, the only time-variant parameters are the CNR and Doppler frequency, which should be updated in short time intervals such that the receiving conditions can be considered piecewise stationary. In our case that means that path loss and shadowing can be considered constant, such that the only variations are due to fast fading. A good practice is to work with a time resolution equal to the burst duration, with typical values around 0.2-0.4 seconds.

\section{A.2.5 System-Level Simulations}

With the models described in the previous sections it is possible to simulate a DVB-H network over time, being able to estimate the QoS experienced by the users in a very detailed manner, giving a better understanding of the network behavior. These simulations are usually referred to as system-level simulations, and are widely used in cellular networks. Dynamic system-level simulators allow evaluating the overall system performance perceived by the users statistically with Monte Carlo simulations. This means repeating the same experiment many times with different random seeds and computing the average results (the higher the number of repetitions, the more accurate the results). 


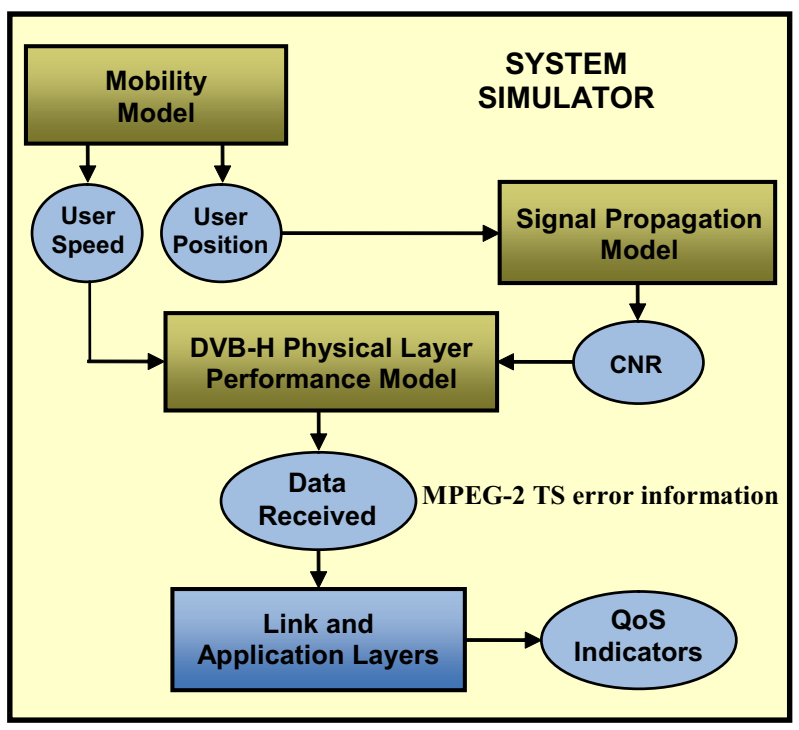

Figure A.7: DVB-H system-level simulator architecture.

In the particular case of DVB-H, such system-level simulations can be used as a complement of traditional coverage planning tools for broadcasting networks for analyzing QoS and radio resource management (RRM) aspects of the transmission configuration. For example, it would be possible to monitor the time evolution of the errors of a streaming service perceived by the users, or determine the users that successfully receive a file (and the amount of repair information needed by each user not able to decode the file). Another application would be to evaluate the influence of additional sites not only in terms of area coverage, but also in terms of QoS perceived by the users.

Figure A.7 shows the architecture of the DVB-H system-level simulator developed in this dissertation [94]. The simulator can be considered a discrete-event simulator, where the only events are the reception of data bursts by the users. Network dynamics are represented as a chronological sequence of events, simulated with a slot resolution equal to the cycle time between data bursts (time at which mobiles' movement and received signal strength are updated and data received in the burst is computed). Application examples of the simulator can be found in chapters 3,4 and 5 .

\section{Mobility Module}

This module moves users across the service area and computes the speed of the users when receiving a burst. Obviously different models are needed to account for the different user cases: pedestrian (indoor and outdoor) and vehicular (urban and motorway). Another important aspect is how to distribute the users initially over the service area. Typically they are distributed uniformly, unless specific information about population density is available. 


\section{DVB-H Radio Coverage Module}

This module computes the average CNR during the reception of a burst for each user taking into account path loss and shadowing (and OFDM signal combining effects in SFNs). Recall that fast fading is accounted in the DVB-H performance module. This module can be associated with a traditional radio propagation tool employed for digital TV network planning. A coverage map of the service area can be pre-computed in order to speed up the simulation process. The coverage is evaluated in a grid of test points over the service area, and the values in-between are obtained by means of interpolation. Usually coverage predictions do not include shadowing (only the mean path loss), and thus it has to be simulated for each user using the method described in the previous section.

\section{DVB-H Performance Module}

This module computes which TS packets of a burst are correctly received for each user, based on the CNR and vehicle speed information provided by the radio coverage and mobility modules.

\section{DVB-H Upper Layers Module}

This module emulates the protocol decapsulation and FEC decoding at the link and application layers, and computes the QoS metrics. Common metrics for streaming services in DVB-H are: burst error rate, also known as MFER (MPE-FEC Frame Error Rate), IP PER (IP Packet Error Rate), ESR (Erroneous Second Ratio), and ESR5(20), which represents the percentage of time intervals of 20 seconds with at most 1 erroneous second. For file delivery services the main indicator in terms of QoS is whether the user receives the file correctly or not. Additionally, the time required to receive the file is also of interest.

\section{Small Time-Scale Simulations}

Finally, we want to point out that depending on the time scale of the simulations and the mobility of the users it may not be necessary to consider all three wireless channel processes: path loss, shadowing and fast fading. Path loss variations only play an important role on large time scales (e.g., over one minute), since the distance between transmitter and receiver in most situations does not change significantly on smaller time scales. In the same way shadowing can be considered constant for very short time scales in the order of few seconds. Thanks to the modular architecture of the DVB-H system-level simulator developed in this thesis, it can be easily configured to perform simulations taking into account only fast fading, or only fast fading and shadowing. 


\section{A.3 DVB-H Physical Layer Performance Model}

The most basic performance model that we could think of is an ON-OFF model, motivated by the rapid transition between near perfect reception to no reception at all characteristic of the underlying DVB-T standard with the received signal strength. However this fast transition only applies for static reception conditions. Under mobility conditions, the performance degradation at the physical layer with fast fading due to the very short time interleaving employed enlarges the CNR dynamic range up to 10 to $15 \mathrm{~dB}$ [52]. Looking at the measured error pattern in Figure A.3, we can clearly see that the assumption of a hard signal strength threshold is not valid in DVB-H. Another alternative would be to model the average packet error rate as a function of the CNR and Doppler, obtaining some kind of look-up tables. This approach is commonly employed in cellular system-level simulations, but it is not practical in our case as the effect of fast fading on the packet error statistics would be lost.

In this dissertation we consider a DVB-H physical layer performance model based on parameterized 4-state aggregated Markov processes originally proposed in [95]. The model approximates the distributions of lengths of sequences of correctly and erroneously received TS packets, matching not only the average packet error rate but also the first order statistics of run length sequences (i.e., the mean error burst and error gap lengths and their respective variances). The term run length is used to refer to the length of a sequence of consecutive erroneous or error-free received packets. In short, the model is based on initially determining - either from laboratory measurements or simulations - relevant run length statistics for a given DVB-H transmission mode and channel model. Then the measured/simulated statistics are parameterized as a function of the CNR and maximum Doppler frequency.

In the following, the structure and parameterization of the model are outlined. To facilitate the implementation of the model, straightforward procedures are given for calculating the model parameters. A more general description of the class of models from which the adopted model is obtained can be found in [96]. In the reference it is also shown that results obtained with a four-state model are not significantly improved by increasing the number of states.

\section{A.3.1 Model Structure}

The state transition probability matrix of the four-state Markov model can be written as:

$$
\left(\begin{array}{cccc}
\alpha_{1} & 0 & \left(1-\alpha_{1}\right) w_{3} & \left(1-\alpha_{1}\right) w_{4} \\
0 & \alpha_{2} & \left(1-\alpha_{2}\right) w_{3} & \left(1-\alpha_{2}\right) w_{4} \\
\left(1-\alpha_{3}\right) w_{1} & \left(1-\alpha_{3}\right) w_{2} & \alpha_{3} & 0 \\
\left(1-\alpha_{4}\right) w_{1} & \left(1-\alpha_{4}\right) w_{2} & 0 & \alpha_{4}
\end{array}\right),
$$

where $0 \leq \alpha_{i} \leq 1, \forall i, w_{1}+w_{2}=1$ and $w_{3}+w_{4}=1$. Each state transition corresponds to one received packet. Transitions ending in states 1 and 2 are associated to the erroneous reception of TS packets, whereas states 3 and 4 correspond to correct reception of TS packets. 


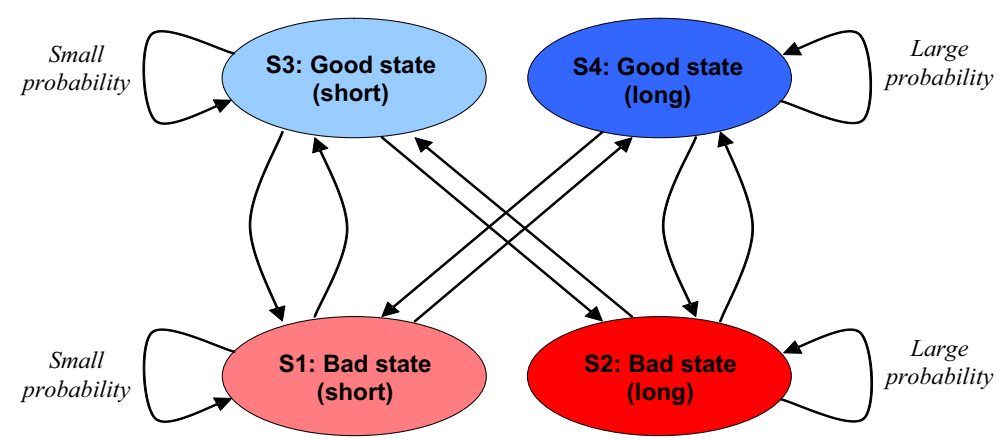

Figure A.8: State diagram of the four-state error model. The good states correspond to correct reception of a TS packet, and the bad states to erroneous reception.

Figure A.8 shows a conceptual state diagram of the error model with qualitative probabilities for staying in the states. Statistical properties of the model may be derived as given in [95]. The run length statistics considered in the model are the following. The packet error probability is defined as:

$$
p_{E}=\frac{w_{1}}{\left(1-\alpha_{1}\right)\left(\sum_{k=1}^{4} \frac{w_{k}}{1-\alpha_{k}}\right)}+\frac{w_{2}}{\left(1-\alpha_{3}\right)\left(\sum_{k=1}^{4} \frac{w_{k}}{1-\alpha_{k}}\right)} .
$$

The mean length of sequences of erroneously received packets is:

$$
\mu_{E}=\frac{w_{1}}{\left(1-\alpha_{1}\right)}+\frac{w_{2}}{\left(1-\alpha_{2}\right)}
$$

the mean length of sequences of correctly received packets:

$$
\mu_{C}=\frac{w_{3}}{\left(1-\alpha_{3}\right)}+\frac{w_{4}}{\left(1-\alpha_{4}\right)}
$$

the variance of lengths of sequences of erroneously received packets:

$$
\sigma_{E}^{2}=\frac{w_{1} \alpha_{1}\left(1-\alpha_{2}\right)^{2}+w_{2} \alpha_{2}\left(1-\alpha_{1}\right)^{2}+w_{1} w_{2}\left(\alpha_{2}-\alpha_{1}\right)^{2}}{\left(1-\alpha_{1}\right)^{2}\left(1-\alpha_{2}\right)^{2}}
$$

and finally the variance of lengths of sequences of correctly received packets:

$$
\sigma_{C}^{2}=\frac{w_{3} \alpha_{3}\left(1-\alpha_{4}\right)^{2}+w_{4} \alpha_{4}\left(1-\alpha_{3}\right)^{2}+w_{3} w_{4}\left(\alpha_{4}-\alpha_{3}\right)^{2}}{\left(1-\alpha_{3}\right)^{2}\left(1-\alpha_{4}\right)^{2}}
$$

The model parameters $a_{i}, w_{1}$ and $w_{3}$ can be obtained by solving numerically two nonlinear systems of equations given by (A.7) and ( A.9), and (A.8) and ( A.9) respectively $\left(w_{2}=1-w_{1} ; w_{4}=1-w_{3}\right)$. Note that this differs from the results provided in [95], where a fifth equation defining the packet error probability was included. This equation is redundant, and removing it was found to improve the speed and accuracy of numerically solving the model parameters. 


\section{A.3.2 Model Implementation}

\section{Measure Run Length Statistics}

First of all, it is necessary to obtain the run length statistics of interest for a given DVB-H physical layer transmission mode and channel model for a relevant range of average CNR $(\rho)$ and maximum Doppler frequency shifts $\left(f_{d}\right)$. This can be done either with physical layer simulations or laboratory measurements. Lab measurements have the intrinsic benefit of characterizing the receiver performance into the model. Note that measurements should be performed separately for each DVB-H transmission mode and channel model considered.

Each measurement corresponding to a fixed (CNR; Doppler)-pair should be long enough to provide consistent estimates of the required packet error statistics. The necessary measurement length depends on the CNR and Doppler values. It can be estimated, for example, using the runs test as described in [95]. In short, the principle is to divide the measured error trace into segments of equal lengths, compute the lengths of runs in each segment, count the number of runs of length above and below the median value for run lengths in the trace, and finally compute a histogram for the number of runs counted. A trace is considered to be valid when the number of runs between the 0.05 and 0.95 cutoffs is close to $90 \%$. In reference [95] it is shown that measurement lengths of order $10^{6}$ TS packets are sufficient for TU6 channel model for the mode FFT 8K GI 1/4 16-QAM 1/2.

Another important issue is how extensive should be the set of measurements corresponding to different CNR and Doppler frequency values. As we are interested in modeling the transition between perfect reception to no reception, it is possible to measure the relevant $\mathrm{CNR}$ range (for example in $1 \mathrm{~dB}$ steps). However, due to the parameterization employed to interpolate the packet error statistics within the measured range, it is also possible to extrapolate the necessary statistics beyond the measured CNR range, reducing the number of CNR points necessary to be measured. The Doppler range should be measured according to the expected values (in $5-10 \mathrm{~Hz}$ intervals, for example). We will show that a relatively small CNR range of $5 \mathrm{~dB}$ with $1 \mathrm{~dB}$ intervals may already produce accurate simulation results compared to field measurements in diverse reception conditions [97].

\section{Parameterization}

The goal of parameterizing the model is to obtain relationships between the measured packet error statistics and the physical reception conditions described by the CNR and the Doppler frequency. The measured statistics are approximated as functions of the CNR and Doppler using planar least-squared error (LSE) approximation in the logarithmic scale. Table A.2 gives the coefficients obtained for the Nokia DVB-H prototype receivers employed in the field measurements in Turku for the physical layer transmission mode FFT 8K GI 1/4 16-QAM 1/2 and TU6 channel model. Lab measurements were performed with CNR ranging from 14 to $18 \mathrm{~dB}$ in $1 \mathrm{~dB}$ steps, Doppler from 5 to $80 \mathrm{~Hz}$ in $5 \mathrm{~Hz}$ steps, and of order $10^{6}$ TS packets per measurement. Approximated values for the relevant packet error statistics as functions of the reception conditions, denoted here jointly by $\tilde{m}\left(\rho ; f_{d}\right)$, can be calculated from the given coefficient values as: $\tilde{m}\left(\rho ; f_{d}\right)=\exp \left(c_{1} \cdot f_{d}+c_{2} \cdot \rho+c_{3}\right)$. 
Table A.2: TU6 model LSE coefficients.

\begin{tabular}{|c||c|c|c|c|}
\hline$\tilde{m}$ & $\mu_{E}$ & $\sigma_{E}^{2}$ & $\mu_{C}$ & $\sigma_{C}^{2}$ \\
\hline \hline$c_{1}$ & $-2.1 \cdot 10^{-3}$ & $-31.2 \cdot 10^{-3}$ & $-2.5 \cdot 10^{-3}$ & $-74.3 \cdot 10^{-3}$ \\
\hline$c_{2}$ & -0.1271 & -0.3467 & 0.5315 & 1.0474 \\
\hline$c_{3}$ & 4.7285 & 12.7569 & -2.1663 & -1.9857 \\
\hline
\end{tabular}

\section{A.3.3 Model Application in the System-Level Simulator}

Let $N_{T S}$ be the number of TS packets per burst that depends on the burst size (maximum size $2 \mathrm{Mb}$ ). Assuming that the mean signal strength (considering path loss and shadowing) and receiver speed are stationary during the burst duration (with typical durations 0.2-0.4 s), error traces are generated using the proposed model by repeating the following steps for each of the simulated users:

1. Calculate the CNR and Doppler values corresponding to the given signal strength and vehicle speed.

2. Using the LSE estimation results as described in the previous section, determine the values of the packet error statistics needed to solve the finite-state model parameters.

3. Calculate the model parameters for the four-state model solving the two systems of equations (A6-A9).

4. Generate $N_{T S}$ output symbols. The initial state of the four-state model can be derived from the theoretical limiting state probabilities, which can be written as [95]:

$$
\pi_{i}=\frac{w_{i}}{\left(1-\alpha_{i}\right)\left(\sum_{k=1}^{4} \frac{w_{k}}{1-\alpha_{i}}\right)} .
$$

Steps 2 and 3 can be replaced by pre-calculating the model parameters for the relevant range of CNR and Doppler to speed up the simulation process.

\section{A.3.4 Model Validation with Laboratory Measurements}

The accuracy of the error model can be verified with the difference between the run length statistics measured and simulated. Here we provide illustrative validation results for the NOKIA prototype receivers where we compare the average TS packet error rate (PER) as a function of the CNR for different maximum Doppler frequencies, and the sample distribution of error burst lengths of one particular measurement, see Figure A.9. The simulation results indicate that the proposed model produces a good fit to the measured average packet error rate and error burst length distribution. 

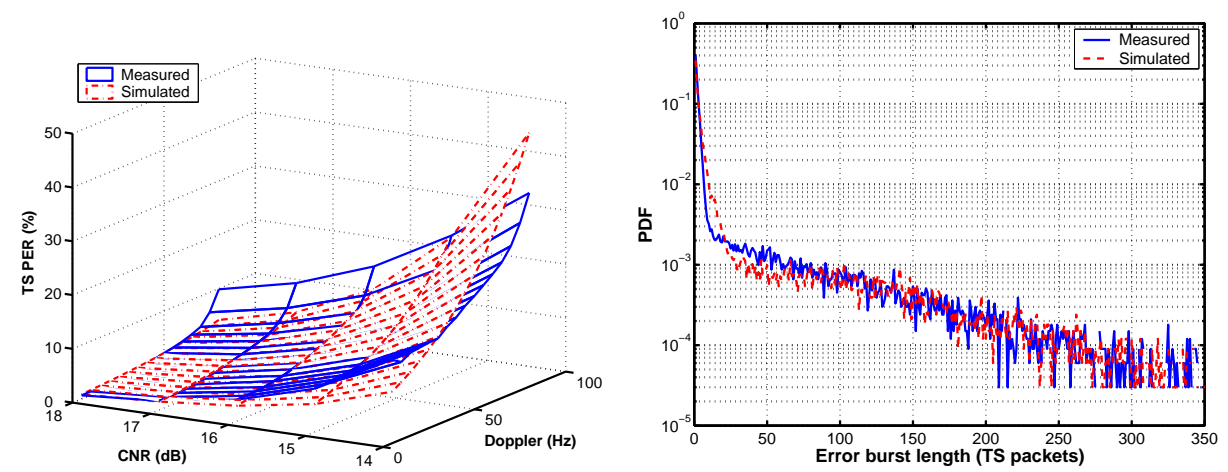

Figure A.9: Comparison between lab measurements and simulations: average TS packet error rate (left) and error burst length for CNR $17 \mathrm{~dB}$ and Doppler $10 \mathrm{~Hz}$ (right).

\section{A.3.5 Model Validation with Field Measurements}

The suitability of the proposed model was also validated with vehicular field measurements by comparing the measured TS error traces to those obtained from simulations using the measured RSSI and vehicle speed values as inputs to the model. CNR values were calculated from the measured RSSI values assuming a constant background noise level and using receiver-specific calibration to obtain a conversion coefficient between RSSI and CNR. In [95] the accuracy of the model was preliminary verified with field measurements performed within the Wing TV project in the DVB-H pilot network in the Hague, the Netherlands. However, only one single six-minute trace was considered. Here we validate the model with a more extensive set of measurements at various locations and transitions around the city of Turku. The total measurement time was 2 hours (approximately $49 \cdot 10^{6}$ MPEG-2 TS packets), divided into 20 individual measurements of length 6 minutes each.

\section{Measured RSSI and Velocity Estimation Analysis}

Figure A.10 shows an example of measured and simulated TS packet error rates (PER) over time for the measured trace shown in Figure A.3(a). Error rates are averaged over $1 \mathrm{~s}$ intervals. It can be seen that the time-variant packet error rate of the simulated error trace follows very closely the measurement.

Table A.3 compares the average statistics for the 2 hours of measurements in terms of TS PER, MPE section error rate (SER), MPE-FEC frame error rate (MFER), IP packet error rate (IP PER), erroneous second ratio (ESR) and ESR5(20). It has been assumed a constant IP packet size 512 bytes and MPE-FEC code rate 3/4 with $1 \mathrm{Mb}$ burst size. In the table we can see how errors propagate in the upper layers of the protocol stack (e.g., MPE SER is higher than TS PER), and that the simulator provides slightly optimistic results compared to the measurements. From these validation results it can be concluded that the accuracy of the proposed DVB-H physical layer performance model is good with measured mobility and coverage information. 


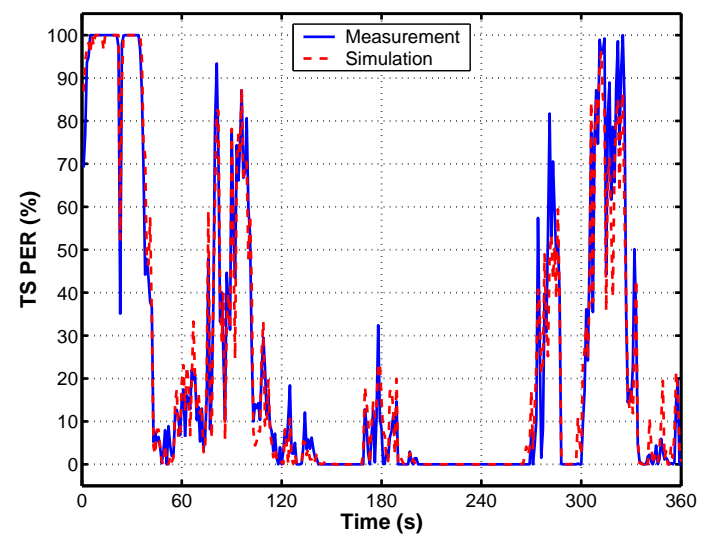

Figure A.10: Dynamic packet error simulation using measured time-dependent RSSI and vehicle speed values.

\begin{tabular}{|c||c|c|c|c|c|c|}
\hline Trace & TS PER & MFER & MPE SER & IP PER & ESR & ESR5(20) \\
\hline \hline Measured & 3.9 & 4.2 & 4.9 & 3.7 & 5.1 & 11.9 \\
\hline Simulated & 3.7 & 3.8 & 4.6 & 3.3 & 4.5 & 11.5 \\
\hline
\end{tabular}

Table A.3: Simulated average error rates (\%) using measured RSSI and speed information.

\section{Imperfect RSSI and Velocity Estimation}

We also investigated the influence of the degree of accuracy of the RSSI and speed estimation on the simulation results, as it would be unrealistic to assume that the mobility and radio coverage models used in the system-level simulator provide ideal information on the time-variant reception conditions when compared to measurements. We found that the DVB-H radio coverage module is a critical element in the system-level simulator. As the physical layer of DVB-H is characterized by a very rapid transition from near perfect reception to no reception at all, an accurate coverage prediction is crucial in order to evaluate which information is correctly received by the users. However, as the aim of system-level simulations is to evaluate the overall QoS perceived by the users statistically, results obtained with a calibrated coverage map may reflect quite accurately the network performance [97]. Recall that the goal of the calibration process is to provide a zero mean and a minimum standard deviation of the error between the prediction and the measurements. During the field measurement campaign this assumption was validated using the RSSI measurements recorded and the coverage map used in Figure A.3.

On the other hand, it was shown that the effect of the speed on the simulation performance is relatively small compared to the effect of adding error in the RSSI estimation if the receiver speed is kept within a realistic range. 


\section{A.4 Cellular System Models}

In this section we describe the system models adopted in the MBMS/HSDPA cellular radio network simulations utilized in Chapter 5.

\section{Deployment Scenario}

For the shake of simplicity, a synthetic scenario has been considered. The network consists of a homogeneous hexagonal lattice of cells of $866 \mathrm{~m}$ radius (the inter-site distance is 1.5 $\mathrm{km})$. No cell sectorization is considered and omnidirectional antennas have been assumed at the base stations.

\section{Link Budget}

Link budget values corresponding to an urban scenario at a frequency of $2 \mathrm{GHz}$ have been considered. We assume lognormal shadowing with a standard deviation of $8 \mathrm{~dB}$ and a correlation distance of $50 \mathrm{~m}$ (correlation factor between cells is 0.5). Fast fading (Rayleigh distributed) is also considered. The distance dependent path loss has been modeled with an urban Okumura-Hata propagation model [123]. It is computed as:

$$
L_{d}=15.3+37.6 \cdot \log _{10}[d] \text {, }
$$

where $d$ is the distance in meters. The thermal noise power at the terminals is $-99 \mathrm{dBm}$, which are modeled with an omnidirectional antenna of $0 \mathrm{dBi}$ gain. The maximum transmission power per cell is $20 \mathrm{~W}$, and all simulated cells transmit with the same power.

\section{Interference Model}

In the simulations, both intra and inter-cell interferences are considered. Two interfering rings are considered. A constant orthogonality factor is used to characterize the interference between channels of the same cell. The performance of MBMS and HSDPA has been tested under full background load conditions, assuming that all base stations transmit at the maximum power of $20 \mathrm{~W}$, modeling a worst-case interference scenario. Other services than the service under study are simulated to contribute with intra-cell interference. The average signal-to-interference plus noise ratio (SINR) during a TTI for user i and base station $\mathrm{j}$ is computed as:

$$
\overline{\operatorname{SINR}}_{i, j}=\frac{S_{\text {useful }}}{I_{\text {intra }}+I_{\text {inter }}+P_{n}},
$$

where $S_{\text {useful }}$ is the useful received power, $I_{\text {intra }}$ and $I_{\text {inter }}$ are the intra-cell and inter-cell interference levels, and $P_{n}$ is the thermal noise. They are computed as:

$$
\begin{aligned}
S_{\text {useful }} & =g_{i, j} \cdot P_{U, j} . \\
I_{\text {intra }} & =(1-\rho) \cdot g_{i, j} \cdot\left(P_{T, j}-P_{U, j}\right) . \\
I_{\text {inter }} & =\sum_{k \neq j} g_{i, k} \cdot P_{T, k} .
\end{aligned}
$$


Where $g_{i, j}$ is the path gain (inverse of the path loss) between user $i$ and base station $j$ including shadowing, $P_{U, j}$ is the transmitted power of the desired channel, $\rho$ is the orthogonality factor (the value employed in the simulations is $\rho=0.6$ ), and $P_{T, j}$ is the total transmitted power of base station $j$. It should be pointed out that the spreading factor of the signal also affects the average SINR, but in our case it is included in the radio link performance model described next.

\section{Radio Link Performance Model}

The radio link performance model is based on a shifted version of the Shannon limit, as proposed in [146]. The maximum data rate, in $\mathrm{bps} / \mathrm{Hz}$, that can be achieved for a given SINR can be computed as:

$$
R_{b}=\log _{2}[1+\gamma \cdot S I N R],
$$

where $0<\gamma \leq 1$ is a degradation term, which shifts the link performance away from the Shannon limit. The effective data rate is obtained from multiplying the data rate with the amount of spectrum utilized for the transmission ( $5 \mathrm{MHz}$ in our case). In our simulations we have chosen a value of $\gamma=0.4$ ( $4 \mathrm{~dB}$ degradation from Shannon limit and assumption of BER $10^{-4}$ [147]).

\section{MBMS and HSDPA Performance Models}

In the simulations we compute the path losses including shadowing every $80 \mathrm{~ms}$ (MBMS TTI period), and the fast fading every $2 \mathrm{~ms}$ (HSDPA TTI period). In the MBMS simulations we compute the number of correctly received TTIs by each user. We first compute the average SINR considering path loss and shadowing in the TTI, and the fast fading experienced by each $2 \mathrm{~ms}$ slot. Then we compute the effective SINR in the TTI using the ECM method (equivalent SNR method based on convex metric) [76]. To decide whether the TTI is correctly received or not, we compare the effective SINR value to the threshold given by Equation A.15 for the bearer data rate employed. For the sake of simplicity we have assumed that one Raptor-coded packet is transmitted per TTI.

In the HSDPA simulations we compute the instantaneous SINR every $2 \mathrm{~ms}$ TTI (average SINR plus fast fading), and we assume that the effective data rate given by Equation A.15 is always correctly received (i.e., ideal link adaptation without retransmissions of lost packets). We consider a minimum data rate equal to $80 \mathrm{~kb} / \mathrm{s}$ (minimum that can be provided in HSDPA), meaning that users can be in outage, and a maximum data rate equal to $3.6 \mathrm{Mb} / \mathrm{s}$. 


\section{A.5 Conclusions}

In this appendix we have discussed the need of complementing the conventional static approach used for analyzing terrestrial digital TV broadcasting networks with dynamic analysis in DVB-H, as the actual service quality perceived by the users depends on the time evolution of the transmission errors suffered.

We have also motivated the need for dynamic DVB-H system-level simulations, in order to evaluate the overall system performance perceived by the users statistically giving a better understanding of the network behavior. Basically, the goal of the simulator is to accurately predict which data is correctly received in each burst for each user moving across a service area. Moreover, this should be achieved in a computationally efficient manner to enable simulation of large groups of users. These simulators can be integrated with conventional DVB-H coverage planning tools, being possible for example to evaluate the influence of additional sites not only in terms of area coverage, but also in terms of service quality perceived by the users. Another important application is to optimize the transmission configuration.

The main models required to perform such simulations in DVB-H have been outlined, and a possible architecture for a system-level simulator has been described. A mobility model moves users across the service area and computes the speed of the users when receiving a burst, a signal propagation model computes the average received signal strength during the reception of a burst for each user, and finally a DVB-H performance model computes which data is correctly received per burst for each user based on the information of the time-variant reception conditions obtained from the mobility and signal propagation models.

We have considered a parameterized 4-state aggregated Markov process to model the performance of the DVB-H physical layer as a function of the CNR and Doppler for a given transmission mode and channel model. The model approximates the distributions of lengths of sequences of correctly and erroneously received MPEG-2 TS packets, matching not only the average packet error rate but also the first order statistics of run lengths sequences (i.e., the mean error burst and error gap lengths and their respective variances). The model has been validated with laboratory and field measurements, yielding very satisfactory results. We found that good dynamic simulation performance is achieved using the proposed performance model with accurate mobility and radio propagation estimates. Furthermore a relatively small set of lab measurements is required to produce good simulation results compared to field measurements. During the model validation with field measurements it was also found that the coverage prediction is a critical element in the simulator to determine which areas are going to be covered and which are not. 


\section{Appendix B}

\section{Fading Margin Gain due to Multi-Slot FEC in TDM Broadcast Systems}

\section{B.1 Introduction}

In wireless communications mobile users typically experience spatial fading effects due to the superposition of multiple propagation paths between transmitter and receiver. In general it can be distinguished between slow or long-term fading due to local shadowing effects, commonly known as shadowing, and fast or short-term fading due to the coherent superposition of the signals reaching the terminal, commonly known as fast or multipath fading [121], see Appendix A.2.2. Shadowing implies significant variations of several dB over distances of several tens of meters (comparable to the widths of buildings in the region of the mobile), whereas fast fading involves variations on the scale of a half-wavelength and frequently introduces variations as large as 35 to $40 \mathrm{~dB}$.

Signal fading can be compensated with forward error correction (FEC) using an interleaving large enough to essentially average out the fading statistics. FEC mechanisms rely on the transmission of redundant repair information (parity data) such that the receiver can correct errors occurred during the transmission. For the FEC code to be efficient, the different code symbols should ideally experience uncorrelated fading. In this case it is possible to cope with error rates equal to the proportion of parity data transmitted. Otherwise the FEC coding efficiency is reduced.

In case of fast fading, a short time interleaving is required to eliminate it (in the order of few to several milliseconds depending on the user velocity and operating frequency). This is traditionally done at the physical layer as part of the radio interface for wireless communication systems. Physical layer FEC codes work at the bit level and they theoretically provide the most effective protection against channel noise. The reason is that they can exploit channel state information using soft decision decoding where each bit is assigned a confidence value, which provides more reliable decisions. 
This is typically not a feasible way to cope with shadowing due to available memoryon-chip and decoding complexity constraints, especially for handheld devices, since the size of the interleaver would be huge. The memory requirement is directly proportional to the fraction of parity data transmitted, the service data rate and the interleaving duration. Moreover the imposed delay would not be acceptable in many cases, as terminals must wait and store all data jointly encoded before decoding and passing the information to the upper layers. The traditional approach to cope with shadowing consists in adding an extra fading margin of several $\mathrm{dB}$ to the link budget in order to assure a reliable communication with a given probability at the cell edge.

Nevertheless, for mobile users it is in principle possible to reduce the required fading margin using a FEC scheme spanning a sufficiently long time. Recent advances in FEC mechanisms and the continuously increasing computer processing and memory capabilities of handhelds devices make this possibility a reality for services with no real-time requirements. Today newly deployed time division multiplexing (TDM) broadcast systems such as DVB-H [14] and DVB-SH [28] include optional multi-slot FEC schemes in the upper layers to cope with shadowing. Particularly, application layer FEC (AL-FEC) using Raptor coding [55] for file download services in DVB-H [57] and multi protocol encapsulation inter-burst FEC (MPE-iFEC) using sliding Reed-Solomon encoding (SRSE) [81] for streaming services in DVB-SH, see Chapter 2. By operating above the physical layer it is possible to provide protection against longer losses with larger interleaving depths that physical layer cannot. One key reason is that the high memory requirements allow support of host-based storage instead of memory-on-chip. It should be pointed out that upper layer FEC recovers from packet losses using erasure decoding, where each packet is either considered correctly received or lost.

In this appendix we investigate the reduction in the fading margin that can be achieved with multi-slot FEC in a generic TDM broadcast system as a function of the proportion of parity data devoted to cope with shadowing and the interleaving depth in number of slots jointly encoded. The gain will depend on the statistical correlation of the shadowing between consecutive slots. The lower the correlation between reception conditions, the higher the gain. We will also show that the gain depends on the standard deviation of the shadowing and the target service availability. Numerical results will be validated with DVB-H figures. Finally we will also discuss the fading margin required for file download services with and without multi-slot FEC. It should be pointed out that although the problem description has been tailored to broadcast systems results apply for any TDM wireless communication system (unicast or broadcast).

\section{B.2 Problem Description}

We consider a generic TDM broadcast system where the different services are transmitted at the same frequency but in different time-slots. Without loss of generality we assume that the cycle time between slots of the same service, $T_{c}$, is constant. The system is characterized by a receiver sensitivity $P_{s, \min }$, minimum signal level at which reception is possible. 


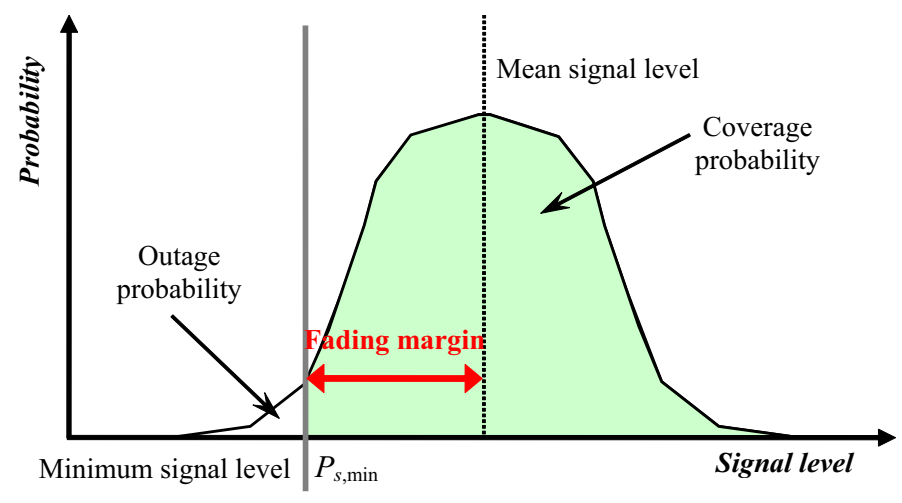

Figure B.1: Shadowing fading margin concept.

Fast fading is assumed to be eliminated at the physical layer with some form of FEC and short time interleaving during the slot duration. Shadowing is assumed to remain constant during this time. It is worth to note that these assumptions are commonly accepted in DVB-H/SH. Their physical layer FEC have a brick-wall characteristic that corrects above a certain signal-to-noise ratio (SNR) threshold whereas fails below; and the slot (time-sliced burst) duration is in the order of few hundreds of milliseconds.

The variations of the shadowing in $\mathrm{dB}$ are described by a zero-mean Gaussian random variable with standard deviation $\sigma_{l}$ and correlation distance $d_{\text {corr }}$ [121]. The shadowing standard deviation is known as the location variability, and it determines the spread of the signal field strength around the mean value. Values typically used for outdoor broadcasting signals are $5.5 \mathrm{~dB}$ in the UHF band and $8 \mathrm{~dB}$ in the $\mathrm{S}$ band [34]. On the other side, the spatial correlation is modeled as a first-order exponential model [120]:

$$
\rho(d)=e^{-d / d_{\text {corr }}},
$$

where $d_{c o r r}$ is distance taken for the autocorrelation to fall by $e^{-1}$.

Shadowing makes coverage prediction statistical, such that it is predicted the signal availability rather than the signal level, see Figure B.1. The outage probability at a single reception point with an average signal level $\overline{P_{s}}$ is equal to the probability that the shadowing increases the median path loss by at least $\overline{P_{s}}-P_{s, \min } \mathrm{dB}$, and is given by:

$$
\text { Outage Probability }=Q\left(\frac{\overline{P_{s}}-P_{s, \min }}{\sigma_{l}}\right) .
$$

Where the $Q(\cdot)$ function is the complementary cumulative normal distribution:

$$
Q(x)=\frac{1}{\sqrt{2 \pi}} \int_{x}^{\infty} e^{-t / 2} d t .
$$

Values for $Q(\cdot)$ can be calculated from the standard cumulative error $(e r f c)$ function. 


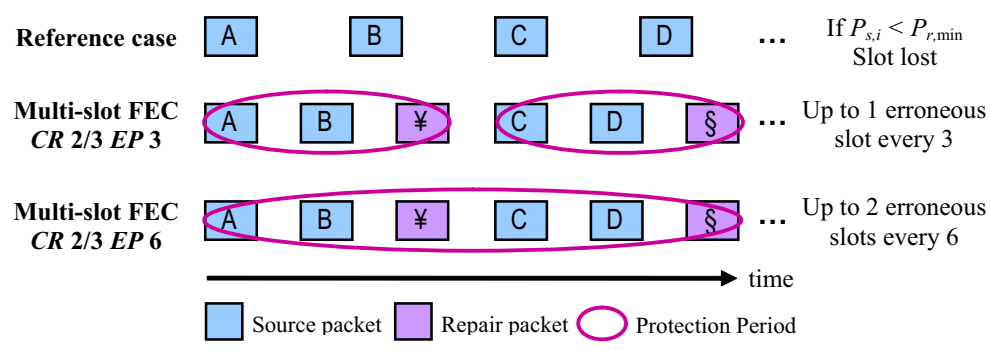

Figure B.2: Multi-slot FEC concept in a generic TDM broadcast system.

The fading margin required to assure a reliable communication with a given probability can be easily computed as:

$$
\text { Fading Margin }=\mu \cdot \sigma_{l}
$$

where $\mu$ is the distribution factor that depends on the coverage probability target. It takes the value of 0.52 for $70 \%, 1.28$ for $90 \%, 1.64$ for $95 \%$, and 2.33 for $99 \%$. Typical targets in DVB-H/SH are $95 \%$ for pedestrian reception and $99 \%$ for vehicular reception [34].

We consider an ideal systematic FEC erasure code operating above the physical layer that produces repair packets to compensate for potential losses due to shadowing, which are sent along with the original source packets. The original multimedia stream is dividing into consecutive source blocks, which are treated and encoded independently. The multislot FEC configuration parameters are the ratio of source information to the total amount of source and repair data transmitted, commonly known as code rate $(C R)$, and the number of slots jointly encoded, here denoted as encoding period $(E P)$. An illustrative example is depicted in Figure B.2. Note that with multi-slot FEC packets are transmitted with a faster data rate to maintain the timing of the original stream.

For the reference case, if the received signal strength is lower than the receiver sensitivity the slot will be lost. With multi-slot FEC it is possible to cope to some extent with temporary slot losses. The error correction capability in terms of percentage of erroneous slots that can be corrected depends only on the code rate. The more repair data is transmitted, the more robust the transmission, at the expense of a decreased system capacity. However the transmission robustness depends also on the encoding period. In general, the longer the interleaving the more robust the transmission, at the expense of an increased latency and larger memory capabilities at the terminals. In the example shown, a code rate $2 / 3$ can correct up to $33 \%$ errors, and thus it will be possible to recover from one erroneous slot every three (for EP 3), two erroneous slots every six (for EP 6) and so on, as soon as all other slots within the encoding period are correctly received.

Multi-slot FEC can reduce the fading margin required to cope with shadowing because the probability of correctly receiving a slot, henceforth denoted as service availability, will be higher than the coverage probability (percentage of covered locations with an average signal level higher than the receiver sensitivity). 


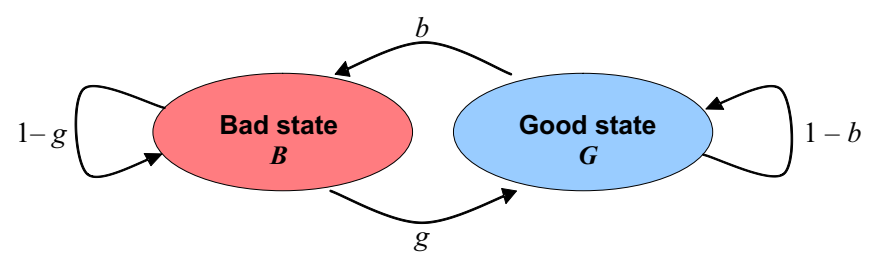

Figure B.3: The Gilbert-Elliott channel.

\section{B.3 Theoretical Analysis with Uncorrelated Shadowing}

\section{Performance Evaluation}

The maximum fading margin gain occurs when there is no shadowing correlation between reception conditions of consecutive slots. In this case the analysis can be derived analytically using the well-known Gilbert-Elliott (GE) channel model. The GE model dates back to the early 60's [148]- [149]. In this model the channel is assumed to be either in a good state where the error rate is small, or in a bad state where the error rate is large. The GE model is depicted in Figure B.3.

The good and the bad states are denoted $G$ and $B$ respectively, and the probabilities that the channel changes from the good state to the bad state and vice versa $b$ and $g$ respectively. The stationary probabilities that the channel is in the good and the bad state are:

$$
P^{\infty}(G)=\frac{g}{g+b} ; P^{\infty}(B)=\frac{b}{g+b} .
$$

In our case, as there is no correlation between consecutive time-slots, the GE channel is memoryless, and the probability of being in the good or bad state is independent of the previous state. The GE channel becomes memoryless when $g+b=1$. We further assume that the probability of erasure is 1 in the bad state and 0 in the good state. Therefore, $g$ and $b$ are the coverage and outage probabilities respectively.

Under these conditions, if the GE channel is observed at $\mathrm{n}$ consecutive instants of time, the probability that the channel is in the bad state $\mathrm{d}$ times, $0 \leq d \leq n$, is given by:

$$
\mathrm{P}_{n}(d)=\left(\begin{array}{l}
n \\
n
\end{array}\right) \cdot g^{(n-d)} \cdot(1-g)^{d} .
$$

With multi-slot FEC, given the code rate and the encoding period, the minimum number of time-slots needed to decode a source data block can be computed as:

$$
S_{\min }=\lceil C R \cdot E P\rceil,
$$

where $\lceil\cdot\rceil$ is the ceiling function. Finally, the service availability, defined as the probability of receiving a time-slot correctly after multi-slot FEC decoding, can be computed as:

$$
\text { Service Availability }=1-\sum_{i=S_{\min }+1}^{E P} \mathrm{P}_{E P}(i) \cdot \frac{i}{E P}
$$




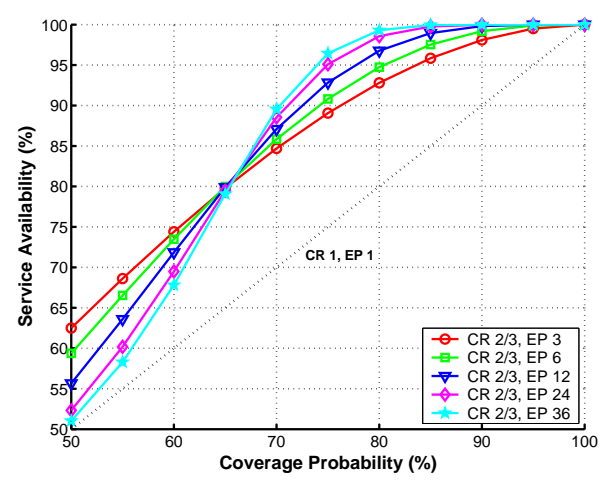

Figure B.4: Service availability vs. Coverage probability.

\section{Analytical Results}

Figure B.4 shows the service availability as a function of the coverage probability for a multi-slot FEC with code rate $2 / 3$ for different encoding periods. The case for $E P 1$ and $C R$ 1 without multi-slot FEC is also shown for comparison. In this case the service availability is equal to the coverage probability.

In the figure we can see how the service availability perceived by the users with multislot FEC is considerably increased compared to the reference case, and that the improvement depends on the interleaving and the coverage probability. It should be noted that increasing the interleaving is only beneficial when the code rate is robust enough to correct the total number of errors (i.e., for coverage probabilities above $66 \%$ in the figure), otherwise the performance may even worsen.

From the results shown in Figure B.4, it can be obtained a new distribution factor for computing the fading margin. The fading margin gain will thus depend on the code rate, interleaving depth, and target service availability, and it will be proportional to the standard deviation of the shadowing. Figure B.5 presents fading margin gain results for different code rates as a function of the interleaving depth for $\sigma_{l} 5.5$ and $95 \%$ service availability. In this case the fading margin without multi-slot FEC would be $9 \mathrm{~dB}$.

We can see that the gain increases for more robust code rates and for longer interleaving. The potential gain is very significant, even for short interleaving, especially for code rates $1 / 2$ and $2 / 3$. Finally we want to point out that the gain also increases for more demanding service probabilities, and for example gain values for $99 \%$ service availability are between $1 \mathrm{~dB}$ and $3 \mathrm{~dB}$ larger than the ones shown in the figure.

\section{B.4 Simulation Analysis with Correlated Shadowing}

\section{Performance Evaluation}

Next, we investigate the fading margin gain due to multi-slot FEC with correlated shadowing. The shadowing correlation between consecutive time-slots depends on the shadowing correlation distance, the cycle time between slots, and the user velocity, see Equation (B.1). 


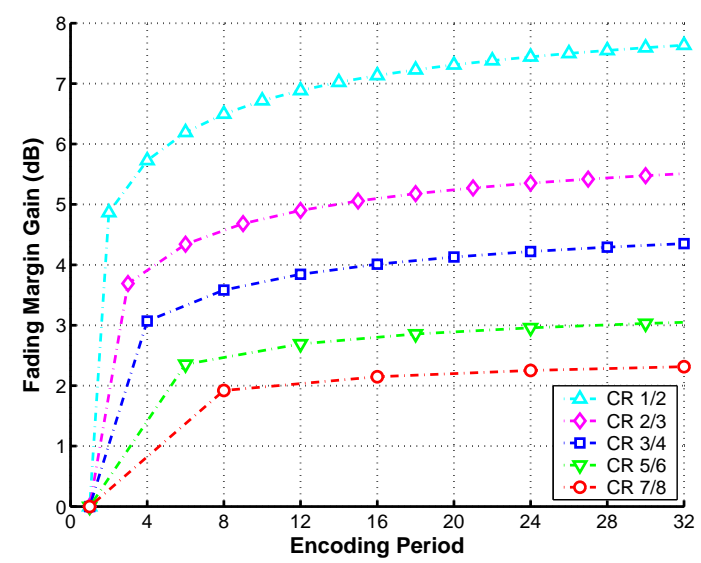

Figure B.5: Fading margin gain for $95 \%$ service availability vs. Encoding period in slot units. Uncorrelated shadowing: $\sigma_{l}=5.5 \mathrm{~dB}, d_{\text {corr }}=0 \mathrm{~m}$.

In general the assumption that the shadowing is uncorrelated between consecutive timeslots will not apply in most cases. For example in DVB-H/SH typical cycle time values for mobile TV streaming services are in the range of one to few seconds, and thus only vehicular users with very high speeds will experience uncorrelated shadowing between consecutive slots.

The gains will be smaller than the ones shown in Figure B.5, and will decrease for larger shadowing correlation distances, smaller user velocities and cycle time values. But it should be clear that the maximum gain does not depend on these parameters, and they basically determine the level of time interleaving required for achieving a given gain. Indeed all three parameters can be combined into one: the ratio between the moved distance by the user during the cycle time and the shadowing correlation distance, $d / d_{c o r r}$.

Although it has been proposed to use the GE model to analytically investigate the effect of correlated shadowing for file download services in DVB-H in [149], it cannot completely capture the exponential correlation property of the shadowing. Therefore, we evaluate the performance of multi-slot FEC under correlated shadowing by means of Monte-Carlo simulations. To simulate correlated shadowing we employ the methodology proposed in [121], and depicted in Figure A.7.

\section{Simulation Results}

Figure B.6 shows the fading margin gain with correlated shadowing as a function of the interleaving depth for $\sigma_{l} 5.5 \mathrm{~dB}, 95 \%$ service availability and code rate $2 / 3$. In the figure we can see how the gain decreases when the correlation between consecutive slots increases. It should be noted that there is no gain by performing a multi-slot protection of the transmission for fully static reception conditions (i.e., $d / d_{\text {corr }}=0$ ), as users will either receive all content or nothing. 


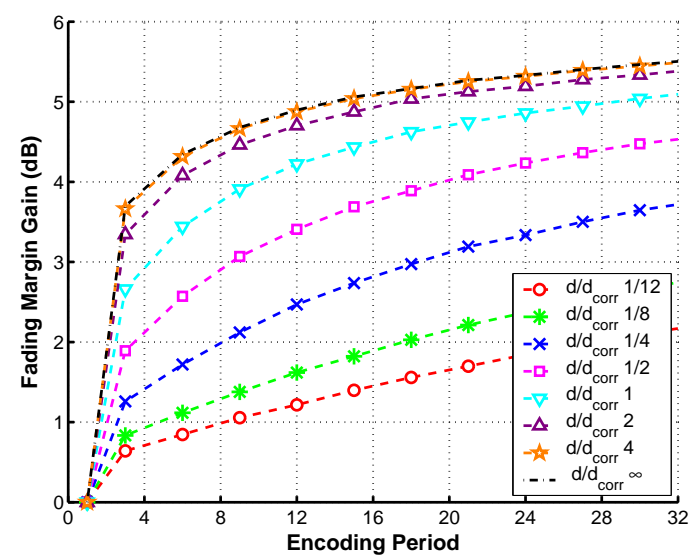

Figure B.6: Fading margin gain vs. Encoding period in slot units. Service availability $95 \%$. Code rate 2/3. Correlated shadowing: $\sigma_{l}=5.5 \mathrm{~dB}$.

The gain due to multi-slot FEC stems from the time diversity of the mobile channel due to the mobility of the users (spatial diversity) and the dynamic variations in the surroundings of the receiving environment. Therefore, for a given scenario and transmission configuration (code rate and encoding period), the gain depends on the user velocity.

\section{B.5 Validation with DVB-H Results}

Figure B.7 compares the theoretical fading margin results shown in Figure B.5 for uncorrelated shadowing (dashed lines) with simulation results for DVB-H (solid lines). We employ the DVB-H physical layer performance model described in Appendix A.3 for the transmission mode: FFT size $8 \mathrm{~K}$, guard interval $1 / 4$, modulation $16-\mathrm{QAM}$ and code rate $1 / 2$. The model was developed from laboratory measurements for the typical urban 6-tap TU6 channel model. This channel model is representative for DVB-H vehicular reception [14], and it includes the time variant small-scale fluctuations of the received signal due to receiver mobility (i.e., fast fading). DVB-H results are for $10 \mathrm{~Hz}$ Doppler and for an ideal multi-slot FEC code in the upper link or application layers (burst size is $2 \mathrm{Mb}$ ).

In the figure we can see that DVB-H results resemble to some extent the theoretical results. The difference is due to the fact that fast fading is not completely eliminated at the DVB-H physical layer due to the very short time interleaving employed (couple of milliseconds maximum) [14]. Hence, the multi-slot FEC code copes not only with completely erroneous time-slots due to shadowing but also with partial received slots due to fast fading. As a consequence some parity data is employed to repair partially received slots, reducing the gain due to shadowing.

Nevertheless, multi-slot FEC also provides a gain due to fast fading in DVB-H, which can even exceed the gain due to shadowing if it is small. This occurs for high code rates, where the percentage of lost slots that can be recovered is rather small (e.g., for a code rate $7 / 8$ only one out of eight slots can be repaired if the remaining seven slots are received 


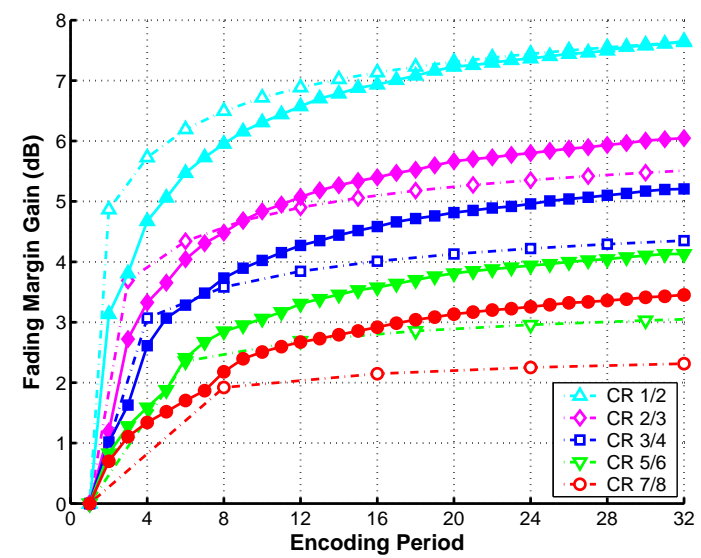

Figure B.7: Fading margin gain for $95 \%$ service availability vs. Encoding period in slot units. Uncorrelated shadowing: $\sigma_{l}=5.5 \mathrm{~dB}, d_{\text {corr }}=0 \mathrm{~m}$. DVB-H FFT 8K GI $1 / 416-\mathrm{QAM} 1 / 2, B_{s}=2 \mathrm{Mb}$, TU6 $f_{d}=10 \mathrm{~Hz}$.

without errors), and when the shadowing correlation between consecutive slots is high. On the other side, for robust code rates such as $1 / 2$ the gain due to shadowing is larger than the gain due to fast fading, and then the theoretical gain due to shadowing is higher than the gain obtained in DVB-H. The difference is larger for shorter interleaving, as the influence of receiving partially a slot is more significant.

\section{B.6 Discussion File Download Services}

Nowadays multimedia content is in general delivered either as a streaming service or as a file download service to the end-users. File download services consist on the delivery of a finite amount of data that is stored into the terminals as a file. Typically, an errorfree reception of the files is required, as even a single bit error can corrupt the whole file and make it useless for the receiver. Hence file delivery in a generic TDM broadcast system without multi-slot FEC implies that each of the unique time-slots where the file is partitioned must be correctly received. Large files spanning several slots burst will be thus more difficult to deliver, as it will be more likely to lose at least one slot.

Assuming that each time-slot experiences uncorrelated shadowing, the probability of successfully receiving the file, denoted as file acquisition probability, can be computed as:

$$
\text { Acquisition Probability }=\mathrm{P}_{F_{s}}(0)=g^{F_{s}} \text {, }
$$

where $g$ is the coverage probability and $F_{S}$ is the file size in number of slots. We can see that the acquisition probability decreases exponentially with the file size. In lossy environments the complete file may not be fully received by most users during the initial file transmission, since some slots may be lost. In these cases one common approach is to repeatedly transmit the file in a carousel. 

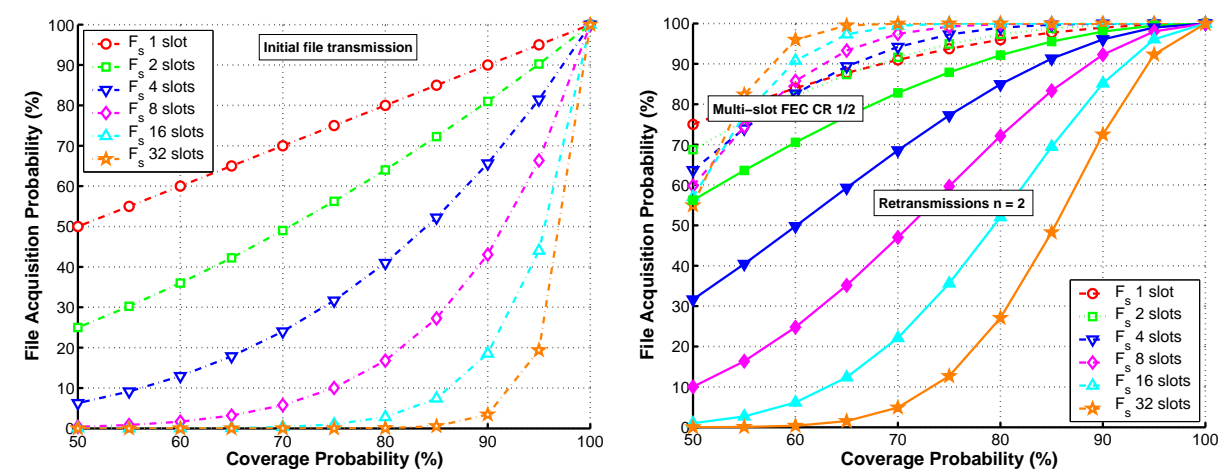

Figure B.8: File acquisition probability vs. Coverage probability.

If the file is transmitted $n$ times, the file acquisition probability becomes:

$$
\text { Acquisition Probability }=\left(1-b^{n}\right)^{F_{s}}
$$

where $b$ is the outage probability. The problem of transmitting the file in a carousel with repetitions is that users may receive duplicate packets which are useless. If a user misses a single packet, he must wait until that specific packet is retransmitted and correctly received, discarding in the mean time packets already received, see Chapter 2.2.3.

In contrast, with multi-slot FEC if a single FEC code word is applied over the entire file all source and repair packets are useful. It makes no difference which packets are received and terminals never receive duplicate data. The file acquisition probability can be computed as:

$$
\text { Acquisition Probability }=\sum_{i=0}^{E P-F_{s}} \mathrm{P}_{E P}(i),
$$

being $E P=F_{S} / C R$ the total number of source and repair slots transmitted. Figure B. 8 shows the file acquisition probability as a function of the coverage probability selected delivery cases for different file sizes. In particular it is shown the reference case without multi-slot FEC and without retransmissions, the case with one file retransmission, and the case with multi-slot FEC with 1/2 code rate. Fading margin values can be obtained from the results shown as explained before for streaming services.

In the figure we can see that without multi-slot FEC and without repetitions an efficient file delivery is only possible within the service area where the coverage probability is close to one. Hence for this delivery case larger fading margins are required than for conventional streaming services. By retransmitting the file the acquisition probability increases, but the number of retransmissions required to achieve a certain high target still increases drastically with the outage probability and the file size. This problem can be solved with multi-slot FEC, which results in very efficient delivery of files. The gain can be translated into a reduction of the transmission time, being possible to deliver more content with the same bandwidth, or into a reduction of the required fading margin. 
The effect of correlated shadowing when multi-slot FEC is employed is the same than for streaming services. That is, the performance (acquisition probability) decreases when the correlation increases. File retransmissions also suffer a similar degradation. However this is not true for the initial file transmission without multi-slot FEC, as in this case the acquisition probability increases with the correlation towards the coverage probability.

\section{B.7 Conclusions}

In this appendix we have evaluated the fading margin gain that can be achieved with multislot FEC in a generic TDM wireless system as a function of the number of slots jointly encoded and the code rate devoted to cope with shadowing. Gain values have been derived analytically for the case with uncorrelated shadowing between consecutive slots and with simulations for the case with correlated shadowing.

Multi-slot FEC exploits the time diversity of the mobile channel to recover from temporary losses, increasing the service availability compared to the coverage probability. Hence, for a target service availability level, the required coverage probability is reduced, which yields a lower fading margin.

The gain increases for more robust code rates, for longer interleaving and for more demanding service availabilities, and it is directly proportional to the shadowing standard deviation. The potential gain is very significant for robust code rates such as $1 / 2$ or $2 / 3$, even for short interleaving depths. But the gain depends on the statistical correlation of the shadowing between consecutive slots. The lower the correlation, the higher the gain. For a given scenario and transmission configuration, the gain will depend on the user velocity. Multi-slot FEC is thus especially suited for vehicular users due to their high speeds, whereas lower gains can be expected for pedestrian users due to their reduced mobility. In any case very important gains are feasible for background services where a relatively large latency is not an issue, as it is possible to encode large amounts of slots jointly or to reduce the correlation level between reception conditions of consecutive slots by increasing the cycle time between slots. File download services fall in this category of services, and they can in general fully exploit the benefits of multi-slot FEC. On the other hand for streaming services it is necessary to establish an adequate trade-off between the protection provided by multi-slot FEC and the latency introduced in the system, as it may compromise the user experience. 



\section{Bibliography}

[1] S. Orgad, "This Box Was Made for Walking ... How Will Mobile Television Transform Viewers Experience and Change Advertising?," Nokia white paper, Nov. 2006.

[2] Y. Solomon, “The Economics of Mobile Broadcast TV," Mobile DTV Alliance whitepaper, Jan. 2007.

[3] H. Holma and A. Toskala, "HSDPA/HSUPA for UMTS: High Speed Radio Access for Mobile Communications," Wiley, 2006.

[4] H. Ekström et al., "Technical Solutions for the 3G Long-Term Evolution," IEEE Communications Magazine, vol. 44, no. 3, pp. 38-46, March 2006.

[5] S. Uskela, "Key Concepts for Evolution toward Beyond 3G Networks," IEEE Wireless Communications, vol. 10, no. 1, pp. 43-48, Feb. 2003.

[6] B. Karlson, A. Bria, J. Lind, P. Lönnqvist, and C. Norlin, "Wireless Foresight: Scenarios of the Mobile World in 2015," Wiley, 2003.

[7] S. Mason, "Mobile TV - Results from the DVB-H Trial in Oxford," EBU Technical Review, April 2006.

[8] E. Lloyd, R. Maclean, and A. Stirling, "Mobile TV - Results from the BT Movio DAB-IP pilot in London," EBU Technical Review, April 2006.

[9] A. Bria, P. Kärrberg, and P. Andersson, "TV in the Mobile or TV for the Mobile: Challenges and Changing Value Chains," Proc. IEEE PIMRC, Athens, Greece, 2007.

[10] R. Walsh, A. P. Vainio, and J. Aaltonen, "Multicast Content Delivery for Mobiles," Content Networking in the Mobile Internet, Wiley, 2004.

[11] F. Hartung et al. "Delivery of Broadcast Services in 3G Networks," IEEE Trans. on Broadcasting, vol. 52., no. 1, pp. 188-199, March 2007.

[12] L. Ardito and C. Sattler, "Mobile Broadcast Bearer Technologies - a Comparison," BMCO forum whitepaper, Feb. 2009.

[13] M. Kornfeld and U. Reimers, "DVB-H - The Emerging Standard for Mobile Data Communication," EBU Technical Review, Jan. 2005. 
[14] G. Faria, J. A. Henriksson, E. Stare, and P. Talmola, "DVB-H: Digital Broadcast Services to Handheld Devices," Proc. of the IEEE, vol. 94, no. 1, Jan. 2006, pp. 194-209.

[15] V. Reading, "Digital TV, Mobile TV: Let's Push for Open Technologies in Europe and Worldwide," DVB World Conference, Budapest, Hungary, 2008.

[16] 3GPP TS 25.346 v8.3.0, "Introduction of the Multimedia Broadcast Multicast Service (MBMS) in the Radio Access Network (RAN); Stage 2," March 2009.

[17] S. Cho et al., "System and Services of Terrestrial Digital Multimedia Broadcasting (TDMB)," IEEE Trans. on Broadcasting, vol. 52., no. 1, pp. 171-178, March 2007.

[18] G. Bedicks and C. Akamine, "Overview of ISDB-T: One-Segment Reception," Handbook of Mobile Broadcasting: DVB-H, DMB, ISDB-T and Media FLO, CRC Press, 2008.

[19] M. R. Chari et al., "FLO Physical Layer: An Overview," IEEE Trans. on Broadcasting, vol. 52., no. 1, pp. 145-160, March 2007.

[20] U. Ladebusch and C. Liss, "Terrestrial DVB (DVB-T): A Broadcast Technology for Stationary Portable and Mobile Use," Proc. of the IEEE, vol. 94, no. 1, pp. 183-193, Jan. 2006.

[21] J. H. Stott, "The How and Why of CODFM," EBU Technical Review, Winter 1998.

[22] M. Kornfeld and G. May, "DVB-H and IP Datacast - Broadcast to Handheld Devices," IEEE Trans. on Broadcasting, vol. 53, no. 1, pp. 161-170, March 2007.

[23] W. Tuttlebee, D. Babb, J. Irvine, G. Martinez and K. Worrall, "Broadcasting and Mobile Telecommunications: Interworking not Convergence,” EBU Technical Review, Jan. 2003.

[24] N. Niebert, A. Schieder, J. Zander, and R. Hancock, "Ambient Networks: Co-operative Mobile Networking for the Wireless World," Wiley, 2007.

[25] J. Zander, "Affordable Multiservice Wireless Networks - Research Challenges for the Next Decade," Proc. IEEE PIMRC, Lisbon, Portugal, 2002.

[26] J. Zander, "On the Cost Structure of Future Wideband Wireless Access," Proc. IEEE VTC Spring, Phoenix, USA, 1997.

[27] A. Hoikkanen, "Economics of Wireless Broadcasting over DVB-H Networks," Proc. IEEE WTS, Ponoma, USA, 2006.

[28] P. Kelley and C. Rigal, "DVB-SH - Mobile Digital TV in S band," EBU Technical Review, July 2007.

[29] J. R. Bughin, "Understanding Europe's Market for Mobile TV," The McKinsey Quarterly, April 2006.

[30] C. Sattler, "Mobile Broadcast Business Models - A State of the Art Study," BMCO forum white paper, Nov. 2006.

[31] H. Mittermayr and C. Sattler, "Mobile Broadcast Business Models - Country-specific Implementations," BMCO forum whitepaper, Feb. 2009. 
[32] C. Sattler, "Best Practice Regulatory Framework for Mobile TV," BMCO forum white paper, June 2008 .

[33] ETSI TR 101190 v1.2.1, "Digital Video Broadcasting (DVB); Implementation Guidelines for DVB Terrestrial Services; Transmission Aspects," Nov. 2004.

[34] J.-J. Delmas and P. Bretillon, "Mobile Broadcast Technologies - Link Budgets," BMCO forum white paper, Feb. 2009.

[35] ICNIRP Guidelines, "Guidelines for Limiting Exposure to Time-Varying Electric, Magnetic, and Electromagnetic Fields (up to $300 \mathrm{GHz}$ )," Health Physics, vol. 87, no. 2, pp. 171-186, Aug. 2004.

[36] D. Gómez-Barquero and A. Bria, "Feasibility of DVB-H Deployment on Existing Wireless Infrastructure," Proc. IWCT, Oulu, Finland, 2005.

[37] A. Bria and D. Gómez-Barquero, "Scalability of DVB-H Deployment on Existing Wireless Infrastructure," Proc. IEEE PIMRC, Berlin, Germany, 2005.

[38] D. Gómez-Barquero, A. Bria, J. Zander, and N. Cardona, "Affordable Mobile TV Services in Hybrid Cellular and DVB-H Systems," IEEE Network, vol. 21, no. 2, pp. 34-40, March-April 2007.

[39] J. Zander, "Competitive Wireless Multi-Access - Implications and Research Issues," Proc. IEEE PIMRC, Helsinki, Finland, 2006.

[40] D. Gómez-Barquero, A. Bria, J. F. Monserrat, and N. Cardona, "Minimal Cost Planning of DVB-H Networks on Existing Wireless Infrastructure," Proc. IEEE PIMRC, Helsinki, Finland, 2006.

[41] R. Keller, T. Lohmar, R. Tonjes, and J. Thielecke, "Convergence of Cellular and Broadcast Networks from a Multi-Radio Perspective," IEEE Wireless Communications, vol. 8, no. 2, pp. 51-56, April 2001.

[42] S. Parkvall, E. Englund, M. Lundevall, and J. Torsner, "Evolving 3G Mobile Systems: Broadband and Broadcast Services in WCDMA," IEEE Communications Magazine, vol. 44, no. 2, pp. 68-74, Feb. 2006.

[43] P. Unger and T. Kürner, "Radio Network Planning of DVB-H/UMTS Hybrid Mobile Communication Networks," Wiley European Transactions on Telecommunication, vol. 17 no. 2, pp. 193-201, March 2006.

[44] D. Gómez-Barquero and A. Bria, "Error Repair for Broadcast Transmissions in DVB-H Systems," Wiley Wireless Communications and Mobile Computing, vol. 9, no. 6, pp. 733-744, June 2009.

[45] A. Bria, "Mobile Multimedia Multicasting in Future Wireless Systems - A Hybrid Cellular and Broadcasting System Approach," Ph.D. Thesis, Royal Institute of Technology, Sweden, 2009.

[46] DVB draft bluebook, "Upper Layer Forward Error Correction in DVB," work in progress. 
[47] Y. Zhang, J. Cosmas, M. Bard, and Y.-H. Song, "Diversity Gain for DVB-H by using Transmitter/Receiver Cyclic Delay Diversity," IEEE Trans. on Broadcasting, vol. 52, no. 4, pp. 464-474, Dec. 2006.

[48] M. Lattuada, R. Posega, M. Marco, D. Mlinek, "Improving DVB-T Forward Error Correction by Concatenated Turbo Code Scheme,” Proc. Packet Video Workshop, Nantes, France, 2003.

[49] T. Jokela, "Performance Analysis of Substituting DVB-S2 LDPC Code for DVB-T Error Control Coding System," Proc. IEEE BMSB, Las Vegas, USA, 2008.

[50] J. Paavola et al., "The Performance Analysis of MPE-FEC Decoding Methods at the DVB-H Link Layer for Efficient IP Packet Retrieval,” IEEE Trans. on Broadcasting, vol. 53, no. 1, pp. 263-275, March 2007.

[51] A. Koppelaar, O. Eerenberg, L. Tolhuizen, and V. Aue, "Restoration of IP-Datagrams in the DVB-H Link-layer for TV on Mobile,” Proc. IEEE ICCE, Las Vegas, USA, 2006.

[52] H. Himmanen and T. Jokela, "DVB-H Field Trials: Studying Radio Channel Characteristics," Proc. IEEE BMSB, Orlando, USA, 2007.

[53] H. Himmanen, J. Paavola, and H. Pekonen, "Improving Error Burst Tolerance at the DVB-H Link Layer," Proc. IEEE BMSB, Orlando, USA, 2007

[54] ETSI TS 102472 v1.2.1, "IP Datacast over DVB-H: Content Delivery Protocols,” Dec. 2006.

[55] A. Shokrollahi, "Raptor Codes," IEEE Trans. on Information Theory, vol. 52, no. 6, pp. 22512567, June 2006.

[56] 3GPP TS 26.346 v6.11.0, "Multimedia Broadcast/Multicast Service (MBMS); Protocols and Codecs," March 2008.

[57] ETSI TS 102591 v1.2.1, 'IP Datacast over DVB-H: Content Delivery Protocols Implementation Guidelines," April 2009.

[58] E. Stare, A. Lindberg, and M. Watson, "Simplified Model to Assess the Gain by Using Application Layer Generic Forward Error Correction (ALGFEC) for File Delivery," DVB technical document TM-CBMS 1209, Feb. 2005.

[59] L. R. Wilhelmsson, "Evaluating the Performance of Raptor Codes for DVB-H by using the Gilbert-Elliott Channel," Proc. IEEE VTC Fall, Baltimore, USA, 2007.

[60] A. Ligeti, “Single Frequency Network Planning," Ph.D. thesis, Royal Institute of Technology, Sweden, 1999.

[61] A. Ligeti and J. Zander, "Minimal Cost Coverage Planning for Single Frequency Network," IEEE Trans. on Broadcasting, vol. 45, no. 1, pp. 78-87, March 1999.

[62] R. M. Whitaker, L. Raisanen, and S. Hurley, "A Model for Conflict Resolution between Coverage and Cost in Cellular Wireless Networks,” Proc. HICSS, Hawaii, USA, 2004.

[63] H. Meunier, E. Talbi, and P. Reininger, "A Multiobjective Genetic Algorithm for Radio Network Optimization,” Proc. CEC, California, USA, 2000. 
[64] K. Deb, “Multi-Objective Optimization using Evolutionary Algorithms,” Wiley, 2001.

[65] P. Unger and T. Kürner, "Scenarios for Radio Planning of DVBH/UMTS Hybrid Networks," Proc. COST 273, TD 04-151, Duisburg, Germany, 2004.

[66] P. Unger, M. Schack, and T. Kürner, "Minimizing the Electromagnetic Exposure using Hybrid (DVB-H/UMTS) Networks," IEEE Trans. on Broadcasting, vol. 53, no. 1, pp. 418-424, March 2007.

[67] C. Heuck, "Analysis of the Performance of Hybrid (Broadcast/Mobile) Networks," Proc. IEEE ISCE, Reading, UK, 2004.

[68] C. Heuck, "Benefits and Limitations of Hybrid (Broadcast/Mobile) Networks," Proc. IST Mobile and Wireless Communication Summit, Dresden, Germany, 2005.

[69] L. Huang, K. A. Chew, and R. Tafazolli, "Network Selection for One-to-Many Services in 3GBroadcasting Cooperative Networks," Proc. IEEE VTC Spring, Stockholm, Sweden, 2005.

[70] L. Huang et al., "Efficient Group-Based Multimedia-on-Demand Service Delivery in Wireless Networks," IEEE Trans. on Broadcasting, vol. 52, no. 4, pp. 492-504, Dec. 2006.

[71] A. Bria, "Cost-based Radio Resource Management in Hybrid Cellular-Broadcasting Systems," Proc. IEEE VTC Spring, Stockholm, Sweden, 2005.

[72] A. Bria, "Future Hybrid Cellular-Broadcasting Systems for Multimedia Multicasting," Proc. IEEE ICWMC, Bucharest, Romania, 2006.

[73] T. Lohmar and U. Horn, "Hybrid Broadcast-Unicast distribution of Mobile TV over 3G Networks," Proc. IEEE LCN, Tampa, USA, 2006.

[74] T. Lohmar and U. Horn, "Evaluation of a Hybrid Broadcast-Unicast Mobile TV offering for 3G Systems," Proc. IEEE P2MNet, Tampa, USA, 2006.

[75] D. Catrein, J. Huschke, and U. Horn, "Analytic Evaluation of a Hybrid Broadcast-Unicast TV Offering," Proc. IEEE VTC Spring, Marina Bay, Singapore, 2008.

[76] M. Luby, T. Gasiba, T. Stockhammer, and M. Watson, "Reliable Multimedia Download Delivery in Cellular Broadcast Networks," IEEE Trans. on Broadcasting, vol. 52, no. 1, pp. 235-246, March 2007.

[77] T. Stockhammer, A. Shokrollahi, M. Watson, M. Luby and T. Gasiba, "Application Layer Forward Error Correction for Mobile Multimedia Broadcasting," Handbook of Mobile Broadcasting: DVB-H, DMB, ISDB-T and Media FLO, CRC Press, 2008.

[78] T. Lohmar, P. Zhaoyi, and P. Mahonen, "Performance Evaluation of a File Repair Procedure based on a Combination of MBMS and Unicast Bearers," Proc. IEEE WoWMoM, Niagara Falls, USA, 2006.

[79] D. Gómez-Barquero, D. Gozálvez, and N. Cardona, "Application Layer FEC for Mobile TV Delivery in IP Datacast over DVB-H Systems," IEEE Trans. on Broadcasting, vol. 55, no. 2, pp. 396-406, June 2009. 
[80] D. Gómez-Barquero and A. Bria, "Application Layer FEC for Improved Mobile Reception of DVB-H Streaming Services," Proc. IEEE VTC Fall, Montreal, Canada, 2006.

[81] DVB bluebook A131, “Digital Video Broadcasting (DVB); MPE-IFEC,” Nov., 2008.

[82] D. Gozálvez, D. Gómez-Barquero, and N. Cardona, "Performance Evaluation of the MPEiFEC Sliding RS Encoding for DVB-H Streaming Services," Proc. IEEE PIMRC, Cannes, France, 2008.

[83] D. Gómez-Barquero, D. Gozálvez, P. Olivas, and N. Cardona, "Multi Burst Forward Error Correction for Mobile TV Services in DVB-H Systems," submitted to IEEE Communications Magazine.

[84] P. Unger and D. Gómez-Barquero, "Coverage Gain Estimation for Multi-Burst Forward Error Correction in DVB-H Networks," Proc. IEEE BMSB, Bilbao, Spain, 2009.

[85] D. Gómez-Barquero and P. Unger, "Coverage Estimation for Multi-Burst Forward Error Correction Services in DVB-H Networks," submitted to IEEE Trans. on Broadcasting.

[86] V. Ollikainen and C. Peng, "Integration of DVB-T into DVB-H Transmission Systems," Proc. IBC, Amsterdam, The Netherlands, 2006.

[87] X. Yang, "Handover in DVB-H - Investigations and Analysis," Springer, 2008.

[88] D. Gómez-Barquero, A. Fernández-Aguilella, and N. Cardona, "Multicast Delivery of File Download Services in Evolved 3G Mobile Networks with HSDPA and MBMS," to appear in IEEE Trans. on Broadcasting.

[89] D. Gómez-Barquero and A. Bria, "Forward Error Correction for File Delivery in DVB-H," Proc. IEEE VTC Spring, Dublin, Ireland, 2007.

[90] D. Calabuig, J. F. Monserrat, D. Gómez-Barquero, and O. Lázaro, "User Bandwidth Usage - Driven HNN Neuron Excitation Method for Maximum Resource Utilization within Packet - Switched Communication Networks," IEEE Communications Letters, vol. 10, no. 11, pp. 766-768, Nov. 2006.

[91] D. Calabuig, J. F. Monserrat, D. Gómez-Barquero, and N. Cardona, "A Delay-Centric Dynamic Resource Allocation Algorithm for Wireless Communication Systems based on HNN," IEEE Trans. on Vehicular Technology, vol. 57, no. 6, pp. 3653-3665, Nov. 2008.

[92] D. Calabuig, J. F. Monserrat, D. Gómez-Barquero, and O. Lázaro, "An Efficient Dynamic Resource Allocation Algorithm for Packet-Switched Communication Networks Based on Hopfield Neural Excitation Method," to appear in Elsevier Neurocomputing.

[93] D. Gómez-Barquero, D. Calabuig, J. F. Monserrat, N. García, and J. Pérez-Romero, "Hopfield Neural Network - based Approach for Joint Dynamic Resource Allocation in Heterogeneous Wireless Networks," Proc. IEEE VTC Fall, Montreal, Canada, 2006.

[94] D. Gómez-Barquero and J. Poikonen, "Development and Applications of a DVB-H SystemLevel Simulator," submitted to IEEE Trans. on Broadcasting. 
[95] J. Poikonen, J. Paavola, and V. Ipatov, "Aggregated Renewal Markov Processes with Applications in Simulating Mobile Broadcast Systems," IEEE Trans. on Vehicular Technology, vol. 58, no. 1, pp. 21-31, Jan. 2009.

[96] J. Poikonen, "Efficient Channel Modeling Methods for Mobile Communication Systems," Ph.D. thesis, University of Turku, Finland, 2009.

[97] J. Poikonen and D. Gómez-Barquero, "Validation of a DVB-H Dynamic System Simulator using Field Measurements," Proc. IEEE BMSB, Las Vegas, USA, 2008.

[98] D. Gómez-Barquero and N. Cardona, "Fading Margin Gain due to Multi-Slot FEC in TDM Broadcast Systems," submitted to IEEE Trans. on Broadcasting.

[99] ETSI EN 300744 v1.5.1, "Digital Video Broadcasting (DVB); Framing Structure, Channel Coding and Modulation for Digital Terrestrial Television," Nov. 2004.

[100] IETF RFC 3550, “RTP: A Transport Protocol for Real-Time Applications,” July 2003.

[101] H. Fuchs and N. Färber, "Optimizing Channel Change Time in IPTV Applications," Proc. IEEE Broadband Multimedia, Las Vegas, USA, 2008.

[102] H. Himmanen, M. M. Hannuksela, T. Kurki, and J. Isoaho, "Objectives for New Error Criteria for Mobile Broadcasting of Streaming Audiovisual Services," EURASIP Journal on Advances in Signal Processing, 2008.

[103] IETF RFC 3926, “FLUTE - File Delivery over Unidirectional Transport,” Oct. 2004.

[104] M. Luby, T. Stockhammer, and M. Watson, "Application Layer FEC in IPTV Services," IEEE Communications Magazine, vol. 46, no. 5, pp. 94-101, May 2008.

[105] M. Mitzenmacher and E. Upfal, "Probability and Computing: Randomized Algorithms and Probabilistic Analysis," Cambridge University Press, 2005.

[106] IETF RFC 3450, “Asynchronous Layered Coding (ALC) Protocol Instantiation,’'Dec. 2002.

[107] IETF RFC 3451, “Layered Coding Transport (LCT) Building Block,” Dec. 2002.

[108] IETF RFC 3452, "Forward Error Correction (FEC) Building Block,” Aug. 2007.

[109] I. S. Reed and G. Solomon, "Polynomial Codes Over Certain Finite Fields," SIAM Journal on Applied Mathematics, vol. 8, no. 2, pp. 300-304, June 1960.

[110] I. S. Reed and X. Chen, "Error-Control Coding for Data Networks," Kluwer, 1999.

[111] M. Luby, "LT Codes," Proc. IEEE FOCS, Vancouver, Canada, 2002.

[112] J. Byers, M. Luby, and M. Mitzenmacher, "A Digital Fountain Approach to Asynchronous Reliable Multicast,” IEEE JSAC, vol. 20, no. 8, pp. 1528-1540, Oct. 2002.

[113] M. Mitzenmacher, "Digital Fountains: A Survey and Look Forward," Proc. IEEE ITW, San Antonio, USA, 2004.

[114] J. Byers, M. Luby, M. Mitzenmacher, and A. Rege, "A Digital Fountain Approach to Reliable Distribution of Bulk Data," Proc. ACM SIGCOMM, Vancouver, Canada, 1998. 
[115] M. Luby and A. Shokrollahi, "Mobile Data Broadcast Delivery using FEC Codes," Digital Fountain technical report, 2008.

[116] ETSI EN 301192 v1.4.2, "Digital Video Broadcasting (DVB); DVB Specification for Data Broadcasting," April, 2008.

[117] ETSI TR 102377 v1.3.1, "Digital Video Broadcasting (DVB); DVB-H Implementation Guidelines,"May 2007.

[118] M. Kalman, E. Steinbach, and B. Girod, "Adaptive Media Playout for Low-Delay Video Streaming Over Error-Prone Channels," IEEE Trans. on Circuits and Systems, vol. 14, no. 6, pp. 841-851, June 2004.

[119] H. Himmanen, T. Jokela, J. Paavola, and V. Ipatov, "Performance Analysis of the DVB-H Link Layer Forward Error Correction," Handbook of Mobile Broadcasting: DVB-H, DMB, ISDB-T and Media FLO, CRC Press, 2008.

[120] M. Gudmunson, "Correlation Model for Shadow Fading in Mobile Radio Systems," Electronic Letters, vol. 37, no. 23, pp. 2145-2146, Nov. 1991.

[121] S. R. Saunders, “Antennas and Propagation for Wireless Communication Systems," Wiley, 2003.

[122] H. Parviainen et al., "Novel Radio Channel Models for Evaluation of DVB-H Broadcast System," Proc. IEEE PIMRC, Helsinki, Finland, 2006.

[123] M. Hata, "Empirical Formula for Propagation Loss in Land Mobile Radio Services," IEEE Trans. on Vehicular Technology, vol. 39, no. 3, pp. 317-325, Aug. 1980.

[124] TeamCast and DiBcom 2008, "DVB-H Calculator - Mobile Performance Evaluator," Available online http://www.teamcast.com/

[125] D. Gómez-Barquero, A. Salieto, J. Martín, and N. Cardona, "Planificación y Despliegue de Redes de TDT Móvil DVB-H utilizando Gap-fillers," (in Spanish), Proc. Jornadas Telecom $I+D$, Valencia, Spain, 2007.

[126] K. Salehian, M. Guillet, and B. Caron, "On-Channel Repeater for Digital Television Broadcasting Service," IEEE Trans. on Broadcasting, vol. 48, no. 2, pp. 97-102. Jun 2002

[127] K. Salehian, B. Caron, and M. Guillet, "Using On-Channel Repeater to Improve Reception in DTV Broadcasting Service Area," IEEE Trans. on Broadcasting, vol. 49, no. 3, pp. 309-313, Sept. 2003.

[128] IST-PLUTO project report, "Initial Report on Echo Measurement," July 2006. http://dea.brunel.ac.uk/pluto/

[129] FURIA project report, "SFN Networks - Gap-Fillers," (in Spanish) March 2007. http://www.furiapse.com/

[130] K. Beeke, "Self-Interference in SFNs," EBU Technical Review, July 2007. 
[131] K. Deb, A. Pratap, S. Agrawal, and T. Meyarivan, "A fast and elitist multi-objective genetic algorithm: NSGA-II," IEEE Trans. on Evolutionary Computation, vol. 6, no. 2, pp. 182-197, 2002.

[132] E. Zitzler, M. Laumanns, and L. Thiele, "SPEA2: Improving the strength Pareto evolutionary algorithm for multiobjective optimization," Evolutionar Methods for Design, Optimization, and Control, pp. 95-100, 2002.

[133] D. Krajzewicz, M. Bonert, and P. Wagner, “The Open Source Traffic Simulation Package SUMO,” Proc. RoboCup, Bremen, Germany, 2006.

[134] T. Kürner, "Digital Mobile Radio towards Future Generation Systems: Propagation Models for Macro-Cells," COST Action 231 Final Report, pp. 134-148, Brussels, Belgium, 1999.

[135] P. I. Bratanov and E. Bonek, "Mobility Model of Vehicle-Borne Terminals in Urban Cellular Systems," IEEE Trans. on Vehicular Technology, vol. 52, no 4, pp. 947-952, July 2003.

[136] 3GPP TR 25.993 v6.15.0, "Typical Examples of Radio Access Bearers (RABs) and Radio Bearers (RBs) supported by Universal Terrestrial Radio Access (UTRA),” Oct. 2007.

[137] 3GPP TR 25.803 v6.0.0, “S-CCPCH Performance for MBMS," Sept. 2005.

[138] Celtic Wing TV project, http://projects.celtic-initiative.org/WING-TV/

[139] ETSI EN 302304 v1.1.1, "Digital Video Broadcasting (DVB); Transmission System for Handheld Terminals (DVB-H),” Nov. 2004.

[140] R. Brugger and D. Hemingway, "OFDM receivers - Impact on Coverage of Inter-Symbol Interference and FFT Window Positioning,” EBU Technical Review, July 2003.

[141] D. Milanesio et al., "Wing TV Network Issues," Celtic Wing TV project report, May 2006.

[142] M. Failli, “Digital Land Mobile Radio Communications," COST Action 207 Final Report, pp. 135-147, Luxemburg, Luxemburg, 1989.

[143] R. Parviainen et al., "The Validation of the Novel DVB-H Radio Channel Models," Proc. IEEE Chinacom, Shangai, China, 2007.

[144] A. Ligeti, "Coverage Probability Estimation in Single Frequency Networks in Presence of Correlated Useful and Interfering Components," Proc. IEEE VTC Fall, Amsterdam, The Netherlands, 1999.

[145] K. Beeke, "Spectrum Planning - Analysis of Methods for the Summation of Log-normal Distributions," EBU Technical Review, October 2007.

[146] K. L. Baum, T. A. Kostas, P. J. Sartori, and B. K. Classon, "Performance Characteristics of Cellular Systems with Different Link Adaptation Strategies," IEEE Trans. on Vehicular Technology, vol. 52, no. 6, pp. 1497-1507, Nov. 2003.

[147] X. Qiu and K. Chawla, "On the Performance of Adaptive Modulation in Cellular Systems," IEEE Trans. on Communications, vol 47., no. 6, pp. 884-895, June 1999. 
[148] E. N. Gilbert, “Capacity of a Burst-Noise Channel,” Bell System Technical Journal, vol. 39, pp. 1253-1265, Sept. 1960.

[149] E. O. Elliott, "Estimates of Error Rates for Codes on Burst-Noise Channels," Bell System Technical Journal, vol. 42, pp. 1977.1997, Sept. 1963.

[150] DVB bluebook A122, "Frame Structure Channel Coding and Modulation for a Second Generation Digital Terrestrial Television Broadcasting System (DVB-T2)), June 2008.

[151] C. Hellge, S. Mirta, T. Schierl, and T. Wiegand, "Mobile TV with SVC and Hierarchical Modulation for DVB-H Broadcast Services,” Proc. IEEE BMSB, Bilbao, Spain, 2009. 

